\title{
The alternating-direction implicit namerical solution of the time-dependent, three-dimensional, single fluid, resistive magnetohydrodynamic equations
}

\author{
Charles H. Finan !!! \\ (Ph.D. Thesis) \\ December 1980
}

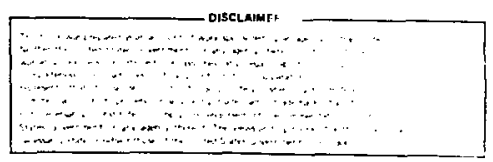

LAWRENCE LIVERMORE LABORATORY University of California - Livermore. California 945.30 
The Alternating Direction Inplicit Numerical Solution of the Time-Dependent, Three-Dimensional, Resistive, Single Fluid Magnetohydrodynamic Equations

\section{By}

\section{CHARLES HENRY FINAN III}

B.S. (Long Island Untversity, New York) 1971

M.S. (University of Calffornia, Davis) 1973

\section{DISSERTATION}

Submitted in partial satisfaction of the requirements for the degree of

\section{DOCTOR OF PHLOSOPHY}

in

Englneering-Applied Science

in the

\section{GRADUATE DIVISION}

of the

\section{UNIVERSITY OF CALIFORNIA}

\section{DAVIS}

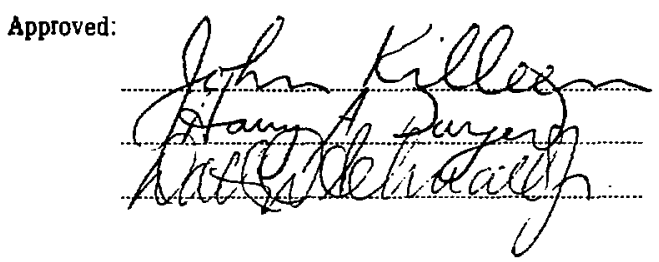

Committee in Charge

Deposited in the University Library 


\title{
THE ALTERNATING-DIRECTION IMPLICIT NUMERICAL SOLUTION OF THE TIME-DEPENDENT, THREE-DIMENSIONAL, SINGLE FLUID, RESISTIVE MAGNETOHYDRODYNAMIC EQUATIONS
}

by

Charles H. Finan III

\author{
Department of Applied Science \\ University of California, Davis \\ Livermore, California 94550 \\ June, 1980
}

ABSTRACT

Resistive magnetohydrodynamics (MHD) is described by a set of eight coupled, nonlinear, three-dimensional, time-dependent, purtial differential equations. A computer cofe, IMP (Implicit MHD Program), has been developed to solve these equations numerically by the method o. finite differences on an Eulerian mesh. In this model, the equations are expressed in orthogonal curvilinear coordinates, making the cude applicable to a variety of coordinate systems. The Douglas-Gunn algorithm for Alternating-Direction Implicit (ADI) temporal advancement is used to avoid the limitations in timestep size imposed by explicit methods. The equatious ares lved simultaneously to avoid syncronization errors. While the continuity and magnetic flux equations are expressed as conservation laws, the momentum and energy equations are nonconservative. This is to: (1) provide enhanced numerical stability by eliminating errors iutroduced by the nonvanishing of $\nabla \cdot B$ on the finite difference mesh; and, (2) allow the simulation of low 6 
plasmas. 'lo allow fe general simulations, the boundary conditions may be Dirichlet, Neumann, or perlodic. A conservation boundary condition based on the physical properties of the wall is presented. The resulting finite difference equations are a coupled system of nonlinear algebraic equations which are solved by the Newton-Raphson 1teration technique.

We apply our model to a number of problems of importance in magnetic fusion research. Ideal and resistive iuternal kink instabilities are simulated in a Cartesian geometry. Growth rates and nonlinear saturation amplitudes are found to be in agreement with previous analytic and numerical predictions. We also simulate these instabilities in a square cross section torus. This demonstrates the versatility of the orthogonal curvilinear coordinate representation employed by our model. In both geometries, long time simulations in resistive plasmas display final states with finite flow fields. Finally, we perform a simulation of the spontaneous field reversal in a high current pinch. In this case, relaxation to a fleld reversed grometry is demonstrated both for a plasma that initlally has a high pinch ratio $\left(L_{G} / \mathrm{B}_{2}>1.6\right)$, and for one that initially has a low pinch ratio and is subjected to an external electric field to increase the internal current. In these final Reversed Field Pinch (REP) cases, we find that the resistive internal kink is the mechanism which causes the field reversal. 
To my sons

Chris

and

Mike 
TABLE OF CONTENTS

Page

I Introduction $\ldots \ldots \ldots \ldots \ldots \ldots \ldots \ldots \ldots \ldots \ldots \ldots \ldots \ldots \ldots \ldots$

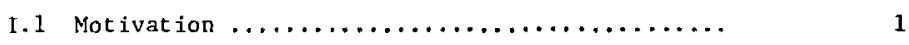

1.2 Fusion Energy Sources $\ldots \ldots \ldots \ldots \ldots \ldots \ldots \ldots \ldots$

I. 3 Plasma Instabilities ..................

I.4 Computational Concepts ................ 9

1.5 Previous Work ..................... 11

I.5.1 Two-Dimensional Codes ............ 12

I.5.2 Three-Dimensional Codes .......... 14

I. 6 scope of Present Work ................. 16

I1. Mathematical Model ...................... 26

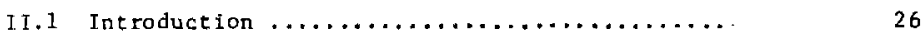

I 1.2 Resistive Single Fluid MHD Equations ......... 26

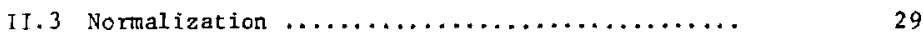

II.4 An Alternate Mode1 .................. 31

II.5 forthogonal Curvilinear Soordinates ..........

III. Computationai Techniques ................. $4 z$

III.1 Introduction $\ldots \ldots \ldots \ldots \ldots \ldots \ldots \ldots \ldots \ldots \ldots \ldots \ldots$

III.1.1 Basic Numerical Analysis .......... 48

III. 2 The Computational Mesh ............... 55

III.3 Spatial Finite Differencing ............... 59

III. 3.1 Flux Term Differencing ............ 59

III.3.2 Force Term Differencing ........... 62

III. 4 Temporal Finite Differencing ............. 63

III.5 Linearization of the Finite Difference
Equations $\ldots \ldots \ldots \ldots \ldots \ldots \ldots \ldots \ldots \ldots \ldots \ldots \ldots \ldots \ldots$

III. 6 Solution of Difference Equations ..........

III. 7 Boundary Conditions ................. 80

III.7.1 GHOST Point Boundary Conditions ...... BI

III.7.2 Flux Surface Boundary Ccaditions ..... 90 
III. 8 Miscellaneous Comput.ational Problems ........ 91

III.8.1 Advection Terms ............... 91

III.8.2 Iteration Controls ............. 92

III.8.3 Fizestep Controls ............. 92

IV. The Intemal Kink in Cartesian Geometry .......... 95

IV.1 Introduction ..................... 95

IV. 2 Equilibrium $\ldots \ldots \ldots \ldots \ldots \ldots \ldots \ldots \ldots \ldots \ldots \ldots$

IV. 3 Initial Conditions ................... 98

IV.4 Boundary Conditions and Normalization ........ 100

IV.5 Diagnostics $\ldots \ldots \ldots \ldots \ldots \ldots \ldots \ldots \ldots \ldots \ldots \ldots \ldots \ldots$

IV. $s$ Results ........................ 103

IV.6.1 Velocity Field Perturbation, Ideal MHD ..................... 10 ?

IV.6.2 Perturbation by Linear Eigenmodes, Ideal MHD ................... 104

IV. 6.3 Resistive Plasma Long Time Simulation ................... 105

IV.6.4 Resistive P1asma Dependence of Growth Rate on 5 ................... lno

IV. 6.5 Resistive Plasma Without Bolancing Electron Field and Cooling Terms .... L

IV.7 Summary $\ldots \ldots \ldots \ldots \ldots \ldots \ldots \ldots \ldots \ldots \ldots \ldots \ldots \ldots$

V. The Internal Kink Tn a Square Cross Section Torus ....

V.I Introduction $\ldots \ldots \ldots \ldots \ldots \ldots \ldots \ldots \ldots \ldots \ldots \ldots \ldots$

V.2 Equilibrium and Initial Conditions ......... $1+h$

V.3 Boundary Conditions and Normalization ....... 1,7

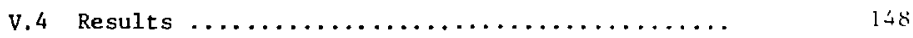

V.4.1 Equilibrium Test .............. 148

V.4.2 $\mathrm{n}=1, \mathrm{n}=4$ Ideal Kink Mode ....... 149

V.4.3 $\mathrm{m}=1, \mathrm{n}=2$ IdeaI Kink Mode ....... 150

V.4.4 Long Time Resistive Simulation ...... 151

V.5 Summary ...................... 152 
VI. Spontaneous Reversed Field Pinch ................. 179

VI.1 Introduction ...................... I79

VI.2 Initial Conditions ................... 180

VI. 3 Boundary Conditione and Zoning ........... 181

VI.3.1 Case $1 \ldots \ldots \ldots \ldots \ldots \ldots \ldots \ldots \ldots \ldots 18 \ldots \ldots . \ldots 1$

VI.3.2 Case 2 and Case $3 \ldots \ldots \ldots \ldots \ldots \ldots \ldots$

VI.4 Artifictal Viscosity .................. 183

VI.5 Results .......................... 184

VI.5.1 Case 1: High Initial $x \ldots \ldots \ldots \ldots \ldots .184$

UI.5.2 Case 2: Externally Applled Electric Field, $q_{0}=.8 \ldots \ldots \ldots \ldots \ldots \ldots \ldots \ldots$

VI. 5.3 Case 3: Externally Applied Electric Field, $q_{0}=1.1 \ldots \ldots \ldots \ldots \ldots \ldots \ldots \ldots$

VI.6 Summary $\ldots \ldots \ldots \ldots \ldots \ldots \ldots \ldots \ldots \ldots \ldots \ldots \ldots \ldots \ldots$

II. Summary and Conclusions ..................... 209

VII.1 Sumary .......................... 209

VII. 2 Conclusions $\ldots \ldots \ldots \ldots \ldots \ldots \ldots \ldots \ldots \ldots \ldots \ldots \ldots \ldots$

VII.3 Future Work $\ldots \ldots \ldots \ldots \ldots \ldots \ldots \ldots \ldots \ldots \ldots \ldots \ldots$

ACKNOWLEDGMENTS $\ldots \ldots \ldots \ldots \ldots \ldots \ldots \ldots \ldots \ldots \ldots \ldots \ldots \ldots \ldots$

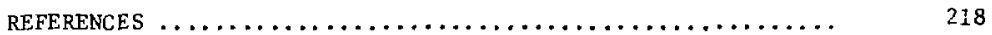

APPENDIX A. 2-D Tearing Mode Simulation ............. 225

APPENDIX B. The REDUCE Generator Code $\ldots \ldots \ldots \ldots \ldots \ldots \ldots \ldots . . \ldots 232$ 


\section{CHAPTER I}

- INTRODUCTION

\section{I.1 Motivation}

The world's need for rellable, long term sources of energy has become more obvious as the supply of fossil fuels has decreased. In order to proride these energy sources, research has begun in a number of areas. old enercy sources are being re-examined in the light of modern technology, including such diverse areas as the use or wind power for electrical generation and coupling MHD (magnetohydrodynamic) generators to coal-fired electrical generators to raise their efficiency. In addition, new energy sources such as solar, geothermal and controlled nuclear fusion are being explored.

Of these new sources, controlled fusion energy has a number of attractive features. First, the fuel for fusian. rlants, deuterium, is found in ordinary water (.0153\% of the hydrogen in sea water is deuterium ${ }^{1}$ ), making it a viable source of energy which could supply the world's enerğ needs for millions of years. Secondly, flel costs would be a negligiole part of the total plant construction and operating budgets. Finally, compared to the present day nuclear fission power flants, the fusion nower plant poses few significant environmental cr safety hazards.

In order to achieve a working thermonuclear fusion reactor, the deuterium fuel must be ionized and contained by magnetic fields at a high kinetic temperature (hotter than the sun's core) for a sufficient amount cf $t$ tne to allow the thermonuclear reactions to produce more power than was expended in the conflnement and heating process. The greatest obstacle to 
the production of these confined, high temperature plaswas (an lonized gas) Is the onset of large-scale Instabilities. These instabilities are described by the magnetohydrodynanc (MHD) equations which mode1 the plaswa as a conducting fluid immersed in a magnetic field.

The first approach to understanding these large scale instabilicles was accomplished by studying the effect of small perturbations in the linearized MHD equations. If the small perturbations grew in time in a given magnetic field configuration, then that magnetic field configuration was declaced unstable. While only the most idealized situations could be treated analytically, the gross MHP Instabflities became better understood and, consequently, more avoldable.

More recently, researchers have come to realize that some instabilitfes can he tolerated, and in fact can be beneffatal, provided they do not disrupt the plasma before a sufficlent amount of power has been produced. Consequent1y, there is great interest in the nonlinear development of MHD instabilities and the final state of plasmas Influenced by these instabilities. The analytical solution of the MED equations (a system of 8 coupled, nonlinear partial differential equations) is, in ganeral, impossible. Thus, numerical calculations are required for the investigations.

The power of modern computers has barely kept pace with requirements of the Fusion comnunity. In the late 1950's and early 1960's, computations were limited to one-dimensional models. In the middle and late $1960^{\prime} s$ and early 1970's, two-dimensional effects were being studied. TwL-timensional 
MHD codes, both 1 fnear and nonlinear, are now commonplace at al! major fuston rese: th facilities. Currently, models are being developed to study nonlinear effecte in thret dimerisions. These codes tax the capabilit les of even tile fastert computes, the so-called class VI machines, whiri ran perform up to 250 million floating point ontations per second. Consequently, it is of the utaost importance to design a si iution algorlihm that efficiently utilizes the avallable resourc.'s.

This thesis details the developtnent of a computst rode IMP (implicit MHD Program). In IMP, the full set of resistive AtD equations, rithout any ordering of terms, are solved in three dimenstons by the methot of finfte differences. The equatiouis are expressed in orthogonal curvilinear cwo:d:nates to allow simulations in varying geometrles. Implicit time advincement techniques are used to avold numertsal limftations on the $t$ imestep sizo, The code has been developed on a CDC-7600 computer, whi:h is approximat, :y four times slower than the aforementioned Class VI machine, and has ty.ical running tirses of 1 to 25 how. 3 , depending on the problem.

\section{2 Fusion Energy Sources}

As in any other jower plant, for the fusion reacto- to be feasible, it must produce rore power than is necessary to initiate and sustain its reaction. This may be expressed as the rat to

$$
Q \equiv \frac{\text { fusion power out }}{\text { total power in }}
$$

where $Q$ is called the efficiency of the reactor and must be greatcr than unity. This requt rement can be calculated based on a deuterium-tritium 
(D-T) gas heated to a kinetic temperature of $10 \mathrm{keV}\left(=1.1605 \times 10^{8}{ }^{\circ} \mathrm{K}\right)$. At this temperature, the gas is completely ionized and 1 s called a plasma. The fusion power out can be determined from the known cross sections for fusion and the eriergy released per fuston event. The total power in inc ${ }^{1}$ udes the energy reeded to heat the $D-T$ gas and to confine it long enough to react. Based on these considerations, the requirement $Q>1$ becomes (the Lawson criterion ${ }^{2}$ ):

$$
\text { nт }>10^{14} \mathrm{sec} / \mathrm{cm}^{3}
$$

where $\mathrm{n}$ is the particle number density and $T$ is the confinement time.

There are currently two approaches being taken to satisfy the Jawson criterion. The first method is to implode a deuterium pellet using powerful laser or ion beams, thereby producing a dense plasma (n $i 10^{25} \mathrm{~cm}^{-3}$ ). In this case, the confinement time is extremely short $\left(\tau \approx 10^{-11} \mathrm{sec}\right)$. The second method involves confining a lower density plasma $\left(n \approx 10^{15} \mathrm{~cm}^{-3}\right.$ ) for a sustained period of time $\left(\tau r_{n} .1 \mathrm{sec}\right)$. The plasma cannot be confined by material walls, as they would provide too great an energy loss mechanisa. At thermonuclear densities and temperatures, tine mean free path for collisions between particles of the plasma is many times any reasonablysized contalnment vessel. Therefore, the collisions of hot particles with the wall would result in a transfer of energy to the wall. The cooled particle would then return to the reacting region and further lower the temperature of the remaining particles. The collisions could also cause sputtering of wall materials into the plasma. These contaminants would 
require energy to reach the plasma temperature and would then increase energy losses due to bremsstrahlung radiation. 3

Materlal walls do not solve the confinement problem. However, since the $\mathrm{T}-\mathrm{T}$ gas is fully tonized, magnetic fields car be substituted. One basic effect can be found in many of the magnetic fielc geonetries that have been trled to date. A current In the plasma produces a magnetic field whici "pinches" the plasma, Consıder the cylinder of plasma shown in Fig. I.l. If a current is driven along the length of the plasma (In the $z$ direction), a magnetic field will be induced in the $\theta$ direction. The $B_{\theta}$ field exerts a pressure $\mathrm{B}_{\theta}^{2} / 8 \pi$ ( $1 \mathrm{n}$ cgs units) which compresses or pinches the plasma columl. As the colum contracts the magnetic pressure facreases ( $\left.P \propto B_{A}^{2} \times 1 / r^{2}\right)$. Thus, the pinching continues unt1l the internal pressure ot the constructed plasma cotuca has risen to equal the magnetlc pressure.

\section{3 Plasma Instabilities}

The major problem with finched discharges and, for that matter, with any system in which charged particles are confined bv magnetic fields, is the exfstence of large scale instabllties. The major instabilities are known as the sausage or necking-off mode, the kink or wriggling mode a.nd the flute modes. All of these Instabilities will grow rapidly enough to destroy the plasma confinement well before the Lawson criterion can be satisfled. The driving mechanism for these instabilities can be spen from simple phystcal arguments. It is assumed in the following discussion that there is no magnetic field in the plasma; that the current flows only on the surface of the plasma; and that the plasma cylinder is of infinite length. 
The sausage mode occurs when there is an azimuthally symmetric localized constriction of the plama (see Fig. I.2). Since the current is a constant along the discharge, th: local magnetic pressure is increased at the constrictica $\left(P_{M} \approx 1 / r^{2}\right)$. Hence, this reglon then tends to become more and more constricted thereby forcing the plasma into the nonconstricted areas causing them to expand. Eventually, the plasma breaks up into individual "links", destroylng the current path and the discharge.

The kink or wriggling instabllity (Fig. I.3) accurs when a hend forms in the plastia column. In this case, the magnetic pressure is increased nn the concave side of the plasma and decreased on the convex side. Thus, the magnetic forces are $n$. ng to increase the original perturbation. In this case, the plasma continues to kink or wriggle until it collides with the walls of the contalnment vessel.

The flute instability (FIg. I.4) is analogous to the class of interchange instabilfties found in normal hydrodynamles. In this case, the plasma is able to sip through the magnetlc field lines without distorting them--and interchange with them--much as in the case of a heavy fluid supported by a light one (the Rayleigh-Taylor instability).

The sausage mode can be stabilized by including an embeddec longitudinal magnetic field $\left(B_{z}\right)$. Unlike the plasma, this field cannot be forced out of the constricted region. When the plasma cross section is decreased, the internal magnetic pressure $\left(\propto \mathrm{B}_{\mathrm{z}}^{2}\right)$ 1ncreases, offsetting the increased external magnetic pressure. The embedded field also acts to stabilize short wavelength kink modes since the fleld 1 ines exhibit a cension which is 
similar to that of a rubber band being stretched. The magnetic tension along the plasma column opposes the magne ic pressure pushing the colum, thus intibiting growth of the mode. The tension is not, hawever, very effective in opposing long wavelength modes. These modes are controlled by surrounding the plasma with a conducting shell. Image currents in the shell form magnetic fields (via a Lenz's Law effect) which oppose the kinking of the plasma. The flute modes are stabilized by magnetic shear, that is, by having the fleld lines change their pitch $\left(\alpha \mathrm{B}_{3} / \mathrm{B}_{\theta}\right)$ as a function of position. In general, the magnetic fields in the cylindrical pinch have shear since the magnetic fleld is a function of radius. The magnetic shear makes the interchange modes energetical1y unfavorable as they now must do work to deform the magnetic field.

Pinch experiments do not, of course, have infinite length. Most pinch experiments are now being performed in toroidal devices. The straight cylinder can be used to analytically approximate a torus. The major radius $R$ of the torus now fixes the length of the cylinder to be $2 \pi R$. The cylinder also must have periodic boundary conditions. These conditions limit the range of wavelent hs of modes that can exist in the system. Only integral numbers $n$ can exist in the $z$ or toroidal direction. The same argument holds for the poloidal direction (the short way around the torus). Since the poloidal coordinate $\theta$ is periodic, only modes of integral number m can exist. A wave number $\underline{k}, \underline{k} \equiv k_{\theta} \hat{\theta}+k_{z} \hat{z}$ where $\hat{\theta}$ and $\hat{z}$ are in the $\theta$ and $z$ directions can be written such that

$$
k_{\theta}=1 / \hbar_{\theta}=2 \pi / \lambda_{\theta}
$$


and

$$
k_{7} \quad i / \lambda_{z}=2 \tau / \lambda_{z},
$$

where $\lambda_{g}$ and $\lambda_{z}$ are the wavelengths in the $\theta$ and $z$ directions. At a given miner radius $r$

$$
\lambda_{0}=2 \pi r / m
$$

and for major radius $R$

$$
z_{z}=2 \pi k / n
$$

where: $m$ and $n$ are the poloidal and toroldal mode numbers. This yields

$$
k=m / r \dot{\theta}+n / R \hat{z} .
$$

The plasma tends to be unstable when the perturbation aligns itself with the magnetic field since in this configuration the instability is required to do a minimum of work on the field. This condition can be stated as $\underline{k} \cdot \underline{B}=0$ (the wavefronts are perpendicular to the wavevector). For a give: magnetic field, $\underline{B}=B_{\theta} \hat{\theta}+B_{z} \hat{z}$, we have

$$
\frac{r B_{z}}{R B_{\theta}}=-\frac{m}{\pi} .
$$

At any point where the ratio of the magnetic fields reduces to a rational 
number, an instability can exist. The ratio $\frac{{ }^{B} z}{\mathrm{RB}_{\theta}}$ is called the safety factor $q$ and surfaces where $q$ is rational are called mode-rational or singular surfaces.

The modes described in this section are also named for their poloidal mode number. The sausage mode results from an $m=0$ percurbation, the kink Erom an $m=1$ and the flute modes from $m \geq 2$. The $m=1 \mathrm{kink}$ mode is the only mode in which there is a gross motion of the plasma column. The ratio of the poloidal to toroidal mode numbars describes the helfcal pitch of mode.

\section{I.4 Computational Concepts}

In order to represent continuous functions to tho computer, the tuncinn values are defined on a discrete set of points in space called a mesh. The functions are also evaluated at discrete points in time called levels. The differential equation is then replaced by a finite filference equation which must have the properties of consis'ency, accuracy, stability and efficiency. A finite difference approximation is consistent if, in the limit of small time and space steps, the difference equation becomes the differential equation. The accuracy of the finite difference approximation is degraded by truncation errors and by round-off errors (caused by inite precision arithmetic on the computer). A numerical mechod is stable if a sinall error at any stage in the calculation produces a smaller rotal error. The efficiency of the finite difference algorithm can be determined by its use of the available resources weighted by the difficulcy of the problem to be solved (efficiency tends to be a highly subjective topic). A finite difference mesh is call Lagrangian whon it moves with the fluid. It is 
called Eulertan when it is stationary in the laboratory frame. If the mesh has some arbitrary veloctty in the laboratory frame, it is called a generalized mesh. Th: equations are advanced in time via elther explicit or implicit techniques. An explicit method uses only information known at preceding time levels io advance to the new time level. An implicit method couples information at the new time level in the advancement algorithm. The explicit techniques, whlle very fast per timestep, have a 1 imitation as to the size of the timestep that can be used. This limit, which is called the Courant condition, is based on information transmittal rates in the finite difference mesh. Information rates in the MHD equations scale with the Alfven velocity which is proportional to the magnetic field lntensity and inversely proportional to the square root of the plasma density. This ratio leads tc ver: large velocitles in areas of little plassa because where the magnetic ffeld is strongest, the plasma density is smallect. The implicit techniques have the timestep size limitation reizxed (totally removed for linear finte difference equations with colstant coefficients). but are considerably more complex to formulate and are more cost 1 y to calculate per timestep as the solution of a system of coupled equations must be solved ( 1 for each mesh point). For multidimensional problems, the coupled system of equations becomes intractable. In order to avoid this limitation, a rechnique known as the Alternating Direction Implictt (ADI) methed has been devistd. ${ }^{4}$ In two dimensions this algorithm breaks the time advancement into two steps. In the first step, variables are advanced Implicitly in one dimension and explicitly in the other dimension to a half time level. In the second step, the procedure is reversed and the direction 
that was advanced implicitly is advanced expllcitly and vice versa. The obvious generalization of this procedure to three dimensions by using three advances of one-third time level each is not numerically unconditionally stable. A stab1o generalization 15 given in a paper by Douglas and Gunn. 5

The details of the various schemes to be named in the next section may be found in such works as Richtmyer and Morton ${ }^{6}$ or Potter. ${ }^{7}$

\section{5 Previous Work}

The numerical study of MHD stability has followed two paths. In the first, the linearized MHD equations are treated as an initial value problem. 8,9 The equilibrium state of the system is specifled and is given a perturbation. The MHD equations are integrated forward in iime to trace the evolution of the plasma. The fastest growing instabiiity eventually dominates over all other moticns. The second approach which has been used very successfully is to utilize the energy principle ${ }^{10-15}$ to gain information sn the full spectrum of ideal MHD instabilities. After the limsar modes have been determined by either of the atove methods, the resultant eigenmodes may be used to start a nonlinear calculation which can then follow their growth to some final state. 16,17

The nonlinear codes are net limfted to stability studies. They are also used to simulate total experiments. By incorporating cime dependent boundary conditions, such as circuit equations, a simulation can start with a neutral gas, and trace its ionization (via a Saha equation of state ${ }^{18}$ ), and pinching. In this section, the development of two- and threedimensional inttlal value codes will be reviewed. 


\section{I.5.1 Two-Dimensional Codes}

Most two-dimensional ccdes employ an Eulerian mesh as in the codes of Roberts et al. 19 Roberts and Weiss, ${ }^{20}$ Weiss, ${ }^{21}$ Duchs, ${ }^{22}$ Freeman and wane, ${ }^{23}$ and Peacock et al. ${ }^{24,25}$ A11 the aformentioned codes use explicit finite difference techniques. One of the difficulties encountered when using a Eulerian mesh is the accurate treatment of advective derivatives (term of the form $\underline{v} \cdot \nabla$ ). Roberts and Welss use a fourth order finite difference approximation of the advection terms. Freeman and Lane use an explicit, Lax-Wendroff algorithm with a Lapidus $^{26}$ artificial diffusion term added to each equation to give added stability. The Peacock code uses a two fluid model with a LaxWendroff scheme. The Roberts, Roberts and Welss, Freeman and Lane, and Peacock codes all examine the $(r, z)$ plane. Duchs has considered the $(r, \theta)$ plane.

Explicit linearized codes have been written by Sykes dná Wesson, 27 and Bateman et al. ${ }^{9}$ These have been used to study stability in rectangular and toroidal geometries. The Sykes and Wessen code uses the iax-Wendroff scheme while Baceman et al. employ a leapfrog algorithm. These codes produce IInear eigenmodes that can be used as initlal nerturbations in tt.e nonlinear codes.

The only recent Lagrangian MHD calculation in two dfmensions is the code of White et al. ${ }^{28}$ In this code, the set of equations are reduced by applying the so-called Tokamak ordering $\left(\mathrm{B}_{z}>\mathrm{B}_{\theta}\right)$. The torus is approximated as a cylinder and helical symmetry is imposed on 
the system. Thus, the $(r, \Phi)$ plane is examined where

$$
\Phi \equiv m \theta+\mathrm{kz} \text {. }
$$

The plasma is considered to be incompressible. With these constraints, the magnetic field and velocity are computed from potential functions. White at a1. ${ }^{29}$ have also developed an Eulerian code based on the reduced equation that has been used to study the growth of magnetic islands in a resistive plasma.

Morse $^{30}$ has used the Particle-in-Cel1 (PIC) method to perform fluid simulations of plasma guns. The fluid PIC model divides the computational region into a set of regular Eulerian zones for computing field variable (i.e., pressure or fluid velocity). Material is transported from cell to cell by using discrete particles. Thus mass is mored in a Lagrangian manner.

Hertwech and Schneider, ${ }^{31}$ Anderson, 32 and Jardin ${ }^{33}$ use generalized mesh techniques. In these codes, flux coordinaces are used whirh allow for better computation of thermal conduction. Hertwech, Schneider, and Jardin use a nonorthogonal mesh while Anderson keeps an orthogonal mesh and rezones after each timestep. Jardin's code employes a mixed explicit-implicit time advancement algorithm, while Anderson uses a timestep splitting implicit method. The HertwechSchnetder code is explicit. 
Lindemuth and Killeer ${ }^{34}$ introduced the first ADI code in 1970 in which all terms in the two fluid MHD equations, Including transport terms, are differenced implicitly. Lindemuth has since spectalized the code to include only one cumponent of the magnetic field and two velocity components. ${ }^{35}$ This new code has successfully simulated zpinch implosions. Lui and $\mathrm{Chu}^{36,37}$ use implicit methods to simulate screw and belt pinches; however, in their code, the transport coefficients are calculated by using an explicit predictor step. Schnack ${ }^{16,17,38}$ introduced an ADT code to solve the full set of single fluid resistive MHD equations. This code has been used to extensively study resistive tearing Instabilities in a variety of coordinate systems. Reviews of other one- siid cwo-dimensional work may be found in Roberts and Potter, ${ }^{25}$ Killeen, ${ }^{39}$ and Brackoill. 40

\section{I.5.2 Three-Dimensiona? Codes}

Currently, three-dimensional calculations are the subject of active research. Wooten et a1. ${ }^{41}$ have generalized the two-dimensional Iinear code of Bateman to solve the nonlinear ideal MHD equations in three-dimensions. This code uses an Euletian mesh with the same explicit, leapfrog time advancement algorithm as is found in the twodimenstonal code and has been applied to the simulation of nonlinear ideal MHD internal kink Instabllities. Cochran ${ }^{42}$ and Pritchet ${ }^{43}$ have developed explicit, Eulerian codes which include constant diffusion coefficlents and also utilize leapfrog algorithm. They too are applied to study the nonlinear development of internal k1nk instabilities. Pritchett has also demonstrated spontaneous field reversal caused by an internal kink mode in a highly pinched plasma. 
Sykes and Wesson ${ }^{44,45}$ have extended their two-dimensional code to three dimensions. I'hey include tensor resistivily, thermal conductivity, and a radiation loss meihanism. This code uses a generalized Lax-Wendroff difference approximation on an Eulerian sesh and has successfully simu?ated the "sawtooth" $4 \dot{6}$ instabillty observed in Tokamaks. This instability is characterized by a "sawtooth" or relaxation oscillation in the soft $x$-ray emissior. It has also simulated the spontaneous field reversal caused by $m=1$ kink instabilities that has veen observed in the Culham Reversed Field Pinch ${ }^{47}$ experiment.

Brackbil1 ${ }^{40}$ has developed an ideal MHD code which utilized a generalized (seri-Lagrangian) mesh based on the ICED-ALE techrique of Hirr, Cook, and Am;den. 48 The code uses an implicil Lagrangian time advance followed by a rezone phase designed to reduce distortion of the mesh and has been applied to the study of the 6 pirch and the internal kink instability. Currently, Brackbil1 49 is reporting on a refinement to his code based on the work of Jardin which identifies the fast timescale terms. Only these terms have been differenced fully implicitly. This selective or semi-1mplicit technique is twice as fast as the older code.

Strauss ${ }^{50}$ has extended the reduced model of white et al. ${ }^{28}$ to three-dimensions. He uses an Eulerian mesi, with a leapfrog time advancement algorithm. As in many of the leapfrog based codes, diffuston terms were differenced via the Dufort-Frankel method. Strauss has applied his code to the nonlinear evolution of the internal 
kink mode in the low $B$ ( $B$ is the ratio of plasma energy to confining magneric field energy), large aspect raflo (racio of length to cross section), incompressible limft in a rectangular pipe.

Jardin and Johnson 51 have extended Jardin's "dynamical grid method" to three dimensions. In this work, the "Tokamak ordering" is used to reduce three three-fimensional implictt algorithm to a series of two-dimensional impiicit (ADI) solutions coupled by un explicit solution in the third direction and has been applied to sonlinear studies of internal instabilities in Tokamaks.

Hicks et al., 52 have reported on a series oi codes which investigate the nonlinear resistive evoluíion of plasmas in Tokamaks. Once again, the ascumptions of Tokamak ordering and low $B$ are made. The code uses an Eu?erian grid and a mixed implicit (ADI) and explicii time advancement algorithm. Another code Eased on a Fourier transform technique has also been reported by Hicks in ihis paper.

\section{6 Scope of Present Work}

The three-dimensional calculations discussed in the previous section have limited applications. A number of them treat only ine ideal MHD approximatior or, if they have included non-ideal effects such as finite resistivity, are 1 imited in achievable timestep by the use of explicjt finite difference algorithms. A number of the codes use Tokamak ordering to remove fast timescales from the proilem. These codes are Iimited to low B devizes. 
In this work, a computer program, ISP (Implicit MlHD Program), which does not suffer these limitations is described. In IMP, the full set of resistive MHD equations is solved by implictt teciniques on a variable Eulerfan mesh. The equations are finite differenced in orthogonal curvilinear coordinates to allcw the spe ification of arbitrary coordinate representations. The evolution of the pressure, rather than the total energy density, is followed in time to allow the simulation of both htgh and low $F$ plasmas. The model used in IMP is given in Chapter II. The use of the Douglas-Gunn alporithm (DGA) for alcernating direztion implicit timesteps allows the use of a timestep that is many times larger than ones liat an explicit code can use. All variables are advanced simultaneously to avoid any syachrunization errors tiat may occur in a staggered $t$ im step scheme.

The targe timestep sizes allowed by the DGA pose problems of their own. Since the MHD equations are nonlinear, we arrive, after ipatial and temporal discretization, at a system of nonlinear algebraic equations that musc be solved. This system is solved via a Newton-Raphson iteration procedure. The iterations continuc until a convergence criterion is met. This process involves the calculation and inversion of the Jacobian of the system. The Jacobian calculation requites the analytic evaluaticn of a number of functional derivatives of the finite difference equations at all the mesh puints. This calculation is carried out on the REDLCE 53 algebraic processor. The REDUCE program is given the finite difference equations and is programmed to gen"rate the Jacobian that:ix elements. The resultant matrix elements are then oucput as a FORTRAN subroutine for use in the main 
code. This procedure has proven to be extremely useful as it has allowed the testing of a number of different fintte difference representations. Without the REDUCE generated Jacoabian subroutine the evaluation of these coefficients would have required many weeks of work to correctly derive and enter into the computer. A discussion of the iteration techniques can be found in Section IJI.5 while Appendix B contains a 1 isting of the REDUCE program used to generate the Jacohian matrix.

A second problem posed by the large timestep allowed by the implicic algorithm is that of selecting the correct timestep size to use. Clearly, if orie i'ses too large a timestep, the results will be inc . cect. Also, since the problem is nonlinear, there is no guarantee that the timestep chosen will lead to a stable time advancement algorithm. Yet, if one uses a very conservative timestep, the efficiency of the 1mplicit algorithm is lowered--perhaps to the point where an explicit scheme is more efficient. The dynamic nature of the problems being run is such that, during their early stages the problems are nearly linear and, therefore, the linear stability analysis should prove to be correct. However, in their latter stages, the problems are highly nonlinear and the results of linear stability analysis do not apply. In order to prevent the early timesteps from being limited by the latter conditions and to allow the physics occurring in the problem to dictate the timestep size chosen, a set of dynamic timestep controls are incorporated in IMP. These are described in Section III. 7.

The boundary conditsons implemented in the code are quite general. Any type of Neumann or Dirichlet or mixed boundary condition is allowed. 
The conservation boundary condition outlined by Lindemuth, 54 which is based on the wall being a flux surface of the plasma, and a pseudo-circuit equation boundary condition are also implemented.

Current versions of IMP do not allow for a concentration point in the coordinates (e.g., $\mathrm{r}=0$ in cylindrical coordinates). For this reason simulations are carried out in rectangular cross section pipes and tori. This aspecí of IMP is not a serious shortcoming and parallels the work of Wooten et al, and Sykes and Wesson.

The code is applied to the study of internal kink modes in varying geometriss. These are the most dangerous modes to plasma stability as they cuuse a gross motion of the plasma colum. Recently, theories have been put forth which relate the internal kink to the "sawtooth-osctllations" or internal disruptions that are observed in the soft $x$-ray emissions from Tokamak discharges. 46

In Chapter IV, a torus is approximated in Cartesian coordinates with a straight rectangular pipe. A great deal of work has veen done in this geometry and provides a hasis for comparison. Agreement is found with the linear growth rates calculated by the Bateman ideal MHD linear instability code. These linear eigenmodes are then allowed to evolve untii they eventually saturate. The saturation amplitudes of the ideal modes agree with the nonlinear calculations of Hicks et a1. 55 In IMP, the linear eigenmodes generated by the Bateman code are used as the initial perturbations and display an Immediate exponential growth. This growth implies that the 
eigenmodes found by using small perturbations in IMP are in close agreement with the eigenmodes determined by the linear code. Next, a fintte resistivity is added to the problem. The modification of growth rates as a function of the magnetic Reynolds number for a particular wavelength instability is shown.

In Chapter $V$, we consider a square cross section torus which is represented in a cylindrical coordinate system. Agreement is found in the fort: and growth rates of the linear, Ideal eigenfunctions. Finite reststiv: $\cdots$ is once again added. $A m=1 / n=1$ mode 15 allowed to evolve in an $s=10^{5}$ plasma for over 1000 Alfven transit times. The $1 / 1$ mode 1 s observed to grow initially, followed by saturation. The system then relaxes to an axisymmetric equilbrium with $a m=1$ flow field appearing near the outer edge of the torus.

In Chapter VI, another plasma regime, the reversed field pinch (RFP), is investigated. A Cartestan grid is used for these simulations. A high pinch ratio, $x\left(x \equiv 2 I / a_{z}\right)$, equilibrium is perturbed in velocity by a $\mathrm{m}=1 / \mathrm{n}=1$ mode. This mode is shown to grow and to cause a spontaneous reversal of the $B_{z}$ fleld in the outer reglons of the plasma. This field reversal increases the shear and stabilizes the kink.

Next, a low pinch ratio Equilfbrium (the same one used in Chapter IV) is raised to the high pinch regime by imposing an external electric field along the walls of the pipe. The externa! fleld causes the current to increase In the plasma, which then pincies 1tself to the higher $x$ regime. A von Neumann ${ }^{56}$ artificlal viscosity is added to stabilize numertc probems 
caused by the compression of the plasma. Once again, an internal kink mode causes the spontaneous field reversal.

Appendix A details a two-dimensional calculation of the resistive sieet pinch. This calculation, which has been performed by Schnack, leads to a strictly resistive instability--the resistive tearing mode (e.g., Furth, Killeen, and Rcsenbluth). ${ }^{57}$ The problem is run in all three possible planes and has verified the correct inclusion of the resistive terms in IMP. The power of the Newton-Raphson Iterations, coupled with the dynamic timestep controls, has allowed IMP to perform the calculation at timesteps one hundred times larger than those used by Schnack.

In all the aforementioned problems, IMP was able to use tinesteps ranging from 5 to 150 times larger than the linear Courant limit--the largest possible timestep an explicit calculation can utilize. 


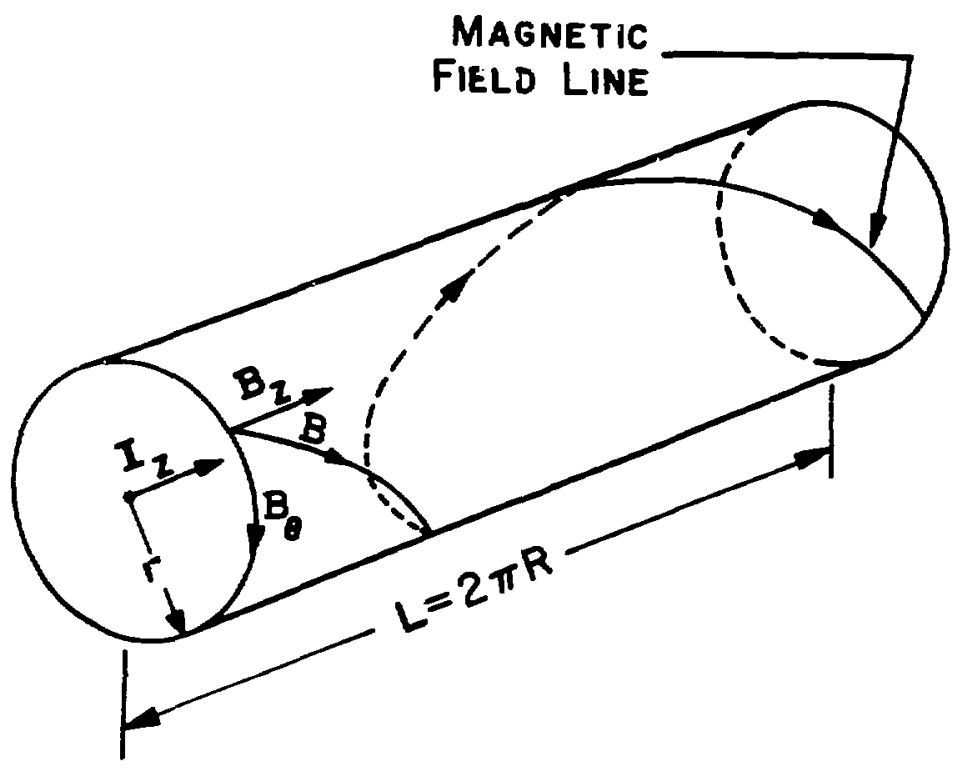

Figure I.I A magnetic ffeld line that is wound on a cylfndrical flux surface. 
$-23-$

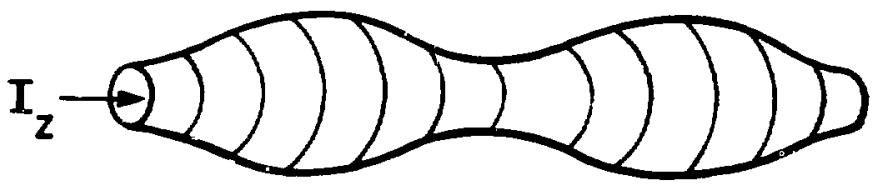

Figure I.2 The $m=0$ sausage mode. 


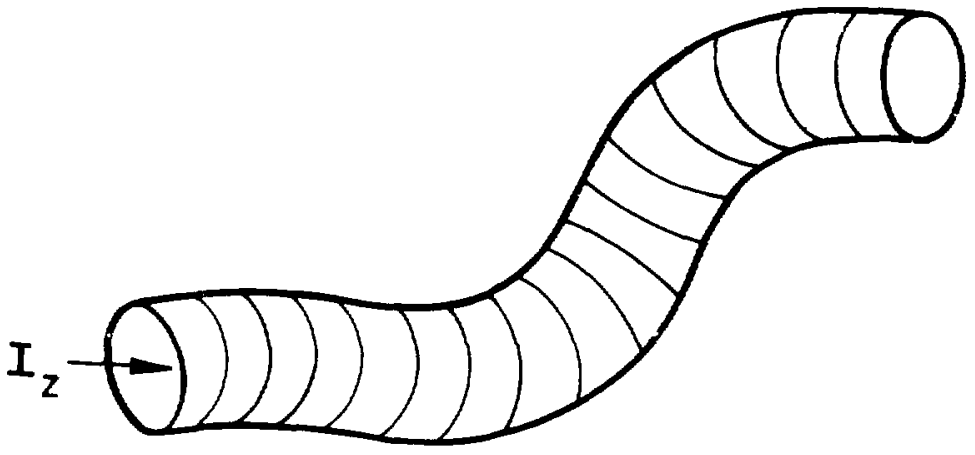

F1gure 1.3 The $m=1$ kink mode. 


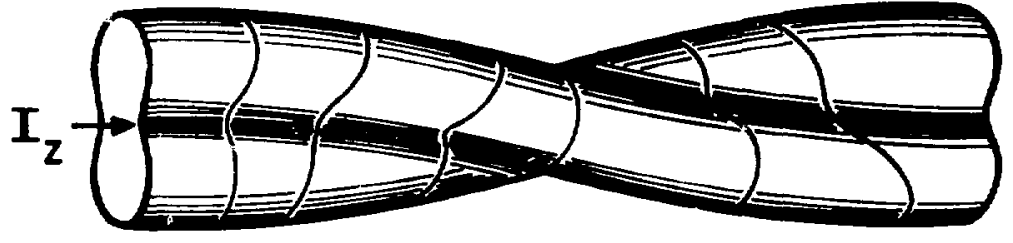

Figure 1.4 The $m=2$ flute mode. 


\section{CHAPTER II}

MATHEMATICAL MODEL

\section{II.1 Introduction}

The single fluid, resistive MHD equations implemented in the IMP code wi1l be presented in this chapter. A heurlstic derlvation will be glven to point out the approximations that are being made. Next, we will show how to cast the equations in conservation form. Then we explain why we chose not to use this computational technique. Finally, we will normalize the vector equations and expand them in orthogonel curvilinear coordinates to obtain the set of equations that we use to follow plasma behavior in IMP.

\section{2 Resistive Single Fluid MHD Equations}

The MHD equations describe the behavior of an electrically neutral conducting fluld in the presence of electroma netic fields and also describe self-consistently the effect that the riluid has on the fie?ds. The macroscopic varlables that descrlbe the fluld are a density $\rho(\underline{x}, t)$, a velocity $\underline{v}(\underline{x}, t)$, and an internal energy $\in(\underline{x}, t)$. We also require the assoclated electromagnetic quantitles: the electric field $\underline{E}(\underline{x}, t)$; the magnetlc flux density $\underline{B}(\underline{x}, t)$; the charge density $q(\underline{x}, t)$; and the current density $\dot{\perp}(\underline{x}, t)$. The equations of a nonconducting invisid, compressible fluld must be modifled to add in electromagnetic effects.

The first fluid equation expresses conservation of mass

$$
\frac{\partial \rho}{\partial t}+\nabla \cdot(\rho \underline{v})=0
$$

and is correct in the absence of sources of mass. 
The equation of motion

$$
\rho \frac{d \underline{v}}{d t} \equiv \rho\left(\frac{\partial \underline{v}}{\partial t}+\underline{v} \cdot \nabla \underline{v}\right)=. \nabla P+\frac{1}{c} j \times \underline{B}
$$

requires an additional force term, the Lorentz force, to account for the electrumagnetic forces of the field on the conducting fluid. Notice the absence of the $q E$ term in the Lorentz force. Th1s is dropped by the assumption of charge neutrality $(q=0)$. In Eulerlan (fixed reference frame) form (after expansion of the convective derivative and substitution of Eq. II.2.1 for $\left.\frac{\partial \rho}{\partial t}\right)$, Eq. II.2.2 becomes

$$
\frac{\partial}{\partial t}(\rho \underline{v})+\nabla \cdot(\rho \underline{v} \underline{v})+\nabla P-\frac{1}{c} j \times \underline{B}=0
$$

The internal energy equation requires the incluston of an additional term due to ohmic heating, $1 . e .$, the heating of the resistive fluid due to the current flowing in it and becomes

$$
\frac{\partial \rho \varepsilon}{\partial t}+\nabla \cdot(\rho \varepsilon \underline{v})+P \nabla \cdot \underline{v}-\pi j^{2}=0
$$

We also need equations expressing the effects of the fluid on the electromagnet1c fields. The fields are described by Maxwell's equations:

$$
\begin{aligned}
& \nabla \times \underline{B}=\frac{4 \pi}{c} j+\frac{1}{c} \frac{\partial E}{\partial t} \\
& \nabla \times \underline{E}=-\frac{1}{c} \frac{\partial B}{\partial t}
\end{aligned}
$$


In the MHD approximation, we assume that the displacement current in Ampere's law is of order $v^{2} / c^{2}$ and, since our flows are to be nonrelativistic, may be 1 gnored. The requirement that $\nabla \cdot \underline{B}=0$ follows from (II.2.5.b) If we impose $\nabla \cdot B=0$ inftially. In order to determine the electric fleld $\underline{E}$, we use Ohm's law in the form

$$
\eta \dot{L}=\underline{E}+\frac{\underline{I}}{c} \underline{v} \times \underline{B}
$$

We now have a set of 14 equations in 15 unknowns: $i, \underline{E}, \underline{\mathbf{v}}, \mathrm{D}, \underline{\mathrm{B}}, E, P$. The system is closed by the provision of an equation of state which relates the pressure, $P$, to the internal energy, $E$, and density, $D$. The plasma is considered an ideal monatomic gas which gives us

$$
P(\varepsilon, \rho)=(\gamma-1) p \varepsilon,
$$

winere $Y=c_{p} / c_{v}$ is the ratio of the specific heats of the plasma. We have now a comp]ete, though unwleldy, system of 15 equations in 15 unknowns.

This system is reduced in the following manncr. The current density i Is eliminated from Ohm's law (II.2.6) by the use of Eq. II.2.5.a. The resulting relationship for the electric field $\underline{E}$ is then substituted into Eq. II.2.5.b ylelding

$$
\frac{\partial B}{\partial t}+\nabla \times\left\{(\underline{v} \times \underline{B})-\frac{c^{2} n}{4 \pi} \nabla \times \underline{B}\right\}=0 \text {. }
$$


The momentum equation (II.2.3) also has $\mathrm{j}$ eliminated via Eq. II.2.5.a. This gives

$$
\frac{\partial \rho \underline{v}}{\partial t}+\nabla \cdot\left(\rho \underline{\underline{v}} \underline{\underline{v}}+\nabla \mathrm{P}+\frac{1}{4 \pi} \underline{B} \times(\nabla \times \underline{\underline{P}})=0\right.
$$

The continuity equation (II.2.1) remains

$$
\frac{\partial \rho}{\partial t}+\nabla \cdot(\rho \underline{v})=0
$$

Finally, the Internal energy $\varepsilon$ in Eq. II.2.4 is replaced with the pressure by using the equation of state (II.2.7) and the current denstty 1 is once agaln replaced via Eq. II.2.5.a, ylelding

$$
\frac{\partial P}{\partial t}+\nabla \cdot(P \underline{y})+(\gamma-1)\left(P \nabla \cdot \underline{v}-\frac{c^{2} \eta}{(4 \pi)^{2}}(\nabla \times \underline{B})^{2}\right)=0
$$

After using the vector 1 dentity $\nabla \cdot A \underline{B}=A \nabla \cdot \underline{B}+\underline{B} \cdot \nabla A$, this becomes

$$
\frac{\partial P}{\partial t}+\nabla \cdot(\gamma P \underline{v})-(\gamma-1)\left\{\underline{v} \cdot \nabla P-\frac{c^{2} n}{(4 \pi)^{2}}(\nabla \times \underline{B})^{2}\right\}=0 \text {. }
$$

Equations (II.2.8) are a system of 8 equations in 8 unknowns.

\section{3 Normalization}

Our next step is to normalize the equations. We introduce a set of nondimenstonal varfables where the 0 subscripted quantities are character- 
istic values for our system,

$$
\begin{aligned}
& \underline{x}+\underline{x}^{\prime} x_{0}, \underline{B}+\underline{B}^{\prime} B_{0}, \rho+\rho^{\prime} \rho_{0}, \\
& \underline{v}+\underline{v}^{\prime} v_{0}, \quad P+P^{\prime} \underline{P}_{0}, t+t^{\prime} t_{0}, \eta+\eta^{\prime} \eta_{0}
\end{aligned}
$$

We then rewrite (II.2.9.a) as

$$
\frac{B_{0}}{t_{0}} \frac{\partial \underline{B}^{\prime}}{\partial t^{\prime}}+\frac{v_{0} B_{0}}{x_{0}} \nabla^{\prime} \times\left(\underline{y}^{\prime} \times \underline{B}^{\prime}\right)-\frac{c^{2} \eta_{0}}{4 \pi x_{0}^{2}} \nabla \times n^{\prime}\left(\nabla \times \underline{B}^{\prime}\right)=0
$$

By letting $x_{0}=v_{0} t_{0},(I I .3 .1)$ simplifies to

$$
\frac{\partial \underline{B}^{\prime}}{\partial t}+\nabla^{\prime} \times\left(\underline{v}^{-} \times \underline{B}^{\prime}\right)-\frac{1}{S} \nabla \times n^{-}\left(\nabla^{-} \times B^{\prime}\right)=0
$$

where $s \equiv \frac{4 \pi v_{0} x_{0}}{c^{2} n_{0}}$. By choosing $v_{0}=v_{A}$ (the Alfven veloctty) $\equiv B_{0} / \sqrt{4 \pi D_{0}}$ and $P .=B_{0}^{2} / 8 \pi=\frac{1}{2} D_{0} v_{A}^{2}$, we may define the Alfven transit time $\tau_{A}=v_{A} / x_{0}$ anc the reststive diffusion time $\tau_{R}=4 \pi x_{0}^{2} / c^{2} n$. Thus $S$ is the wagnetic Reynolds number $\tau_{R} / \tau_{A}$. This gives us a measure of the ratio of the resistive timescale to the Alfven timescale. Next, we define $t_{0}$ such that an Alfven wave travels a distance $x_{0}$ in 1 unit of time, where $x_{0}$ is generally dictated by the dimensions of the probler. When we apply the above procedure to the rest of the equations in (II.2.8), we arrive at the set of normalized MHD equations that are modeled in the IMP code:

$$
\frac{\partial \underline{B}}{\partial t}-\nabla \times\left(\underline{y} \times \underline{B}-\frac{n}{\bar{n}} \nabla \times \underline{B}\right)=0
$$




$$
\begin{aligned}
& \frac{\partial \rho \underline{v}}{\partial+t}+\nabla \cdot(\rho \underline{\underline{v}} \underline{v})+\frac{1}{2} \nabla P+\underline{B} \times(\nabla \times \underline{B})=0 \\
& \frac{\partial \rho}{\partial t}+\nabla \cdot(f \underline{v})=0 \\
& \frac{\partial P}{\partial t}+\nabla \cdot(\gamma P \underline{v})-(\gamma-1)\left\{\underline{v} \cdot \nabla P+\frac{2 n}{s}(\nabla \times \underline{B})^{2}\right\}=0
\end{aligned}
$$

\section{II.4 An Alternate Mode1}

The above set of equations are not all in conservation form. An equation is sald to be in conservation forw when it has one of the following, forms:

a) a scalar conservation law

$$
\frac{\partial \rho}{\partial t}=-\nabla \cdot \underline{F}
$$

b) a vector conservation law

$$
\frac{\partial \underline{v}}{\partial t}=-\nabla \cdot \underline{\underline{q}}
$$

or

c) a pseudovector conservation law

$$
\frac{\partial \underline{\underline{W}}}{\partial t}=\nabla \times \underline{G}
$$

where $\rho$ is a scalar; $\underline{F}, \underline{v}$ and $\underline{G}$ are vectors; $\underline{w}$ is a psevdovector; and $\underline{T}$ symmetric second rank tensor (a dyad). F is the flux of o into the volume 
element and $\underline{\underline{T}}$ is the flux of $\underline{\mathrm{v}}$ 1nto $1 \mathrm{t}$. In the case of Eq. II.4.3, $\underline{w}$ 1s termed a fiux density in the volume element and its varlation is driven by the circulation of the vector $\underline{G}$ around the element rather tinan the amount of G flowing into or out of it. The use of conservation form is common in computer cudes because the calculation of the conservation sums,

$$
\begin{aligned}
& M=\int_{V} \rho d \tau \\
& V=\int_{V} V d \tau
\end{aligned}
$$

and

$$
\phi=\int_{S} \underline{w} \cdot d \underline{a},
$$

gives a convenient test of the consistency of one's coding. It also ensures that no nonphysical sources or sinks of mass, momentum or energy exist. The continuity equation

$$
\frac{\partial \rho}{\partial t}+\nabla \cdot(\rho \underline{v})=0
$$

is in strict conservation form. The magnetic flux equation

$$
\frac{\partial \underline{B}}{\partial t}-\nabla \times\left(\underline{v} \times \underline{B}-\frac{n}{S} \nabla \times \underline{B}\right)=0
$$

Is also in conservation form, although here we have a pseudovector conservation rather than the normal scalar or vector conservation (see Schaack ${ }^{38}$ 
for further deta1ls). Equation (II.3.3.b), the momentum equation, could be converted to conservation form by adding $\underline{B}(\nabla \cdot \underline{B})=0$ ylelding

$$
\frac{\partial \underline{\underline{v}}}{\partial t}+\nabla \cdot\left(p \underline{v} \underline{v}-\underline{B} \underline{B}+\frac{1}{2}\left(P+B^{2}\right) \underline{I}\right)=0
$$

This addition has not been done because, on a finite dffference mesh, $\nabla \cdot \underline{B}$ js not necessartly zero-even if the analytic $\underline{B}$ fleld does correctly display this property. This error arises from the fact that the numeric derivatives are only accurate $t$ order $\Delta x^{2}$. We are thus adding this extra error into our equations for advancing the state varlables ( $\underline{B}, \rho, \underline{v}, P$ ), giving us a feedback of error--the $\nabla \cdot \underline{\underline{R}}$ error induces extra errors in $\underline{v}$ and the extra errors in $\underline{y}$ induce errors in $\underline{B}$ which, In turn, magnify the errors in $\nabla \cdot B--a$ vicious cycle. Runs have been made comparing the two forms of the momentum equation and they show much better numerical results for the nonconservation form of the equation. We achieved much larger time steps with greatly reduced nofse. The recognition of this source of error has been independently arrived at by Brackhill and Barnes. 58,59

The pressure equation (II.3.3.d) is also not in conservation form. If we define in our normalized variables a total energy density $w$ as

$$
w \equiv \frac{1}{2} \rho v^{2}+B^{2}+P /(\gamma-1)
$$

and combine the set of MHD equations as

$$
\underline{B} \cdot \frac{\partial \underline{\underline{B}}}{\partial t}-\frac{1}{2}|\underline{\mathbf{v}}|^{2} \frac{\partial \rho}{\partial t}+\frac{\partial(\rho \varepsilon)}{\partial t}+\underline{v} \cdot \frac{\partial \underline{\underline{v}}}{\partial t} \text {, }
$$


we obtain

$$
\frac{\partial w}{\partial t}+\nabla \cdot\left[(w+P) \underline{v}+\left(B^{2} \underline{I}-2 \underline{B} B\right) \cdot \underline{v}+\frac{2 \eta}{S}(\underline{B} \cdot \nabla \underline{B}-\nabla \underline{B} \cdot B)\right]=0 \quad(I I \cdot 4 \cdot 10)
$$

This form of the equation is not used because now the pressure becomes a subsidary variable,

$$
P=(r-1)\left(w-B^{2}-p v^{2}\right)
$$

When the plasma $B\left(=P / B^{2}\right)$ Is small, the error in the pressure calculition becomes equal to or greater than the calculated pressure. In this form, then, low $\beta$ plasmas cannot be simulated. Total energy conservation is used, Instead, as a check on the accuracy of, rather than as a requirement of, the solution.

\section{II.5 Orthogonal Curvilinear Coordinates}

As we stated in Chapter I, we wish to apply our MHD model to problems in varying coordinate systems. Rather than having to generate the equations for each coordinate system of interest, we adopt the procedure of Schnack ${ }^{38}$ and expand the equations (II.3.3) Ir orthogonal curvilinear coordinates. In this thesis, we extend the work of Schnack to allow vartations in all three coordinate directions. The " $h$ " factors are then calculated in a FUNCTION subroutine so that we may simply vary the subroutine to run in different coordinate systems. 
In orthogonal curvilinear coordinates, we take the differenti al arc length to be

$$
d S^{2} \equiv h_{1}^{2} d x_{1}^{2}+h_{2}^{2} d x_{2}^{2}+h_{3}^{2} d x_{3}^{2}
$$

where the terms $h_{1} d x_{1}$ measure the differential arc length in the $\hat{e}_{1}$ direction. In this system, the metrlc $\mathrm{g}$ is glven by

$$
g=\left(h_{1} h_{2} h_{3}\right)^{2}
$$

and the volume element by $d \tau=h_{1} h_{2} h_{3} d x_{1} d x_{2} d x_{3}$. We also have the relations for the unit vectors $\hat{e}$

$$
\hat{\mathrm{e}}_{1} \cdot \hat{\mathrm{e}}_{\mathrm{j}}=\delta_{1 j}
$$

where $\delta_{\text {if }}$ is the Kronecker delta and

$$
\hat{e}_{1} \times \hat{e}_{j}=E_{1 j k} \hat{e}_{k}
$$

where $E_{1 j k}$ is the Levi-Civita permutation symbol which is defined as

$$
E_{1 j k}=\left\{\begin{aligned}
+1 \quad \text { If }(1 j k)= & (123),(312)(231)(1 . e ., \text { even permutations } \\
& \text { of }(i j k) \\
-1 \text { if }(i j k)= & \text { odd permutations of }(1 j k) \\
0 \quad & \text { otherwise }
\end{aligned}\right.
$$


In this system the gradlent operator becomes

$$
\nabla \equiv \frac{\hat{e}_{1}}{h_{1}} \frac{\partial}{\partial x_{1}}+\frac{\hat{e}_{2}}{h_{2}} \frac{\partial}{\partial x_{2}}+\frac{\hat{e}_{3}}{h_{3}} \frac{\partial}{\partial x_{3}}=\sum_{1} \frac{\hat{e}_{1}}{h_{1}} \frac{\partial}{\partial x_{1}}
$$

where the summation runs from 1 to 3 . In these terms, we may now define the gradient of a scalar and the divergence and curl of a vector.

The gradient of a scalar $\psi$ is

$$
\nabla \psi=\sum_{1} \frac{1}{h_{i}} \frac{\partial \psi}{\partial x_{i}} \hat{e}_{1} .
$$

The divergence of a vector $\underline{v}$ is

$$
\nabla \cdot \underline{v}=\sum_{i} \frac{1}{\sqrt{g}} \frac{\partial}{\partial x_{1}}\left(\sqrt{g} \frac{v_{1}}{h_{1}}\right) .
$$

The curl of a vector $\underline{V}$ is

$$
\nabla \times \underline{v}=\sum_{1} \frac{h_{1} \hat{e}_{1}}{\sqrt{g}} \sum_{\ell, m} E_{i \ell m} \frac{\partial h_{m} v_{m}}{\partial x_{\ell}} .
$$

This is expanded for clarity to give

$$
\begin{aligned}
(\nabla \times \underline{v}) & =\frac{\hat{e}_{1}}{h_{2} \hat{h}_{3}}\left[\frac{\partial}{\partial x_{2}}\left(h_{3} v_{3}\right)-\frac{\partial}{\partial x_{3}}\left(h_{2} v_{2}\right)\right] \\
& +\frac{\hat{e}_{2}}{h_{1} h_{3}}\left[\frac{\partial}{\partial x_{3}}\left(h_{1} v_{1}\right)-\frac{\partial}{\partial x_{1}}\left(h_{3} v_{3}\right)\right] \\
& +\frac{\hat{e}_{3}}{h_{1} h_{2}}\left[\frac{\partial}{\partial x_{1}}\left(h_{2} v_{2}\right)-\frac{\partial}{\partial x_{2}}\left(h_{1} v_{1}\right)\right] .
\end{aligned}
$$


The final relation we need is the divergence of a tensor $\underline{\underline{T}}$. This is given by $^{60}$

$$
(\nabla \cdot \underline{\underline{T}})_{j}=\sum_{1} h_{j} \hat{e}_{j} t_{, j}^{1 j}=\sum_{1} \frac{1}{\sqrt{g}} \frac{\partial}{\partial x_{1}}\left(\sqrt{g} t^{1 j}\right)+\sum_{k} t^{i k}\left\{\begin{array}{l}
j \\
k i
\end{array}\right\} \text { (II.5.10) }
$$

where $t^{i j}=T_{i j} / h_{1} h_{j}$ is the contravarient form of the tensor $I_{1 j}$ and $t_{, j}$ is the covarient derivative of $t .\left\{\begin{array}{l}j \\ i k\end{array}\right\}$ is a Christoffel symbol whose properties are

$$
\begin{aligned}
& \left\{\begin{array}{l}
1 \\
i i
\end{array}\right\}=\frac{1}{h_{i}} \frac{\partial h_{1}}{\partial x_{1}} ;\left\{\begin{array}{l}
1 \\
1 j
\end{array}\right\}=\left\{\begin{array}{l}
1 \\
j 1
\end{array}\right\}=\frac{1}{h_{1}} \frac{\partial h_{1}}{\partial x_{j}} ; \\
& \left\{\begin{array}{l}
j \\
i i
\end{array}\right\}=-\frac{h_{1}}{\left(h_{j}\right)^{2}} \frac{\partial h_{i}}{\partial x_{j}} ;\left\{\begin{array}{l}
1 \\
j k
\end{array}\right\}=0 \text { for } i, j, k \text { all different. }
\end{aligned}
$$

The Christoffel symbols give the change in directions of the unit vectors as one moves in space, and vanish for Cartesian coordinates, We will expand, for clarity, the first component of $\nabla \cdot T$ to shsw the actual form of these terms

$$
\begin{aligned}
(\nabla \cdot \underline{T})_{1} & =\frac{1}{h_{2} h_{2} h_{3}}\left\{\frac{\partial}{\partial x_{1}}\left(h_{2} h_{3} T_{11}\right)+\frac{\partial}{\partial x_{2}}\left(h_{1} h_{3} T_{21}\right)\right. \\
& +\frac{\partial}{\partial x_{3}}\left(h_{1} h_{2} T_{31}\right)+h_{3} \frac{\partial h_{1}}{\partial x_{2}} T_{12}+h_{2} \frac{\partial h_{1}}{\partial x_{3}} T_{13} \\
& \left.-\left[h_{3} \frac{\partial h_{2}}{\partial x_{1}} T_{22}+h_{2} \frac{\partial h_{3}}{\partial x_{1}} T_{33}\right]\right\}
\end{aligned}
$$

Equation (II.5.10) contains terms which look Iike the rormal vector divergence terms plus a set of extra terms that arise due to the change In 
$-38-$

direction of the unit vectors with changes in position. These are called the Coriolis terms.

We may now write down the equations (II.3.3) in component form.

$$
\begin{aligned}
\frac{\partial B_{1}}{\partial t}+ & \frac{1}{h_{2} h_{3}}\left[\frac { \partial } { \partial x _ { 2 } } \left(h _ { 3 } \left\{B_{1} v_{2}-B_{2} v_{1}-\frac{r_{1}}{S} \frac{1}{h_{1} h_{2}}\left\{\frac{\partial}{\partial x_{2}}\left(h_{1} B_{1}\right)\right.\right.\right.\right. \\
& \left.\left.\left.-\frac{\partial}{\partial x_{1}}\left(h_{2} B_{2}\right)\right\}\right\}\right)+\frac{\partial}{\partial x_{3}}\left\{h _ { 2 } \left\{B_{1} v_{3}-B_{3} v_{1}\right.\right. \\
& \left.\left.\left.-\frac{n}{s} \frac{1}{h_{1} h_{3}}\left\{\frac{\partial}{\partial x_{3}}\left(h_{1} B_{1}\right)-\frac{\partial}{\partial x_{1}}\left(h_{3} B_{3}\right)\right\}\right\}\right)\right]=0
\end{aligned}
$$

$$
\begin{aligned}
\frac{\partial B_{2}}{\partial t}+ & \frac{1}{h_{1} h_{3}}\left[\frac { \partial } { \partial x _ { 1 } } \left(h _ { 3 } \left\{B_{2} v_{1}-B_{1} v_{2}-\frac{\eta}{s} \frac{1}{h_{1} h_{2}}\left\{\frac{\partial}{\partial x_{1}}\left(h_{2} B_{2}\right)\right.\right.\right.\right. \\
& \left.\left.-\frac{\partial}{\partial x_{2}}\left(h_{1} B_{1}\right)\right\}\right\}+\frac{\partial}{\partial x_{3}}\left(h _ { 1 } \left\{B_{2} v_{3}-B_{3} v_{2}\right.\right. \\
& \left.\left.-\frac{\eta}{S} \frac{1}{h_{2} h_{3}}\left\{\frac{\partial}{\partial x_{3}}\left(h_{2} B_{2}\right)-\frac{\partial}{\partial x_{2}}\left(h_{3} B_{3}\right)\right\}\right)\right]=0
\end{aligned}
$$

$$
\begin{aligned}
\frac{\partial B_{3}}{\partial t}+ & \frac{1}{h_{1} h_{2}}\left[\frac { \partial } { \partial x _ { 1 } } \left(h _ { 2 } \left\{B_{3} v_{1}-B_{1} v_{3}-\frac{n}{s} \frac{1}{h_{1} h_{3}}\left\{\frac{\partial}{\partial x_{1}}\left(h_{3} B_{3}\right)\right.\right.\right.\right. \\
& \left.\left.\left.-\frac{\partial}{\partial x_{3}}\left(h_{1} B_{1}\right)\right\}\right\}\right)+\frac{\partial}{\partial x_{2}}\left(h _ { 1 } \left\{B_{3} v_{2}-B_{2} v_{3}\right.\right. \\
& \left.\left.-\frac{n}{s} \frac{1}{h_{2} h_{3}}\left\{\frac{\partial}{\partial x_{2}}\left(h_{3} B_{3}\right)-\frac{\partial}{\partial x_{3}}\left(h_{2} B_{2}\right)\right\}\right)\right]=0
\end{aligned}
$$


$-39-$

$$
\begin{aligned}
\frac{\partial \rho v_{1}}{\partial t} & +\frac{1}{h_{1} h_{2} h_{3}}\left\{\frac{\partial}{\partial x_{1}}\left(h_{2} h_{3} \rho v_{1}^{2}\right)+\frac{\partial}{\partial x_{2}}\left(h_{1} h_{3} \rho v_{1} v_{2}\right)\right. \\
& +\frac{\partial}{\partial x_{3}}\left(h_{1} h_{2} \rho v_{1} v_{3}\right)+h_{3} \rho v_{1} v_{2} \frac{\partial h_{1}}{\partial x_{2}}+h_{2} \rho v_{1} v_{3} \frac{\partial h_{1}}{\partial x_{3}} \\
& \left.-\left[h_{3} \rho v_{2}^{2} \frac{\partial h_{2}}{\partial x_{1}}+h_{2} \rho v_{3}^{2} \frac{\partial h_{3}}{\partial x_{1}}\right]\right\}+\frac{1}{2 h_{1}} \frac{\partial P}{\partial x_{1}} \\
& +\left[\frac{B_{2}}{h_{1} h_{2}}\left\{\frac{\partial}{\partial x_{1}}\left(h_{2} B_{2}\right)-\frac{\partial}{\partial x_{2}}\left(h_{1} B_{1}\right)\right\}\right. \\
+ & \frac{B_{3}}{h_{1} h_{3}}\left\{\frac{\partial}{\partial x_{3}}\left(h_{1} B_{1}\right)-\frac{\partial}{\partial x_{1}}\left(h_{3} B_{3}\right)\right\}=0
\end{aligned}
$$

$$
\begin{aligned}
\frac{\partial \rho v_{2}}{\partial t} & +\frac{1}{h_{1} h_{2} h_{3}}\left\{\frac{\partial}{\partial x_{1}}\left(h_{2} h_{3} \rho v_{1} v_{2}\right)+\frac{\partial}{\partial x_{2}}\left(h_{1} h_{3} \rho v_{2}^{2}\right)\right. \\
& +\frac{\partial}{\partial x_{3}}\left(h_{1} h_{2} \rho v_{2} v_{3}\right)+h_{3} \rho v_{1} v_{2} \frac{\partial h_{2}}{\partial x_{1}}+h_{1} \rho v_{2} v_{3} \frac{\partial h_{2}}{\partial x_{3}} \\
& \left.-\left[h_{3} \rho v_{1}^{2} \frac{\partial h_{1}}{\partial x_{2}}+h_{1} \rho v_{3}^{2} \frac{\partial h_{3}}{\partial x_{2}}\right]\right\}+\frac{1}{2 h_{2}} \frac{\partial P}{\partial x_{2}} \\
& +\left[\frac{B_{3}}{h_{2} h_{3}}\left\{\frac{\partial}{\partial x_{2}}\left(h_{3} B_{3}\right)-\frac{\partial}{\partial x_{3}}\left(h_{2} B_{2}\right)\right\}\right. \\
+ & \left.\frac{B_{1}}{h_{1} h_{2}}\left\{\frac{\partial}{\partial x_{1}}\left(h_{2} B_{2}\right)-\frac{\partial}{\partial x_{2}}\left(h_{1} B_{1}\right)\right\}\right]=0
\end{aligned}
$$




$$
\begin{aligned}
\frac{\partial \rho v_{3}}{\partial t} & +\frac{1}{h_{1} h_{2} h_{3}}\left\{\frac{\partial}{\partial x_{1}}\left(h_{2} h_{3} \rho v_{1} v_{3}\right)+\frac{\partial}{\partial x_{2}}\left(h_{1} h_{3} \rho v_{2} v_{3}\right)\right. \\
& +\frac{\partial}{\partial x_{3}}\left(h_{1} h_{2} \rho v_{3}^{2}\right)+h_{2} \rho v_{1} v_{3} \frac{\partial h_{3}}{\partial x_{1}}+h_{1} \rho v_{2} v_{3} \frac{\partial h_{3}}{\partial x_{2}} \\
& \left.-\left[h_{2} \rho v_{1}^{2} \frac{\partial h_{1}}{\partial x_{3}}+h_{1} \rho v_{2}^{2} \frac{\partial h_{2}}{\partial x_{3}}\right]\right\}+\frac{1}{2 h_{3}} \frac{\partial P}{\partial x_{3}} \\
& +\left[\frac{B_{1}}{h_{1} h_{3}}\left\{\frac{\partial}{\partial x_{3}}\left(h_{1} B_{1}\right)-\frac{\partial}{\partial x_{1}}\left(h_{3} B\right)\right\}\right. \\
& +\frac{B_{2}}{h_{2} h_{3}}\left\{\frac{\partial}{\partial x_{3}}\left(h_{2} B_{2}\right)-\frac{\partial}{\partial x_{2}}\left(h_{3} B_{3}\right)\right\}=0
\end{aligned}
$$

$$
\begin{gathered}
\frac{\partial \rho}{\partial t}+\frac{1}{h_{1} h_{2} h_{3}}\left\{\frac{\partial}{\partial x_{1}}\left(h_{2} h_{3} \rho v_{1}\right)+\frac{\partial}{\partial x_{2}}\left(h_{1} h_{3} \rho v_{2}\right)\right. \\
\left.+\frac{\partial}{\partial x_{3}}\left(h_{1} h_{2} \rho v_{3}\right)\right\}=0
\end{gathered}
$$

$$
\begin{aligned}
\frac{\partial P}{\partial t}+ & \frac{\gamma}{h_{1} h_{2} h_{3}}\left\{\frac{\partial}{\partial x_{1}}\left(h_{2} h_{3} P v_{1}\right)+\frac{\partial}{\partial x_{2}}\left(h_{1} h_{3} P v_{2}\right)\right. \\
& \left.+\frac{\partial}{\partial x_{3}}\left(h_{1} h_{2} P v_{3}\right)\right\}-(y-1)\left[\left\{\frac{v_{1}}{h_{1}} \frac{\partial P}{\partial x_{1}}+\frac{v_{2}}{h_{2}} \frac{\partial P}{\partial x_{2}}\right.\right. \\
& \left.+\frac{v_{3}}{h_{3}} \frac{\partial P}{\partial x_{3}}\right\}+\frac{2 \pi}{S}\left\{\frac{1}{\left(h_{2} h_{3}\right)^{2}}\left[\frac{\partial}{\partial x_{2}}\left(h_{3} B_{3}\right)-\frac{\partial}{\partial x_{3}}\left(h_{2} B_{2}\right)\right]^{2}\right. \\
& +\frac{1}{\left(h_{1} h_{3}\right)^{2}}\left[\frac{\partial}{\partial x_{3}}\left(h_{1} B_{1}\right)-\frac{\partial}{\partial x_{1}}\left(h_{3} B_{3}\right)\right]^{2} \\
& \left.+\frac{1}{\left(h_{1} h_{2}\right)^{2}}\left[\frac{\partial}{\partial x_{1}}\left(h_{2} B_{2}\right)-\frac{\partial}{\partial x_{2}}\left(h_{1} B_{1}\right)\right]^{2}\right\}=0
\end{aligned}
$$


These are the equations that IMP uses to simulate plasma behavior. The next chaper will discuss how they are solved. 
CHAPTER III

\section{COMPUTATIONAL TECHNIQUES}

\section{I Introduction}

The nonlinear, time dependent, three-dimensional coupled set of MHD equations that were presented in Chapter II are solved via alternating direction Implicit (ADI) finite difference techniques on a nonuniform Eulerian mesh. In this chapter, the implementation of these techniques in the IMP (Implicit MHD Program) code is discussed, including descriptions of the computational mesh, the ADI algorithm, and the spatial finite differencing. An improved technique for the linearization of the resulting nonlinear algebraic equations is also presented. The solution algorithm for the block tridiagonal matrices that arise from the implicit equations is derlved for both periodic and nomperiodic boundary condtions. The boundary conditions that are implemented in DPP are described. Finally, the timestep size controls that have been implemented are presented.

The MHD equations (II. 3.3) are expressed as

$$
\frac{\partial \underline{u}(\underline{u})}{\partial t}+\underline{F}(\underline{u})+\underline{G}(\underline{u})+\underline{H}(\underline{u})=0
$$

where $\underline{\underline{u}} \equiv\{\underline{B}, \underline{v}, \rho, P\}$ is the state variable of the system and $\underline{U}(\underline{u}) \equiv\{\underline{B}, \rho \underline{v}, \rho, P\}$ are the quantities advanced in time. Notice that even the vector $\underline{U}$ is nonlinear and requires an eventual linearization. The advantage of using $\underline{u}$ as onr state vector rather than using $\underline{U}$ as has been done previously $y^{38}$ is that the velocity no longer needs to lag the solution in either time or iteration space. 
We now de:ine the three functions $\underline{F}, \underline{G}$, and $\underline{H}$. The function $\underline{F}(\underline{u})$ contains all terms in Eqs. II.5.13 that have leading derivatives with respect to $x_{1}$. By leading derivatives, we mean any of the following forms: (1) $\alpha \frac{\partial \phi}{\partial x_{1}}$; (2) $\frac{\partial \alpha \phi}{\partial x_{1}}$; (3) $\alpha \frac{\partial \phi}{\partial x_{1}} \frac{\partial \psi}{\partial x_{2}}$; (4) $\frac{\partial}{\partial x_{1}}\left(\alpha \frac{\partial \phi}{\partial x_{1}}\right)$; or (5) $\frac{\partial}{\partial x_{1}}\left(a \frac{\partial \phi}{\partial x_{2}}\right)$ (note: all $\frac{\partial}{\partial x_{2}}$ 's may be replaced by $\frac{\partial}{\partial x_{3}} \cdot s$ ). Similarly, the functions $\underline{G}(\underline{u})$ and $\underline{H}(\underline{u})$ contain all the terms in Eqs. (II.3.12) that have leading derivatives with respect to $x_{2}$ and $x_{3}$ respectively. In the case of form 3 above, the term is split between both $\underline{F}$ and $\underline{G}$ by setting $\frac{\partial \phi}{\partial x_{1}} \frac{\partial \psi}{\partial x_{2}}=$ $1 / 2\left(\frac{\partial \phi}{\partial x_{1}} \frac{\partial \psi}{\partial x_{2}}+\frac{\partial \psi}{\partial x_{2}} \frac{\partial \phi}{\partial x_{1}}\right)$. The functions, then, are:

$$
\begin{aligned}
& F_{1}(\underline{u})=0 \\
& F_{2}(\underline{u})=\frac{1}{h_{1} h_{3}} \frac{\partial}{\partial x_{1}}\left\{h _ { 3 } \left(B_{2} v_{1}-B_{1} v_{2}-\frac{r_{1}}{S} \frac{1}{h_{1} h_{2}}\right.\right. \\
& \left.\left.x\left[\frac{\partial}{\partial x_{1}}\left(h_{2} B_{2}\right)-\frac{\partial}{\partial x_{2}}\left(h_{1} B_{2}\right)\right]\right)\right\} \\
& F_{3}(\underline{u})=\frac{1}{h_{1} h_{2}} \frac{\partial}{\partial x_{1}}\left\{h _ { 2 } \left(B_{3} v_{1}-B_{1} v_{3}-\frac{n}{s} \frac{l}{h_{1} h_{3}}\right.\right. \\
& \left.\left.x\left[\frac{\partial}{\partial x_{1}}\left(h_{3} B_{3}\right)-\frac{\partial}{\partial x_{3}}\left(h_{1} B_{1}\right)\right]\right)\right\} \\
& F_{4}(\underline{u})=\frac{1}{h_{1} h_{2} h_{3}}\left\{\frac{\partial}{\partial x_{1}}\left(h_{2} h_{3} \rho v_{1}^{2}\right)-\left[h_{3} \rho v_{2}^{2} \frac{\partial h_{2}}{\partial x_{1}}\right.\right. \\
& \left.\left.+h_{2} \rho v_{3}^{2} \frac{\partial h_{3}}{\partial x_{1}}\right]\right\}+\frac{1}{2 h_{1}} \frac{\partial P}{\partial x_{1}}+\frac{B_{2}}{h_{1} h_{2}} \frac{\partial}{\partial x_{1}}\left(h_{2} B_{2}\right) \\
& +\frac{B_{3}}{h_{1} h_{3}} \frac{\partial}{\partial x_{1}}\left(h_{3} B_{3}\right)
\end{aligned}
$$




$$
\begin{aligned}
& F_{5}(\underline{u})=\frac{1}{h_{1} h_{2} h_{3}}\left\{\frac{\partial}{\partial x_{1}}\left(h_{2} h_{3} \rho v_{1} v_{2}\right)+h_{3} \rho v_{1} v_{2} \frac{\partial h_{2}}{\partial x_{1}}\right\} \\
& -\frac{\mathrm{B}_{1}}{{ }_{\mathrm{h}_{1} \mathrm{~h}_{2}}} \frac{\partial}{\partial \mathrm{r}_{1}}\left(\mathrm{~h}_{2} \mathrm{~B}_{2}\right) \\
& F_{6}(\underline{u})=\frac{1}{h_{1} h_{2} h_{3}}\left\{\frac{\partial}{\partial x_{1}}\left(h_{2} h_{3} v v_{1} v_{3}\right)+h_{2} \nu v_{1} v_{3} \frac{\partial h_{3}}{\partial x_{1}}\right\} \\
& -\frac{B_{1}}{h_{1} h_{3}} \frac{\partial}{\partial x_{1}}\left(h_{3} B_{3}\right) \\
& F_{7}(\underline{u})=\frac{1}{h_{1} h_{2} h_{3}} \frac{i}{3 x_{1}}\left(h_{2} h_{3}+v_{1}\right) \\
& F_{8}(\underline{u})=\frac{r}{h_{1} h_{2} h_{3}} \frac{\partial}{\partial x_{1}}\left(h_{2} h_{3} P v_{1}\right)-(\gamma-1)\left[\frac{v_{1}}{h_{1}}{ }_{\partial x_{1}} p\right. \\
& +\frac{2 \pi}{S}\left\{\frac{1}{\left(h_{1} h_{3}\right)^{2}} \frac{\partial\left(h_{1} B_{3}\right)}{\partial x_{1}}\left(\frac{\partial\left(h_{3} B_{3}\right)}{\partial x_{1}}-\frac{\partial\left(h_{1} B_{1}\right)}{3 x_{3}}\right)\right. \\
& \left.\left.+-\frac{1}{\left(h_{1} h_{2}\right)^{2}} \frac{\partial\left(h_{2} B_{2}\right)}{\partial x_{1}}\left(\frac{\partial h_{2} B_{2}}{\partial x_{1}}-\frac{\partial\left(h_{1} B_{1}\right)}{\partial x_{2}}\right)\right\}\right]
\end{aligned}
$$

and

$$
\begin{aligned}
G_{1}(\underline{u}) & =\frac{1}{h_{2} h_{3}} \frac{\partial}{\partial x_{2}}\left\{h _ { 3 } \left(B_{1} v_{2}-B_{2} v_{1}-\frac{\eta}{S} \frac{1}{h_{1} h_{2}}\left[\frac{\partial}{\partial x_{2}}\left(h_{1} B_{1}\right)\right.\right.\right. \\
& \left.\left.\left.-\frac{\partial}{\partial x_{1}}\left(h_{2} B_{2}\right)\right)\right\}\right]
\end{aligned}
$$


$-45-$

$$
G_{2}(\underline{u})=0
$$

$$
\begin{aligned}
G_{3}(\underline{u}) & =\frac{1}{h_{1} h_{2}} \frac{\partial}{\partial x_{2}}\left\{h _ { 1 } \left(B_{3} v_{2}-B_{2} v_{3}-\frac{n}{s} \frac{1}{h_{2} h_{3}}\left[\frac{\partial}{\partial x_{2}}\left(h_{3} B_{3}\right)\right.\right.\right. \\
& \left.\left.\left.-\frac{\partial}{\partial x_{3}}\left(h_{2} B_{2}\right)\right]\right)\right\}
\end{aligned}
$$

$$
\begin{aligned}
G_{4}(u) & =\frac{1}{h_{1} h_{2} h_{3}}\left\{\frac{\partial}{\partial x_{2}}\left(h_{1} h_{3} \rho v_{1} v_{2}\right)+h_{3} \rho v_{1} v_{2} \frac{3 h_{1}}{\partial x_{2}}\right\} \\
& -\frac{B_{2}}{h_{1} h_{2}} \frac{\partial}{\partial x_{2}}\left(h_{1} B_{1}\right)
\end{aligned}
$$

$$
\begin{aligned}
G_{5}(\underline{u}) & =\frac{j}{h_{1}} \frac{j}{h_{2} i_{3}}\left\{\frac{\partial}{\partial x_{2}}\left(h_{1} h_{3} \rho v_{2}^{2}\right)-\left[h_{3}=v_{1} \frac{\partial \mathrm{h}_{1}}{\partial x_{2}}\right.\right. \\
& \left.\left.+h_{1} \rho v_{3}^{2} \frac{\partial h_{3}}{\partial x_{2}}\right]\right\}+\frac{1}{2 h_{2}} \frac{\partial P}{\partial x_{2}}+\frac{B_{1}}{h_{1} h_{2}} \frac{\partial}{\partial x_{2}}\left(h_{1} B_{1}\right) \\
& +\frac{B_{3}}{h_{2} h_{3}} \frac{\partial}{\partial x_{2}}\left(h_{3} B_{3}\right)
\end{aligned}
$$

$$
\begin{aligned}
G_{6}(\underline{u}) & =\frac{1}{h_{1} h_{2} h_{3}}\left\{\frac{\partial}{\partial x_{2}}\left(h_{1} h_{3} p v_{2} v_{3}\right)+h_{1} r v_{2} v_{3} \frac{\partial h_{3}}{\partial x_{2}}\right\} \\
& -\frac{B_{2}}{h_{2} h_{3}} \frac{\partial}{\partial x_{2}}\left(h_{3} B_{3}\right) \\
G_{7}(\underline{u}) & =\frac{1}{h_{1} h_{2} h_{3}} \frac{\partial}{\partial x_{2}}\left(h_{1} h_{3} \circ v_{2}\right)
\end{aligned}
$$


$-46-$

$$
\begin{aligned}
G_{8}(\underline{u}) & =\frac{\gamma}{h_{1} h_{2} h_{3}} \frac{\partial}{\partial x_{2}}\left(h_{1} h_{3} P v_{2}\right)-(\gamma-1)\left[\frac{v_{2}}{h_{2}} \frac{\partial P}{\partial x_{2}}\right. \\
& +\frac{2 n}{s}\left\{\frac{1}{\left(h_{1} h_{3}\right)^{2}} \frac{\partial\left(h_{3} B_{3}\right)}{\partial x_{2}}\left(\frac{\partial\left(h_{3} B_{3}\right)}{\partial x_{2}}-\frac{\partial\left(h_{2} B_{2}\right)}{\partial x_{3}}\right)\right. \\
& \left.\left.+\frac{1}{\left(h_{1} h_{2}\right)^{2}} \frac{\partial\left(h_{1} B_{1}\right)}{\partial x_{2}}\left(\frac{\partial\left(h_{1} B_{1}\right)}{\partial x_{2}}-\frac{\partial\left(h_{2} B_{2}\right)}{\partial x_{1}}\right)\right\}\right]
\end{aligned}
$$

and, fina11y,

(III.1.4)

$$
\begin{aligned}
\mathrm{H}_{1} \underline{(\mathrm{u})} & =\frac{1}{\mathrm{~h}_{2} \mathrm{~h}_{3}} \frac{\partial}{\partial \mathrm{x}_{3}}\left\{\mathrm { h } _ { 2 } \left(\mathrm{~B}_{1} \mathrm{v}_{3}-\mathrm{B}_{3} \mathrm{v}_{1}-\frac{\mathrm{n}}{\mathrm{s}} \frac{1}{\mathrm{~h}_{1} \mathrm{~h}_{3}}\left[\frac{\partial}{\partial \mathrm{x}_{3}}\left(\mathrm{~h}_{1} \mathrm{~B}_{1}\right)\right.\right.\right. \\
& \left.\left.\left.-\frac{\partial}{\partial \mathrm{x}_{1}}\left(\mathrm{~h}_{3} B_{3}\right)\right]\right)\right\}
\end{aligned}
$$

$$
\begin{aligned}
H_{2}(\underline{u}) & =\frac{1}{h_{1} h_{3}} \frac{\partial}{\partial x_{3}}\left\{h _ { 1 } \left(B_{2} v_{3}-B_{3} v_{2}-\frac{n}{s} \frac{1}{h_{2} h_{3}}\left[\frac{i}{\partial x_{3}}\left(h_{2} B_{2}\right)\right.\right.\right. \\
& \left.\left.\left.-\frac{\partial}{\partial x_{2}}\left(h_{3} B_{3}\right)\right]\right)\right\}
\end{aligned}
$$

$$
\mathrm{H}_{3}(\underline{u})=0
$$

$$
\begin{aligned}
H_{4}(\underline{u}) & =\frac{1}{h_{1} h_{2} h_{3}}\left\{\frac{\partial}{\partial x_{3}}\left(h_{1} h_{2} \rho v_{1} v_{3}\right)+h_{2} \rho v_{1} v_{3} \frac{\partial h_{1}}{\partial x_{3}}\right\} \\
& -\frac{B_{3}}{h_{1} h_{3}} \frac{\partial}{\partial x_{3}}\left(h_{1} B_{1}\right)
\end{aligned}
$$




$$
\begin{aligned}
H_{5}(\underline{u}) & =\frac{1}{h_{1} h_{2} h_{3}}\left\{\frac{\partial}{\partial x_{3}}\left(h_{1} h_{2} \rho v_{2} v_{3}\right)+h_{1} \rho v_{2} v_{3} \frac{\partial h_{2}}{\partial x_{3}}\right\} \\
& -\frac{B_{3}}{h_{2} h_{3}} \frac{\partial}{\partial x_{3}}\left(h_{2} B_{2}\right) \\
H_{6}(\underline{u}) & =\frac{1}{h_{1} h_{2} h_{3}}\left\{\frac{\partial}{\partial x_{3}} h_{1} h_{2} \rho v_{3}^{2}-\left[h_{2} \rho v_{1}^{2} \frac{\partial h_{1}}{\partial x_{3}}\right.\right. \\
& \left.\left.+h_{1} \rho v_{2}^{2} \frac{\partial h_{2}}{\partial x_{3}}\right]\right\}+\frac{1}{2 h_{3}} \frac{\partial P}{\partial x_{3}}+\frac{B_{1}}{h_{1} h_{3}} \frac{\partial}{\partial x_{3}}\left(h_{1} B_{1}\right) \\
& +\frac{B_{2}}{h_{2} h_{3}} \frac{\partial}{\partial x_{3}}\left(h_{2} B_{2}\right)
\end{aligned}
$$

$$
H_{7}(\underline{u})=\frac{1}{h_{1} h_{2} h_{3}} \frac{\partial}{\partial x_{3}}\left(h_{1} h_{2} \rho v_{3}\right)
$$$$
H_{8}(\underline{u})=\frac{\gamma}{h_{1} h_{2} h_{3}} \frac{\partial}{\partial x_{3}}\left(h_{1} h_{2} P v_{3}\right)-(\gamma-1)\left[\frac{v_{3}}{h_{3}} \frac{\partial P}{\partial x_{3}}\right.
$$$$
+\frac{2 n}{S}\left\{\frac{1}{\left(h_{2} h_{3}\right)^{2}} \frac{\partial}{\partial x_{3}}\left(h_{2} B_{2}\right)\left(\frac{\partial}{\partial x_{3}}\left(h_{2} B_{2}\right)-\frac{\partial}{\partial x_{2}}\left(h_{3} B_{3}\right)\right)\right.
$$$$
\left.\left.+\frac{1}{\left(h_{1} h_{3}\right)^{2}}-\frac{\partial}{\partial x_{3}}\left(h_{1} B_{1}\right)\left(\frac{\partial}{\partial x_{3}}\left(h_{1} B_{1}\right)-\frac{\partial}{\partial x_{1}}\left(h_{3} B_{3}\right)\right)\right\}\right]
$$

Careful examination of $\underline{F}, \underline{G}$, and $\underline{H}$ reveals an extremel $;$ useful symmetry. If all 1 subscripts in $\underline{F}$ are interchanged with all 2 subscripts, $\underline{F}$ becomes identical to $\mathrm{G}$. In a similar fashion, a 1,3 interchange on $\underline{F}$ yields $\underline{H}$. We utilize this feature of the equations to greatly simplify our 
coding in IMP. Finally, one component of the magnetic fieid equation is zero in each of the functions, $\underline{F}, \underline{G}$, and $\underline{H}$. This reduces the number of equations that need to be solved simultaneously at each mesh point from 8 to 7 , a savings of $20 \%$ on the work required per mesh point (work scales as $\left.n^{2}\right)$.

\section{I.1.1 Basic Numerical Analys is}

We now introduce some concepts from numerical analysis that will be referred to throughout the remainder of this thests. For ease of notation, this section will deal with representations in only one spatial dimension. The extension of these concepts to two and three spatial dimensions is relatively straightforward and will be deferred to later.

We bagin with a continuous set of [unctions $\underline{U}(x, t)$ which are defined on the domain $\left(x_{1}, x_{2}\right)$. The domain is represented as a set of discrete points $x_{i}, 1 \leq i \leq I$, such that

$$
x_{i}=x_{1}+\sum_{j=1}^{i-j} \Delta x_{j}
$$

where

$$
\Delta x_{j} \equiv x_{j}-x_{j-1}
$$

The points $x_{i}$ form a mesh or a grid. The mesh points themselves may move with some velocity $v_{G}$ with respect to an observer who is stationary in the laboratory frame. If this veloci:y is zero, the 
mesh is called Eulerian. If the velocity $v_{G}$ equais the velocity of the fluid at all points on the mesh, the mesh is called Lagrangian. Since the velocity $v_{G}$ may, in general, vary from the two above limits, a mesh that explicitly contains the grid velocity in its definition is called a generalized mesh.

Time is also discretized so that

$$
t^{n}=t^{\phi}+\sum_{j=0}^{n-1} \Delta t^{j}
$$

where $t^{\phi}$ is some initial time (usually 0 ) and

$$
\Delta t^{j} \equiv t^{j}-t^{j-1}
$$

The continuous functions $\underline{U}(x, t)$ are now expressed as the discrete functions $\underline{u}_{i}^{n} \equiv \underline{v}\left(x_{i}, t^{n}\right)$.

We are interested in solving the initial value problem in which we are given $\underline{U}_{i}^{n}$ for all $i$ and want to know $\underline{U}_{i}^{n+1}$. In order to advance f rom time $t=t^{n}$ and $t=t^{n+1}$ we must solve the system of $m$ coupled partial differential equations

$$
\frac{\partial \underline{U}(x, t)}{\partial t}=\underline{N}(\underline{U}(x, t), x, t)
$$

where $N$ is some set of $m$ (possibily nonlinear) spatial differential operators. This system is replaced by a set of $\mathrm{m} x \mathrm{I}$ (possibly 
coupled) algebraic equations via finite difference techniques. These may be represented by

$$
\begin{aligned}
\underline{u}_{i}^{n+1} & =\underline{u}_{i}^{n}+\Delta t\left((1-\theta)\left(\underline{v}_{i+1}^{n}, \underline{u}_{-1}^{n}, \underline{v}_{1-1}^{n}, x_{1}, t^{n}\right)\right. \\
& \left.+\theta\left(\underline{N} \underline{u}_{i+1}^{n+1}, \underline{u}_{-1}^{n+1}, \underline{v}_{1-1}^{n+1}, x_{i}, t^{n+1}\right)\right)
\end{aligned}
$$

where $0 \leq \theta \leq 1$

If $\theta$ equals zero, then the $\underline{\mathrm{U}}_{i}^{\mathrm{n}+1}$ are not coupled with any other $\underline{\mathrm{U}}^{\mathrm{n}+1}$ values and is an explfclt time advance algorithm. In this case, the advance to the new time level requires only the solution of $m \times I$ uncoupled algebraic equat lons. Since the value of $\underline{u}_{i}^{n+1}$ is a function of only $\underline{v}_{i+1}^{n}, \underline{U}_{1}^{n}, \underline{U}_{i-1}^{n}$, we see that information can only propagate one spatial zone per time advance (timestep). We, then, can expect this to put an upper limit on the size of an expliclt timestep. This Iimit is called a Courant-Fredricks-Lewy (CFL) condition and it must be met in every zone of the problem otherwise numeric Instabilities will ensue.

If we consider Eq. (III.1.1.5) to be simple advection equation

$$
\frac{\partial U}{\partial t}=-v \frac{\partial U}{\partial x}
$$

where $v$ is a constant velocity of the advection, the CFL condition is

$$
\Delta t \leq \frac{\Delta x}{|v|}
$$


This condition ensures that the velocity of the physical process (the advection) is less than the velocity of information transmittal on the mesh. In the case of the MHD equations, the characteristic physical velocity is the Alfuen velocity $\left(v_{A}\right)$ which is proportional to the magnetic flux (B) and inversely proportional to the square root of the density $(\rho)\left(v_{A} a B / \sqrt{\rho}\right)$. In general, areas of high magnetic flux tend to have low densities--thus leading to very large Alfvén velocities. These high flux-low density zones generally are not where the interesting physics is occurring but they can severely limit the allowable explicit timestep.

Similarly, if Eq. (III.1.1.5) is the diffuston equation

$$
\frac{\partial U}{\partial t}=D \frac{\partial^{2} U}{\partial x^{2}},
$$

where $D$ is a constant coefficient of dffusion, the CFL condition is

$$
\Delta t \leq \frac{(\Delta x)^{2}}{2 D} .
$$

In this case, we see that the mesh spacing $\Delta x$ can be a significant limit on the allowable timestep. If we halve the mesh spacing (double the number of mesh points), we cut the maximum allowable timestep by a factor of four. Thus, we must perform eight times as much work to follow the equation for the same cmount of simulation time. 
In order to bypass these strict limitations on timestep size, we may let $\theta$ in Eq. (III.1.1.6) be greater than or equal to one half ( $\odot \geq 1 / 2)$. In this case, the values of $\underline{U}_{i}^{n+1}$ are fully coupled together and the velocity of information transmittal on the mesh is infinite 7 and is termed an implicit time advancement algorithm. In the case of (IJI.1.1.5) being a set of linear equations, any time step size will be stable; and even when $\underline{N}$ in (III.1.1.5) is a nonlinear operator, we may expect an improvement in the maximum allowable timestep size. The price paid here is that the m $x$ I system of equations are coupled which necessitates the inversion of a (n $x$ I) $x$ (m $x$ I) matrix (i.e., $\left.\underline{A} \underline{\mathrm{v}}^{\mathrm{n}+1}=\underline{B}\right)$. This inversion requires of order $(\mathrm{m} x \mathrm{I})^{3}$ arjthmetic operations. 6]. For the MHD equations, $m$ is equal to eight (see Chapter II). If we consider a 100 point mesh, this is $5.12 \times 10^{8}$ operations. A CDC-7600 computer can perform $3 \times 10^{6}$ operations per second which says that we may invert this matrix in a little under 2-1/2 minutes. While this rate seems to be expensive, yet feasible, consider a threedimensional mesh with $20 \times 20 \times 20$ points. Here we would have a 64000 x 64000 matrix ( $\mathrm{m} \times \mathrm{I} \times \mathrm{J} \times \mathrm{K}$ ) to invert--10 $0^{14}$ operations or over 7200 hours per inversion.

These times can be decreased by many orders of magnitude by examining the structure of the matrix $\stackrel{A}{=}$ to be inverted. In the onedimensional case, we see that $\underline{v}_{i}^{n+1}$ is directly coupled only to $\underline{v}_{i+1}^{n+1}$, $\underline{v}_{i}^{n+1}$ and $\underline{v}_{i-1}^{n+1}$. Thus, the matrix $\stackrel{A}{=}$ to be inverted has a block tridiagonal structure (see Fig. III.1), where each block element is a m $x$ m submat $r i x$. 

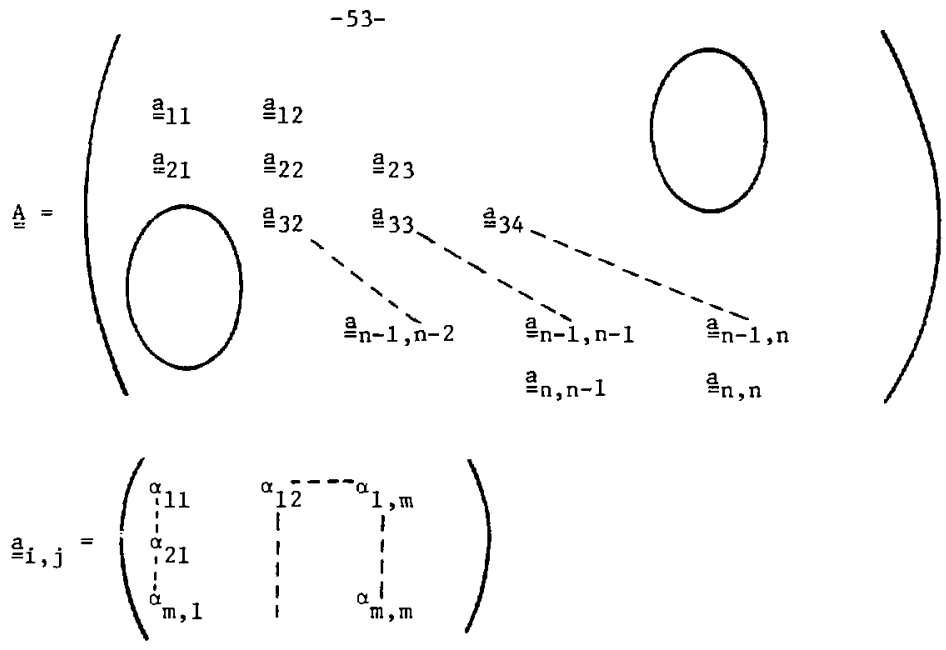

Figure III.1

The block tridiagonal matrix structure found in the onedimensional, three point finite difference equations.

In Section III.4, we develop the algortthm used which takes advantage of the tridiagonal structure to reduce the operations needed for inversion by a factor of $\mathrm{I}^{2}$ to an $\mathrm{I}^{\mathrm{x}} \mathrm{m}^{3}$ dependence. This reduction is accomplished by the need to do full matrix inversions on only one submatrix-sized structure per mesh point. Thus, our 2 minute onedimensional timestep will now take only 17 seconds. For threedimensions, this reduction will not work, as we do not have a block tridiagonal matrix with which to work. The best reduction we can achieve is to treat the matrix $\stackrel{A}{=}$ as a banded matrix. Here the operation count will go as $(\mathrm{m} \times \mathrm{I} \times \mathrm{J} \times \mathrm{K})^{2}, 6268$ hours of computer time. 
We may, however, use alternating direction implicit techniques (ADI) to achieve a reasonable runging time. In this case, we express the three-dimensional problem as three problems in which we advance only one dimension at a time implicitly (see Section III.4 for further details). This technique takes our $64000 \times 64000$ matrix and reduces it to $3 \times 20^{3} \times(8 \times 8)$ matrices yielding a running time of 41 seconds on a CDC-7600 computer. We have assumed here that the problem is linear. Since the MHD equations are nonlinear, we will have to iterate our solutions to assure that we correctly solve the equations. Thus, we may expect running times of 1 to 3 (or more) minutes of 7600 time per timestep.

In the previous discussion, we have not given any criterion for the accuracy of our solution. While it is necessary to have a stable algorithm, it is also necessary to have an accurate one. If we define some factor e, which represents the truncation error of our finite difference equation, as 63

$$
\begin{aligned}
e= & {[\text { differential equation }]_{i j}^{n} } \\
& -[\text { difference equation }]_{i j}^{n}
\end{aligned}
$$

then we wish the error to be expressed as some power of the spatial and temporal mesh sizes.

$$
e=0\left(\Delta t^{A}\right)+0\left(\Delta x^{B}\right)+0\left(\Delta y^{C}\right)+0\left(\Delta z^{D}\right) \text {. }
$$


The exponents A, B, C, and D are 1ntegers greater than zero. We arrive at this accuracy orderlng by using Taylor series expansions for our finite difference equations and then substracting off the original partial differential equation. It is shown in Richtmyer and Morton ${ }^{6}$ that, for the normal centered spatial finite difference approximations on a uniform mesh, the spatial error is of order $\left(\Delta x^{2}\right)$. If we allow $\theta$ in Eq. (III.1.1.6) to be one-half, we get the so-called Crank-Nickolson time weighting which is also of order $\left(\Delta t^{2}\right)$ accurate. Thus, we will have the total error as

$$
e=0\left(\Delta t^{2}\right)+o\left(\Delta x^{2}\right)+o\left(\Delta y^{2}\right)+o\left(\Delta z^{2}\right) .
$$

Our accuracy is only limited by the number of mesh points and by the inaximum timestep size we are willing to use. In the Iimit $\Delta x, \Delta t+0$, the finite difference equation reduces to the partial differential equation.

\section{III.2 The Computational Mesh}

As was stated previously, IMP uses an Eulerian mesh. The values of the state vartable $\underline{u}$, where $\underline{u}=\{\underline{B}, \underline{y}, 0, P\}$, are kept on a set of points in space called mesh points. The values of $\underline{u}$ are to be cell centered quantities. By cell centered we mean that the value of $\underline{u}_{i, j, k}$ is the average value of $u$ in the three-dimensional cell centered at $\left(x_{1}, x_{2}\right.$, $\left.x_{3_{k}}\right)$. The mesh is shown In Fig. III.2 and is examined on a single plane in Fig. III.3. In Fig. III.2, the dots represent the points at which the values of $\underline{u}$ are stored and the triangles represent the interface points at 


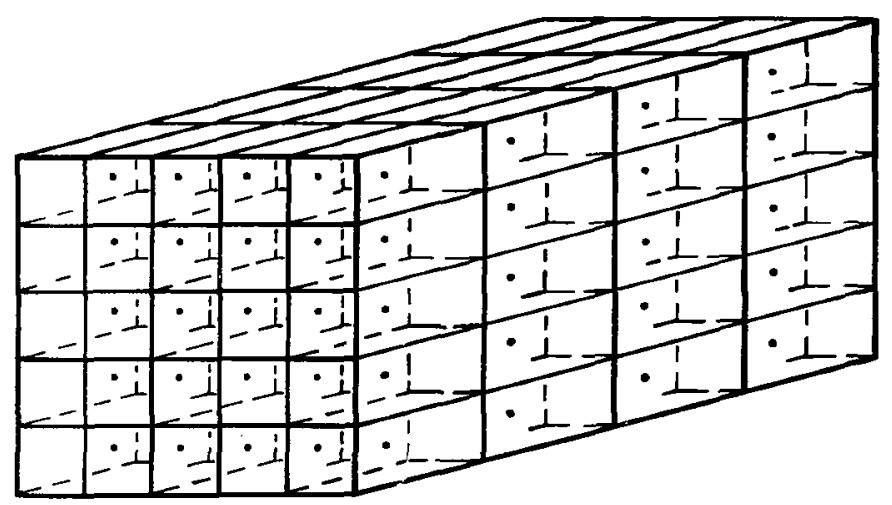

Figure III. 2

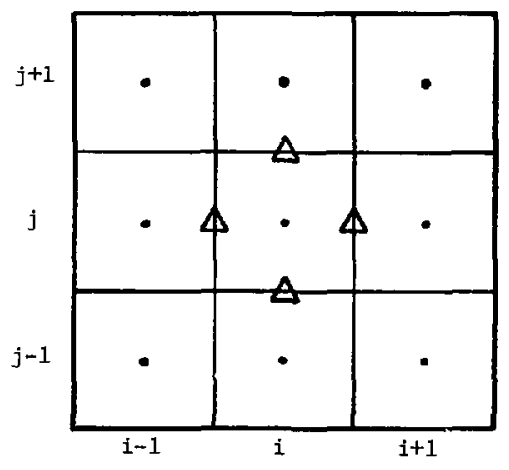

Figure III.3. 
$-57-$

which fluxes and forces are to be evaluated. We define here some quantities and operators that are to be used in the rest of this chapter. We adopt the convention that all unvaried subscripts are deleted $\left(u_{i+1, j, k} \rightarrow_{i+1}\right)$.

First, we define $\dot{n}_{n \pm}$ as the distance between points $x_{n_{i}}$ and $x_{n_{i+1}}$, where $x_{n}=\left(x_{1}, x_{2}, x_{3}\right)$ are some orthogonal curvilinear coordinates,

$$
\begin{aligned}
& i_{1} x_{1+1}-x_{1} \text {, } \\
& \therefore_{1} x_{1}-x_{1}, \\
& i_{2} x_{2}-x_{2}, \\
& z_{-} \quad x_{j}-x_{2 j-1} \text {, } \\
& \therefore_{3} \quad x_{3-1}-x_{3 k} \text {, } \\
& \therefore 3_{3} \quad x_{3}-x_{3-1} .
\end{aligned}
$$

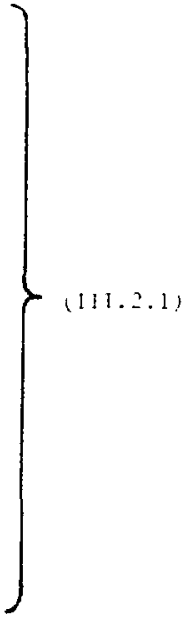

These variable A's allow us to specify a nonuniform mesh. Sext, the distance between interfaces which is called $\therefore$ are given as

$$
\begin{aligned}
& I^{i}-\frac{1}{2}\left(t_{1}+i_{1_{-}}\right)=x_{1_{i+1 / 2}}-x_{1-1 / 2} \\
& 2^{i} \quad \frac{1}{2}\left(i_{2}+i_{2}\right)=x_{2}{ }_{j+1 / 2}-x_{2} x_{j-1 / 2} \\
& 3^{\hat{3}}=\frac{1}{2}\left(\hat{3}_{3_{+}}+\therefore_{3_{-}}\right)=\mathrm{x}_{3_{k+1 / 2}}-\mathrm{x}_{3_{k-1 / 2}}
\end{aligned}
$$


$-58-$

The value of a variable at the interface is expressed by a bar ( - ) operator, such that

$$
\begin{aligned}
& \bar{Q}_{ \pm}-\frac{1}{2}\left(Q_{2+1}+Q_{i}\right) \\
& \bar{Q}_{+} \cdot \frac{1}{2}\left(Q_{j+1}+Q_{j}\right) \\
& \bar{Q}_{+}-\frac{1}{2}\left(Q_{k+1}+Q_{k}\right)
\end{aligned}
$$

The "operator gives the difference across an interface:

$$
\begin{aligned}
& l_{+}(F) \frac{1}{1_{+}}\left(F_{i+}-F_{i}\right) \\
& 1_{-}(F) \frac{1}{a_{-}}\left(F_{i}-F_{i-1}\right) \\
& 2_{+}(F) \frac{1}{2_{+}}\left(F_{j+1}-F_{j}\right) \\
& 2_{-}(F)=\frac{1}{\Delta_{2}}\left(F_{j}-F_{j-1}\right) \\
& 3_{+}(F)=\frac{1}{a_{3}}\left(F_{k+1}-F_{k}\right) \\
& 3_{-}(F)=\frac{1}{\Delta_{3}}\left(F_{k}-F_{k-1}\right)
\end{aligned}
$$




\section{III.3. Spatial Finite Differencing}

The MHD Eqs. (Ii.5.13) contain two basic types of terms. The first Lype are terms that arise from the divergence of fluxes in the conservation law equations. This includes the pseudovector conservation law of the magnetic flux equation. In the expanded equations, all terms of the form $\frac{\partial}{\partial x_{1}}(\propto \phi)$, where $\phi$ is any general factcr, all called FIJUX ierms. For example, ; may be $\frac{\partial \psi}{\partial x_{2}}$. Thus both plane second derivatives and mixed second derivatives are subsets of the Flux terms. The second type of term that appears in the (II.5.13) has the form $\alpha \frac{\partial u}{\partial x_{1}}$ where $\alpha$ is sume function of $u$. For example, in the momentum equation, we see terms of the form $B \frac{B}{3 x_{1}}$ and $\frac{\partial p}{\partial x_{1}}$ which arise $f$ com the $J \times \underline{B}$ forces and the pressure gradient forces. Terms of this form also appear in the energy equation (i.e., $v \frac{3 P}{3 x_{1}}$ and $\left.\frac{n}{\mathrm{~S}} \frac{\partial \mathrm{B}}{j \mathrm{x}_{1}} \frac{\dot{b}}{\partial \mathrm{x}_{2}}\right)$. These are categorized is FORCE terms. It is to be stressed that the titles FLUX and FORCE are used to categorize the form of the ierms to be differenced and not co label their physical significance.

When we :t up our spatial finite differencing, we are representing: system of equations that describo changes to our cell-centered quantitirs due to fluxes into and out of the cells and forces applied to the cells. Both these fluxes and :orce: must be calculated at the cell interfaces (see Fig. III.3, triangles). Brised on this scheme, we use the following techniques for generating the finite approximations.

\section{III.3.1 Flux Term Jifferencing}

The FLUX terms measure flow into or out of the computational cell. Therefore, these terms must be evaluated at the cell boundaries. Thus, 
the finite difference form is

$$
\frac{\partial}{\partial x_{1}}(\alpha F)+\frac{\bar{\alpha}_{+} \bar{F}_{+}-\bar{\alpha}_{-} \bar{F}_{-}}{1^{\Delta}}
$$

where we use the definitions of Section III.2 for $\Delta^{\Delta}$ and the - (bar) operator.

$$
\begin{aligned}
& \text { When } F \text { represents a derivative }\left(F=\frac{\partial G}{\partial x_{1}}\right) \text {, we use } \\
& \frac{\partial}{\partial x_{1}}\left(a \frac{\partial G}{\partial x_{1}}\right)=\frac{\bar{a}_{+} \delta_{1}(G)-\bar{\alpha}_{-} \delta_{1} \text { (G) }}{1 \Delta}
\end{aligned}
$$

The \& operator (Section III.2) Is the difference across the interface, evaluated at the interface, so we have a consistent approximation. If we assume that we are on a uniform mesh and $\alpha$ is a constant, the definitions given in Egs. III.3.1.1 and III.3.1.2 reduce to the normal first and second derivative finite $d f f$ ference approximations:

$$
\frac{\partial}{\partial x_{1}}(\alpha F)=\alpha \frac{F_{i+1}-F_{i-1}}{2 \Delta x}
$$

and

$$
-\frac{\partial}{\partial x_{1}}\left(\alpha \frac{\partial G}{\partial x_{1}}\right)=\approx \frac{G_{i+1}-2 G_{i}+G_{i-1}}{(\Delta x)^{2}}
$$

In the case of mixed derivatives,

$$
F=\frac{\partial G}{\partial x_{2}}
$$


we take the derivative in the $x_{2}$ direction at points $A_{+}, A_{-}, B_{+}$and $B_{-}$ in Fig. III.4 and average them to arrive at a value of $\bar{F}$ at a cell face $(x)$. This gives

$$
\frac{\partial}{\partial x_{I}}\left(\alpha \frac{\partial G}{\partial x_{2}}\right)=\frac{\bar{\alpha}_{+}\left(\frac{\partial G}{\partial x_{2}}\right)-\bar{\alpha}_{-}\left(\frac{\partial G}{\partial x_{2}}\right)}{1^{\Delta}}=
$$

\begin{tabular}{|c|c|c|}
\hline $\begin{array}{c}i-1, j+1 \\
0\end{array}$ & $\begin{array}{cc} & i, j+1 \\
A_{+} & 0\end{array}$ & $\begin{aligned} & i+1, j+1 \\
& 0 \\
B_{+} & \end{aligned}$ \\
\hline $\begin{array}{c}i-1, j \\
0\end{array}$ & A & $\begin{array}{c}\mathbf{i}+1, \mathbf{j} \\
\circ\end{array}$ \\
\hline $\begin{array}{c}i-1, j-1 \\
0\end{array}$ & $\begin{array}{c}i, j-1 \\
0\end{array}$ & $\begin{array}{c}i-1, j-1 \\
0\end{array}$ \\
\hline 0 & 0 & 0 \\
\hline
\end{tabular}

Figure III.4. Mixed Derivative Calculation

where

$$
\begin{aligned}
\left(\frac{\partial \bar{G}}{\partial \bar{x}_{2}}\right)_{+} & =\frac{1}{4}\left(\left[G_{i+1, j+1}-G_{i+1, j}+G_{i, j+1}-G_{i, j}\right] / \Delta_{2 \dashv}\right. \\
& \left.+\left[G_{i+1, j}-G_{i+1, j-1}+G_{i, j}-G_{i, j-1}\right] / \Delta_{2}\right)
\end{aligned}
$$

Notice that terms are evaluated at the center of the interfaces in all cases . 
From the preceding, it can be seen that the FLUX terms are antisymmetric. That is, the fIux $\underline{E}+$ at the ith grid point becomes minus the flux $E$ - in the $i+I^{\prime}$ 's grid point. 'Therefore, the flux $F+{ }_{i}$ is calculated when we are at the ith grid point and is reused as $-F$ at the next grid point. This double use of $\underline{F}$ allows us to avoid extra calculations and also the extra FORTRAN required for the calculations. This is one less chance for errors to come fnto the code.

\section{III.3.2 Force Term Differencing}

The FORCE terms measure the forces applied on the edges of the computational ce1l. As such, we define them as the average of the forces applied on each cell face. The finite difference form is

$$
\alpha \frac{\partial F}{\partial x_{1}}=\frac{1}{2}\left(\bar{a}_{+} \delta_{1_{+}}(F)+\bar{\alpha}_{-} \delta_{1_{-}}(F)\right)
$$

where the $\delta_{1_{+}}$operator has been defined In Section III.2.

Products of derivatives arise from the $n j^{2}$ terms in the internal energy equation. This product can be put in the form of

$$
\begin{aligned}
& \alpha \frac{\partial F}{\partial x_{1}}+\frac{\partial G}{\partial x_{2}} \frac{\partial F}{\partial x_{1}}= \\
& \quad \frac{1}{2}\left[\left(\frac{\partial G}{\partial x_{2}}\right)_{i+1 / 2} \delta_{1_{+}}(F)+\left(\frac{\partial G}{\partial x_{2}}\right)_{i-1 / 2} \delta_{1-}(F)\right]
\end{aligned}
$$

The term $\left(\frac{\partial G}{\partial x_{2}}\right)_{i \pm 1 / 2}$ is evaluated in an identical fashion to $\left(\frac{\partial G}{\partial x_{2}}\right)_{j .1 / 2}$ in the FLUX terms (see Eq. III.3.6.3). 
The main problem with the mixed derivative and product of derivative terms comes in attempting to correctly time-ronter them. This will be addressed in the next section.

As the FLUX terms are antisymmetric, the FORCE terms are symmetric. In this case $\underline{F}+$ at the ith grid point is equal to $\underline{F}$ - at the $i+l^{\prime}$ st grid point. Once again, this property is taken advantage of in the IMP code.

\section{III.4 Temporal Finite Differencing}

As was noted in Chapter I, IMP uses the Douglas-Gunn ${ }^{5}$ algorithm (DGA) for controlling its time advance. The DGA is an extension of normal alternating direction implicit (ADI) techniques to three dimensions. These ADI techniques allow for the solution of the large 3-n matrix problem in reasonable amounts of computer time.

We express the set of MHD equations as the vector equation

$$
\frac{\partial \underline{U}(\underline{u})}{\partial t}+\underline{F}(\underline{u})+\underline{G}(\underline{u})+\underline{H}(\underline{u})=0
$$

where $\underline{U}$ is the set $\{\underline{B}, p \underline{v}, p, P\}, \underline{u}$ is the set $\{\underline{B}, \underline{v}, p, P\}$ and $\underline{F}(\underline{u}), \underline{G}(\underline{u})$ and $\underline{H}(\underline{u})$ are given in Eqs. III.1.2, III.1.3, and III.I.4 respectively. Then the time centering DGA technique yields the three step approach:

$$
\frac{\underline{\mathrm{U}}^{\mathrm{n}^{*}}-\underline{\mathrm{U}}^{\mathrm{n}}}{\Delta \mathrm{t}}+\frac{\mathrm{I}}{2}\left[\underline{\mathrm{F}}\left(\underline{\mathrm{u}}^{\mathrm{n}}\right)+\underline{\mathrm{F}}\left(\underline{\mathrm{u}}^{\mathrm{n}}\right)\right]+\underline{\mathrm{G}}\left(\underline{\mathrm{u}}^{\mathrm{n}}\right)+\underline{\mathrm{H}}\left(\underline{\mathrm{u}}^{\mathrm{n}}\right)=0
$$




$$
\begin{aligned}
& -64- \\
& \frac{\underline{\mathrm{U}}^{\mathrm{n} * \star}-\underline{\mathrm{U}}^{\mathrm{n}}}{\Delta \mathrm{t}}+\frac{1}{2}\left[\underline{\mathrm{F}}\left(\underline{\mathrm{u}}^{\mathrm{n}^{*}}\right)+\underline{\mathrm{F}}\left(\underline{\mathrm{u}}^{\mathrm{n}}\right)+\underline{\mathrm{G}}\left(\underline{\mathrm{u}}^{\mathrm{n}}\right)+\underline{\mathrm{G}}\left(\underline{\mathrm{u}}^{\mathrm{n}}\right)\right] \\
& +\underline{H}\left(\underline{u}^{n}\right)=0 \\
& \frac{\underline{U}^{n+1}-\underline{U}^{n}}{\Delta t}+\frac{1}{2}\left[\underline{F}\left(\underline{u}^{n \star}\right)+\underline{F}\left(\underline{u}^{n}\right)+\underline{G}\left(\underline{u}^{n * \star}\right)+\underline{G}\left(\underline{u}^{n}\right)\right. \\
& \left.+\underline{H}\left(\underline{u}^{n+1}\right)+\underline{\underline{y}}\left(\underline{u}^{n}\right)\right]=0
\end{aligned}
$$

Equations III.4.2 are combined to yield the final set

$$
\begin{aligned}
& \frac{\underline{U}^{n *}-\underline{U}^{n}}{\Delta t}+\frac{1}{2}\left(\underline{F}\left(\underline{u}^{n \star}\right)+\underline{F}\left(\underline{u}^{n}\right)\right)+\underline{G}\left(\underline{u}^{n}\right)+\underline{H}\left(\underline{u}^{n}\right)=0 \\
& \frac{\underline{U}^{n \star *}-\underline{u}^{n *}}{\Delta t}+\frac{1}{2}\left(\underline{G}\left(\underline{u}^{n \star \star}\right)-\underline{G}\left(\underline{u}^{n}\right)\right)=0 \\
& \left.\frac{\underline{\mathrm{U}}^{\mathrm{n}+1}-\underline{\mathrm{U}}^{\mathrm{n} * *}}{\Delta \mathrm{t}}+\frac{1}{2}\left(\underline{\mathrm{H}}^{\left(\underline{u}^{\mathrm{n}+1}\right.}\right)-\underline{\mathrm{H}}\left(\underline{\mathrm{u}}^{\mathrm{n}}\right)\right)=0
\end{aligned}
$$

The set of equations III.4.3 describe the time afvancement scheme employed in IMP. Equation III.4.3.a may be thought of as a predictor step while III.4.3.b and III.4.3.c are corrector steps. By assuming particular forms for the $\underline{F}, \underline{G}$ and $\underline{H}$ in III.4.2, we can arrive at linear stability criterion for DGA. If we let $\underline{F}, \underline{G}$ and $\underline{H}$ be diffusion or advection terms, then $D G A$ is linearly unconditionally stable. ${ }^{6}$ The linear unconditional stability Is the reason for choosing an implicit time advancement scheme. We are able to let the relevant physics determine our timestep rather than being limited by numeric considerations. 
The $(1,2)$ and $(1,3)$ symmetry pointed out in Section III.1 allows us to use identical paths to calculate the $E$ function in III.4.3.a, the $\underline{G}$ function in III.4.3.b and the $\underline{H}$ function in III.4.3.c. Therefore, except for the sign change in the explicit function evaluation in the second and third equations and the cross $I$ ine and cross plane $\underline{G}$ and $\underline{H}$ functions in the Eirst equation, we can use identical code for all three subtimesteps.

The accuracy of the algorithm is found by eliminating $\underline{u}^{n *}$ and $\underline{u}^{n * *}$ frot the third equation of III.4.2. By defining the following relationships,

$$
\begin{aligned}
& \underline{\Delta u}=\underline{u}^{n+1}-\underline{u}^{n} \\
& \underline{u}^{n+l / 2} \equiv \frac{1}{2}\left(\underline{u}^{n+1}+\underline{u}^{n}\right)
\end{aligned}
$$

we express $\underline{u}^{n *}$ and $\underline{u}^{n * *}$ as

$$
\underline{u}^{n *}=\underline{u}^{n+1}+\frac{\Delta t}{2}\left\{(\underline{G}(\Delta \underline{u})+\underline{H}(\Delta \underline{u}))+\frac{\Delta t}{2} \underline{G}(\underline{H}(\underline{\Delta} \underline{u}))\right\}
$$

and

$$
\underline{u}^{n * *}=\underline{u}^{n+1}+\frac{\Delta t}{2} \underline{H}(\Delta \underline{u}) .
$$


Then we have

$$
\begin{gathered}
\frac{\underline{u}^{n+1}-\underline{u}^{n}}{\Delta t}+\underline{F}\left(\underline{u}^{n+1 / 2}\right)+\underline{G}\left(\underline{u}^{n+1 / 2}\right)+\underline{H}\left(\underline{u}^{n+1 / 2}\right) \\
+\frac{\Delta L}{4}\{\underline{F}(\underline{G}(\Delta \underline{u})+\underline{H}(\underline{\Delta u}))+\underline{G}(\underline{H}(\Delta \underline{u}))\} \\
+\frac{\Delta L^{2}}{8} \underline{F}(\underline{G}(\underline{H}(\underline{\Delta})))=0 .
\end{gathered}
$$

The first term is the ffnite difference of $\underline{u}$ time centered at $\underline{u}^{n+1 / 2}$. The secund term is a Crank-Nicholson weighted term which is correct to order $\therefore t^{2}$. The third term is of order $\Delta t^{2}$ and the fourth of order $\Delta t^{3}$. Hence the overall time advance algorithm's accuracy is of order $\Delta t^{2}$. Since the spatial finite differences that make up $\underline{F}, \underline{G}$, and $\underline{H}$ are of order $\Delta x_{1}^{2}, \Delta x_{2}^{2}$ and $i x_{3}^{2}$ on a uniform mesh, the overall accuracy for our FDE's (neglecting the mixed derivative and product of derivative terms) is

$$
\begin{gathered}
e=0\left(\Delta t^{2}\right)+o\left(\Delta x_{1}^{2}\right)+o\left(\Delta x_{2}^{2}\right)+0\left(\Delta x_{3}^{2}\right)+ \\
o\left(\Delta x_{1} \Delta x_{2}\right)+o\left(\Delta x \Delta x_{3}\right)+o\left(\Delta x_{2} \Delta x_{3}\right)
\end{gathered}
$$

The forms of $\underline{F}, \underline{G}$ and $\underline{H}$ in Eq. (III,1,1) are well defined for all but the mixed and product derivatives. Here, we are faced with trying to get advanced time values on rows of the mesh not yet computed. Killeen and Marx ${ }^{64}$ split the time depondence of the cross-line (e.g., $x_{2}$ derivatives on an $x_{1}$ sweep) derivatives to take the $\delta_{2}$ terms, assuming the calculation was proceeding up the plane, implicttly and the $\delta_{2}$ terms explicitly. One would 
then sweep downward on the next sweep to obtain time centering on these terms. One drawback of this method is that the mixed derivative terms are not being calculated in a conservative manner. This may be seen by looking at the term across two rows of the mesh. We will take $\frac{\partial}{\partial x_{1}}\left(a \frac{\partial F}{\partial x_{2}}\right)$ on lines $j$ and $j+1$. On line $j$ we have, looking at the $1+1 / 2$ interface only,

$$
\begin{aligned}
& \left.\frac{\partial}{\partial x_{1}}\left(a \frac{\partial F}{\partial x_{2}}\right)^{n \neq}\right|_{x_{1}=x_{1}}, x_{2}=x_{2}= \\
& \vec{v}_{+, 2}\left[\delta_{2}\left(F^{n}\right)+\delta_{2}, i+1\left(F^{n}\right)\right] \\
& +\bar{a}_{1_{+}, 2}\left[\delta_{2},\left(^{n^{*}}\right)+\delta_{2-, i+1}\left(F^{n *}\right)\right]
\end{aligned}
$$

and

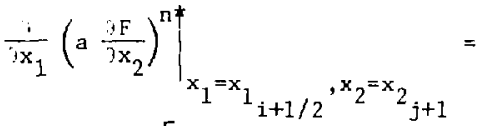

$$
\begin{aligned}
& \bar{a}_{1+}, 2_{+}\left[z_{++, i}\left(F^{n}\right)+i_{2} z_{++, i+1}^{j+1}\left(F^{n}\right)\right]
\end{aligned}
$$

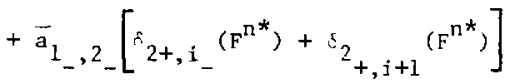

Here, we can see that the $\delta_{2}$ terms are not evaluated at the same time level on successive rows and so, like terms cannot cancel. The lack of cancellation of interior terns means that the equations have not been conservarively differenced (see, e.g., Potter ${ }^{7}$ pp 61-63). 
Since we will be required to iterate due to the nonlinearity of the equations (see Section III.7.2), another approach is to evaluate these terms at the last iteration level. That is, we approximate the terms as

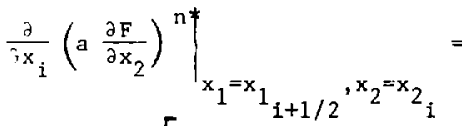

$$
\begin{aligned}
& \left.\left.\vec{a}_{+}, 2+\delta_{2+, i}\left({ }^{\ell-1} F^{n^{*}}\right)+\delta_{2}{ }^{\ell-1}{ }^{\ell-1} \mathrm{~F}^{*}\right)\right]
\end{aligned}
$$

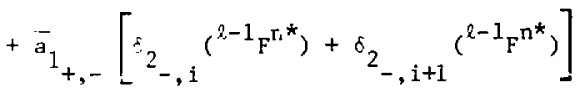

where the left superscript \& refers to the iteration number (see Section III. 5 for a description of the iteration procedure). In this case, the derivative is calculated in a conservative manner since there is no time mixing of terms. The problem that arises here is that we are required to iterate the mesh as a whole to keep everything at the same iteration level. This is wasteful since, in general, the number of iterations required differs from row to row and we are forced to use the maximum number for the whole mesh.

Finally, we can treat the mixed and product terms fully explicitly. Tlis lowers the accuracy to order $\Delta t$ for these terms and introduces a Courant limit $\because$ the time step size. A variation on this explicit method for the product terms that gives some time centering is

$$
\left(\frac{\partial F}{\partial x_{1}} \frac{\partial G}{\partial x_{2}}\right)^{n *}=\frac{1}{2}\left(\frac{\partial F^{n}}{\partial x_{1}} \frac{\partial G^{n^{*}}}{\partial x_{2}}+\frac{\partial F^{n *}}{\partial x_{1}} \frac{\partial G^{n}}{\partial x_{2}}\right)
$$


All of the above methods can be implemented in IMP but in general we use fully explicit mixed derivatives and use Eq. (III.4.13) for time centering the product of derivative terms. This choice, coupled with time step controls (see Section III.8), has run at hundreds of times the Courant limit for some problems with no ill effects.

\section{III.5 Linearization of the Finite Difference Equations}

Applying the resilts of the preceding sections to the MHD equations yields a set of nonlinear algebraic equations of the form

$$
\begin{aligned}
& \underline{T}\left(\underline{u}^{n *}\right)-\underline{T}\left(\underline{u}^{n}\right)+\frac{\Delta t}{2}\left[\underline{F}\left(\underline{u}^{n \star}\right)+\underline{F}\left(\underline{u}^{n}\right)\right] \\
& +A \cdot\left[G\left(\underline{u}^{n}\right)+H\left(\underline{u}^{n}\right)\right]=0
\end{aligned}
$$

where $\underline{u}=\{\underline{B}, \underline{v}, n, P\}$ and $\underline{T}(\underline{u})=\{\underline{B}, \rho \underline{v}, 0, p\}$. The term $r \underline{v}$ in $\underline{T}$ is an example of a nonlinear term. Since, neglecting mixed derivative terms, our differencing is of order $\left(\Delta x^{2}, \Delta t^{2}\right)$ we would like to linearize the equations to the same accuracy in $\Delta t$. We can use a Taylor series expansion in $t$ ime to yield as a linearization formula for any nonlinear term $f$,

$$
\underline{f}\left(t=n^{*}\right)=\underline{f}(t=n)+\underline{J} \cdot \frac{\partial \underline{u}}{\partial t} \Delta t
$$

where

$$
J-\frac{\partial \underline{f}}{\partial \underline{u}^{n *}}
$$


which is the Jacobian of the term. This procedure is carried one step further by recognizing that this can be expressed as the first step of a generalized Newton-Raphson iteration. By letting the set of nonlinear algebraic equations (III.5.1) be written

$$
F=n,
$$

We van use III.5.2 and iet $\underline{f}\left(t=\Omega^{*}\right)=\underline{F}=0$. We then have

$$
\eta=-1 F+\cdot-\frac{1}{y^{n^{\star}}} \cdot \frac{\frac{7 \underline{u}^{n^{\star}}}{i t}}{i t}
$$

$a r$

$$
u^{n^{*}}=i_{\underline{u}}^{-1} n^{*}-\left(i-1 \underline{J}^{n *}\right)^{-1} \cdot{ }^{i-1} \underline{F}^{n^{*}}
$$

where. is the iteration index. Letting ${ }^{0} \underline{u}^{\mathrm{n}^{\star}}$ take on the value of $\underline{u}^{n}$ gives us the first step which corresponds to equation III.5.2. To see the form of Eq. (1II.5.5) that is actually to be iterated, we multiply through by ${ }^{-1} \stackrel{J}{=}$ vielding (where ${ }^{\star}{ }^{*}$ superscripts has been suppressed)

$$
-1_{\underline{J}} \cdot{ }^{\imath} \underline{\underline{u}}={ }^{i-1} \underline{J} \cdot i-1 \underline{u}-{ }^{i-1} \underline{F} .
$$

When the finite difference approximations are substituted into Eq. III.5.6 for $\underline{F}$, the matrix takes on a block tridiagonal form. This form is 
represented by

$$
-A_{=j} \cdot \underline{u}_{i+1}+\underline{B}_{i} \cdot \underline{u}_{i}-\underline{C}_{i} \cdot \underline{u}_{i-1}=\underline{D}_{1} \text {. }
$$

In order to obtain the values of $\stackrel{A}{\underline{B}}, \underline{\underline{C}}$, and $\underline{\mathrm{D}}$, we must consider the form of the MHD equations $\underline{F}(\underline{u})=0$. We express $\underline{F}$ in two parts, the explicit terms $\underline{F}_{e}$ which depend only on $\underline{u}^{n}$ and the Implicit terms $\underline{F}_{i}$ whirh depend on $\underline{u}^{n k}$ and possibly on $\underline{u}^{n}$. Thus, we have

$$
\underline{F}(\underline{u})=\underline{F}_{e}\left(\underline{u}^{n}\right)+\underline{F}_{1}\left(\underline{u}^{n^{*}}, \underline{u}^{n}\right) .
$$

Since the Jacohian $\leqq$ measures only the $\underline{u}^{n *}$ dependence, we can inmediatelv group all the explicit terms in the right hand side D vector of Eu.

(III.5.7). Next we look at some point $j$ on the mesh. At this puint, the equation to be solved is

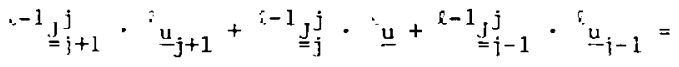

$$
\begin{aligned}
& \stackrel{-1}{J}_{j+1}^{j} \cdot{ }^{\ell-1} \underline{u}_{j+1}+{ }^{i-1} \underline{J}_{j}^{j} \cdot{ }^{\ell-1} \underline{u}_{j} \\
& +{ }^{i-1} \underline{J}_{j-1} \cdot{ }^{s-1} \underline{u}_{j-1}-\underline{F}_{j}
\end{aligned}
$$

where

$$
{ }_{i}-1 j_{j+1}^{j}-\frac{j^{i-1} F_{-i}}{\partial u_{j+1}^{n^{\star}}},
$$




$$
\begin{aligned}
& \cdot-1 \frac{j}{=j} \cdot \frac{i^{i-1} \underline{F}_{j}}{a_{-u^{n}}^{n^{*}}}, \\
& { }^{-1} \underbrace{}_{i-1} \frac{{ }^{-1} \underline{F}_{i} j}{u_{i-1}^{n^{\star}}}
\end{aligned}
$$

ans: ${ }^{-1} F_{i}$ is the implicit part of $\underline{F}$ at the $j-t h$ gria ioint. This equation mit: Further hroken down bv using the propetties of the spatial finite

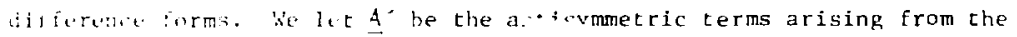
implis it lX terms and $S^{-}$be the summetric Lerms arssing from the implicit

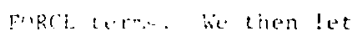

$$
F_{i}=4_{i+1} / 2-4_{i-1 / 2}+s_{i+1 / 2}+s_{i-1 / 2}
$$

Ce roberine the 'acotian. (Eq. III.5.10) to he

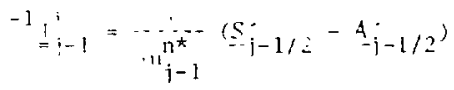

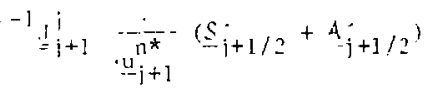

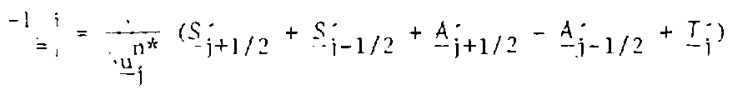

where $T_{j}$ ire the Lerms arising from the time derivatives ir F. (e.t., $\therefore, \frac{\ddots v}{\cdot t}$, etc ). Next, we define the functions $\stackrel{A}{=}$, and $I$ such that

$$
\stackrel{s}{j}_{j}^{+} \quad \frac{s_{j+1}^{-2}}{\underline{u}_{j+1 / 2}^{n^{*}}}
$$


$S_{j}-\frac{3 S_{j+1 / 2}}{\underline{u}_{j}^{n^{*}}}$;

$A_{1}^{+} \quad \frac{3 A_{j+1 / 2}}{a_{j+1 / 2}^{n^{\star}}}$

$\stackrel{A}{=} j \quad \frac{A j+1 / 2}{\cdot u^{n^{*}}}$;

( I I T.5.13)

$\stackrel{T}{=} \quad \frac{T^{\cdot}}{n^{\dot{*}}}$.

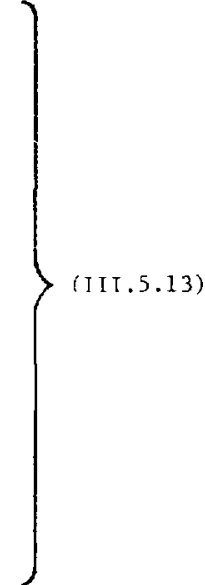

Given the above functions. we can now write down the forms of $\stackrel{A}{=} \stackrel{\text { E }}{=}$, and 1) of equation (III,5.7) that are actually used in LYP. We have

$\stackrel{A}{=} \mathrm{j}=-\left(S_{j}^{+}+\stackrel{A}{=}_{j}^{+}\right)$

$(I ! 5.14 .3)$

$\stackrel{C}{=}_{j}=-\left(S_{j}-1-\stackrel{S}{:}_{j-1}\right)$

('II.5.14.6)

$\stackrel{B}{=}_{j}=\underline{\underline{I}}_{j}+\stackrel{S}{\underline{S}}_{j}+\stackrel{S}{\underline{S}}_{j-1}^{+}+\stackrel{A}{=}_{j}-\stackrel{A}{=}_{j-1}^{+}$

(III.5.14.c)

and

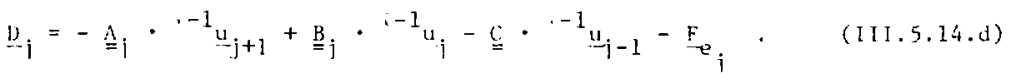


Using the form given in equations III.5.14 allows us to only calculate the Jacobians on the forward cell interface and then reuse the values from the previous cell for the hackward interface.

The $\stackrel{A}{=}^{+}, \stackrel{A}{=}, \underline{S}^{+}$and $\underline{S}$ matrix elements must now be getierated. They are determined by the analytic dertvatives given in Eq. III.5.13 and are $8 \times 8$ matrices. We, therefore, must evaluate $5 \times 64$ matrix elements, many of which have complicated functional dependences on $\underline{u}^{n *}$, (e.g., we gentrally use the Spltzor 65 resistivity $\left.n \propto T^{-3 / 2} \propto\left(\frac{P}{\rho}\right)^{-3 / 2}\right)$. Any time we wish to try a different difference technique, we must regenerate the elements of the Jacobian matrix. To simplify this procedure, the FDE's were programmed on REDUCE, ${ }^{46}$ an algebraic processor running on the MFE-PDP10. After defining the set of MHD equations and various fintte difference techniques to REDUCE, and then programming it to take a Jacobian, the pain of producing the elements of the Jacobian Is mostly alleviated. REDUCE can write the matrix elements in the form of a FORTRAN subroutine to further ease the process. The use of the REDUCE processor has allowed us the trial of many different finite difference techniques without spending weeks dertving the various matrix elements and then attempting to enter them into the computer correctly. A listing of the REDUCE g: rerator code is given in Appendix B. Once agaln we point out that, due to the $(1,2)$ and $(1,3)$ symmetry of the $\underline{E}$, $\underline{G}$ and $\underline{H}$ functions, the Jacobians need only be generated for the first subtimestep. They are then reused for the second and third subtimestep. 
Having calculated the Jacobian matrix elements, we now have a simple formula (Eq. III,5.6) to follow by which we iterate our mesh to some convergence criterion $\left(\underline{\varepsilon} \leq\left(\underline{\underline{u}}-\ell^{\ell-1} \underline{u}\right) /^{\ell-1} \underline{u}\right)$. The reason we do the iterations and not just stop at one pass as do Brilly and McDonald, ${ }^{66}$ which is formally correct to $O(\Delta t)^{2}$, is that, when we simplified Eq. (III.4.2) to get the set Eq. (III.4.3), we assumed that previously calculated functions cancelled. For example, we have assumed that $\underline{F}\left(\underline{u}^{n *}\right)$ in Eq. (III.4.2.a) was equal to $\left.\underline{\mathrm{F}}^{\left(\mathrm{u}^{\mathrm{*}}\right.}{ }^{*}\right)$ in Eq. (III.4.2.b). This cancellation will only be true if we actually iterate the equations to convergence.

\section{IJI.6 Solution of Difference Equations}

As was previously stated, we want to solve a system of I equations whose form is block-tridiagonal. This system can be represented by

$$
-\stackrel{A}{=}_{i} \cdot \underline{u}_{i+1}+\underline{B}_{i} \cdot \underline{u}_{i}-\underline{C}_{i} \cdot \underline{u}_{i-1}=\underline{D}_{i} \quad, \mathbf{i}=2,3, \ldots, I-1 \quad \text { (III.6.I) }
$$

We will assume that the boundary conditions can be expressed in the form

$$
\stackrel{G}{=}_{1} \cdot \underline{\mathrm{u}}_{1}=\stackrel{\mathrm{H}}{=}_{1} \cdot \underline{\mathrm{u}}_{2}+\underline{\mathrm{J}}_{1}
$$

and

$$
\underline{G}_{\mathrm{I}} \cdot \underline{\underline{\mathrm{u}}}_{\mathrm{I}}=\underline{\underline{\mathrm{H}}}_{\mathrm{I}} \cdot \underline{\mathrm{u}}_{\mathrm{I}-1}+\underline{\mathrm{J}}_{\mathrm{I}} \text {. }
$$

Taking the relation

$$
\underline{u}_{i}=\underline{E}_{i} \cdot \underline{u}_{i+1}+\underline{F}_{i} \quad, i=1,2, \ldots, I-1
$$


and substituting for $\underline{u}_{i-1}$ in Eq. (III.6.1), we get the recurrence relations for $\underline{E}_{\mathbf{i}}$ and $\underline{F}_{\mathbf{i}}$ as

$$
\underline{E}_{1}=\left(\underline{B}_{i}-\underline{C}_{i} \cdot \underline{E}_{i-1}\right)^{-1} \cdot \stackrel{A}{=}_{i}
$$

and

$$
\underline{F}_{i}=\left(B_{i}-\underline{C}_{i} \cdot \underline{E}_{i-1}\right)^{-1} \cdot\left(\underline{D}_{i}+\underline{C}_{1} \cdot \underline{F}_{i-1}\right)
$$

for $i=2,3, \ldots, I-1$. We have, then, a straightforward algorithm for the solution of Eq. (III,6.1), provided we can use the boundary conditions IJI.6.2 to give us values for $\underline{E}_{1}, \underline{F}_{1}$ and $\underline{u}_{I}$.

By writing Eq. (III.6.2.b) in the form of Eq. (III.6.3), we see by inspection that

$$
\underline{E}_{1}=\underline{G}_{1}^{-1} \cdot \stackrel{H}{=}_{1}
$$

and

$$
\underline{F}_{-1}=\underline{G}^{-1} \cdot \underline{J}_{1}
$$

Next, we use Eq. (III.6.3) to substitute for $\underline{\mathrm{I}}_{\mathrm{I} 1}$ in Eq. (III.6.2.b). This yle1ds

$$
\underline{u}_{I}=\left(\underline{\underline{G}}_{I}-\underline{\underline{H}}_{I} \cdot \underline{\underline{E}}_{I-1}\right)^{-1} \cdot\left(\underline{J}_{I}+\underline{\underline{H}}_{I} \cdot \underline{\underline{F}}_{I-1}\right) \cdot
$$

Our solution method is now clear. We use $\mathrm{Eq}$. (III.6.5) to get $\mathrm{E}_{1}$ and $\underline{E}_{1}$; sweep from $i=2,3, \ldots, I-1$ solving $E q$. (III.6.4) for $\underline{E}_{i}$ and $\underline{F}_{i}$; use 
Eq. (III.6.6) to get $\underline{u}_{I}$ and then backsubstitute using Eq. (III.6.3). The most costly part of the algorithm is inverting the matrix $(\underline{\underline{B}}-\underset{\underline{C}}{\underline{E}}$ ) at each mesh point. For the MHD equations used in $I P$, this is an $8 \times 8$ matrix which requires 450 microseconds (or 345 microseconds for the reduced $7 \times 7$ set) to produce the inverse on a CDC-7600 using fully optimized machine language LU decomposition subroutines. 67

The above solution algorithm will work for any boundary conditions that can be cast in the form of Eq. (III.6.2). Periodic boundary conditions, on the other hand, have the relations that $\underline{u}$ and $\frac{\partial \underline{u}}{\partial n}$ (where $n$ is the direction of the line) are equal at the erds of pe.iodic meshes. This is implemented by assuming that

$$
\begin{aligned}
& \underline{u}_{1}=\underline{u}_{I+1} \\
& \underline{u}_{2}=\underline{u}_{I+2}
\end{aligned}
$$

and that the point $i=3 / 2$ is where the periodic boundary occurs. The points $I+1$ and $I+2$ do not actually exist as they are redundant. Here, we first assume a recurrence relation

$$
\underline{u}_{i}=\underline{E}_{i} \cdot \underline{u}_{i+1}+\underline{E}_{i}+\underline{s}_{i} \cdot \underline{u}_{1}
$$

$\mathbf{i}=2,3, \ldots I-1$. The inclusion of the $\underline{S}_{\mathbf{i}} \underline{u}_{\mathbf{u}}$ term takes into account the fact that now the matrix has the cridiagonal form plus elements in the upper right and lower left corners (see Fig. III.5). Eliminating $\underline{u}_{i-1}$ from Eq. (III.6.1) now gives us the relations 

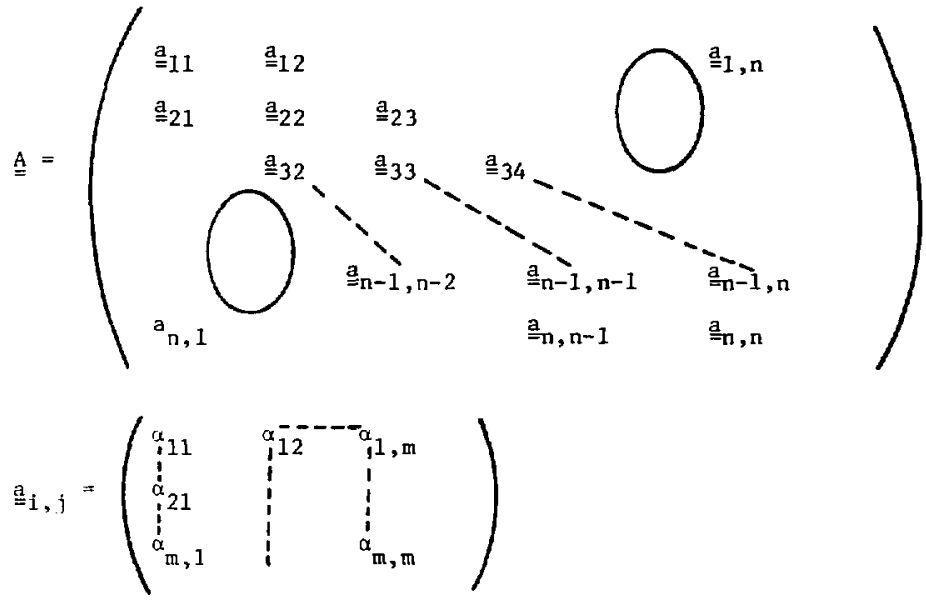

Figure III.5

The block tridiagonal matrix structure found in the one-dimensional, three noint finite difference equations with periodic boundary conditions.

$$
\begin{aligned}
& \underline{E}_{i}=\left(\underline{\underline{B}}_{i}-\underline{\underline{C}}_{i} \cdot \underline{\underline{E}}_{i-1}\right)^{-1} \cdot \underline{\underline{A}}_{i} \\
& \underline{F}_{i}=\left(\underline{B}_{i}-\underline{C}_{i} \cdot \underline{E}_{i-1}\right)^{-1} \cdot\left(\underline{D}_{-1}+\underline{C}_{i} \cdot \underline{F}_{1-1}\right) \\
& \underline{S}_{i}=\left(\underline{\underline{B}}_{i}-\stackrel{C}{I}_{i} \cdot \underline{\underline{E}}_{i-1}\right)^{-1} \cdot\left(\underline{\underline{C}}_{i} \cdot \underline{\underline{S}}_{i-1}\right)
\end{aligned}
$$

for $i=2,3, \ldots I$, Unce again, we use he boundary conditions to $f$ ix the values of $\underline{\underline{E}}_{1}, \underline{F}_{1}$ and $\underline{S}_{1}$. From Eq. (III.6.7) ano substitutirs $i=1$ into 
Eq. (III.6.8), we get

$$
\begin{aligned}
& \underline{E}_{1}=\underline{\underline{0}}, \\
& \underline{\mathrm{F}}_{1}=\underline{0}, \\
& \underline{\mathrm{S}}_{1}=\underline{\underline{I}},
\end{aligned}
$$

where $\underline{\underline{I}}$ is the identity matrix. Next, we need to fix the value of $\underline{u}_{\mathbf{i}}$. Before we can do this, we must introduce the following relation

$$
\underline{u}_{\mathbf{i}}=\underline{\mathrm{T}}_{\mathbf{i}} \cdot \underline{\mathrm{u}}_{1}+\underline{\mathrm{V}}_{\mathrm{i}}, 1=\mathrm{I}, \ldots, 3,2
$$

This is substituted into $\mathrm{Eq}_{\mathrm{q}}$. (III.6.8) for $\underline{u}_{i+1}$ yielding the relations

$$
\underline{\underline{T}}_{i}=\underline{E}_{i} \cdot \underline{\underline{T}}_{i+1}+\underline{S}_{i}
$$

and

$$
\underline{v}_{i}=\underline{E}_{i} \cdot \underline{v}_{i+1}+\underline{F}_{i}, i=I, \ldots, 3,2
$$

Next, the values of $\underline{\mathrm{T}}_{\mathrm{I}+1}$ and $\underline{\mathrm{V}}_{\mathrm{I}+1}$ are fixed by evaluating Eq. (III.6.11) at $\mathbf{i}=I+1$ and using $\mathrm{Eq}$. (III.6.7) yielding

$$
\underline{\mathrm{T}}_{\mathrm{I}+1}=\underline{\underline{\mathrm{I}}}
$$

and

$$
\underline{\mathrm{V}}_{\mathrm{I}+\mathrm{J} .}=\underline{\mathrm{O}} .
$$


Fina11y, we substitute $:=1$ into Eq. (III.6.1) and use Eq. (III.6.11) to eliminate $\underline{u}_{2}$ and the periodicity to eliminate $\underline{u}_{I}$. The value of $\underline{u}_{1}$ thus fixes as

$$
\begin{array}{r}
\underline{u}_{1}=\left(\stackrel{B}{=}_{1}-\stackrel{A}{=}_{1} \cdot \underline{T}_{2}-\underline{C}_{1} \cdot \underline{T}_{I}\right)^{-1} \\
\cdot\left(\underline{D}_{1}+\underline{A}_{1} \cdot \underline{v}_{2}+\underline{C}_{1} \cdot \underline{V}_{1}\right)
\end{array}
$$

Thus, our algorithm for periodic boundaries consists of three sweeps through the mesh. Pass one sweeps from $i=2,3 \ldots$, I solving Eq. (III.6.9) for E, $F$ and $\stackrel{\$}{=}$, using Eq. (III.6.10) as starting values. Pass two sweeps from $i .=1, I-1, \ldots 3,2$ solving En. (III.6.12) for $\underline{\underline{T}}$ and $\underline{\mathrm{V}}$ using Eq. (III.6.13) as starting points. Finaliy, we use Eq. (III.6.14) to Eix $\underline{u}_{1}$ and then solve Eq. (III.6.11) $\mathrm{f} \sim{ }_{L} \underline{u}_{i}$ across the mesh. Once again, the most time consuming part $=i$ the operation is the inversion of the ( $\underline{\underline{B}}-\underline{\underline{C}} \cdot \underline{\underline{E}})$ matrix at every grid point.

\section{III.7 Boundary Conditions}

This section will describe the types of boundary conditions implemented in IMP. As was mentioned in Section III.6, the block tridiagonal solver requires a boundary condition of the form

$$
\underline{G} \cdot \underline{\mathrm{u}}_{\mathrm{B} . P .}=\stackrel{H}{\underline{H} \cdot \underline{\underline{u}}_{I N T}} \mathrm{~N} \underline{\mathrm{J}} .
$$

where $\underline{u}_{\mathrm{B} . P .}$ is the bounriary point and $\underline{u}_{\mathrm{INT}}$ is the next interior point. A11 forms of Neumann, Dirichlet and mixed boundary conditions can be represented 
by the equation

$$
\underline{\underline{\alpha}} \cdot \frac{\partial \underline{u}}{\partial n}+\underline{\beta} \cdot \underline{\underline{u}}=\underline{\gamma},
$$

where $\frac{\partial}{\partial n}$ is the derivative normal to the boundary. If we finite difference Eq. (III.7.2) via the rules of Section III.4, we obtain

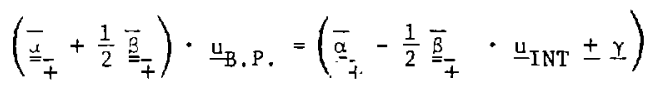

where the top sign is taken at the $i=1$ boundary and the bottom sign is taken at the $i=I$ boundary. We see then that Eq. (III.7.1) allows a verv general class of boundary conditions to be implemented.

\section{III.7.1 GHOST Point Boundary Conditions}

One consideration is the accuracy of the boundary conditions. If the boundary point is picked so that the wall lies on the last mesh point, the differences given in Eq. (III.7.3) are one-sided. This leads to first order accurate boundary values. If we choose the last mesh point so that the wall Iies halfway between it and the next interior point, we get second order accurate difference approximations to the derivatives. IMP uses the second method of placing "ghost" points outside the rall as one form of its $t$ indary conditions.

To assign values for $\underline{G}, \underline{H}$ and $I$, we will assume that the plasma is surrounded by a perfectly conducting, adiabatic wall. The electro- 
magnetic field equations give us, for a perfectly conducting wall,

$$
\hat{n} \cdot \underline{B}=0
$$

and

$$
\hat{n} \times \underline{E}=0 \text {, }
$$

where $\hat{n}$ is the unit normal vector outward from the wall. That is, the zormal magnetic field and tangential electric fields vanish. For the rest of this section, we assume that the wall exists at $x_{1}=x_{w}$ were $\mathrm{x}_{1}$ is some arbitrary orthogonal curvilinear coordinate. The results, of course, are true for any coordinate we choose. Equation (III.7.1.1) gives us, then, that

$$
B_{1_{W}}=0
$$

Next, we write Dhm's law and cross it with $\hat{n}$ to give us

$$
\hat{n} \times \underline{E}_{w}=\hat{n} \times\left(\underline{v} \times \underline{B}-\frac{\eta}{S} \nabla \times \underline{B}\right)_{w}=0 .
$$

Look:ng at the tangential component. of this yields (remembering that $\hat{n}$ is in the $x_{1}$ direction)

$$
\mathrm{E}_{2}=\left(\mathrm{v}_{3} \mathrm{~B}_{1}-\mathrm{v}_{1} \mathrm{~B}_{3}-\frac{\Pi}{\mathrm{S}}\left(\nabla \times \underline{B}_{2}\right)_{\mathrm{w}}=0\right.
$$


and

$$
E_{3}=\left(v_{1} B_{2}-v_{2} B_{1}-\frac{\Pi}{S}\left(\nabla \times \underline{B}_{3}\right)_{w}=0 .\right.
$$

We may use Eq. (III.7.1.3) to eliminate the ${ }^{B}{ }_{1_{w}}$ terms from (III.7.1.5) leaving

$$
\left(\mathrm{v}_{1} \mathrm{~B}_{3}+\frac{n}{\mathrm{n}}\left(\nabla \times \underline{B}_{2}\right)_{w}=0\right.
$$

and

$$
\left(v_{1} B_{2}-\frac{\eta}{S}\left(\nabla \times \underline{B}_{3}\right)_{w}=0 .\right.
$$

Since w't 1. ve a solid wall, we may write

$$
\hat{n} \cdot \underline{v}_{w}=0,
$$

giving us

$$
v_{1_{w}}=0
$$

which furthe: simplifies (III.7.1.6) to

$$
\left(\nabla \times \underline{B}_{2}=\left(\nabla \times \underline{B}_{3}=0 .\right.\right.
$$


Expanding (III.7.1.9) and remembering that $B_{1_{3}}=0$ for all values of $x_{2}$ and $x_{3}$ yields

$$
\frac{i h_{2} B_{2}}{i x_{1}}=\frac{3 h_{3} B_{3}}{\exists x_{1}}=0
$$

or, more generally,

$$
\frac{h_{T} T^{B} T}{\partial n}=0
$$

where the $\mathrm{T}$ subscript refers to the component of the field tangential to the wall.

Next, we evaluate the normal component of the momentum equation at the wall, obtaining

$$
\begin{aligned}
\frac{\partial \rho v_{1}}{\partial t} & +\frac{1}{h_{1} h_{2} h_{3}}\left\{\frac{\partial}{\partial x_{1}}\left(h_{2} h_{3} \rho v_{1}^{2}\right)+\frac{\partial}{\partial x_{2}}\left(h_{1} h_{3} \rho v_{1} v_{2}\right)\right. \\
& +\frac{\partial}{\partial x_{3}}\left(h_{1} h_{2} \rho v_{1} v_{3}\right)+h_{3} \rho v_{1} v_{2} \frac{\partial h_{1}}{\partial x_{2}}+h_{2} \rho v_{1} v_{3} \frac{\partial h_{1}}{\partial x_{3}} \\
& \left.-\left[h_{3} \rho v_{2}^{2} \frac{\partial h_{2}}{\partial x_{1}}+h_{2} \rho v_{3}^{2} \frac{\partial h_{3}}{\partial x_{1}}\right]\right\}+\frac{1}{2 h_{1}} \frac{\partial p}{\partial x_{1}} \\
& +\frac{B_{2}}{h_{1} h_{2}}\left\{\frac{\partial}{\partial x_{1}}\left(h_{2} B_{2}\right)-\frac{\partial}{\partial x_{2}}\left(h_{1} B_{1}\right)\right\} \\
& +\frac{B_{3}}{h_{1} h_{3}}\left\{\frac{\partial}{\partial x_{3}}\left(h_{1} B_{1}\right)-\frac{\partial}{\partial z_{1}}\left(h_{3} B_{3}\right)\right\}=0
\end{aligned}
$$


Using Eqs. (III.7.1.3), (III.7.1.7) and (III.7.1.11) reduces (III.7.1.12) to

$$
\begin{aligned}
& -\frac{1}{h_{1} i_{2} h_{3}}\left\{h_{3}: v_{2}^{2} \frac{3 h_{2}}{\partial x_{1}}+h_{2} \rho v_{3}^{2} \frac{3 h_{3}}{i x_{1}}\right\}_{w} \\
& +\frac{1}{2 h_{1}}\left(\frac{\partial P}{3 x_{1}}\right)=0,
\end{aligned}
$$

which is a boundar: condition on pressure. We take the well known "free slip" boundary condicion (see Roache ${ }^{68}$ ) to fix the vel xity tangenial to the wall,

$$
\frac{h T^{v} T}{i_{n}}=0
$$

Finally, we use the adiabaticitv of the wall which allows roc thelt flow through the wall,

$$
\frac{3 \mathrm{~T}}{\partial_{\mathrm{n}}}=0
$$

coupled with our normal thermodynamic relationship

$$
P=\rho T,
$$

to give

$$
\frac{\partial\left(\frac{P}{\rho}\right)}{\partial n}=0 .
$$


We now have a full set of nonlinear boundary conditions for u. in flrimar then the boundary conditions to be applied at a perfectly (1)ulketing, adiathatic, invisid wall are:

$$
\begin{aligned}
& n_{n}=r \quad: \\
& \frac{\left.y^{1}\right]}{21}=, \quad: \\
& n-1 ; \quad \text {; } \\
& \because " !+1= \\
& \left({ }^{n}\right)=i \quad \text { : }
\end{aligned}
$$

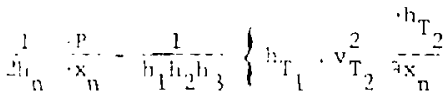

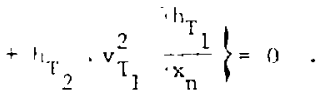

B: applying the averaging and differencing operators oi section $1 \mathrm{H}, 3$ 11 Eqs. (III.7.1.18) wo have a set of equations whints represent nur buntary condition. We then use these equations and form our cell interface Jacolians which yields, at the $\mathrm{i}=1$ houndar", 
${ }_{=}^{G}=\left(\begin{array}{cccccccc}1 & 0 & 0 & 0 & 0 & 0 & 0 & 0 \\ 0 & \mathrm{~h}_{2} & 0 & 0 & 0 & c & 0 & 0 \\ 0 & 0 & \mathrm{~h}_{3} & 0 & 0 & 0 & 0 & 0 \\ 0 & 0 & 0 & 1 & 0 & 0 & 0 & 0 \\ 0 & 0 & 0 & 0 & \mathrm{~h}_{2} & 0 & 0 & 0 \\ 0 & 0 & 0 & 0 & 0 & \mathrm{~h}_{3} & 0 & 0 \\ 0 & 0 & 0 & 0 & 0 & 0 & -\mathrm{P}_{2} & 0_{2} \\ 0 & 0 & 0 & 0 & \mathrm{~A}_{\mathrm{L}} & \mathrm{B}_{\mathrm{L}} & \mathrm{C}_{\mathrm{L}} & 1\end{array}\right)$,

(III.7.1.19)

where

$$
\begin{aligned}
& A_{L}=\frac{2 \hat{A}_{\bar{n}} \overline{\mathrm{v}}_{2}}{\overline{\mathrm{h}}_{2}} \frac{\overline{\mathrm{h}} \mathrm{h}_{2}}{\overline{\mathrm{i} \mathrm{x}_{1}}} \text {, } \\
& \mathrm{B}_{\mathrm{L}}=\frac{2 \mathrm{i} \bar{v}_{3}}{\mathrm{~h}_{3}} \frac{\partial \mathrm{h}_{3}}{\partial \mathrm{x}_{2}} \\
& c_{L}=\frac{2 \hat{i}_{-}}{{\overrightarrow{h_{2}}}_{2} \bar{h}_{3}}\left\{\bar{h}_{3} \bar{v}_{2}^{2} \frac{\partial h_{2}}{\partial x_{1}}+\vec{h}_{2}, \bar{v}_{3}^{2} \frac{\partial h_{3}}{\partial x_{1}}\right\} ;
\end{aligned}
$$

and

$$
\stackrel{H}{=}=\left(\begin{array}{cccccccc}
-1 & 0 & 0 & 0 & 0 & 0 & 0 & 0 \\
0 & n_{2} & 0 & 0 & 0 & 0 & 0 & 0 \\
0 & 0 & h_{3} & 0 & 0 & 0 & 0 & 0 \\
0 & 0 & 0 & -1 & 0 & 0 & 0 & 0 \\
0 & 0 & 0 & 0 & h_{2}{ }_{2} & 0 & 0 & 0 \\
0 & 0 & 0 & 0 & 0 & h_{3} & 0 & 0 \\
0 & 0 & 0 & 0 & 0 & 0 & 0 & 0 \\
0 & 0 & 0 & 0 & D_{L} & E_{L} & F_{i} & 1
\end{array}\right)
$$


where

$$
\begin{aligned}
& \mathrm{D}_{\mathrm{L}}=\frac{-2 \Delta \overline{\mathrm{\rho}} \overline{\mathrm{v}}_{2}}{\overline{\mathrm{h}}_{2}} \frac{\partial \mathrm{h}_{2}}{\partial \mathrm{x}_{1}}, \\
& \mathrm{E}_{\mathrm{L}}=\frac{-2 \Delta \overline{\mathrm{n}} \overline{\mathrm{v}}_{3}}{\overline{\mathrm{h}}_{3}} \frac{\partial \mathrm{h}_{3}}{\partial \mathrm{x}_{1}}, \\
& \mathrm{~F}_{L}=\frac{-2 \Delta}{\overline{\mathrm{h}}_{2} \overline{\mathrm{h}}_{3}}\left\{\overline{\mathrm{h}}_{3} \overline{\mathrm{v}}_{2} \frac{\partial \mathrm{h}_{2}}{\partial \mathrm{x}_{1}}+\overline{\mathrm{h}}_{2} \overline{\mathrm{v}}_{3} \frac{\partial \mathrm{h}_{3}}{\partial \mathrm{x}_{1}}\right\},
\end{aligned}
$$

and

$$
\underline{\mathrm{J}}_{\mathrm{L}}=0 \quad \text {. }
$$

Simlarly, for the $\mathbf{i}=\mathbf{I}$ boundary, we have

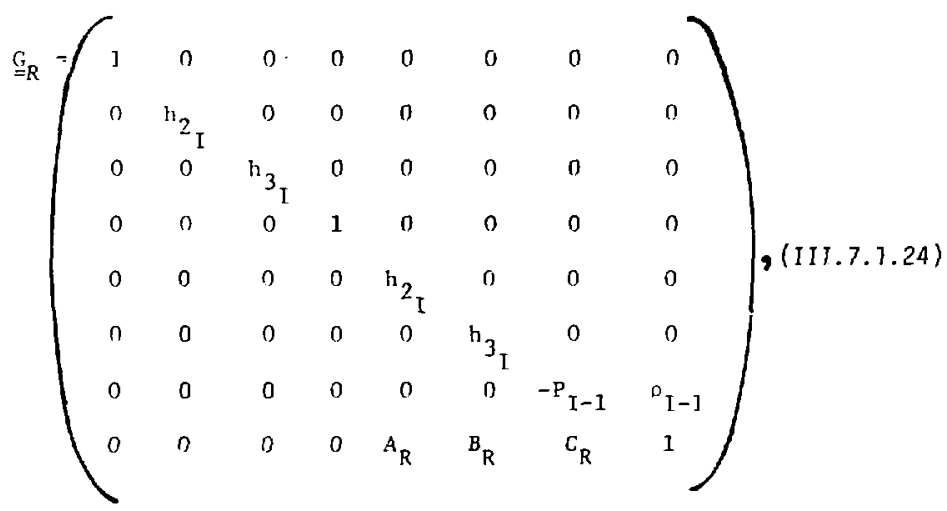


where

$$
\begin{aligned}
& A_{R}=\frac{-2 \Delta \bar{\rho} \bar{v}_{2}}{\bar{h}_{2}} \frac{\partial h_{2}}{\partial x_{1}}, \\
& B_{R}=\frac{-2 \Delta \bar{\rho} \bar{v}_{3}}{\bar{h}_{3}} \frac{\partial h_{2}}{\partial x_{1}}, \\
& C_{L}=\frac{-2 \Delta}{\bar{h}_{2} \bar{h}_{3}}\left(\bar{h}_{2} \bar{v}_{3}^{2} \frac{\partial h_{3}}{\partial x_{1}}+\bar{h}_{3} \overline{v_{2}} \frac{\partial h_{2}}{\partial \mathrm{v}_{1}}\right) ;
\end{aligned}
$$

and

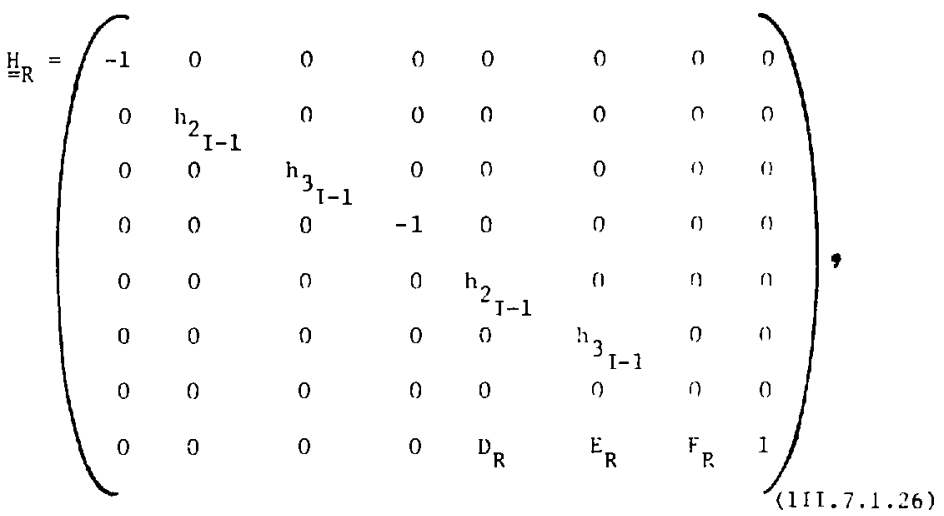

where

$$
\begin{aligned}
& \mathrm{D}_{\mathrm{R}}=\frac{2 \Delta \bar{\rho} \overline{\mathrm{v}}_{2}}{\overline{\mathrm{h}}_{2}} \frac{\partial \mathrm{h}_{2}}{\partial \mathrm{x}_{1}}, \\
& \mathrm{E}_{\mathrm{R}}=\frac{2 \Delta \bar{\rho} \overline{\mathrm{v}}_{3}}{\overline{\mathrm{h}}_{3}} \frac{\partial \mathrm{l}_{2}}{\partial \mathrm{x}_{1}},
\end{aligned}
$$




$$
F_{R}=\frac{2 \Delta}{\bar{h}_{2} \bar{h}_{3}}\left(\bar{h}_{2} \bar{v}_{3}^{2} \frac{\partial h_{3}}{\partial x_{1}}+\bar{h}_{3} \bar{v}_{2}^{2} \frac{\partial h_{2}}{\partial x_{1}}\right) \text {, }
$$

and

$$
\mathrm{J}_{\mathrm{R}}=\mathrm{C} \text {. }
$$

\section{III.7.2 Flux Surface Boundary Conditions}

Another approach for setting the boundary conditions is to consider the physical interpretation of the boundary. Since we are modeling a perfectly conducting, adiabatic, invisid wall which lies on a flux surface of the plasma, we can make the following statements: ${ }^{54}$

1. The wall allows no fluxes into or out of the computational region.

2. The wall exerts a force that exactly balances any residual $P$ $+\mathrm{B}^{2}$ force.

We take a mesh which has its 1-sit point half a cell away from the wall (See Fig. III.4). The values of the state vector, $\underline{u}$, are not needed at the wall, only the fluxes and forces are required chere. The differencing techniques outlined in Section III.3 were chosen to take advantage of this property. The conditions of statements 1 and 2 above are implemented merely by setting the symmetric and antisymmetric matrices $\stackrel{A}{=}$ and $S$ of Section III.5 to zero at the wall-interface. We thus have a very easily implemented boundary condition that is physically motivated. It is this physically motivated boundary condi- 
tion that is most often used when ruming IMP and we have consistent1y seen good results when using it. A more general form of this physical boundary condition is used by Lindemutin in the ANIMAI ${ }^{69}$ code. In ANIMIL, the plasma $\mathrm{P}+\mathrm{B}^{2}$ force is also checked to detect plasma separating from the wall. When this occurs, a background plasma is jetted in to fill the vold. This wall-separation type of boundarv condition may eventually be incorporaced into the IMP code.

\section{III.8 Miscellineous Computation Problems}

\section{III.8.1 Advection Terms}

As was pointed out by Brackbill, 40 the nonlinear advection terms $\left(\frac{\partial}{\partial t}+\underline{v} \cdot \nabla\right)$ in the MHD equations cause a large number of problems. While the Crank-Nicholson and leapfrog approximation of the advection terms are stable according to linear theory, they are "explosively unstable when numerically solved". The source of the instahilicy is traced to the truncation error in the differcnce equations causing antidiffusion. In order to offset this antidiffusion, small, purely numerical, diffusion terms are added to the continuitv equation, the force equation and the energy equation. The form of clese terms is

$$
\frac{\partial u}{\partial t}+\nabla \cdot(F(u)-c \nabla u)=0
$$

where $c$ is a constant which is typically of order $1 \times 10^{-4}$. These terms are differenced as FLUX terms in Section III.3. Experience has shown that the inclusion of these artifical diffusion terms has resulted in 
the ability to run with much larger timestep sizes and with increased numerical stability.

\section{III.8.2 Iteration Controls}

The current version of IMP advances the mesh one row at a time. In this manner, we are able to allow rows with large changes to tterate many times while rows that have little or no change elther do not iterate at all or only iterate once or twice. We are able to do this because our choice of ADI techniques, along with the method of differencing the mixed derivative and product of derivative terms, decouples the rows of the mesh. The iterations are carried to convergence by testing for the maximum variation between iterations versus the arerage value of the iterations

$$
\underline{\varepsilon}=\operatorname{MAX}\left[5\left(\underline{u}_{i}-\ell-1{\underline{u_{i}}}_{1}\right) /\left(\underline{u}_{i}^{\ell}+\ell \underline{u}_{i}\right)\right]
$$

These are subjected to a floor value such that if ${ }^{\ell-1} u_{i}$ is less than the floor, then floor value is used for ${ }^{\ell} \underline{u}_{i}$ in the denominator of III.8.2.1. Finally, the maximum value of $E$ is compared with the allowed convergence criterion and, if $\varepsilon$ is less than the criterion, the row is declared converged. Typical values for the convergence criterion is $\varepsilon<1 \times 10^{-5}$.

\section{III.8.3 Timestep Controls}

The main purpose of using implicit techniques was to allow the use of large timesteps. Early versions of IMP used a static timestep. 
They experienced the difficulty that the problem's timestep was limited by the timesteps needed during the extreme nonlinear phases of the code's run. In order to bypass this constraint, a dynamic timestepping algorithm was implemented. This algorithm allowed the input of an initial timestep and maximum and minimum allowable timesteps. The code has a number of ways to choose its timcstep $(\Delta t)$.

The first method monitors the maximum number of iterations required $(\mathrm{N})$ to converge the rows of the mesh. This number is compared with an input allowed number of iterations (M). If the number needed is less than the input number $(N<M)$, then the timestep is increased by some factur (usually $10 \%$ ). If the $\Delta t$ is increased for a number of consecutive timesteps, the increment factor is doubled. This doubling continues until the "ideal" timestep has been found. If $N: M$, then the timestep is decreased. If $N>M+1$, then a recycle is performed. That is, the new timestep is rejected and we redo the timestep with a smaller $\Delta t$, If $N=M$, we also increment it by a smail amount to try and find the largest possible $\Delta t$.

The second method is to monitor the maximum change of the variables on the mesh (subject to some floor condition below which we do not check the change). In this method, we have a numiser of subcontrols. We want the maximum variation on the mesh, which is calculated as

$$
\underline{v}_{\max }=\sum_{i, j, k} \max \left(\underline{u}_{i, j, k}^{n+1}-\underline{u}_{i, j, k}^{n} / \underline{u}_{i, j, k}^{n}\right),
$$


to be as close as possible to some allowed change $c$. If $V_{\text {max }}$ is less than $.85 c$ we increase the timestep. If $v_{\max }$ is greater than $1.5 \mathrm{c}$ we recycle. Otherwise if $c<v_{\max }<1.5 \mathrm{c}$, we decrease the timestep by the formula

$$
\Delta t=.8 \Delta t\left(\mathrm{c} / V_{\max }\right) .
$$

The subcontrols in this method allow one to choose to check the variation of momentum rather than the change of velocity. They also allow for time variations in the velocity/momentum floor values. The reason for allowing this variation is that we are interested in following the evolution of instabilities. In instabilities, the velocity is always increasing and, where we initlally had the velocity floor set to $5 \%$ of the maximum on the mesh, we can end up being limited by the change in the slowest velocity on the mesh. By constantly revising the floor value we are able to hold it to $5 \%$ of the maximum velocity. This has resulted in increases of $70-150 \%$ in our running values of $\Delta t$.

The aforementioned timestep controls are only first approximations at a general timestep control algorithm. Other factors that can be Lested are the energy conservation, changes in interface fluxes, or truncation error analysis. Which of these are necessary and, in the case of the truncation error analysis, the method of implementation are not currently understood. Future work in this area may result in even more dramatic increases in timestep size. 


\section{CHAPTER IV \\ THE INTERNAL KINK IN CARTESIAN GEOMETRY}

\section{1 Introduction}

The first three-dimensional application of our midel is the simulation of the $m=1, n=1$ internal kink instability in Cartesian coordinates. Figure IV.1 shows the computational region that is used in this chapter.

As has been discussed in Chapter I, the internal kink instability is the most dangerous to plasma confinement. The mode is unstable whenever the $q=1$ surface 1 ies inside the $\mathrm{plasma}$. Figure IV. 2 shows typlcal radial profiles of the current $j_{z}(r)$, safety factor $q(r)$, and the lowest order Iadial eigenfunction of the displacement $\xi(r) .{ }^{62}$ The displacement for the mode is entirely cortained within the singular surface at $r=r_{S}$. The linear growth rate of this mode is given analytically to be of order $\mathrm{k}_{z}^{3} \mathrm{r}^{2} \mathrm{v}_{\mathrm{A}}$. In the simulations to be presented, we have chosen $k_{z}=n / k=1$ and $r=r_{S} \approx \cdot 8$. Thus, we can expect growth rates to be of order $.5 v_{A}$. This shows that the kink mode is growing on the Alfven timescale ir: the problem.

The internal kink mode has been the subject of a great deal of computational study, both in the linear and nonlinear regimes. These studits have been performed by Bateman et al. ${ }^{9}$ and Hicks ${ }^{55}$ in the limit of ideal MHD. The results of our model is compared with these studies as an independent check.

The first case run is the use of a small velocity field to perturb an analyu ic equilibrium. The resistivity in our model is set to zero. The 
perturbation is allowed to evolve until pure exponential growth is observed. This rate of growth and the form of the eigenmode is compared with the linear code of Bateman, which has been shown to give good agreement with theorv. Our model produces growth rates that are within $2 \%$ of the Jinear results. The perturbation is then allowed to continue its growth until nonlinear saturation of the mode occurs. The amplitudes of the velocity at saturation are then compared with those obtained by Hicks and are found to bo in good agreement.

Next, an eigenmode obtained from the linear code is used as the perturhation in IMP. The object is to determine if the eigenmodes of the lincar code produce an immediate exponential growth in the nonlinear model. Tht linear eigenmodes, started at relatively large magnitudes, are to be u"ed ats the initial perturbations for IMP. This allows the bypassing of most of the initial linear growth regime in future saturation studies. The eigenmodes from the linear code do display the desired immediate exponential growth.

After performing the ideal MHD comparisons, we intioduce a finite (Spitzer ${ }^{65}$ ) resistivity. This causes both resistive diffusion of the magnetic field and Ohmic heating of the plasma. Both of these effects cause spontaneous flows in the plasma. In order to allow the maintenance of a constant current in the plasma, a background electric field can be imposed, This external Field exactly balances the resiscive diffusion of the equilibrium magnetic fields. Once determined, the external field remains constant for the remainder of the simulation. In a like fashion, a nonphysical cooling term can be added to the energy equation to balance 
the ohmic heating. While the imposition of the extemal electric field can be Justifled in some cases, the nonphysical cooling cannot be justified and is added only to allow the usr of the velocity flow field and the kinetic energy as diagnostics. A simulation is also performed without the cooling term and also one without the external electric field. In these cases, the eigenmode grows to dominate the spontaneous flows and the growth rate of the instability is only slightly modifled by the Inclusion of the nonphysical terms. The resistivity is found to enhance the growtt of mode while the final saturation veloctties are slightly depressed due to energy golng into heating the plasma.

\section{2 Equilibrium}

The equilibrium configuration is determined by the solution of the momentum equatior. (II.2.12.b) under the condition of a stationary sieady state,

$$
\frac{1}{2} \nabla P=(\nabla \times \underline{B}) \times \underline{B} .
$$

The values of the magnetic field and pressure that satisfy this are given by $^{21}$

$$
\begin{aligned}
& B_{x}=-\frac{\pi}{2} \psi_{c} \cos \left(\frac{\pi}{2} x\right) \sin \left(\frac{\pi}{2} y\right) ; \\
& B_{y}=\frac{\pi}{2} \psi_{c} \sin \left(\frac{\pi}{2} x\right) \cos \left(\frac{\pi}{2} y\right) ; \\
& B_{z}=1
\end{aligned}
$$




$$
P=\frac{1}{2}\left(\frac{\pi}{2}\right)^{2} \psi_{c}^{2} \cos ^{2}\left(\frac{\pi}{2} x\right) \cos ^{2}\left(\frac{\pi}{2} y\right) .
$$

We also assime a constant initial density $p=1$. The safety factor per unit wavelength on the axts can be expressed as

$$
q_{c}=\frac{2 B z_{c}}{\pi t J_{c}}
$$

where $\mathrm{J}_{z_{c}}$ is the current on axis and $t \equiv \lambda / 2 \pi$ is the wavelength of the Instaility. The central current is obtained by evaluating $\nabla \times B$ at $x=y=0$ yleiding

$$
J_{z_{c}}=2\left(\frac{\pi}{2}\right)^{2} \psi_{c} .
$$

We are able specify our equilibrium by choosing $\pi$ and $q_{c}$. We considcr here a short pipe such that $t=1$ and $q_{c}=.6$.

\section{3 Initial Conditions}

The infial equilibrlum can be perturbed by two methods. The first method is to generate two counter-ro'iting vortices in $x-y$ plane. The sense of the vortices are then twisted as we proceed along in the $z$ direction. This is accomplished by first specifying the velocities on the $z=0$ and $z=\cdot r / 4$ planes. These velocitles are

$$
v_{x=0}(x, y)=v_{y}(x, y)=\frac{x y}{|x y|} \sin ^{2} \pi x \sin ^{2} \pi y
$$




$$
\begin{aligned}
& -99 \text {. }
\end{aligned}
$$

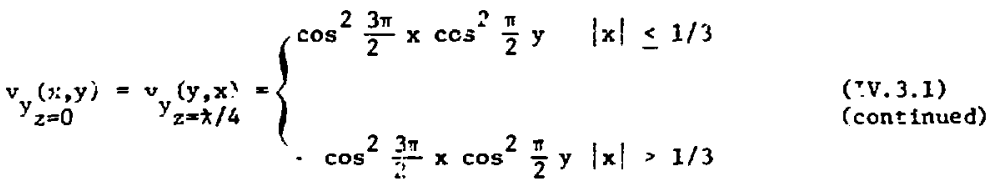

The velocities are then conblned by

$$
\begin{aligned}
& v_{x}(x, y, z)=c\left(v_{x_{z=0}}(x, y) \cos 2 \pi z-v_{x_{z=7 / 4}}(x, y) \sin 2 \pi z\right) \\
& v_{y}(x, y, z)=c\left(v_{y}(x, y) \cos 2 \pi z-v_{y=0}(x, y) \sin z \pi z\right)
\end{aligned}
$$

to obtain the final per. :t ion velocities where $C$ is a constant which determines the inftlal amplitude. We have generally used $C \approx 10^{-4}$. The sense of these velocity vortices spiral at the same pitch as the magnetic fleld Ifnes, tnus assuring us that the pizturbation will al ign tiself with the r. agnetic ifeld.

The second method for perturbing the equilibrium fs to use the eigenmodes of $\underline{B}_{1}, \underline{v}_{1}$, and $P_{I}$, where the $I$ subscrfpt den ces a perturiation quartity, gererated by the linear code. These elgenmodes are generated on the $z=0$ ard $z=\pi / 4$ planes. We therefore use a corbination technique that is similar 'n iorm to (IV.3.2):

$$
\phi(x, y, z)=C^{\prime}\left(\phi_{0}(x, y) \cos 2 \pi z \cdots \phi_{1}(x, y) \sin 2 \pi z\right),
$$

where $\phi 1 s$ any of the perturbation quantities, $\phi_{0}+1$. 1 ss valua on the $z=0$ plane and $\phi_{1}$ is 1 cs value on the $z=\pi / 4$ plane, and $C^{\prime}$ is a consta th that that sets the magnituce of the perturbation :o be used. 


\section{4 Boundary Conditions and Normalization}

Since the walls are a flux surface we may use the adiabatic, perfectly conducting wall boundary conditions glven in Chapter III. We have checked the consistency of tinese boundaries by rerunning the same problems with the "ghost" points of Chapter III to see if there are any discrepancles. The ghost point conditions used are the ones given by

$$
\begin{aligned}
& \left.\frac{\partial B_{T}}{\partial n}\right|_{W}=\left.\frac{\partial v_{T}}{\partial n}\right|_{W}=0 \\
& \left.B_{n}\right|_{W}=\left.v_{n}\right|_{W}=0 \\
& \frac{\partial T}{\partial n}=\frac{\partial P}{\partial n}=0
\end{aligned}
$$

The scale factors for Gartesian coordinates are all constant. The internal coordinate system used is normalized to be

$$
\begin{aligned}
& -1 \leq x_{1}, x_{2} \leq+1 \\
& 0 \leq x_{3} \leq+1
\end{aligned}
$$

where $x_{1}$ corresponds to the $x$ direction, $x_{2}$ to the $y$ direction and $x_{3}$ to the $z$ direction. The normalization is chosen such that the half width in the $x$ direction is unfty. This is accomplished by setting

$$
\begin{aligned}
& h_{1}=1 \\
& h_{2}=b / a, \\
& h_{3}=2 \pi \lambda
\end{aligned},
$$


where a is the half width in the :i direction, $b$ is the half width fa ihe $y$ direction and $\lambda$ is the wavelength in units of a (see Fig. IV.1).

\section{IV.5 Dlagnostics}

The principle diagnostic tools used In IMP are time histories of the kinetic energy of the system and the Fourfer components of the pressure along the $z$ direction. The growth rate $Y$ is calculated from the se time historles. At speciflc points in time, vector plots of the velority and magnetlc fields on $x-y$ planes are generated, as well as contour plots of the pressure, density, temperature, and current density $f_{z}$ on $x-y$ planes. Efficiency of the computation is monitored by time history plots of the timestep size and Courant number (where $C=1$ is the maxinum step size for an explicit code).

As was stated above, the growth rate is calculated from the time historles of the kinetic energy of the system and from the Fourler components of the pressure. The growth rate of a quantity u at a point in time, based on a function $f\left(=f\left(u^{2}\right)\right)$, is given by

$$
\gamma=\frac{1}{2} \frac{d \ln f}{d t}=\frac{1}{2} \frac{1}{f} \frac{d f}{d t}
$$

The factor of $1 / 2$ is to take into account the fact that the function $f$ depends on a square of a plasma state function (e.g., $f=k f n e t i c$ energy $\left.\propto \rho v^{2}\right)$. 
The Fourfer analysis of the pressure in the periodic $\left(x_{3}\right)$ direction is

$$
\begin{aligned}
& P\left(x_{1}, x_{2}, x_{3}\right)=p_{0}\left(x_{1}, x_{2}\right)+\sum_{n=1}^{N}\left(p_{n}\left(x_{1}, x_{2}\right) \cos 2 \pi n x_{3}\right. \\
& \left.+q_{n}\left(x_{1}, x_{2}\right) \sin 2 \pi n x_{3}\right),
\end{aligned}
$$

where

$$
\begin{aligned}
& p_{0}\left(x_{1}, x_{2}\right)=\int_{0}^{1} P\left(x_{1}, x_{2}, x_{3}\right) h_{2} d x_{3} \\
& p_{n}\left(x_{1}, x_{2}\right)=\int_{0}^{1} P\left(x_{1}, x_{2}, x_{3}\right) \cos \left(2 \pi n x_{3}\right) h_{x} d x_{3} \\
& q_{n}\left(x_{1}, x_{3}\right)=\int_{0}^{1} P\left(x_{1}, x_{2}, x_{3}\right) \sin \left(2 \pi n x_{3}\right) h_{3} d x_{3}
\end{aligned}
$$

The power in a given mode is

$$
E_{n}=\alpha \iint\left(p_{n}^{2}+q_{n}^{2}\right) h_{1} h_{2} d x_{1} d x_{2},
$$

where $\propto$ is a constant of proportionality. Thus, the gowth rates in specific $n$ modes are given by

$$
\gamma_{F_{n}}=\frac{1}{2 E_{n}} \frac{d E_{n}}{d t}
$$

The two growth rate calculations are used for comparison with the linear code results. The growth rate based on the kinetic energy $\gamma_{\mathrm{KE}}$ and 
the veloctiy field plots are not able to follow the early time growth of Instabilities in the cases of a) finfte resistivity and b) lack of absoiute force balance in the equilibrium. Case (a) has been discussed. Case (b) can arise even when we start from an analytic equilibrium. This is due to the inherent error $\left(0\left(\Delta x^{2}\right)\right.$ ) in the finite difference representation. A set of force correction terms, based on equilibrium torce balance only, have been added to the momentum equation to alleviate this problem. Simulations with identfcal inftlal conditfons, oniy varying by the inclusion of these terms, have been performed. The incluston $c$. the force balance terms did not affect either the linear growth rate or the form of ihe velocity fields as compared to those found once the Instability had grown to dominate the background flow in the case without force balance terms.

The Fourier mode plots and growth rates, on the other hand, are able to follow the growth of the instabllity even in the early times. This is because the lack of force balance and the effect of re: istivity are axisymmetric and, thus, their effects are seen only in the $n=0$ mode.

\section{6 Results}

IV.6.1 Veloctty Field Perturbation, Ideal MHD

In tilis case, $C$ in Eq. IV.3.2 is set to $10^{-4}$. The equilibrium is chosen to have the $q$ per unit wavelength on axis to be .6 , and the wavelength $t=1$. The growth rate calculated for this equilibrium by the linear code is $Y_{\text {lin }}=.49$. Figure IV.3 shows the initial velocity field perturbation on the $z=0$ plane (IV.3.a) and the $z=\lambda / 4$ plane (TV. 3.b). In Fig. IV.4, the total energy (trace labeled E), internal 
energy (trace I), the mannetic field energy (trace B), and the kinetic ezergy (trace $k$ ) are plotted versus time on a semilog graph. In this simulation, the timestep is kept at a small constant value. wh: ascillations in the kinetic energy at early times can be at: buted to magnetoacoustic waves transiting the system. As time progresses, the $m=1, n=1$ mode establishes itself and the energy being depositied in the mode by the magnetic field dominates the energy fluctuations cathed by the waves. During this period of time $(t=7$ to $t=19)$ the kinetic energy time becomes linear. The growth rate calculated from the kiretic energy is given in Fig. IV.5, which converges at $r=14$, in a value of ${ }_{r_{K}}=.48$, which is within $2 \%$ of the linear code's result. Figure IV.6 shows the velocity fields on the $z=0$ and $z=i / 4$ planes duriug the pure exponential growch period, while Fig. IV.7 shows the vilues of the state variables across a lue defined bv $-1: x \leq 1, y=$ $z=0$. The mode then ceases exponentiai growth at $t=18$, where the kinetic energy is $1 \%$ of the total energ: in the problem. The velocity fields at this time are given in Fig. IV. 8 while the fi.lal value attained after 23 transits is shown in Fig. IV.9. The poloidal veloity attained by Hicks was of order .5 which is in good agreement with the final velocity attained in our model.

\section{IV.6.2 Perturbation by Linear Eigenmodes, Ideal MHD}

The eigenmodes obtained from the linear code are shown in Fig. IV.10. The spatial dependence of these modes agrees quite well with those obtained in the previous section. The constant $C^{\prime}$ in Ey. (IV.3.3) is set to $10^{-4}$ in order to assure the perturbation is in the 
linear regime. The mode grows exponentially immediately, as can be seen in Fig. IV.11, which gives the kinetic and Fourier mode energies. The growth rates based on these energies are shown in Fig. IV.12. They initially display variations from the linear growth rates but after a few Alfven transits the growth rate, based on both diagnostics, converges to $\gamma=.53$. This is within $2 \%$ of the value cbtained by the linear code of .54. The initial jitter in the growth rates in our model can be traced to the differences in the linear versus nonlinear equations and to different finite difference techniques used in the two codes.

\section{IV.6.3 R-sistive Plasma Long Time Simulation}

The first case run with finite resistivity used only the velocity field perturbation $\left(v_{\max }=10^{-4}\right.$ ) with $S=1000$ and the external electric field and nonphysical cooling terms enabled. Figure IV. I 3 shows the time history of the energy. Initially energy is taken crotit the perturbation and deposited in the plasma. By $t=2$ this trend is: reversed as the $m=1, n=1$ mode begins to grow. The exponential growth is well established by $t=5$. Fig. IV.14 shows the velocitv fields on the $z=0$ and $z=t / 4$ planes at $t=9.6$. This is miduay through the exponential growth regime. Growth continues until $t=20$ at which point nonlinear saturation occurs. At this point, the wall is stabilizing the mode. Peak pcloidal velocities of order . $4 \mathrm{v}_{\mathrm{A}}$ are attained. After saturation of the 1,1 mode, higher toroidal modes can be detected in the Fourier energy plots. Finally, the plasma relaxes to a state with an extremely slowly growing $\mathrm{m}=3, \mathrm{n}=1$ mode at $\mathrm{t}=$ 90. Figure IV.15 shows the velocity fields of this 3,1 mode on the $z$ 
$=0$ and $z=\lambda / 4$ planes. The Courant number versus time for this case is shown in Fig. IV.16. The average Courant number was 10 with peak values of 40-50.

\section{IV.6,4 Resist1ve Plasma Dependence of Growth Rate on S}

A series of cases were $r u n$ in which the only parameter varied was the magnetic Reynolds number. The linear, ideal eigenmodes were used as the initial perturbation $\left(C^{\prime}=10^{-4}\right)$ in our model. The code was allowed to evolve for 5 Alfven transit times in order to allow the Inear mode to become a mode of the nonlinear resistive equations. We, then, restarted the code with a fixed, small $\Delta t(\tilde{\sim} .01)$ and $r$ an for 10 timesteps to calculate the mode's growth rate. The growth rates versus 1/S were plotted in Fig. IV.17. The mode was found to grow faster as the resistivity increased. This is expected since the resistivity now allows the flufd to move across magnetic fleld lines. Thus, the fluid does not have to perform as much work on the magnetic field as in the case of ideal MiD. This was in qualitatlve agreement with the predictions of Ccppi et al. 70

\section{IV.6.5 Resistive Plasma Without Balancing Electric Field and Cooling Terms}

A plasma with $S=250$ was $r$ un whout the external electric field and the cooling belng enabled. As can be seen in Fig. IV.18, spontaneous flows appeared Inmediately as the equilibrium magnetic field began to diffuse and heat the plasma. This spontaneous flow completely masked the initial perturbation on the velocity field 
diagnostics; but the growth of the $1 / 1$ mode was evident in the Fourier analyzed energy plots (Fig. IV.19). The $1 / 1$ mode reappeared in the velocity fields only after it has grown by 2 orders of magnitude and began to dominate the spontaneous flows (Fig. IV. 20).

\section{7 Summa ry}

We have shown agreement with Bateman ${ }^{9}$ and $\mathrm{H}: \mathrm{cl} \mathrm{r}^{55}$ both with the form of the linear ideal eigenfunctions and with their growth rates. The addition of resistivity was shown to enhance the ideal growth rates without changing appreciably the form of the eigenfunctions. A simulation which extended for 90 Alfven transit times was presented which displayed a new plasma state that was characterized by only an extremely slowly growing 3,1 mode. Finally, the terms added to balance the effects of finite resistivity were shown not to affect the calculated growth rates.

All the above simulations were performed at Courant numbers substantially greater than unity. 


\section{$-108-$}

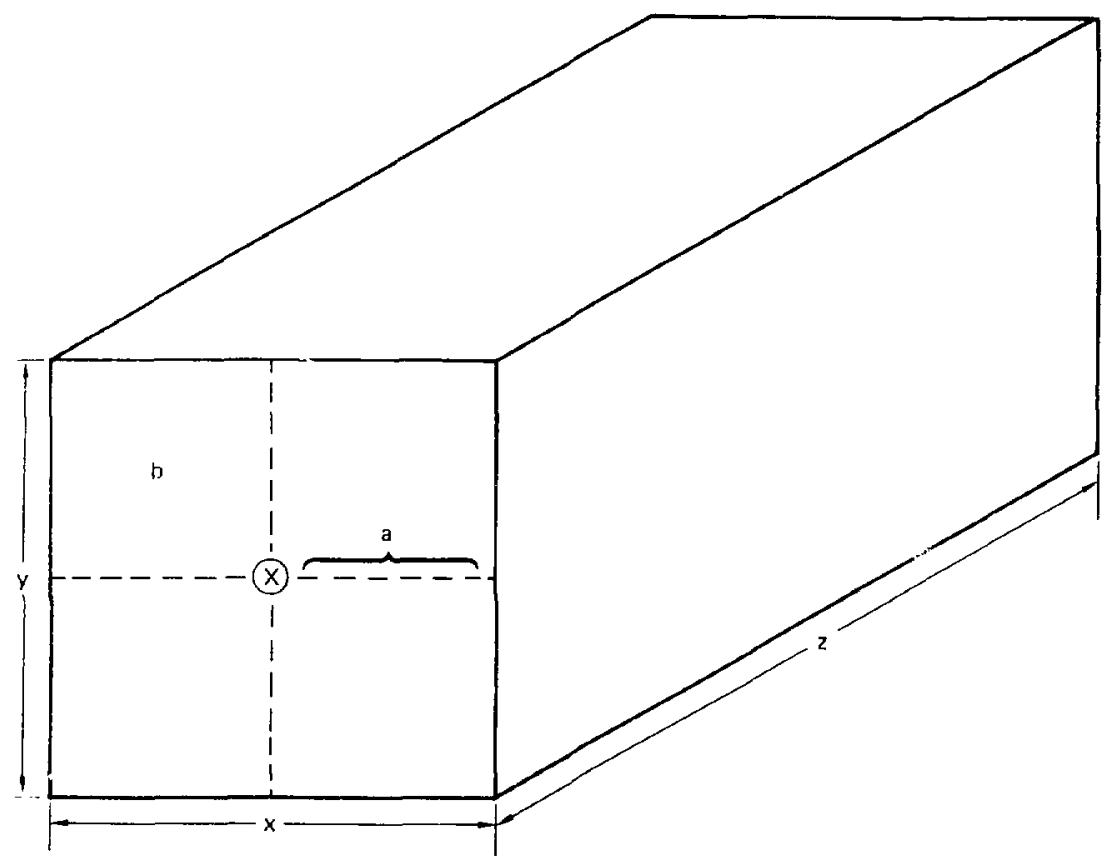

Figure IV.1 The computational region used in Cartesian coordinates. The origin is at $\mathrm{x}$ and $\mathrm{b} / \mathrm{a}$ is the squareness ratio. 


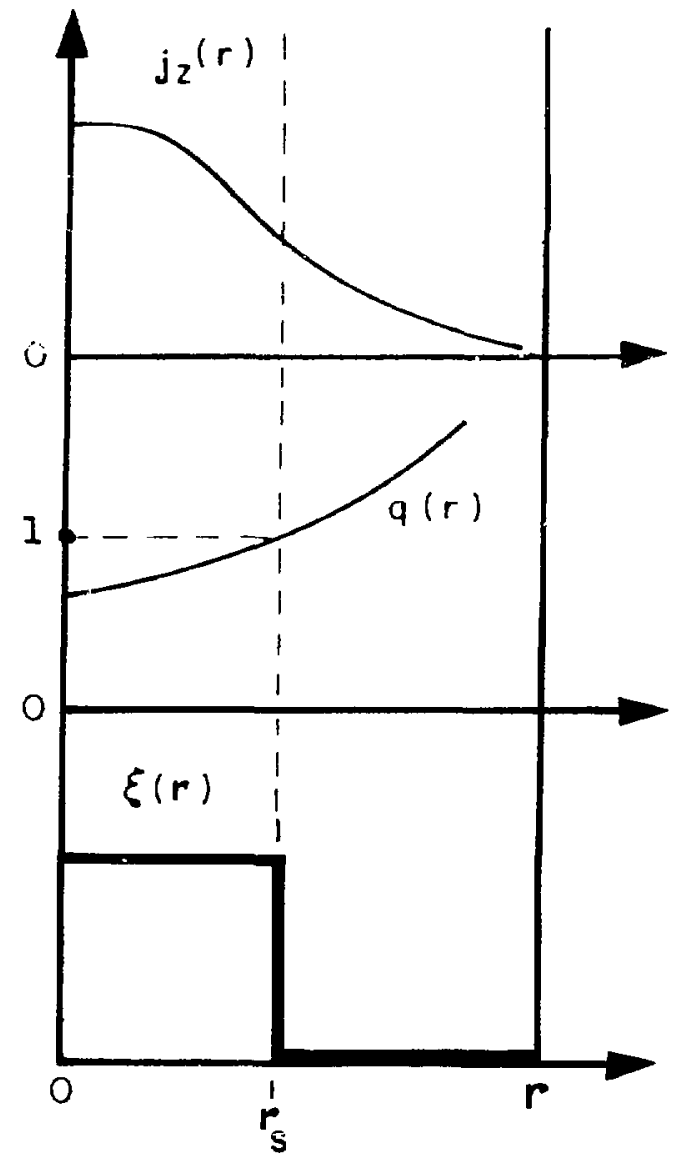
Figure IV.2 Radidl profiles of the linear eigenfunctions of current
$j_{(}(r)$, safety faclor $q(v)$ and radial displacement $\xi(r)$. The singi-ar surface $\left(k . \underline{B}_{-}=0\right)$ is at $r_{s}$. 


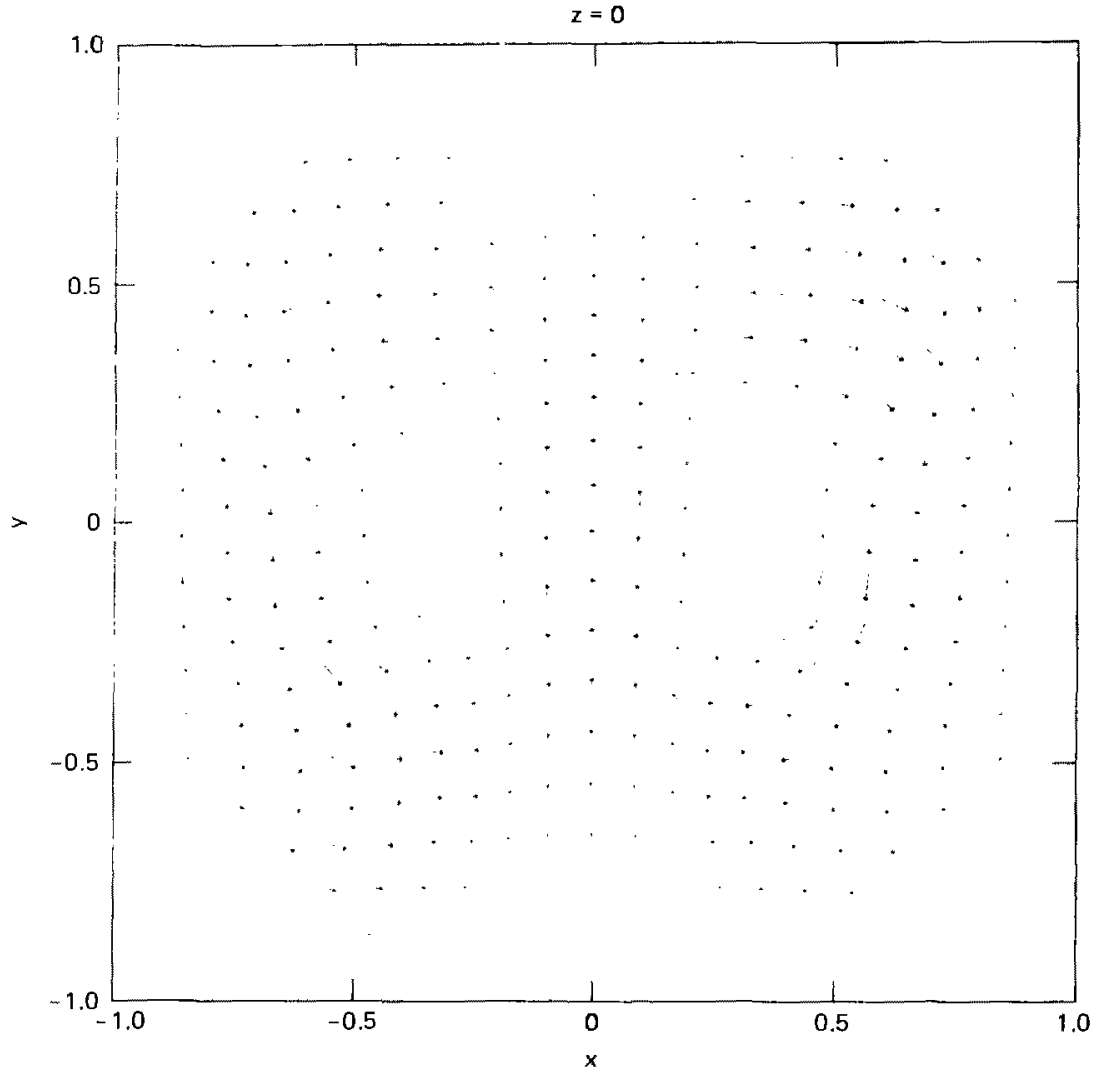

Maximum velocity in plane (arrows) $=1.04097633 E \cdot 04$

Maximum perpendicular velocity (circles) $=0$.

Elgure IV.3a the initial velocity field used to perturb the plasma on the $z=0$ plane. The length of each arrow is proportional to the relative velocity at that point, normalized to the maximum velocity on the plane. 


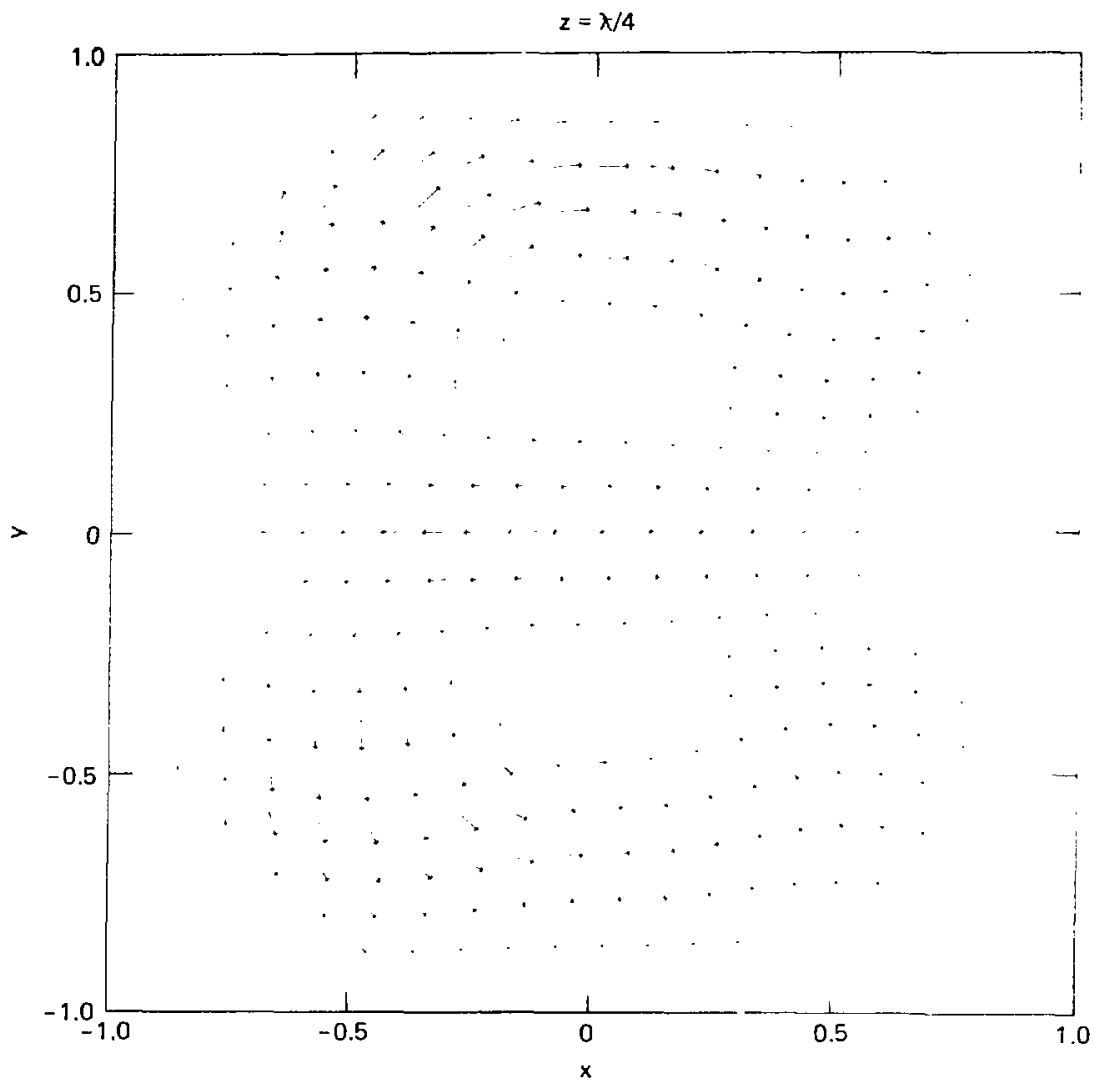

Maximum velocity in plane (arrows) $=1.04037633 E .04$

Maximum perpendicular velocity (circles) $=0$.

Figure LV.3b The initial velocity perturbation on the $z=\pi / 4 \mathrm{plane}$. 


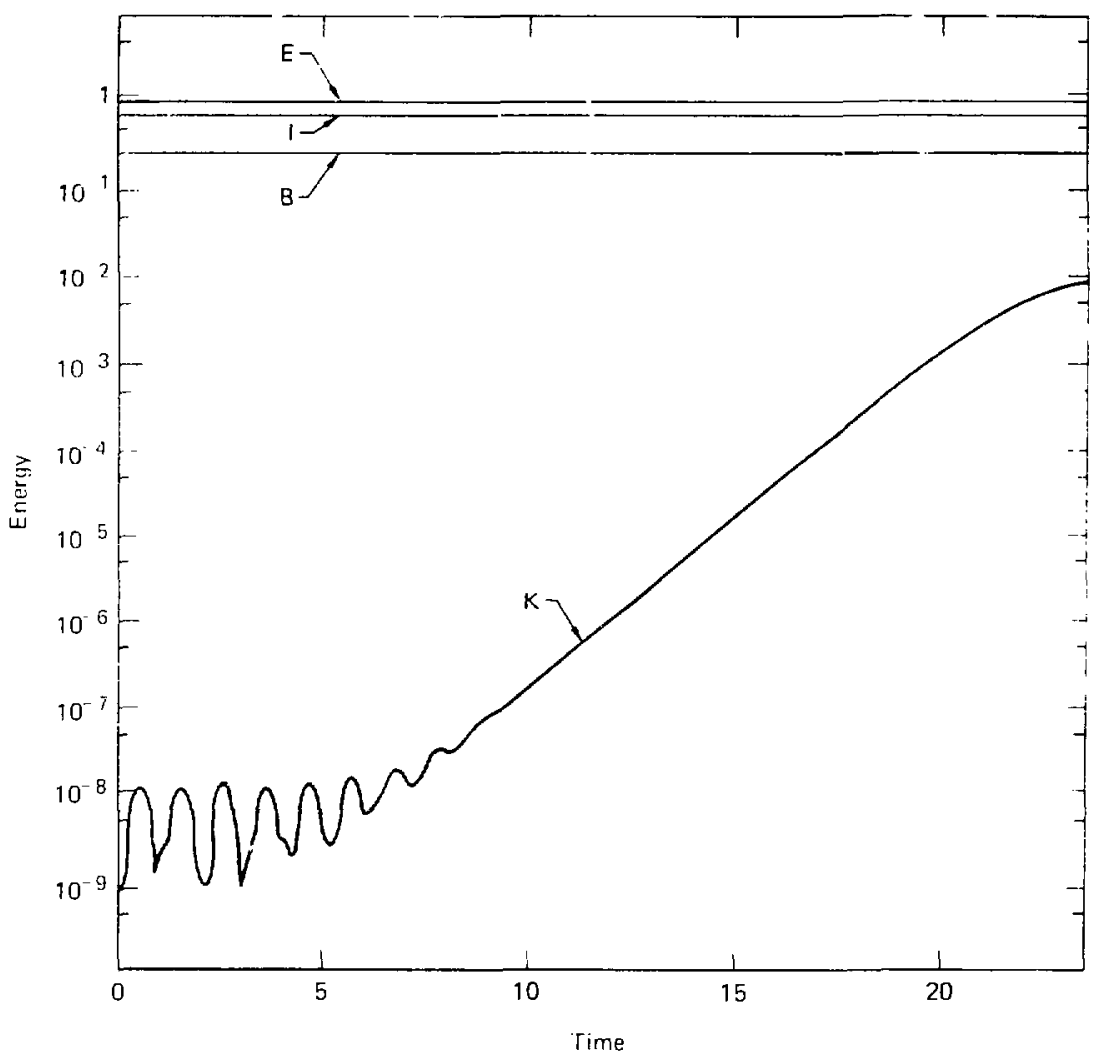

Figure IV.4

The various components of the energy summed across the entire mesh are plotted as functions of time. The trace labeled $i s$ is the kinetic energy, the B trace is the ma pnelic energy, the I trace is the internal enersy, and tie F crace is the cotal energy. 


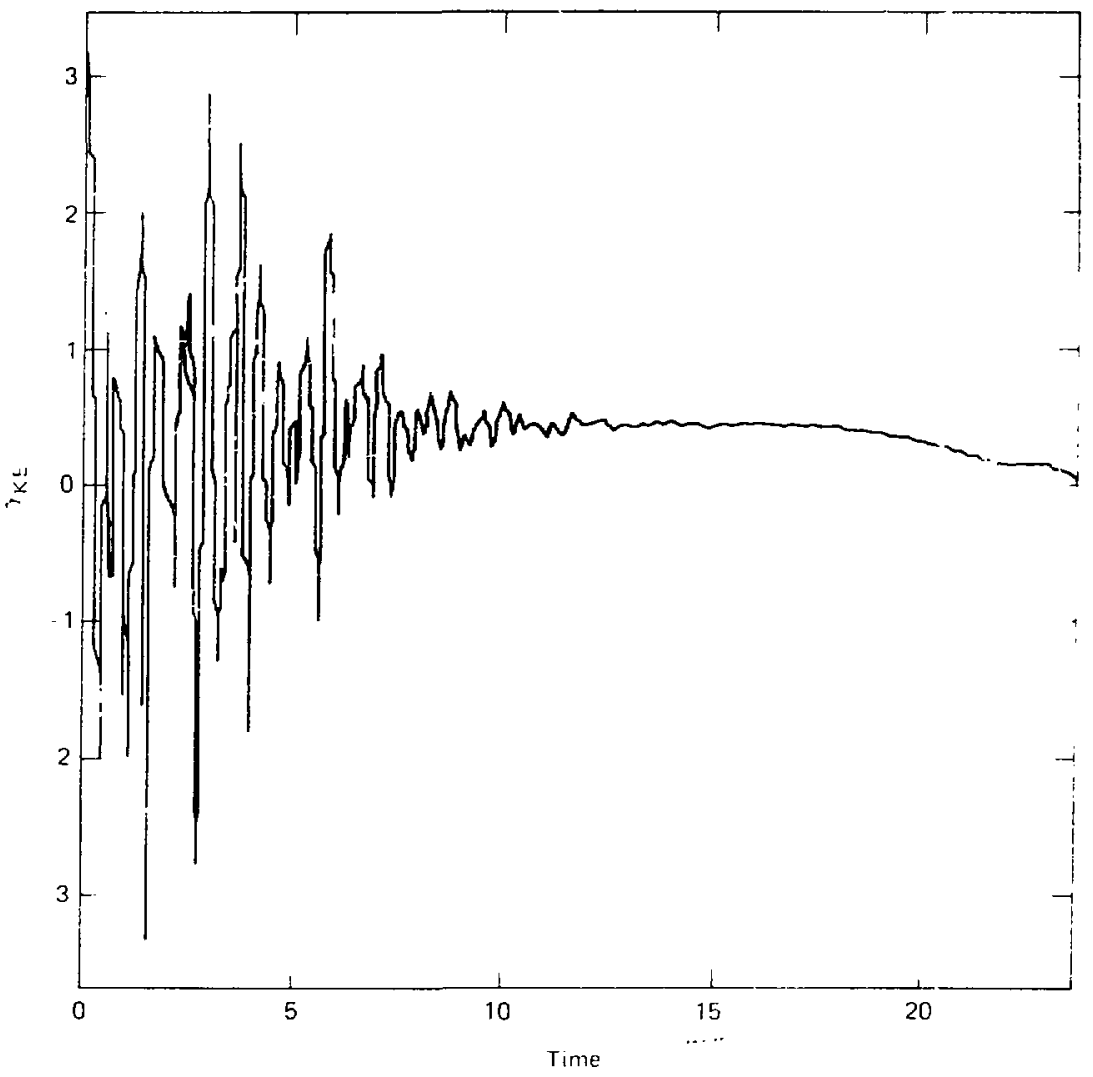

Fig.re IV.5 The growch rate based on kinetic energy $\because r^{2}=\frac{1}{2} \frac{d\left(n_{i}\right)}{d t}$ plotted as it function of time. The inteial oscillaticns are followed by a period of exponential wrowth which then ceases as nonlinear saturation occurs. 


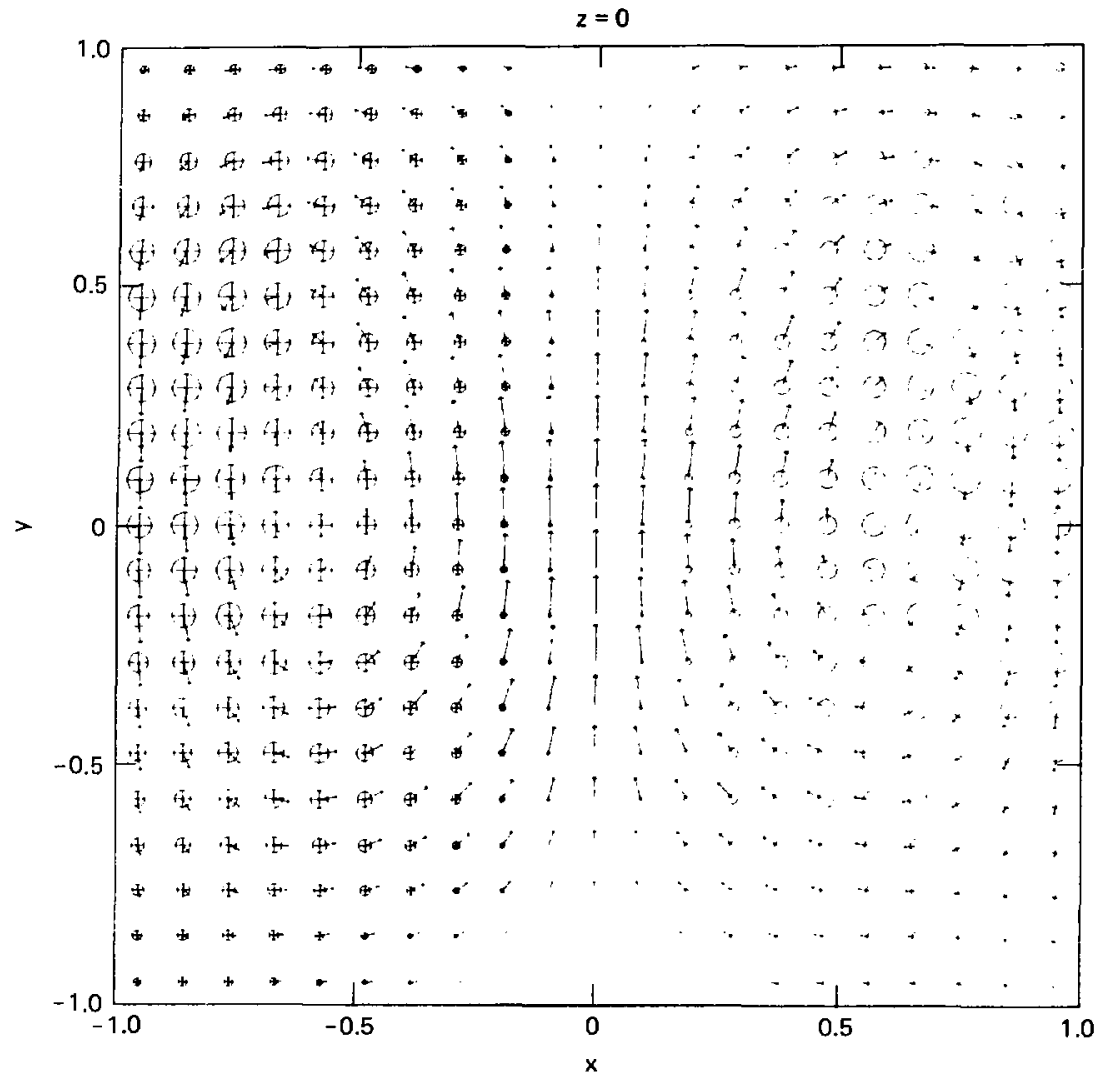

Maximum velocity in plane (arrows) $=2.33030233 \mathrm{E}-01$

Maximum perpendicular velocity (circles) $=1.74644917 \mathrm{E} 0-1$

Figure I.6a

The velocity field on the $z=0$ plane during the exponential growth phase. The velocity in the $z$ direction is represented by the circles. The magnitude of the $z$ velocity is proportional to the radius of the circle while the direction of the velocity is indicated by the presence of a cross inside the clrcle (negative $v_{z}$ ) or its absence (positive $v_{z}$ ). 


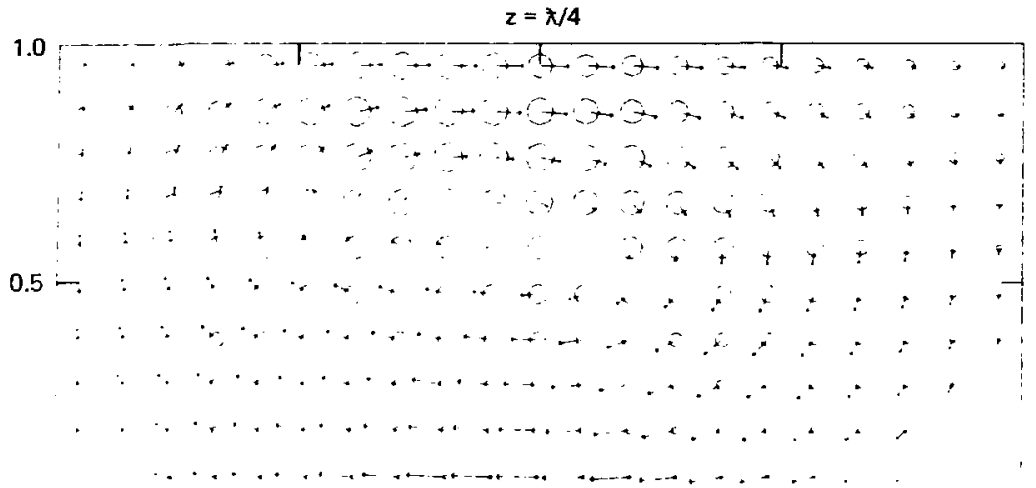

$>\quad 0-$

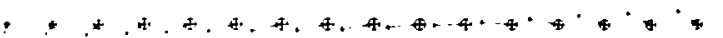

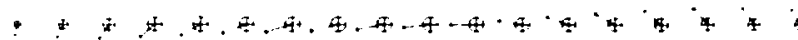

-0.5 -

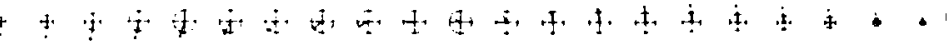

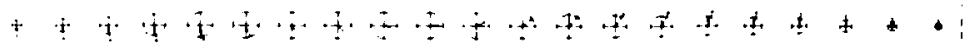

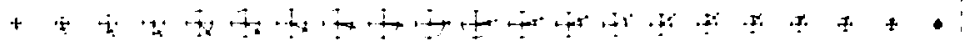

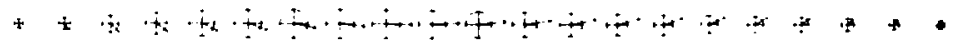

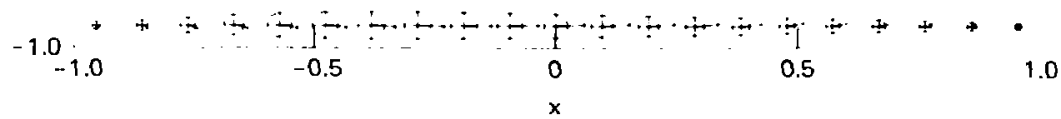

Maximum velocity in plane (arrows) $=2.32781400 \mathrm{E}-01$

Maximum perpendicular velocity (circles) $=1.79158183 \mathrm{E}-01$

Figure iV.6b The velocity field on the $z=\lambda / 4$ plane during the exponential growth phase. 
$-116-$
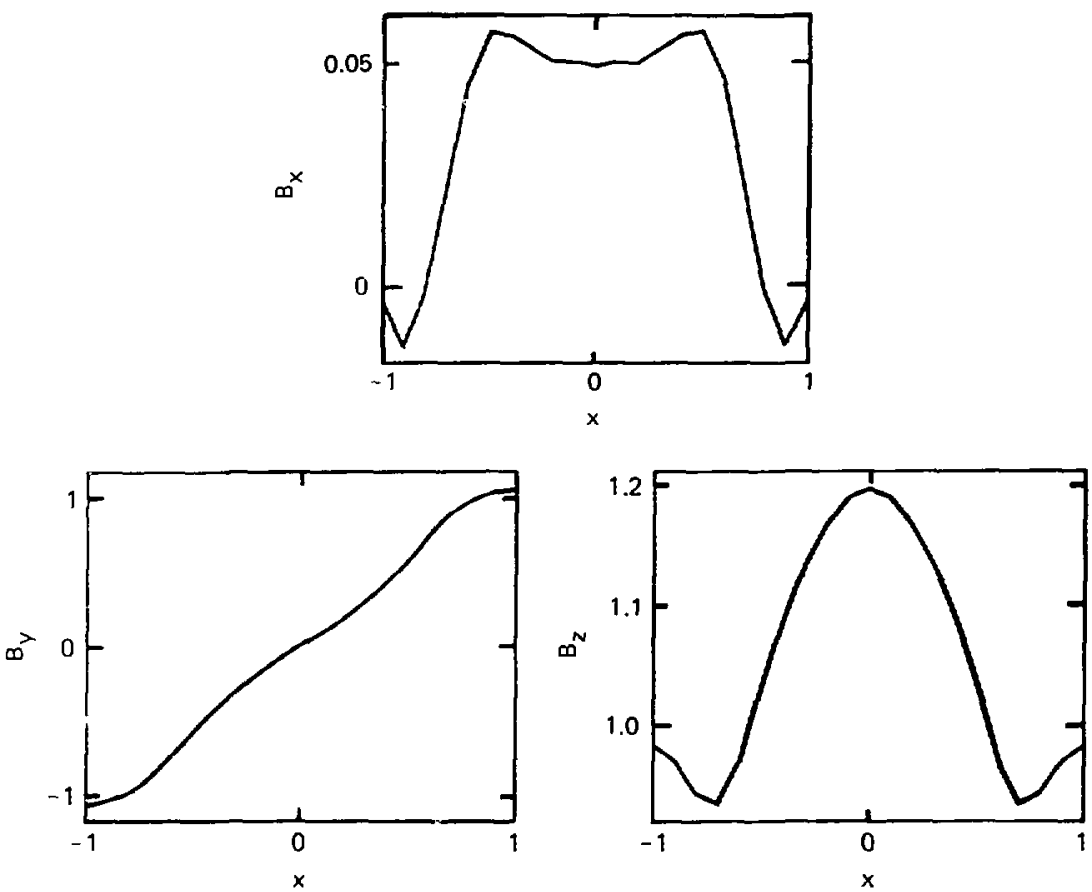

Figure IV.7a The state variables along a 1 ine $-1 \leq x \leq 1, y=0, z=0$. 

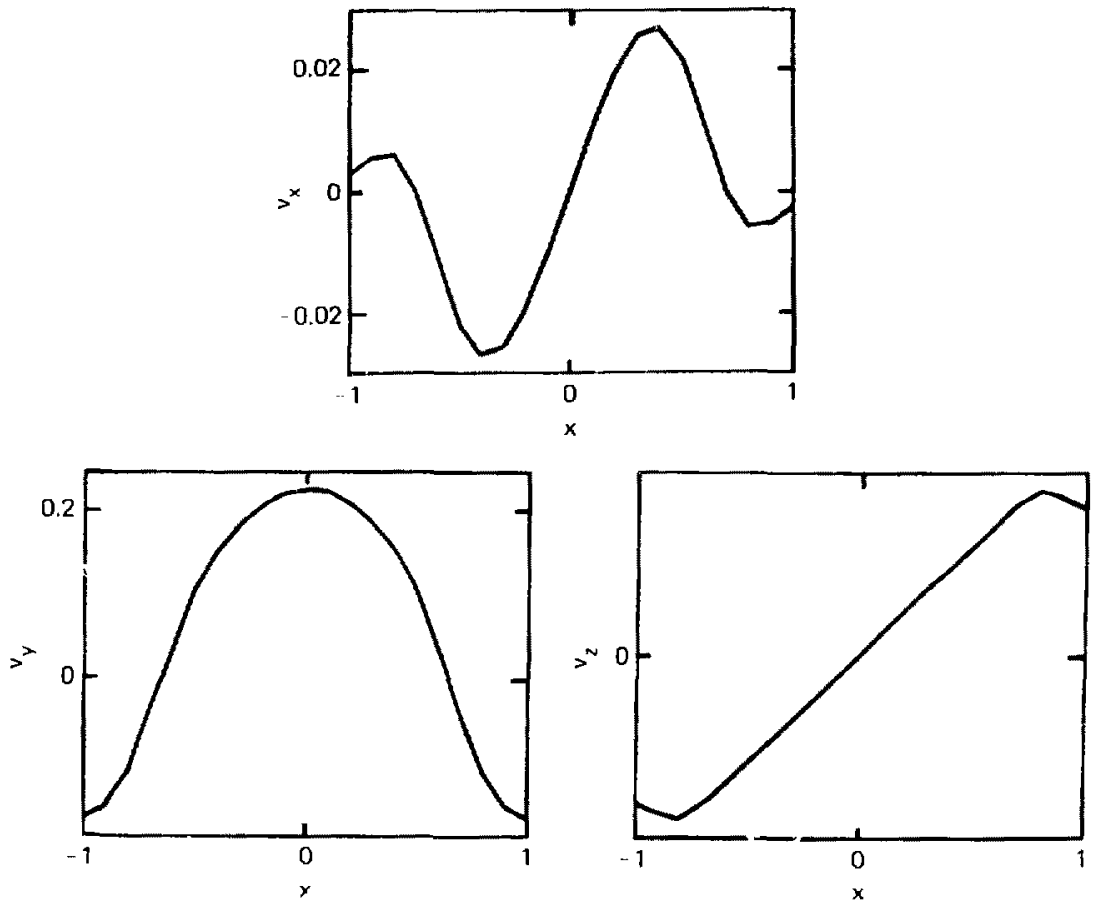

Figure $[\because .7 b$ The state variables along a line $-1 \leq x<1, y=0, z=0$. 

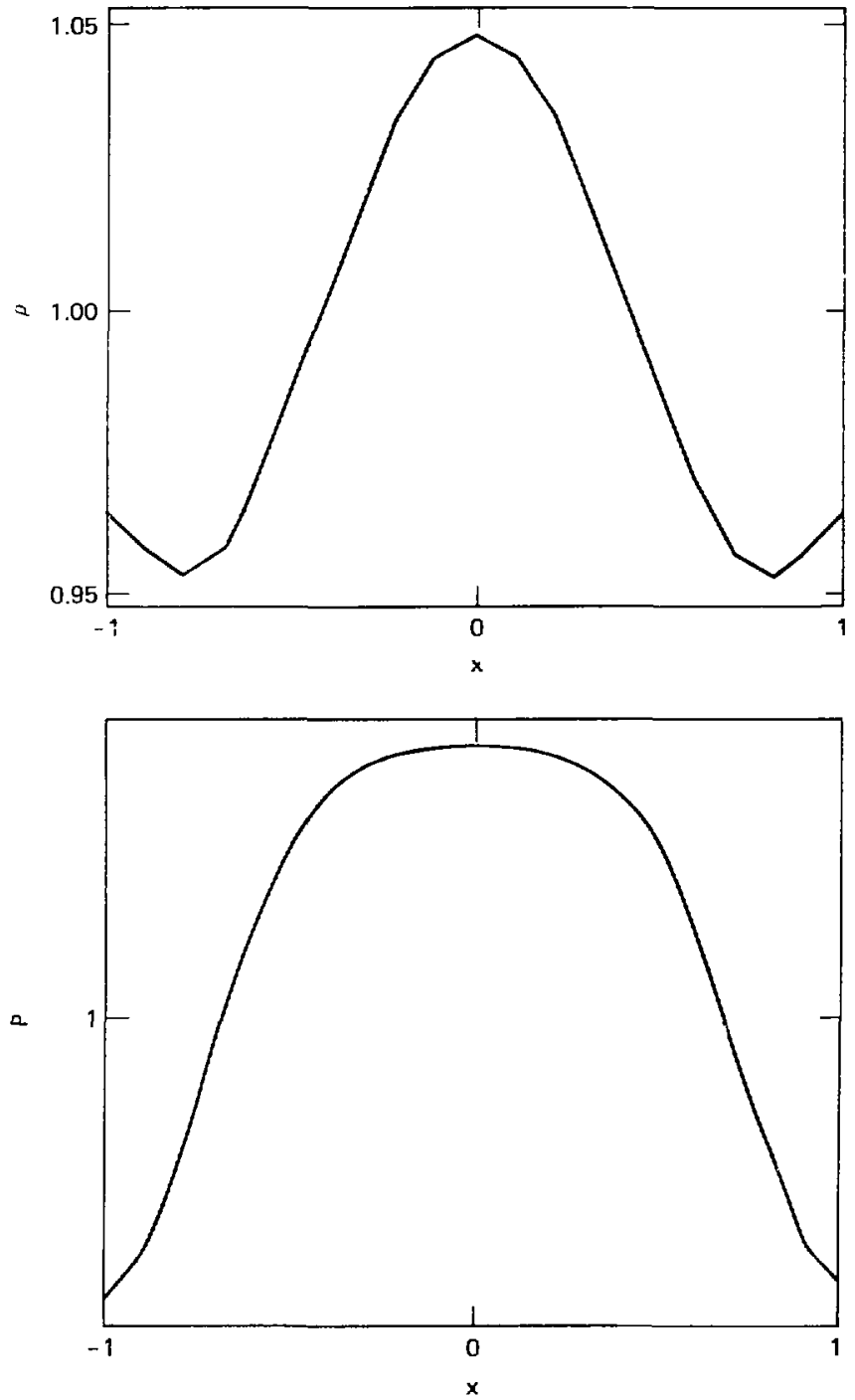

Figure IV.7c The state variables along a line $-1 \leq x \leq 1, y=0, z=0$. 


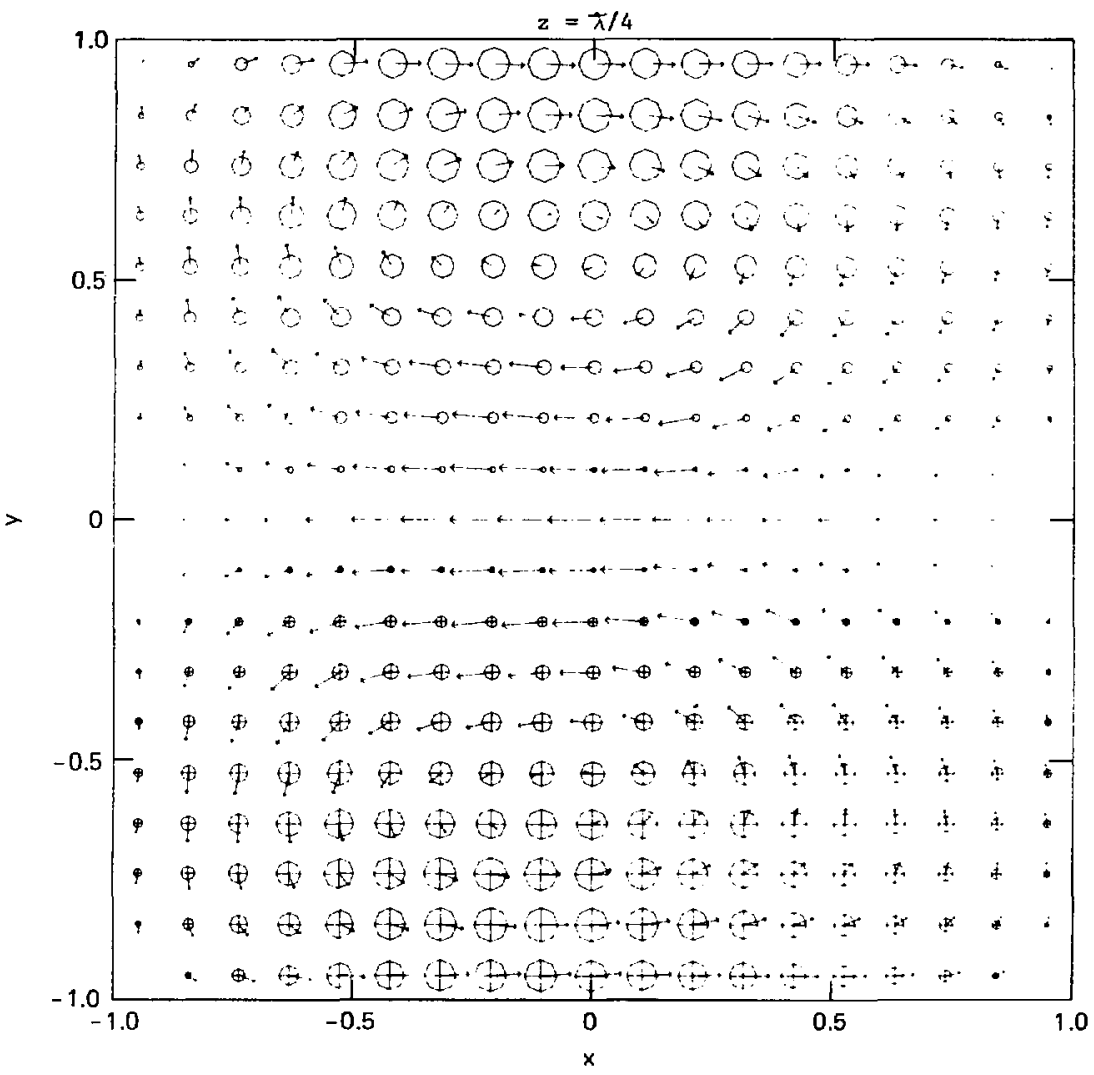

Maximum velocity in plane (arrows) $=2.22039553 \mathrm{E} \cdot 01$

Maximum perpendicular velocity (circles) $=1.48435383 \mathrm{E}-01$

Figure IV.8 The velocity field at the onset of saturation of the 1/1. mode on the $z=\pi / 4$ plane. 
1.0

$z=\pi / 4$

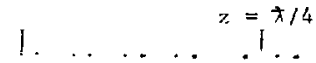

$0.5-$

$>\quad 0-$

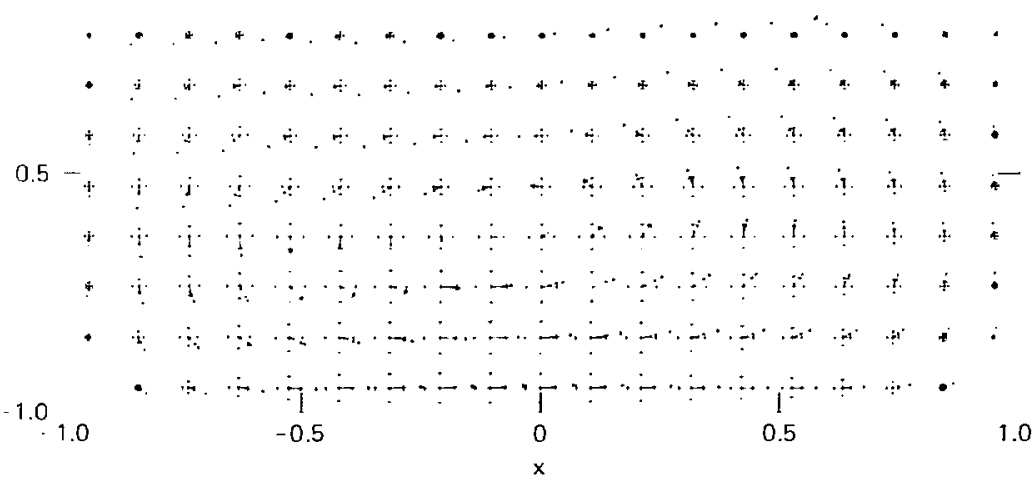

Maximum velocity in plane (arrows) $=3.06709026 \mathrm{E}-01$

Maximum perpendicular velocity (circles) $=2.44027112 \mathrm{E} \cdot 01$

Figure IV.9 The velocity field at saturation on the $z=9 / 4$ plane. 
$z=0$

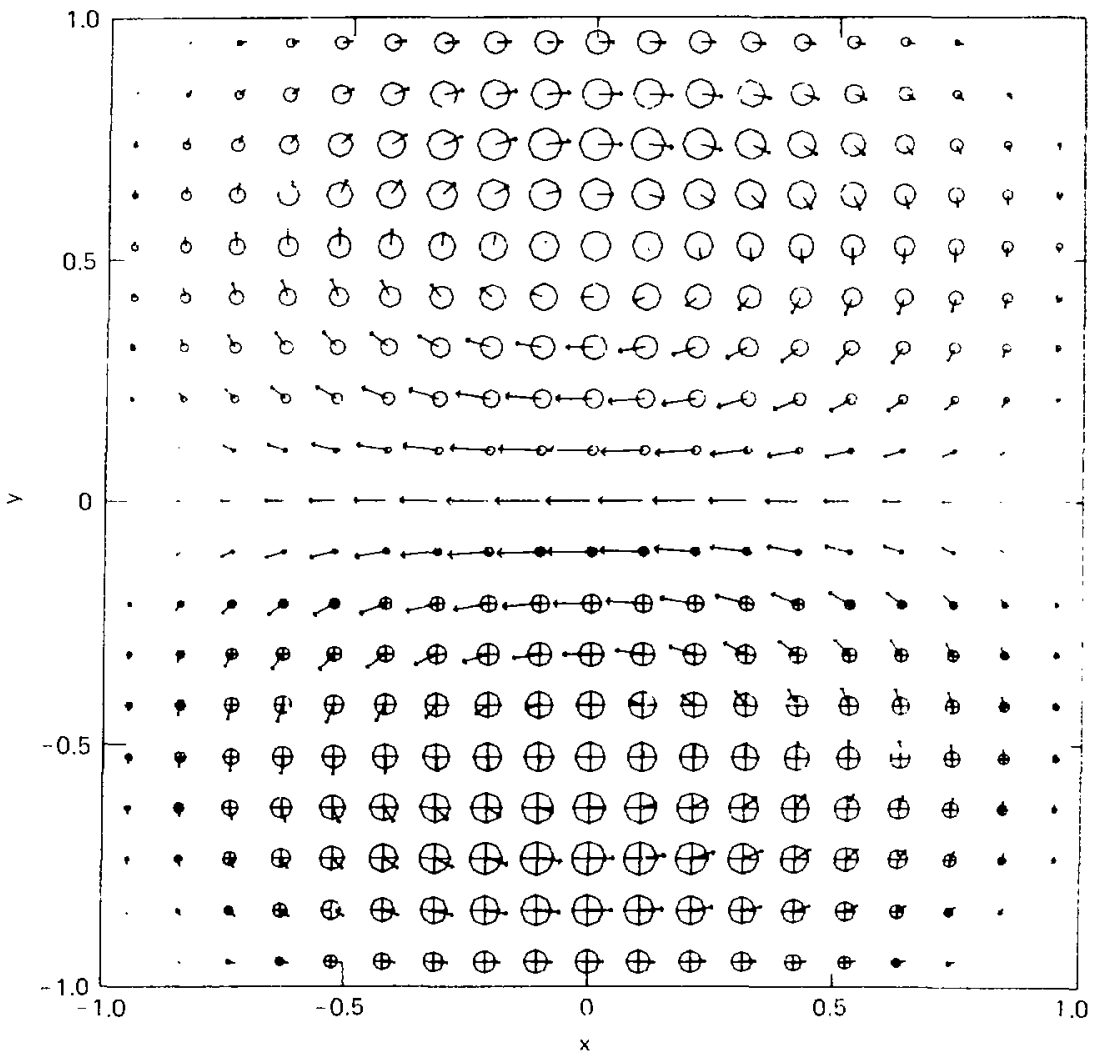

Maximum velocity in plane (arrows) $=9.92154827 \mathrm{E} \cdot 05$

Maximum perpendicular velocity $($ circles $)=4.61674548 \mathrm{E}-05$

Figure IV.10a The velocity eigenfunction projected (n) the $z=$ li plane. 
$z=\pi / 4$

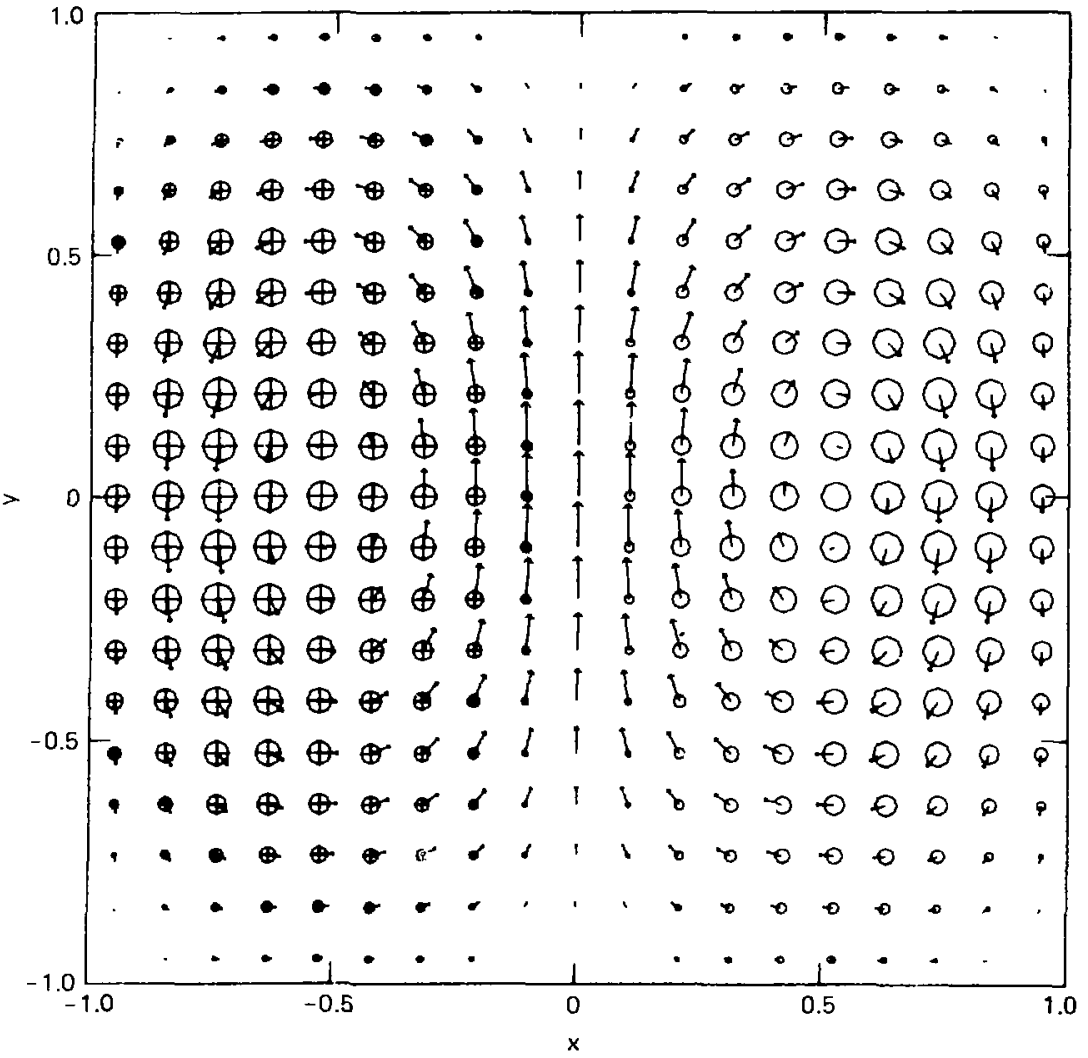

Maximum velocity in plane (arrows) $=1.00000000 E-04$

Maximum perpendicular velocity (circles) $=4.67603787 \mathrm{E}-05$

Figure IV.10b The velocity eigenfunction projected on the $z=\pi / 4$ plane. Nate the comparison of these velocity fields with the veloctty fields found by InP piven in Figure $1 \mathrm{~V}-6 \mathrm{a}$ and $\mathrm{b}$. 


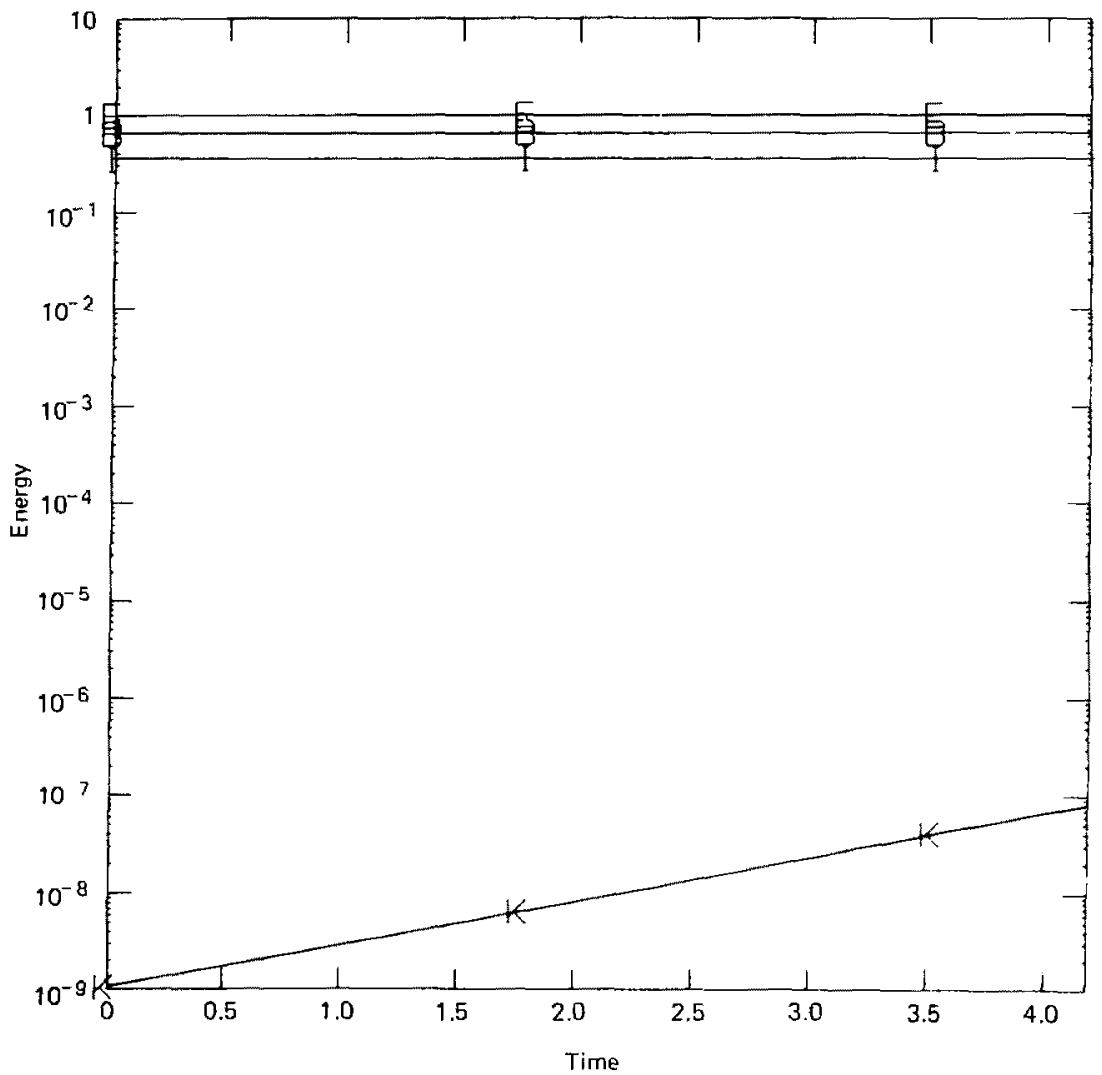

Figure IV.11a The energy components plotted versus time for the initial perturbation by linear eigenfuncticns. The kinetic energy displays immediate exponential growth. 


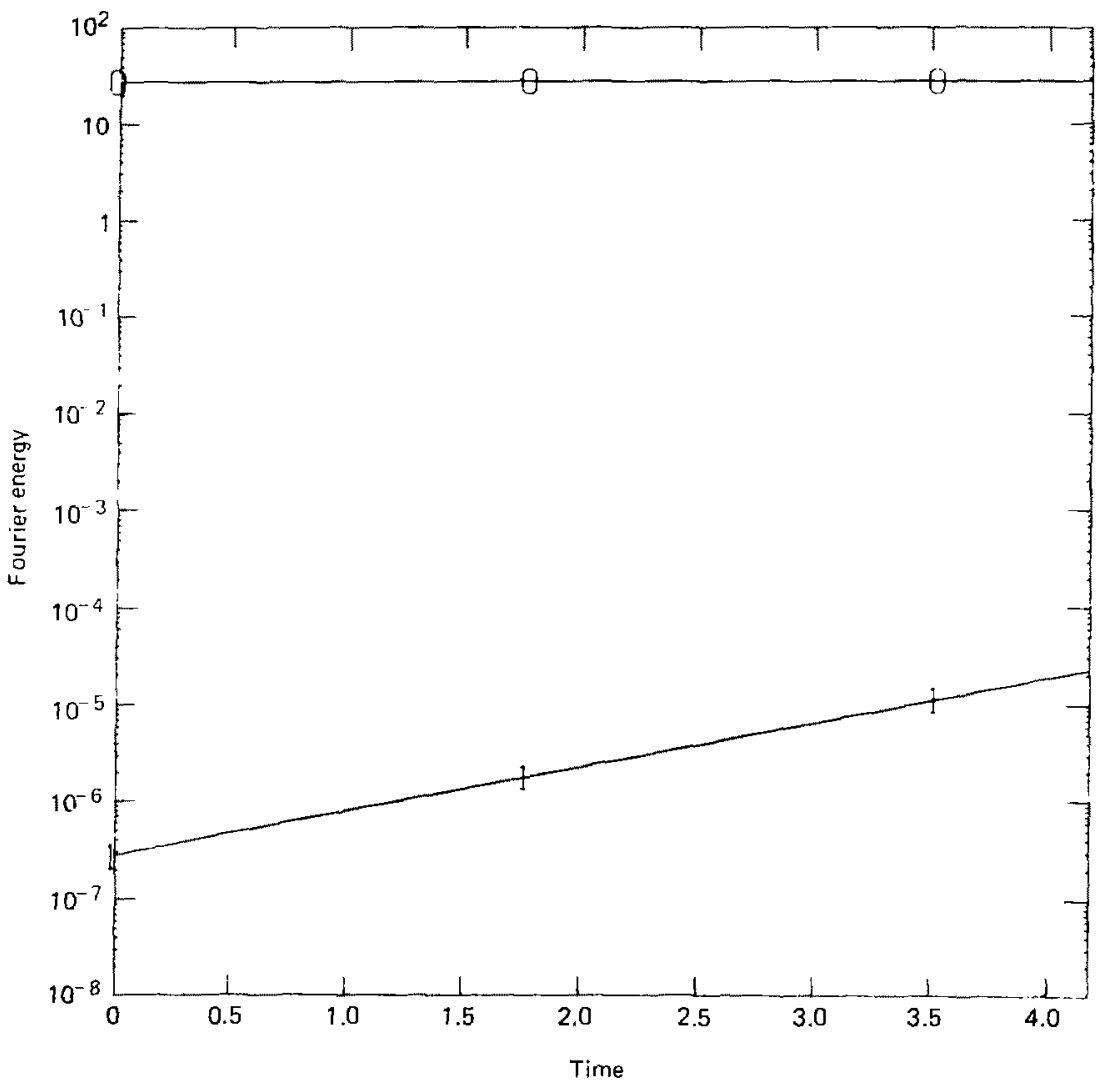

Tinure IV.1Ib The energy contained the $n=0$ and $n=1$ Fouriet modes. Here again immediate exponential growth of an $n=1 \mathrm{mod}$. can be observed. 


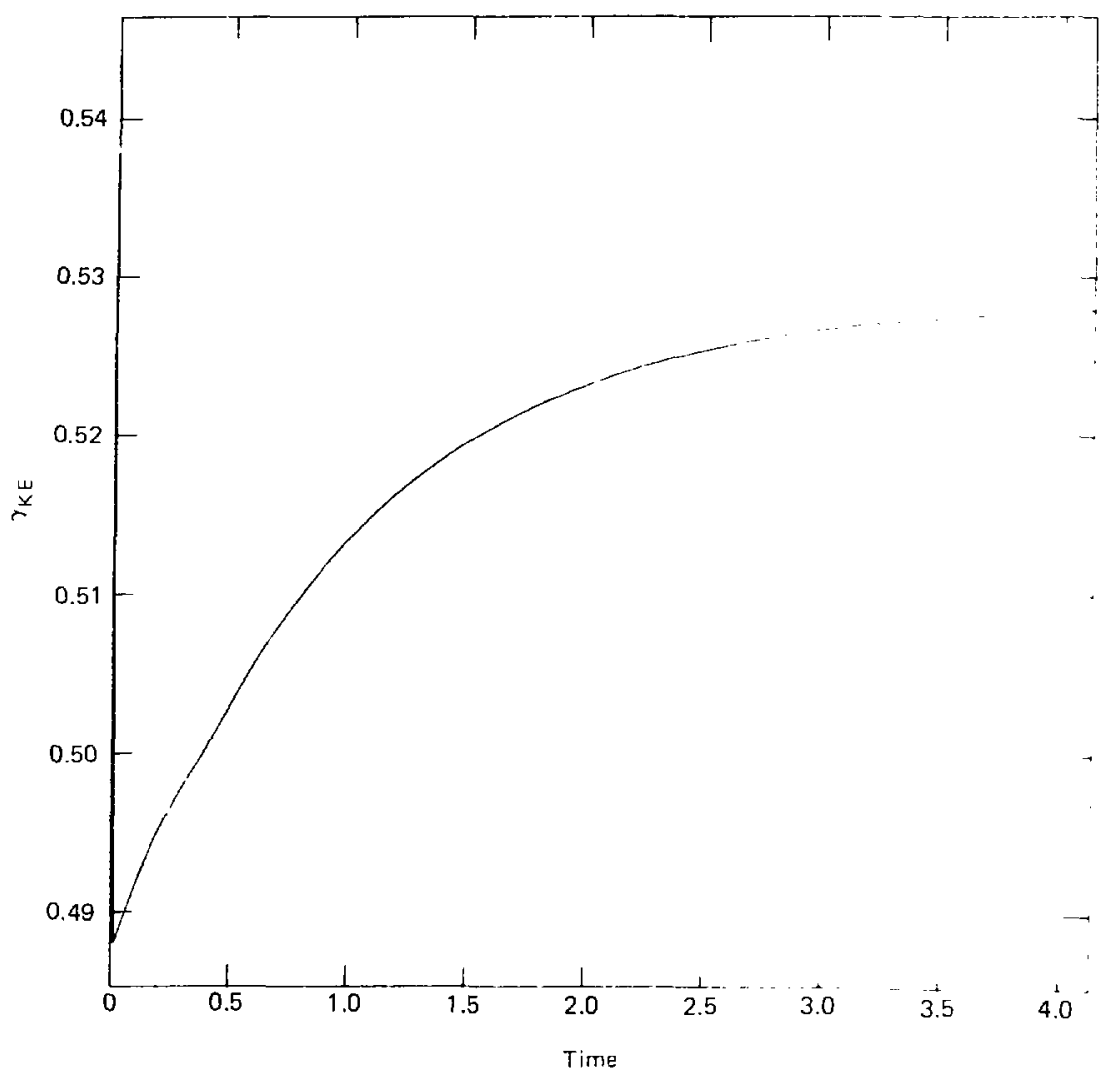

Figure IV.12a The growth rate based on the kinetic energy versus time. 


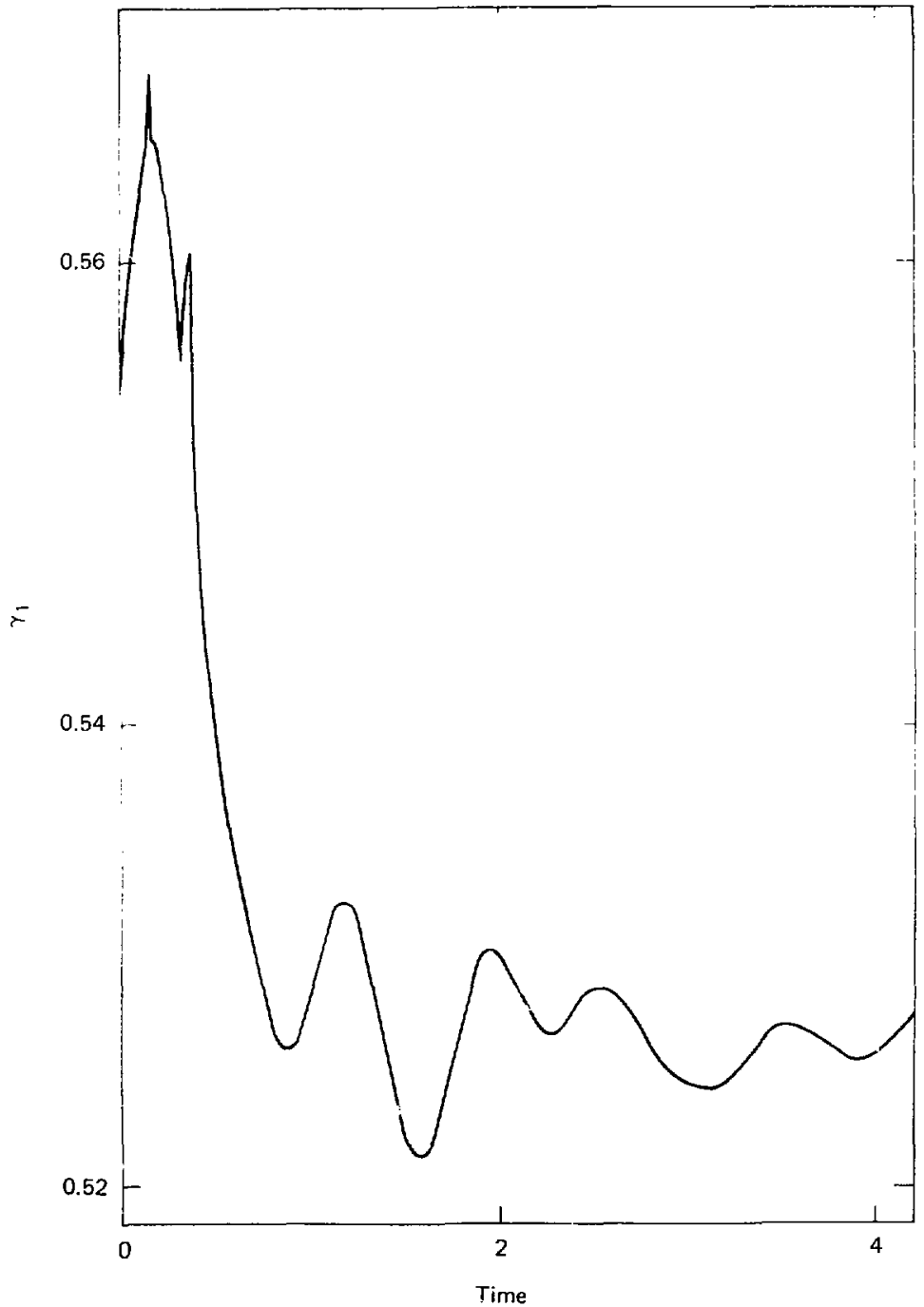

Figure IV.1 $1 \mathrm{~b}$ The growth rate based on the $\mathrm{n}=1$ Fourier mode displayed versus time. 


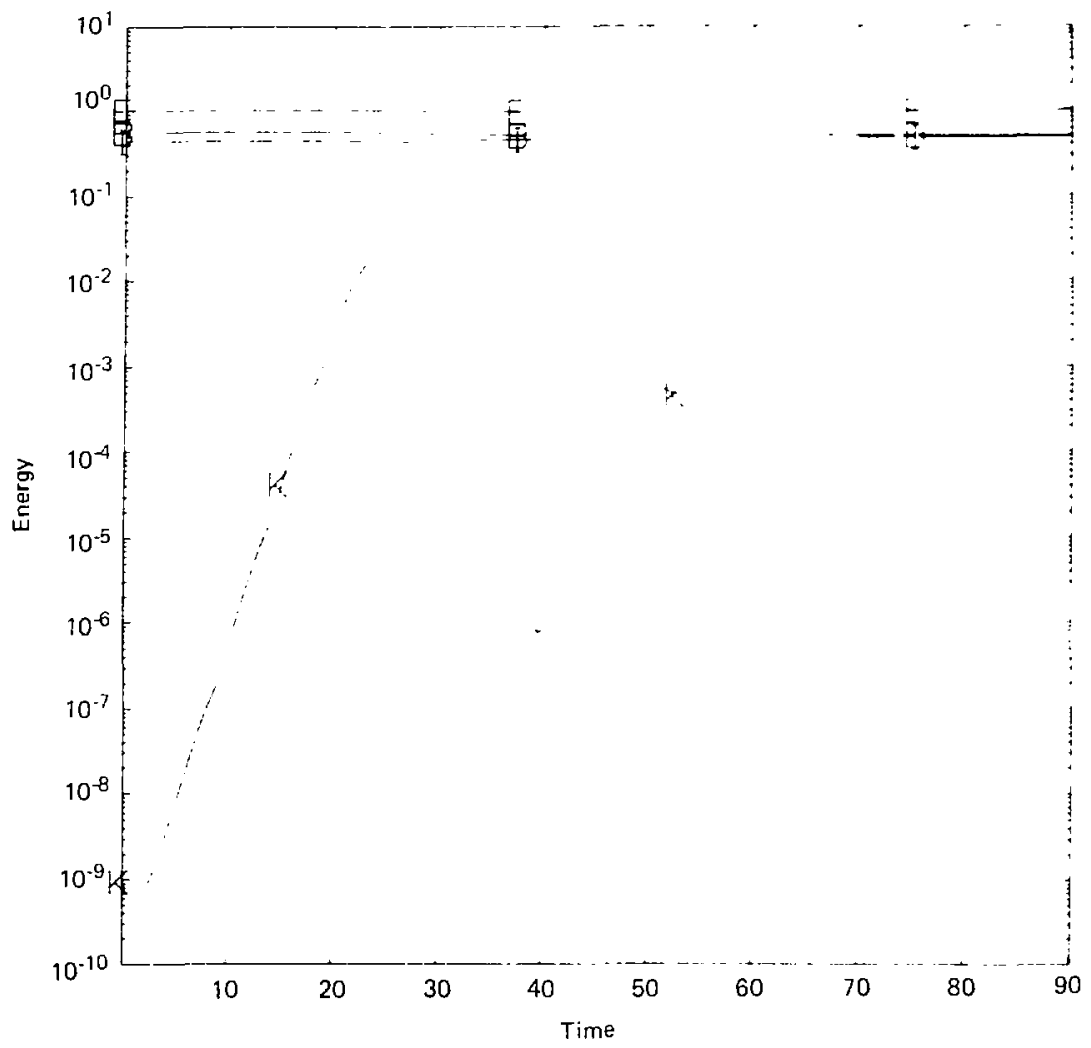

Figure IV.13a The kinetic energy plotted versus time for the long time resistive simulation. 


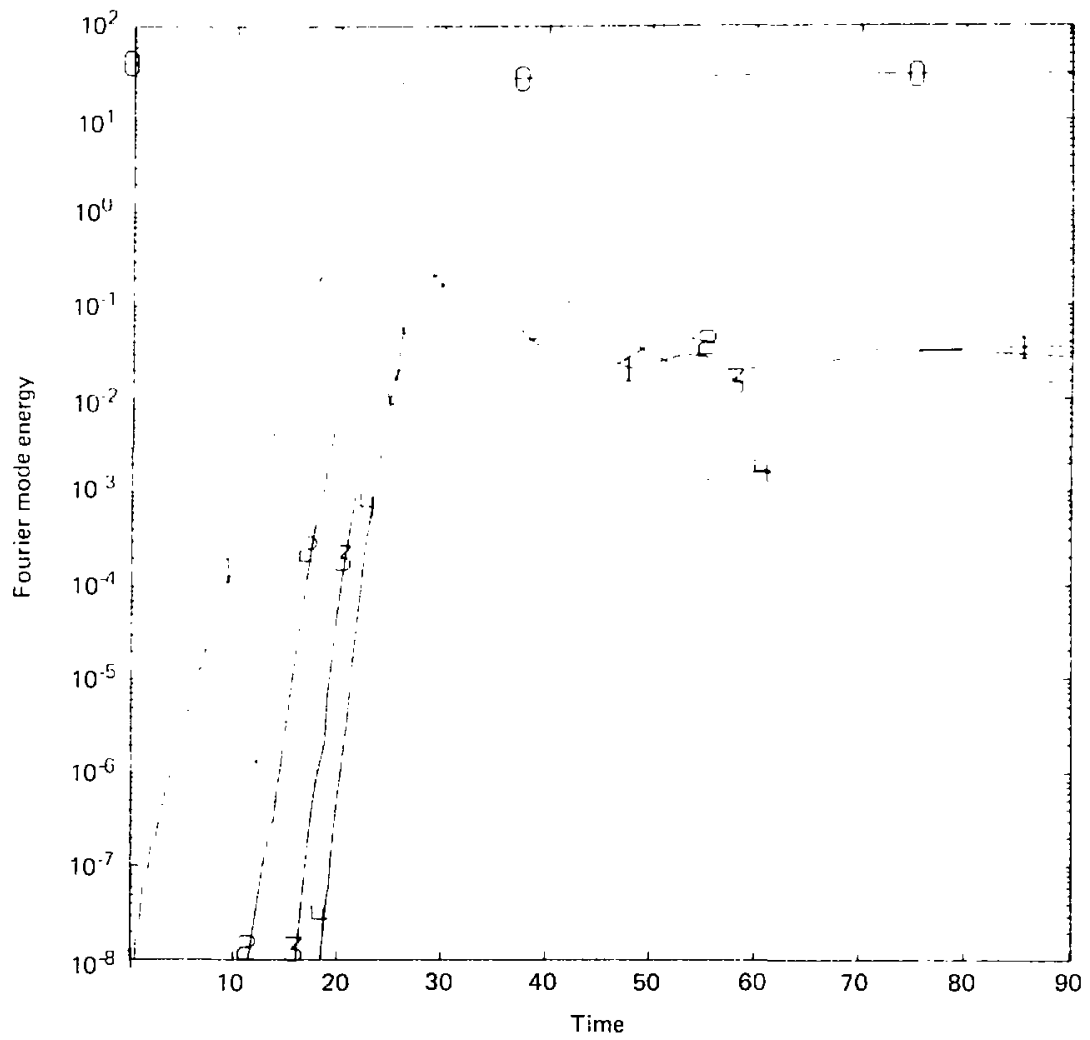

Figure IV.13b The Fourier mode energies versis =ime for the long t1me resistive simulation. 


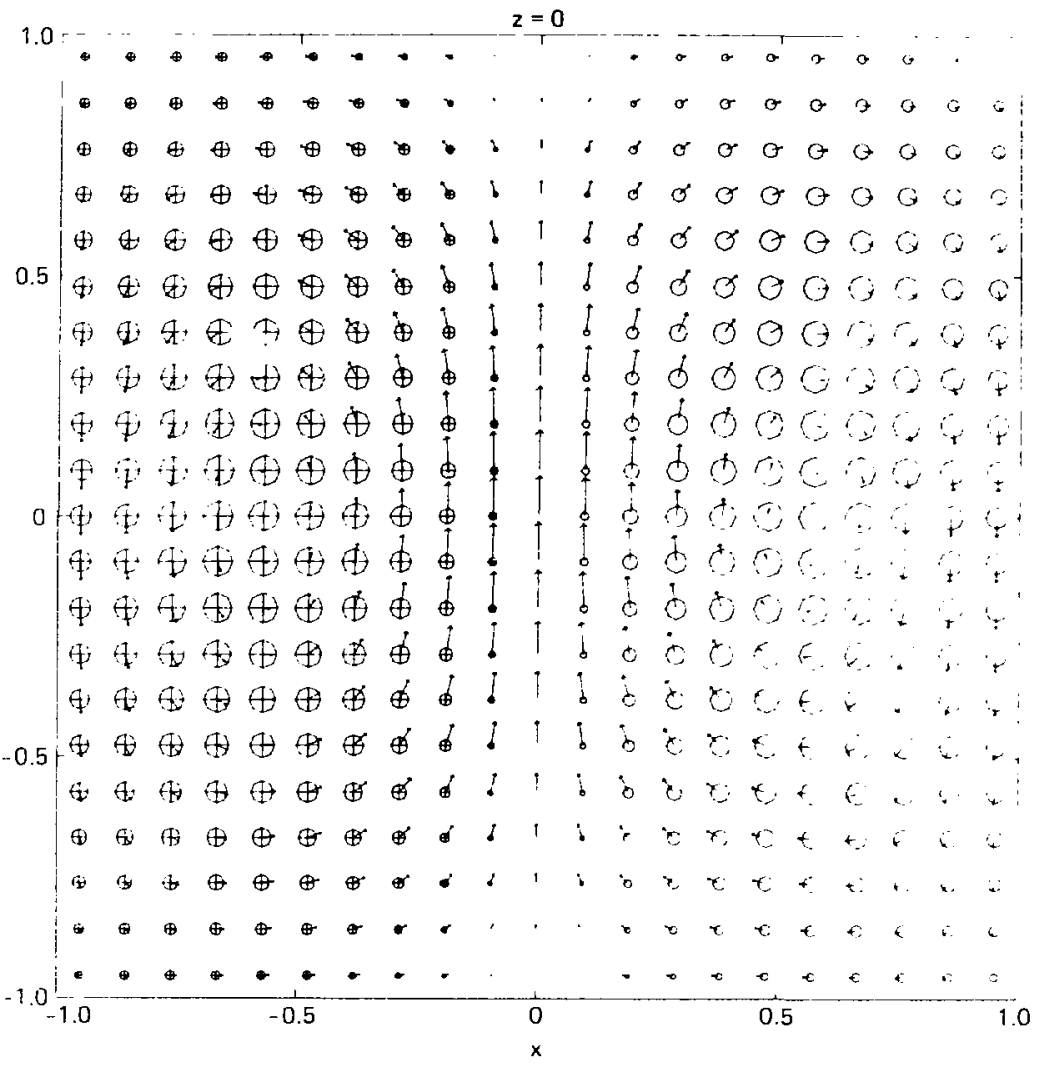

Maximum velocity in plane (arrows) $=1.88232761 E \cdot 03$

Maximum perpendicular velocity (circles) $=378616857 \mathrm{E}-04$

Figure IV.14a The velocity field on the $z=0$ plane during the exponential growth phase of the resistive simulation. 


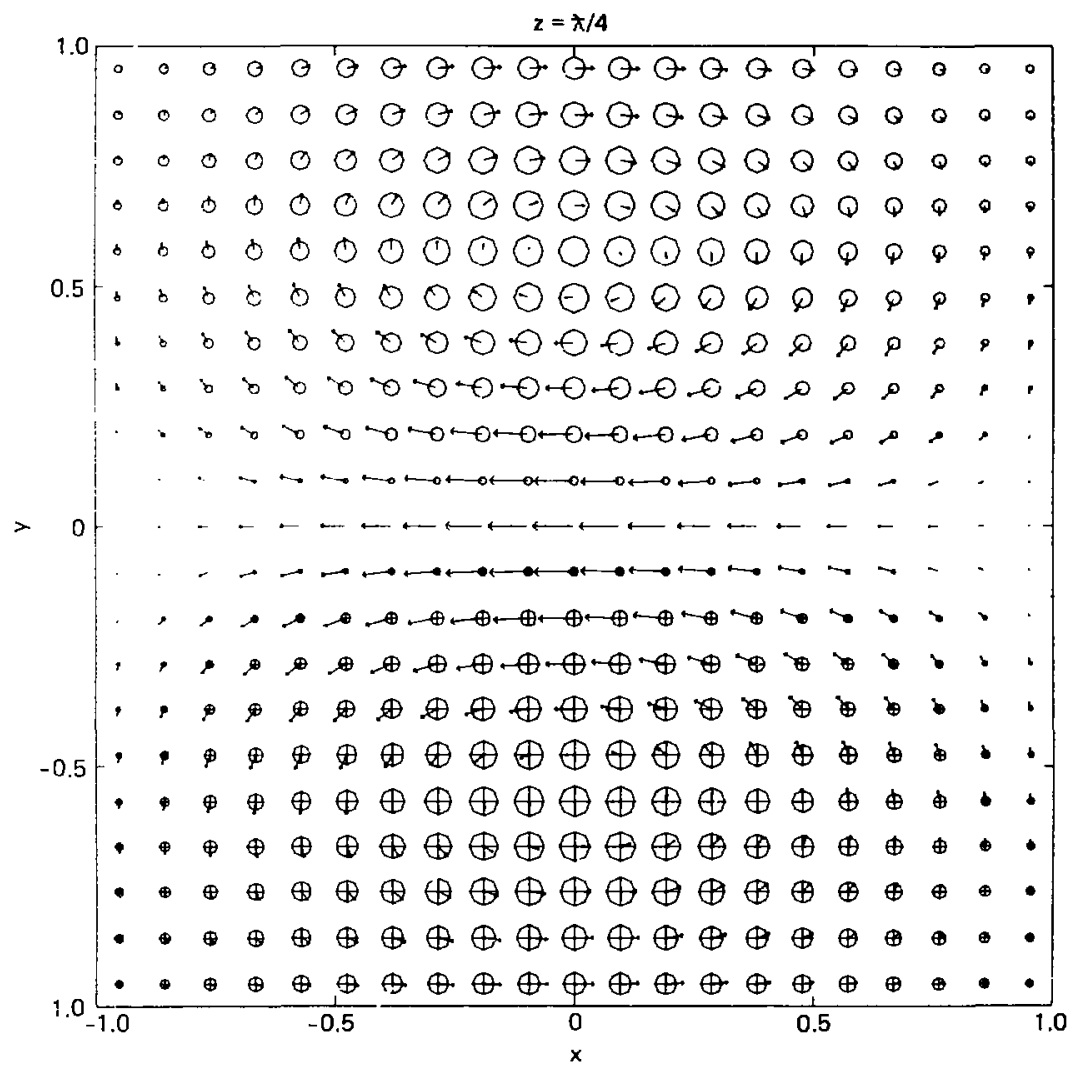

Maximum velocity in plane (arrows) $=1.93540822 \mathrm{E} \cdot 03$

Maximum perpendicular velocity (circles) $=9.87186103 \mathrm{E}-04$

Figure IV.14b The velocity fuelds on the $z=\pi / 4$ plane. 


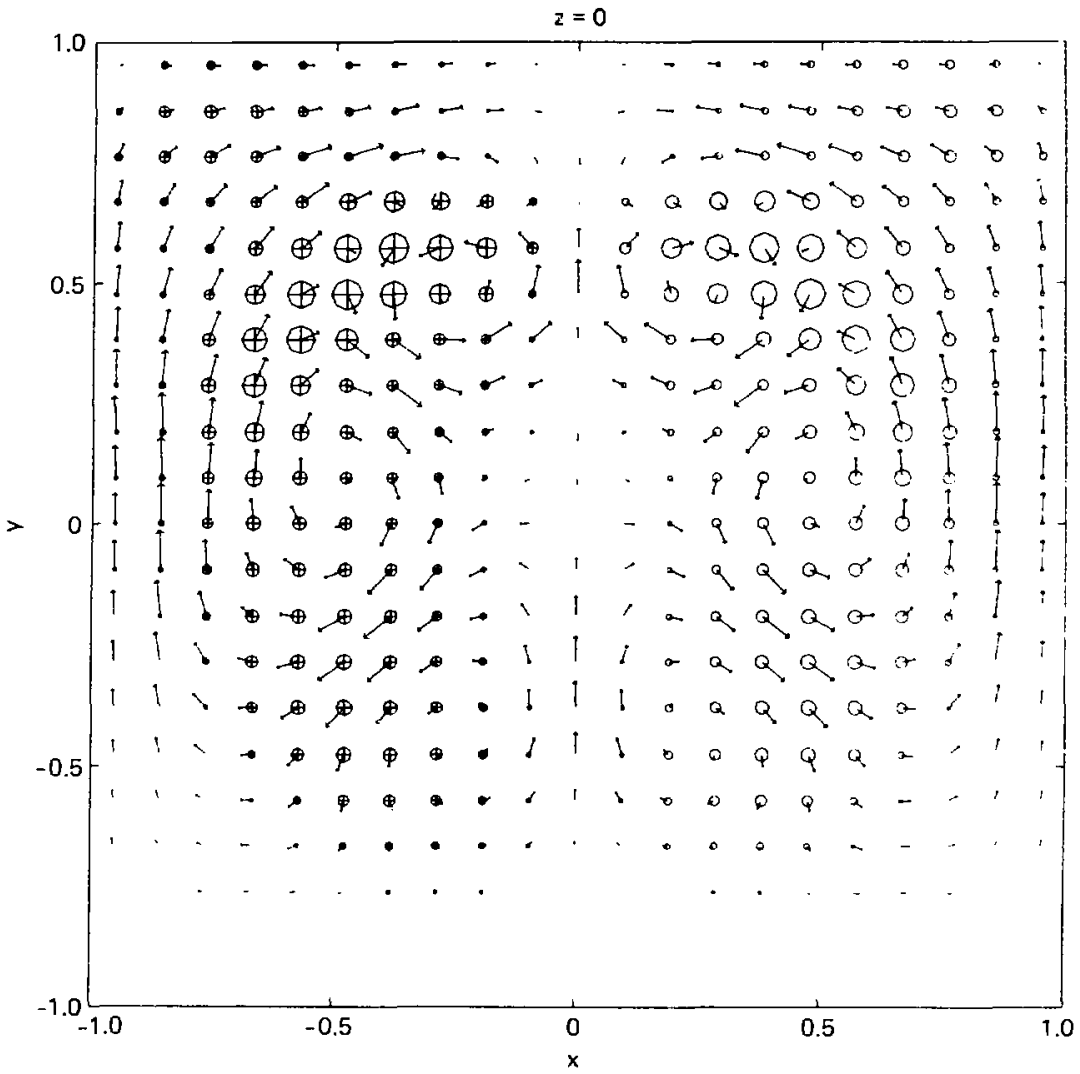

Maximum velacity in plane (arrows) $=4.56829917 \mathrm{E}-02$

Maximum perpendicular velocity (circles) $=5.73983576 \mathrm{E}-02$

Figure IV.15a The velocity field at $t=90$ on the $z=0$ plane. The $m=3$ character of the mode can be seen by the presence of 6 velocity vortics. 


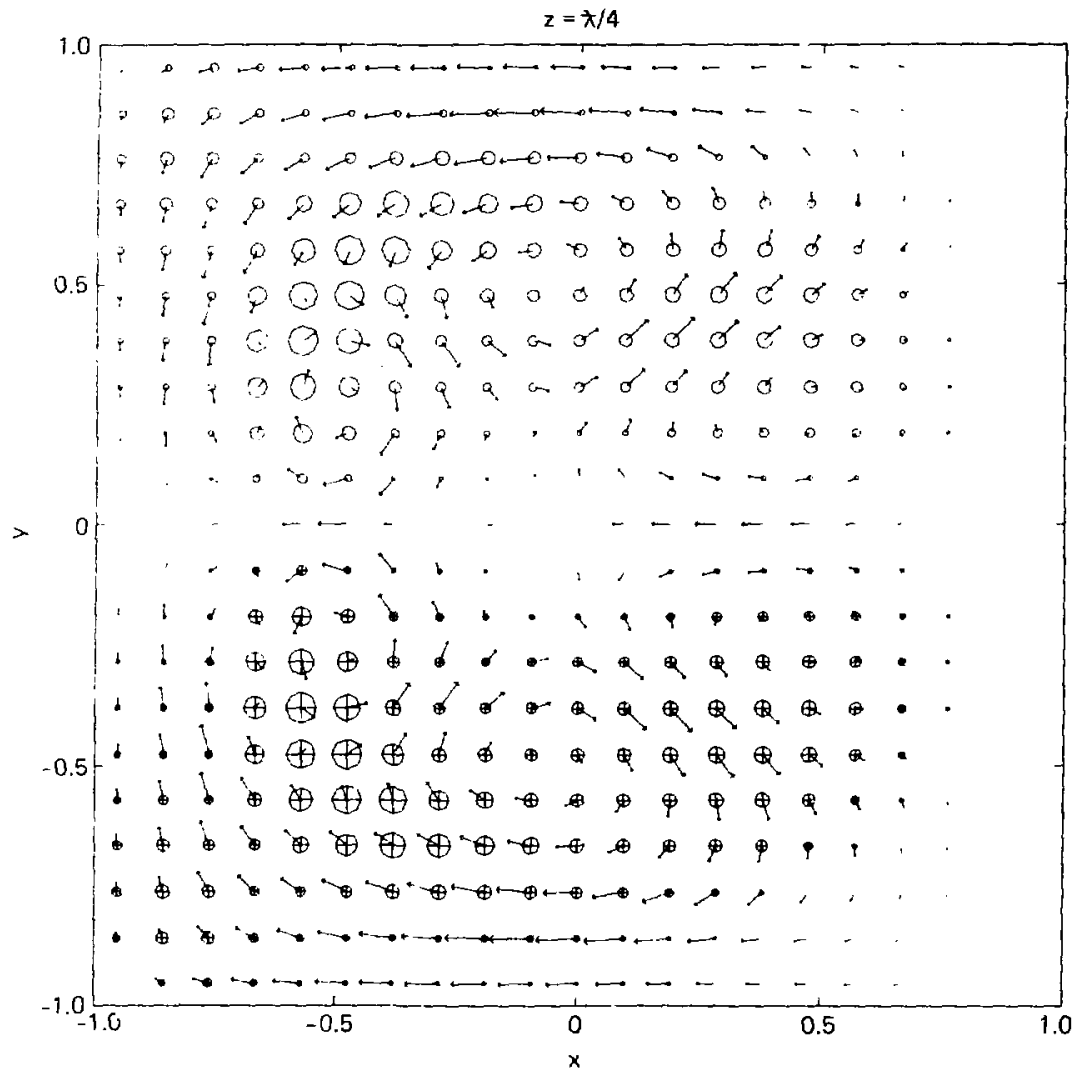

Maximum velocity in plane (arrows) $=4.79212243 \mathrm{E}-02$

Maximum perpendicular velocity (circles) $=5.42713987 \mathrm{E}-02$

Figure IV.15b The velocity field at $z=\lambda / 4$. 
Delta t
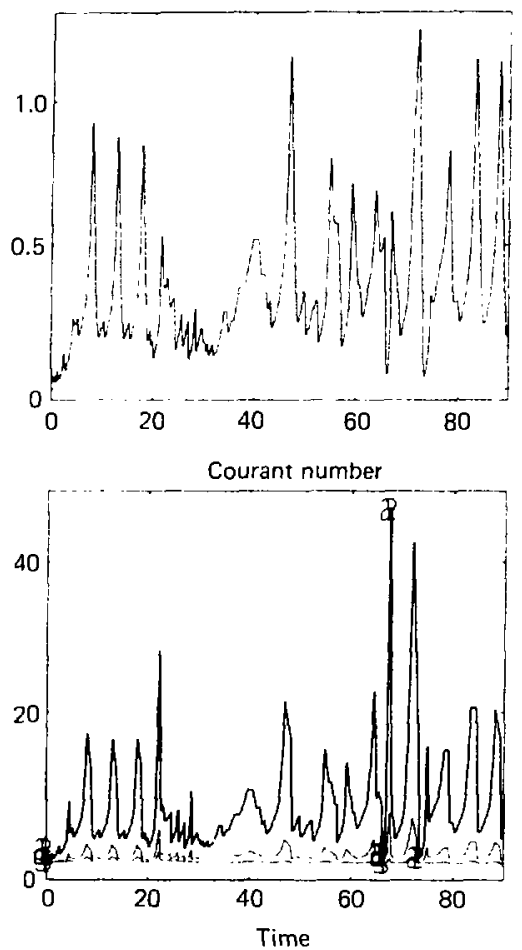

Delta t vs N

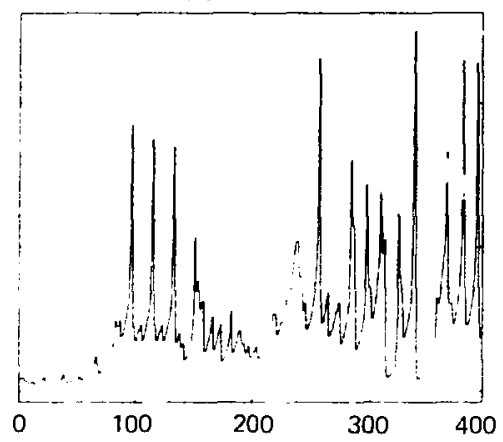

Courant number vs $\mathrm{N}$

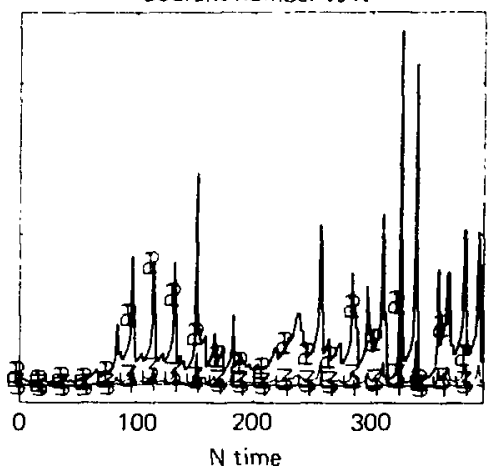

Figure IV.16 lhe time step size $j$ t and the Courant numbers used for the long time resistive simulation. 


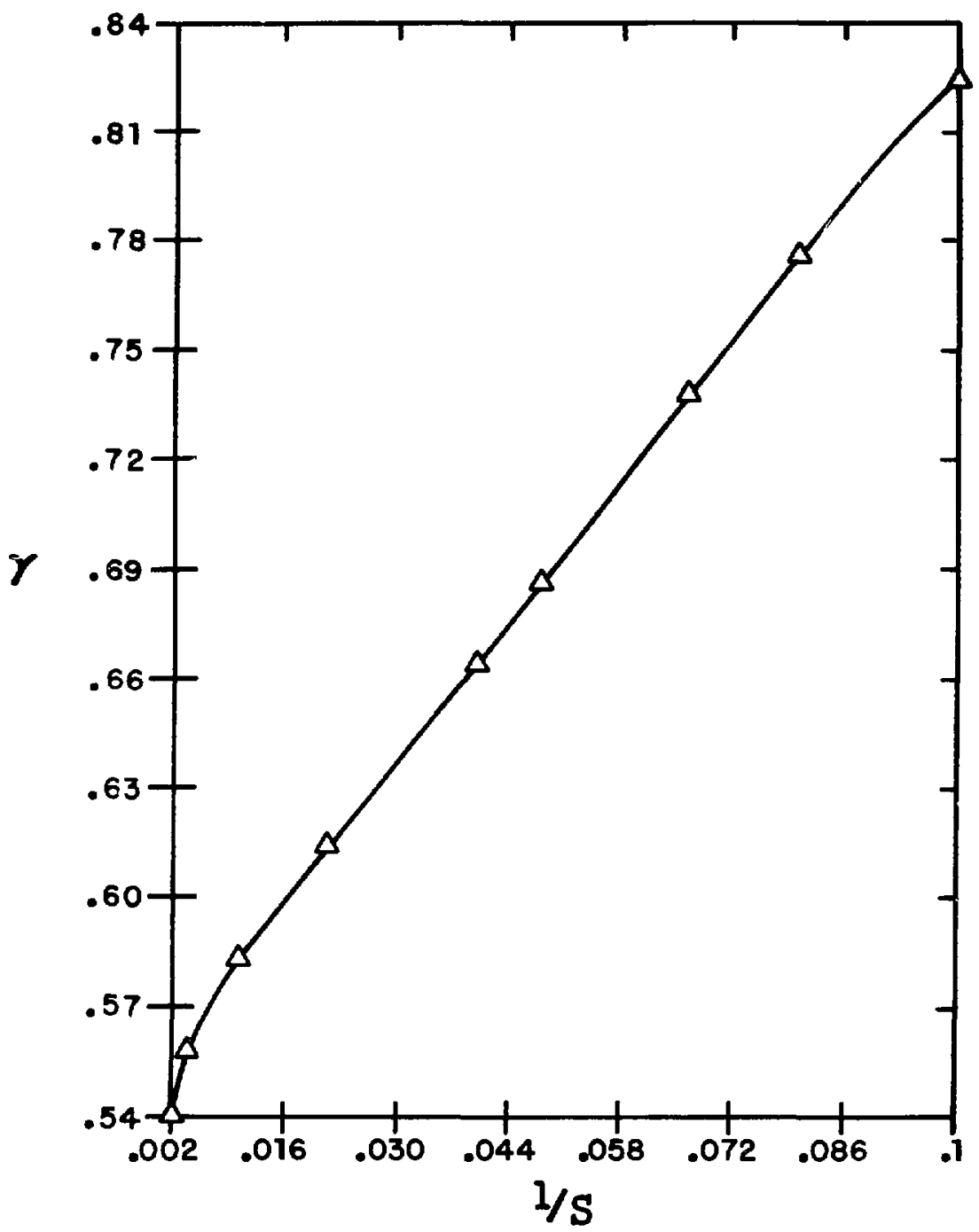

Figure IV.17 The growth rate plotted versus the inverse of the magnetic Reynolds number $S$. Note the increase in the growth rate with decreasing resistivity. 


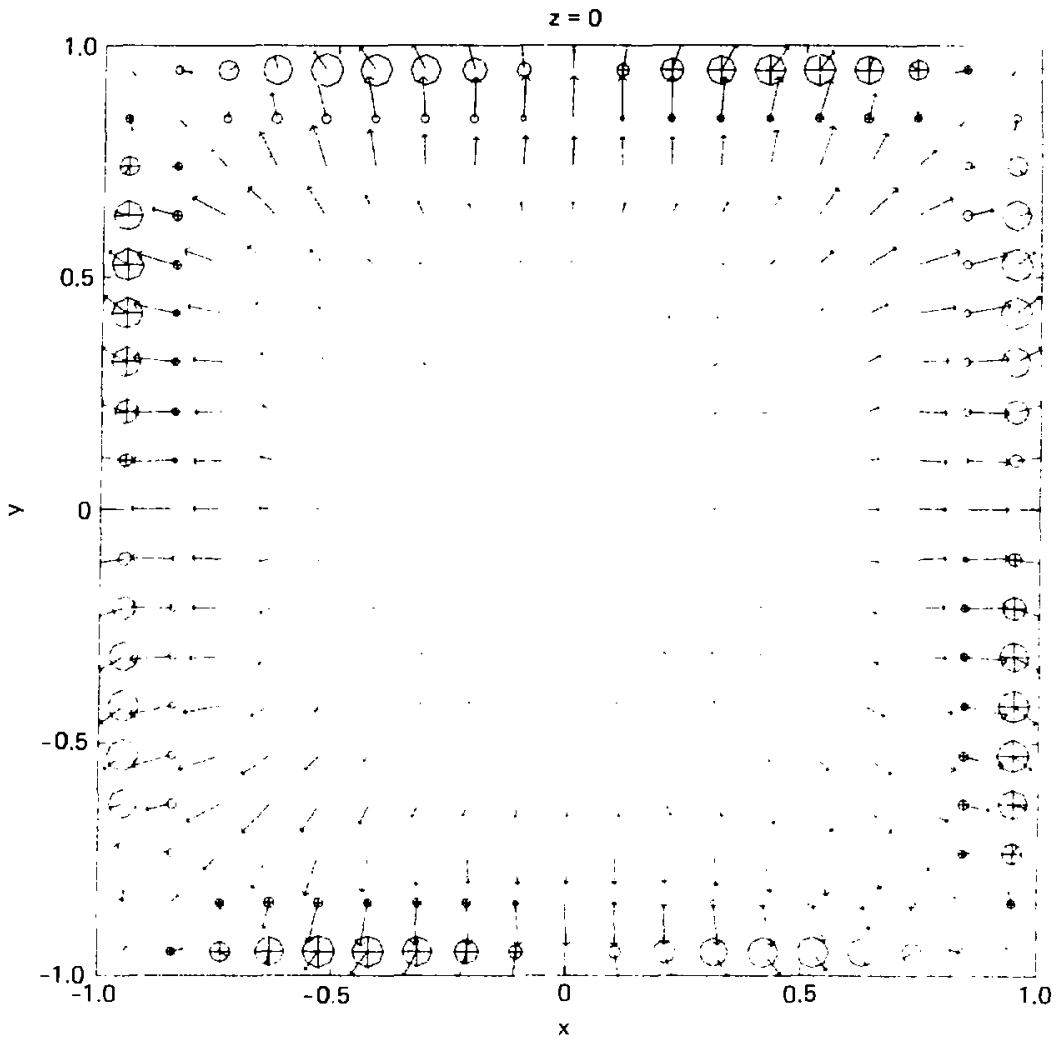

Maximum velocity in plane (arrows) $=7.09450652 \mathrm{E}-03$

Maximum perpendicular velocity (circies) $=4.05016837 \mathrm{E} \cdot 03$

Figure IV. 18 a 'l'he spontaneous velocity field caused by magnetic field diffusion. The initial perturoation has effectively disappeared from the velocity field. 


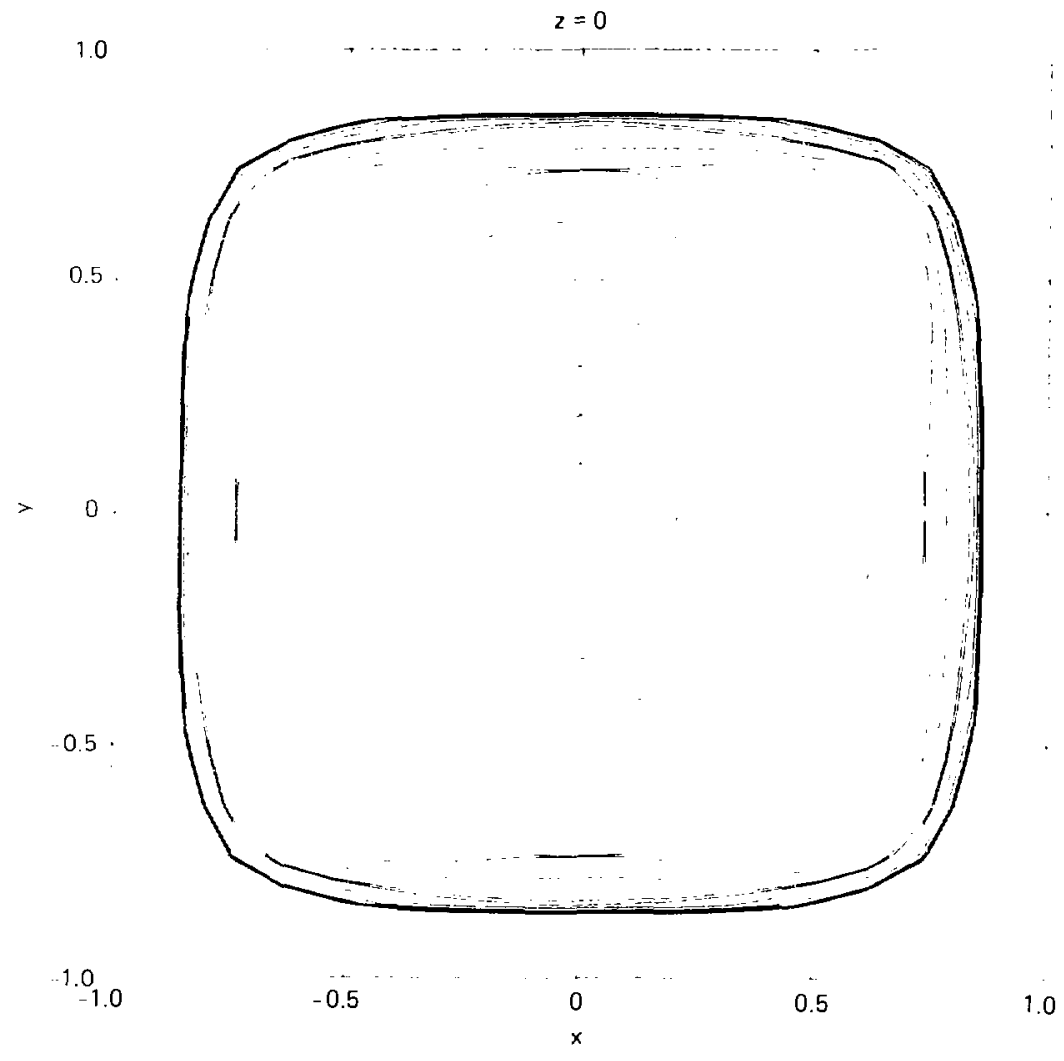

Density isoplot

Figure IV.IYh Contours of constant density at the $z=0$ plane are displayed. Note the steep gradients caused by the spontaneous velocity flows. 


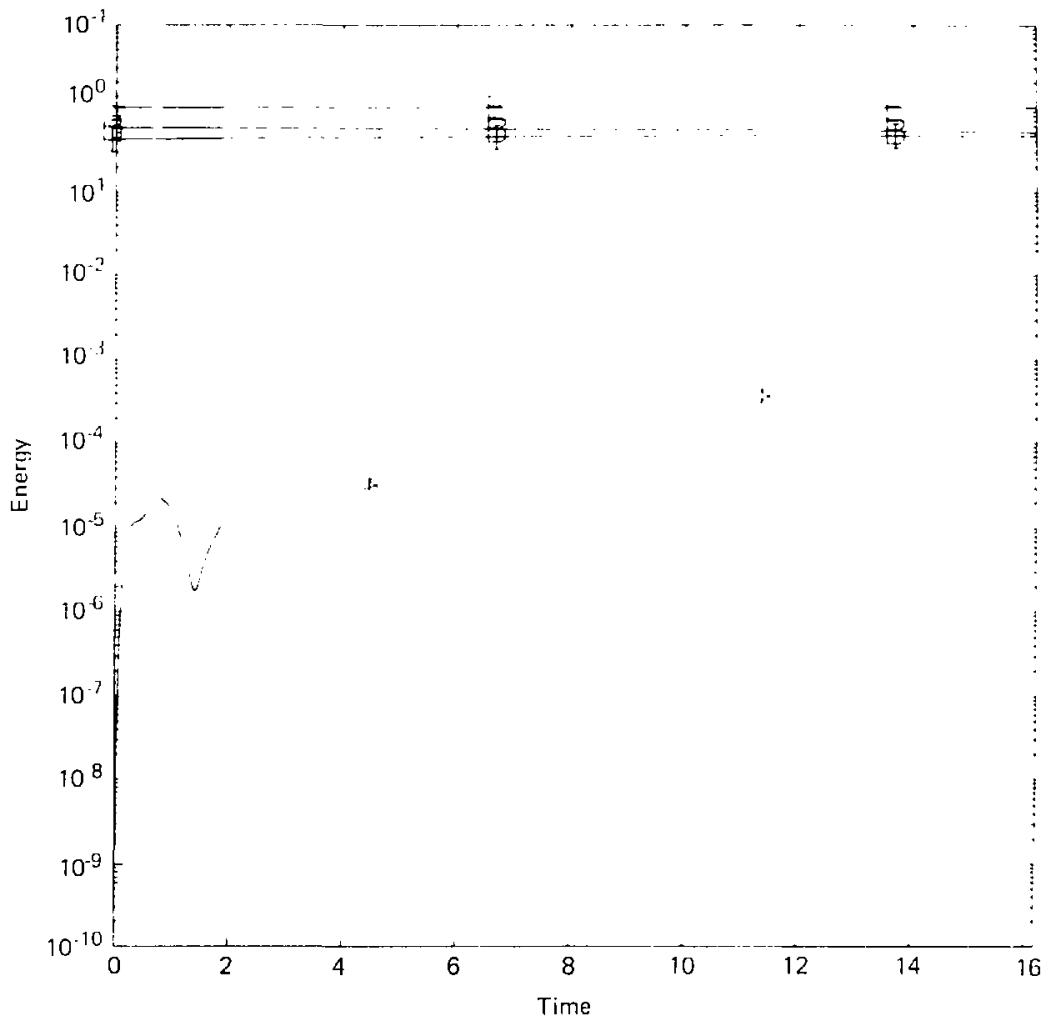

Figure IV.IYa the kinetic energy versus time for the resistive plasma with no balance terms. 


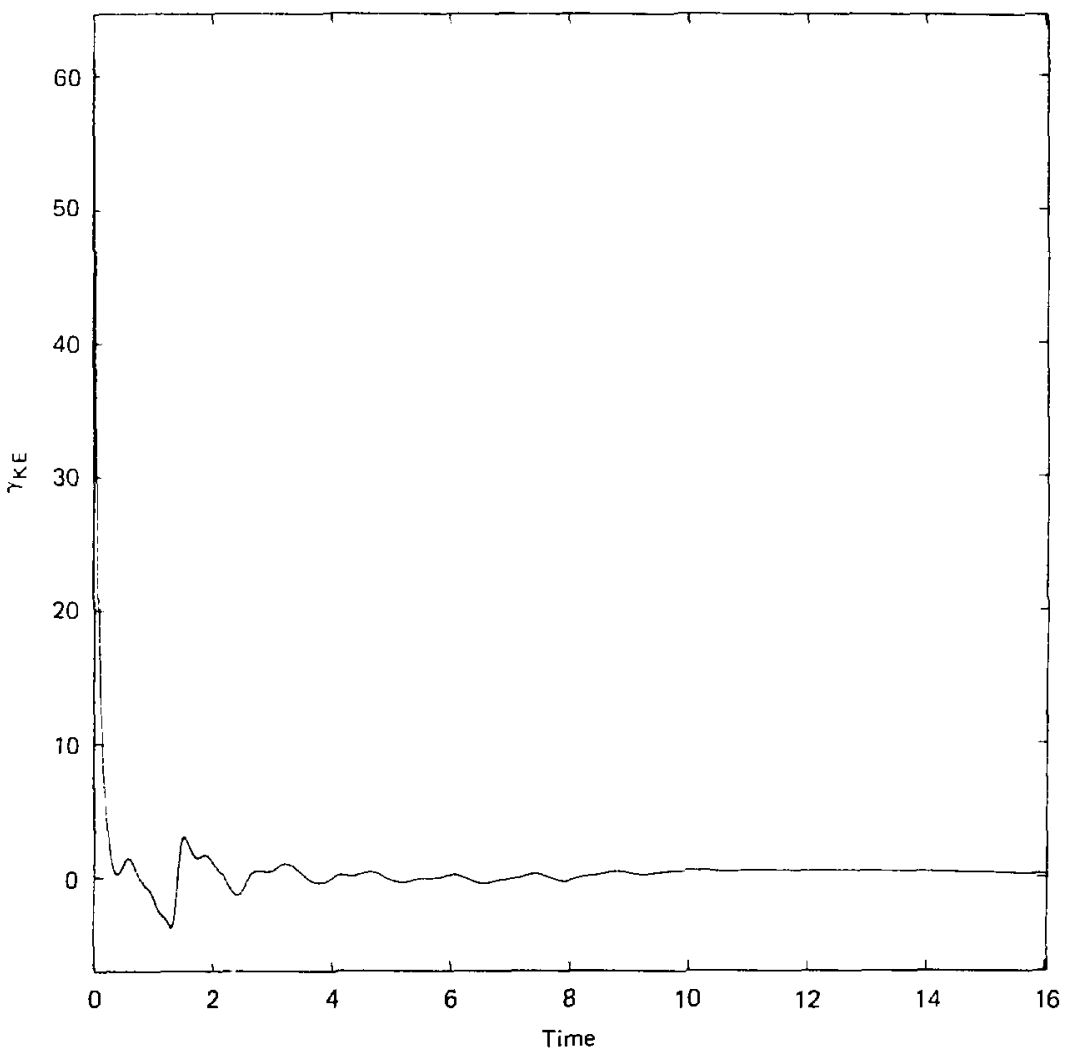

Figure IV.19b The growth rate versus time based on kinetic energy for the resistive plasma with no balance terms. The large initial growth hides the growth of the instability. 


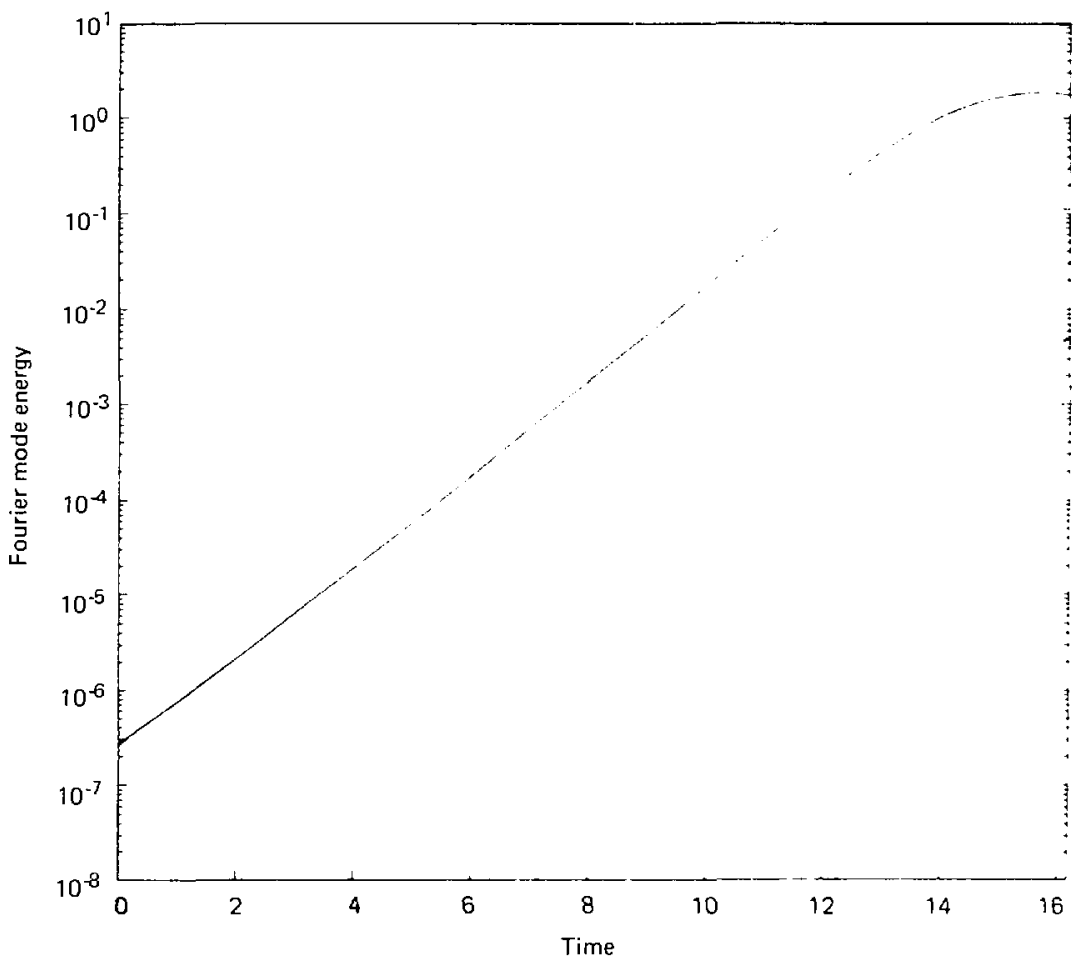

rigure IV. $19 \mathrm{c}$ The energy in the $\mathrm{n}=1$ fourier mode versule time or the same simulation. Note that the mode exhihits imediate exponential growth. 
$-140-$

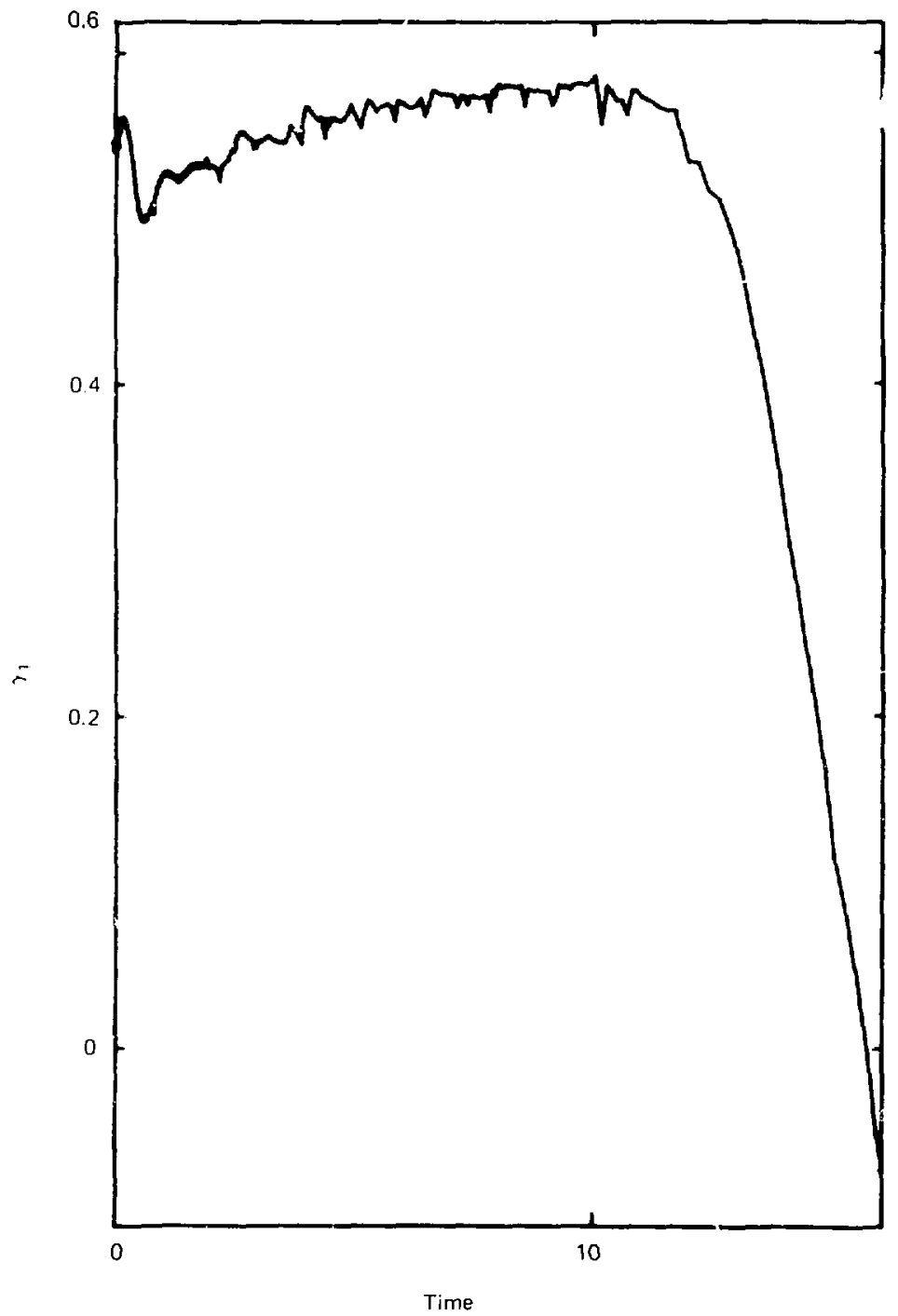

Figure IV.19d The growth rate of the $n=I$ Fourier mode plated versus time. The mode exhibits immediate exponential growth at a rate that is larger than that of the ideal plasma. 


$$
z=0
$$

1.0

$>\quad 0$.

i.

$0.5+t$

i.

$$
\text { + }
$$

$-t$

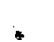

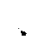

+

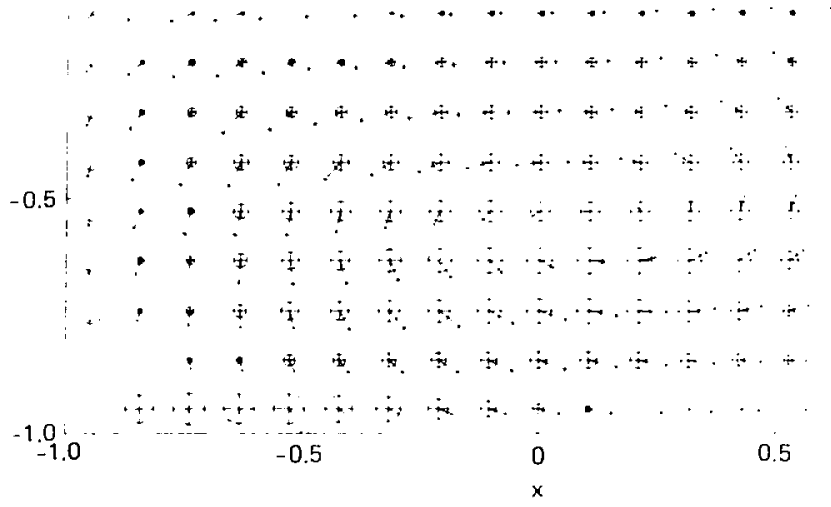

Maximum velocity in plane (arrows) $=1.75669538 \mathrm{E} .02$

Maximum pernendicular velocity (circles $=1.493866835-02$

Figur: IV.20a The $1 / 1$ mode can once again be seen in the relocity field at $t=9.45$. 


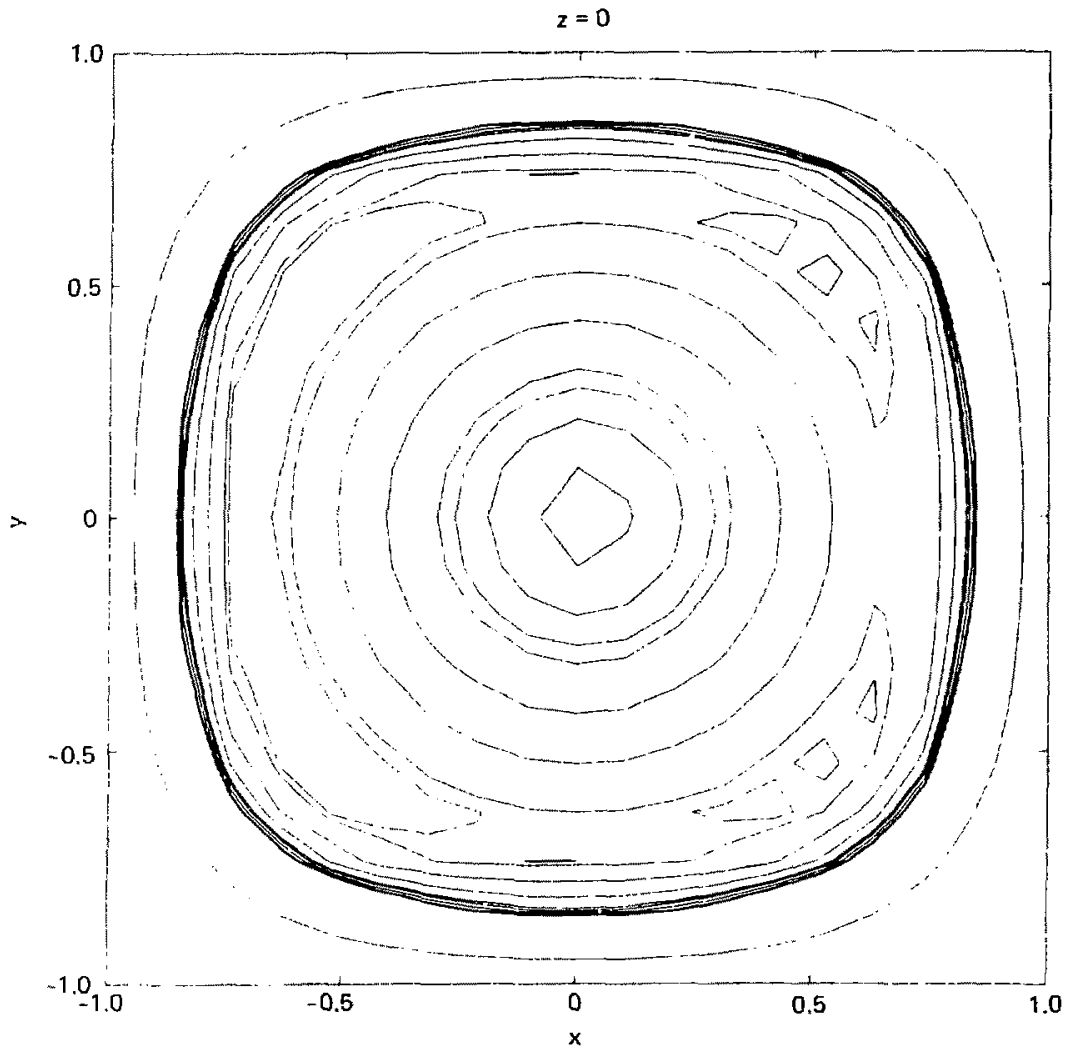

Density isoplot

Figure IV.20b The density isoplot displays a $m=1$ island that has emerged at $t=9.45$. 


$$
z=0
$$

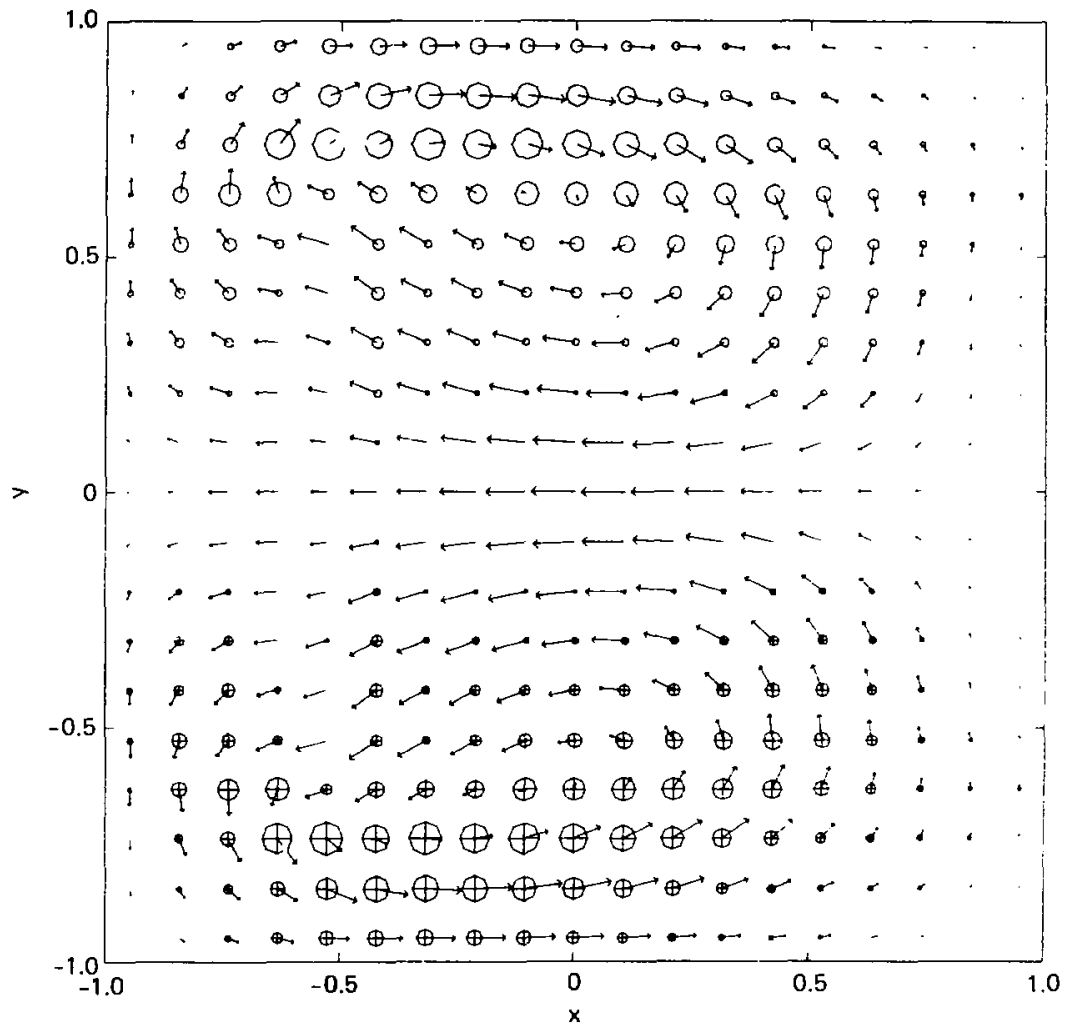

Maximum velocity in plane (arrows) $=3.95493446 \mathrm{E}-01$

Maximum perpendicular velocity (circles) $=4.58630870 \mathrm{E}-01$

Figure IV.20c The $1 / 1$ mode has saturated by $t=13.7$. The velocity field now shows a considerable nonlinear deformation. 


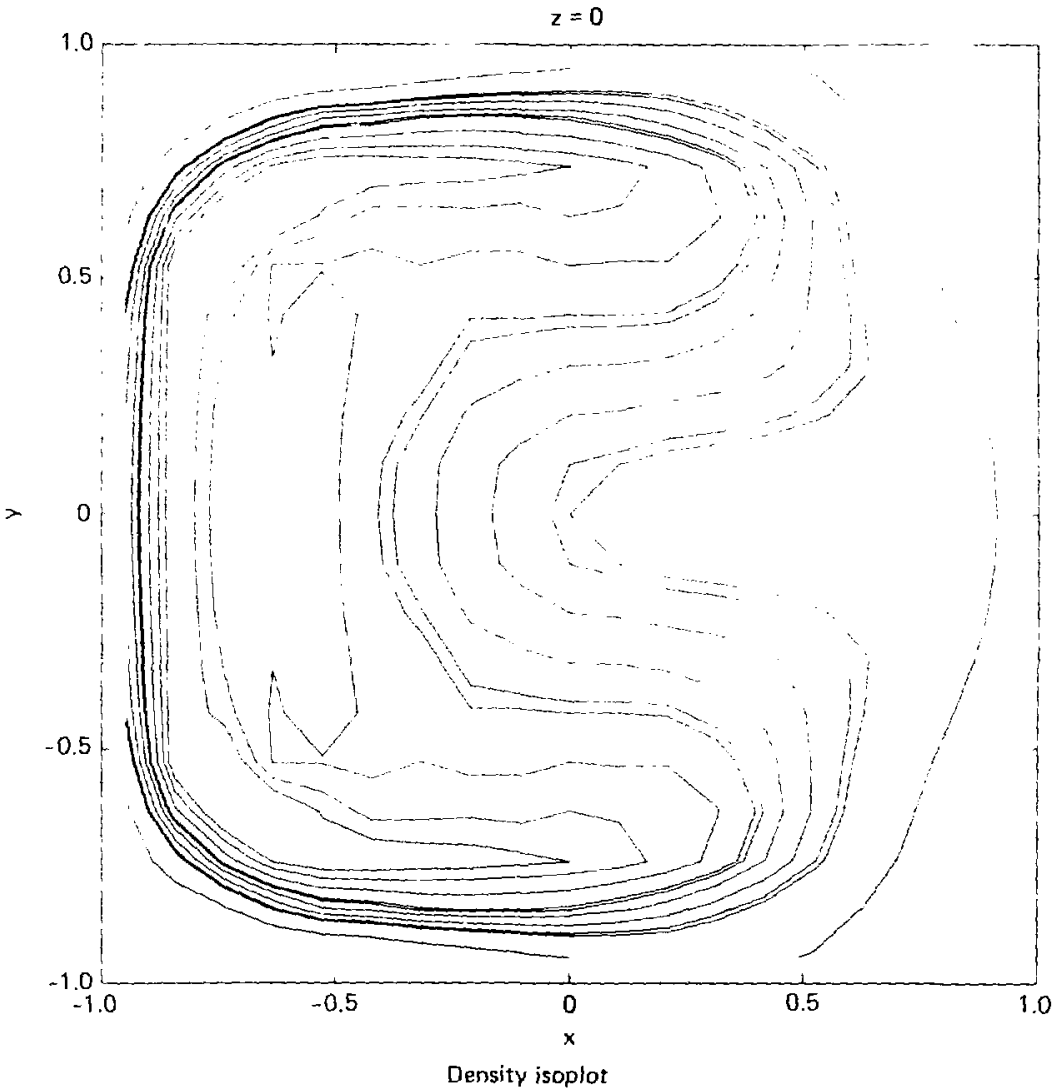

Figure IV.20d The $t=13.7$ density in which the plasma displays a considerable deformation towards the wall. 


\section{CHAPTER V}

THE INTERNAL KINK IN A SQUARE CROSS SECTION TORUS

\section{V.1 Introduction}

In the preceding chapter, the study of the intemal kink was 1 imited to the approximation of a large aspect ratio torus which was then treated in a straight Cartesian coordinate system. In this chapter, the effects of curvature are included by adopting the cylindrical coordinate system depicted in Fig. V.1.

This is done for three reasons. First, real toroidal devices do not have infinitely large aspect ratios. Typfcally, devices operate with aspect ratios from 3 to 5 . Therefore, we wish to see the error that has been max by the large aspect ratio assumption. Second, the Cartesian system of the last chapter does not verify the correct inclusion of the "Coriolus" terms in IMP's orthogonal curvilinear coordinate system. Comparison of IMP results with another toroidal code can be used for this verification. Finally, we wish to see if the curvature affects the final plasma state in a long time simulation.

The linear code of Bateman has been extended by Hicks and Wooten ${ }^{71}$ to include the effects of curvature on linear ideal MHD modes. This code is used to generate the linear eigenmodes used as perturbations in our code. The initial linear behavior of these modes in IMP is used to verify the correct inclusion of the "Coriolus" terms in our orthogonal curvilinear representation. 
The first case studied was an ideal $m=1, n=4$ internal mode in an aspect ratio ( $R / a) 4$ torus with the satety factor per unit wavelength on axis equal to .6 . This corresponds to the first case in the previous chapter. lhe growth rate determined in our model is within $1 \%$ of the predictions of the linear code.

An $m=1, n=2$ ideal internal mode is also simulated. This is a slowly growing mode and our growth rate differs from the linear value by $10 \%$

Finally, a simulation was pertormed which followed the long term development of the resistive plasma while allowing the ohmic heating to aftect the plasma dynamics. An $m=1, n=1$ mode was used to perturb a plasma in an aspect ratio 3 torus with a magnetic Reynolds number of $10^{4}$. The evolution of the perturbation was followed for 1000 Alfven transit times. The plasma, after initial motion caused by the resistive heating, exhibited growth of the $m=1, n=1$ mode. Ihis saturated and the plasma final $\perp y$ relaxed to a new axisymmetric equilibrium state that included a finite f $\downarrow$ ow field wnich displayed a pair of counter-rotating vortices at the outer radius (an $\mathrm{m}=1$ characteristic).

\section{2 Equilibrium and Initial Conditions}

The equilibrium used in this chapter is obtained numerically in the linear Hicks and Wooten code by the specification of an aspect ratio $A=R / a$, where $R$ is the major radius of the torus and a is its minor radius. The safety factor per unit wavelength $q_{c}$ on the maguetic axis of the torus of the 
equilibrium is then rescaled to give the desired $q_{c}$. This numeric equilibrium is not exact. Rather, the equilibrium has been iterated until the restdual error is below some required value. This error causes no problems in the linear code but induces a spontaneous flow in the nonlinear dynamic code. This flow masks the detection of low level perturbations by some of our diagnostics (e.g., the velocity fleld plots and the growth rates based on kinetic energy). In order to alleviate this problem, a set of fictitious forces are added to the momentum equation. These forces are calculated to exactly balance the errors in the equilibrium and, as such, do not vary in time. These forces allow us to observe the growth of the perturbation's velocity field and monitor its growth in the kinetic energy plots. Simulations have also been performed without the force balance terns which have shown that the instability growth rate, as monitored in the Fourier mode plots, is unchanged by these terms.

The equilibrium was once agala perturbed by using the eigenmodes of $B_{1}$, $\mathrm{v}_{1}$, and $\mathrm{P}_{1}$ that were generated by the Iinear code. Ti.es eigenmodes were calculated on the $z=0$ and $z=t / 4$ planes, combined via Eq. IV.3.3, and added to the equilibrium values.

\section{V.3 Boundary Conditions and Normalization}

The adiabatic, perfectly conducting wall boundary conditions of Section III.7.2 are used for the $r$ and $z$ coordinates since the wall is once again a flux surface of the plasma. The angular coordinate $\theta$ has periodic boundary conditions applied. 
The cylindrical coordinate system shown in Fig. V.1 corresponds to an ordering of $\underline{x}$ as

$$
\begin{aligned}
& x_{1}=z, \\
& x_{2}=r, \\
& x_{3}=\theta .
\end{aligned}
$$

The coordinate ranges are normalized to

$$
\begin{aligned}
& -1 \leq x_{1} \leq+1, \\
& A-1 \leq x_{2} \leq A+1, \\
& 0 \leq x_{3} \leq 1 .
\end{aligned}
$$

This gives us the set of scale factors

$$
\begin{aligned}
& h_{1}=b / a, \\
& h_{2}=1, \\
& h_{3}=2 \pi r / n,
\end{aligned}
$$

where $b / a$ is the squareness ratio of the torus and $n$ is the toroidal 4. ? number.

\section{V.4 Results}

\section{V.4.I Equilibrium Test}

The ideal MHD subset of our model was used to observe spontaneous flows in the unperturbed plasma caused by the lack of equilibrium 
force balance. These flows attained velocities of $10^{-2} \mathrm{~V}_{\mathrm{A}}$, two orders of magnitude above the normal input perturbation levels. Nex -, the force balance terms were activated and the code was rerun for 100 cycles. During this run, the spurious velocities generated were found to be of order of the machine roundoff ( $v^{\prime} s \approx 1 \times 10^{-16}$ ).

\section{V.4.2 $\underline{m=1, n=4}$ Ideal Kink Mode}

The $1 / 4$ ideal kink mode was run in an aspect ratic 4 torus whose safety factor per unit wavelength on the axis was chosen to be .6 . This corresponded to the case treated in Section IV.6.2. The growth rate obtained from the 1 inear code was $Y_{\text {LIN }}=.41,20 \% 1$ ess than that given in the straight geometry. However, this large growth rate still made the $1 / 4$ mode an attractive first candidate for simulation in the three-dimensional code. Figure V.2 displeys the initial equilibrium configuration of the plasma while Fig. V. 3 depicts the velocity field linear eigenfunction (generated by the 1 inear code) that was used as an initial perturbation. The constant $C^{\prime}$ of $\mathrm{Eq}$. (IV.3.3) was set to $10^{-4}$ and the mode was allowed to evolve. The plots of kjnetic energy versus time (Fig. V.4) and energy in the $n=4$ mode versus time (Fig. V.5) show that the mode began immediate exponential growth. The growth rate versus time based on the above energies (Fig. V.6) converged to a value $\gamma_{\text {IMP }}=.38$. This was within $7 \%$ of the 1 inear result. The simulation was continued until saturation was obtained at $t \tau^{2} 13$ transits. The velocities are of order $.29 \mathrm{v}_{\mathrm{a}}$ at this time.

Next, the simulation was run to the point where the growth rate had converged to .38 . At this point, the timestep size was cut to $10^{-2}$ to increase the accuracy of the simulation and the growth rate 
calculation. This resulted in a calculated growth rate $\gamma_{\text {IMP }}=.41$ which was within $1 \%$ of the linear code's result.

A resistive case was also run with a magnetic Reynolds number (S) of luo0. The growth rate and the final velocity attained at saturation was enhanced due to the effects of the resistivity, but the form of the eigenfunctions remained unchanged (see Fig. $\because .7$ ).

\section{V.4.5 $\mathrm{m}=1, \mathrm{n}=2$ Ldeal Kink Mode}

An $m=1, n=2$ internal mode in an aspect ratio 4 torus was also simulated in IMP. This longer wavelength mode has a much lower growth rate $\left(y_{\text {LIN }}=.08\right)$ as it is better wall stabilized than the $1 / 4$ mode. This mode required over 2000 timesteps for the linear code to converge on a $41 \times 41$ mesh. The resultant eigenmodes were then injected into the $19 \times 19 \times 24$ mesh used in IMH. The agreement between the linear code and our nonlinear one was not as good as in the $1 / 4$ mode ( $\gamma_{\text {IMP }}=$ .06); however, the simulation was performed at a large $\Delta t$ which did not give the best resolution of the growth rate. The kinetic energy and growth rate versus time and the velocity field at saturation $(t \approx 6 u)$ are given in Figs. $V .8$ and $V .9$. Note that the final

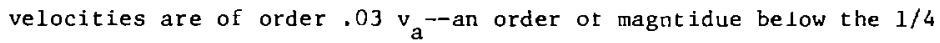
mode's final result. IMP required only 280 cycles to follow the plasma evolution tnrough over 60 AlEvén transit times. The Courant number for this run peaked at 5 and averaged 2.5. The maximum timestep used was $A t=.9$ 


\section{V.4.4 Long Time Resistive Simulation}

An $m=1, n=1$ ideal mode was started in an aspect ratio 3 torus with a magnetic Reynolds number of $10^{4}$. Magnetic diffusion of the equilibrium fields and ohmic heating of the plasma due to the equilibrium current was allowed to occur. The inftial velocity field perturbation (Fig. V.10) immediately had its form eroded by the effects of the finite resistivity of the equilibrium fields (Fig. V.11). There was an immediate jump in kinetic energy whlch was then followed by the growth of the $m=1, n=1$ mode. This growth was eastly visible on the Fourier mode plots (Fig. V.12) but was undistinguishable in the velocity and kinetic energy diagnostics (Figs. V.11, V.13). The growth rate calculated by IMP was $\gamma_{\text {IMP }}=.06$ while the linear, ideal growth rate was $Y_{L I N}=.015$. This large discrepancy can be attributed to the timestep size not having been 1 imited to be a small fraction of an Alfuen transit time during the period of linear growth ( $\Delta \mathrm{t} \imath 1-?$ Alfuen transit times). These timesteps corresponded to Courant numbers of 10-20. During the latter parts of the simulation, the maximu timestep of 5 Alfven transit times was attained (Fig. V.14). This corresponded to a Courant number of 60 . The average Courant number for this probiem was 40 . At $t=58$, the 1,1 mode again became visible in the velocity field plots (Fig. V.15), while saturation of the mode began at approximately $t=70$.

As the simulation progressed, the interaction of the higher $n$ modes caused the plasma to relax to a final ixisymmetric equilibrium that possessed an $m=1, n=0$ flow fleld (Figs. V.16, V.17). 


\section{V.5 Summary}

Our model has been applied to ideal and resistive plasmas in a toroidal geometry. Good agreement has been displayed between the growth rates predicted by the linear code and those calculated with IMP. In addition, a long term simulation which displayed the relaxation of a resistive plasma to an axisymmetric equilibrium with a finite flow field was presented.

Once again, the ability of our model to run at courant numbers substantially greater than unity was displayed. 


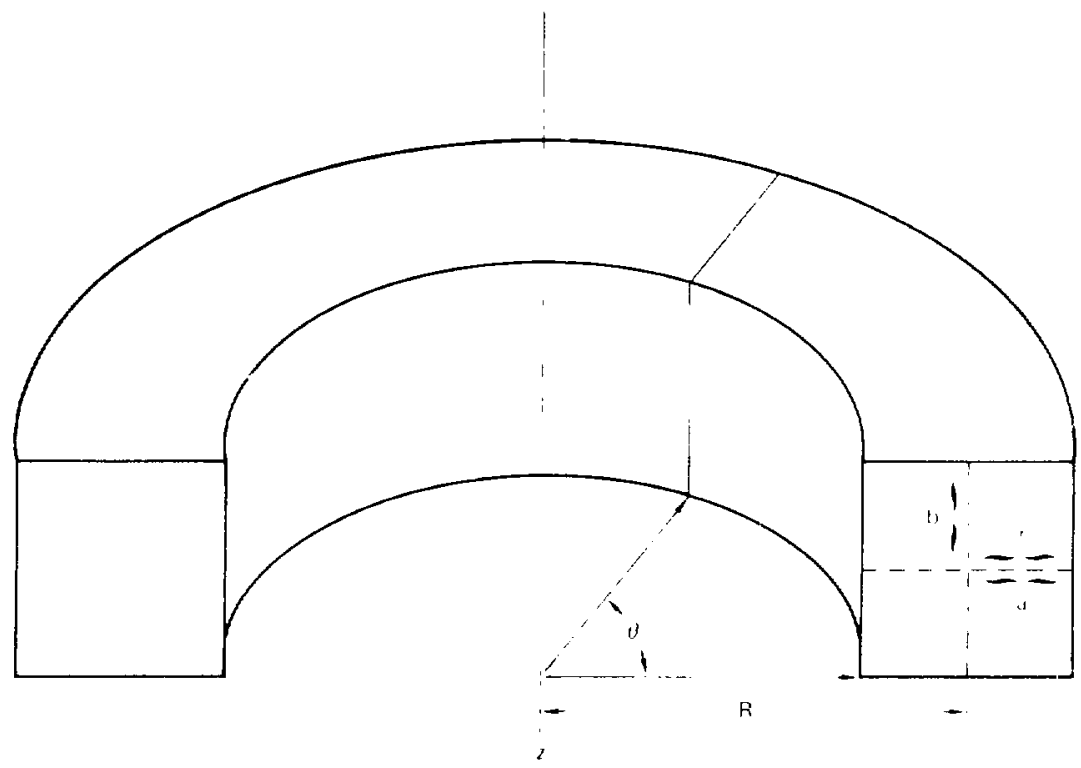

Figure 1

lhe cylindrical coordinate sytem used in this chater. The major radius is $R$ and the minor ralitu is at ir $r$. The squareness ratio is sriven by b/a. Nl lengtha are seasured in units of the minor radius it. 
$\theta=0$

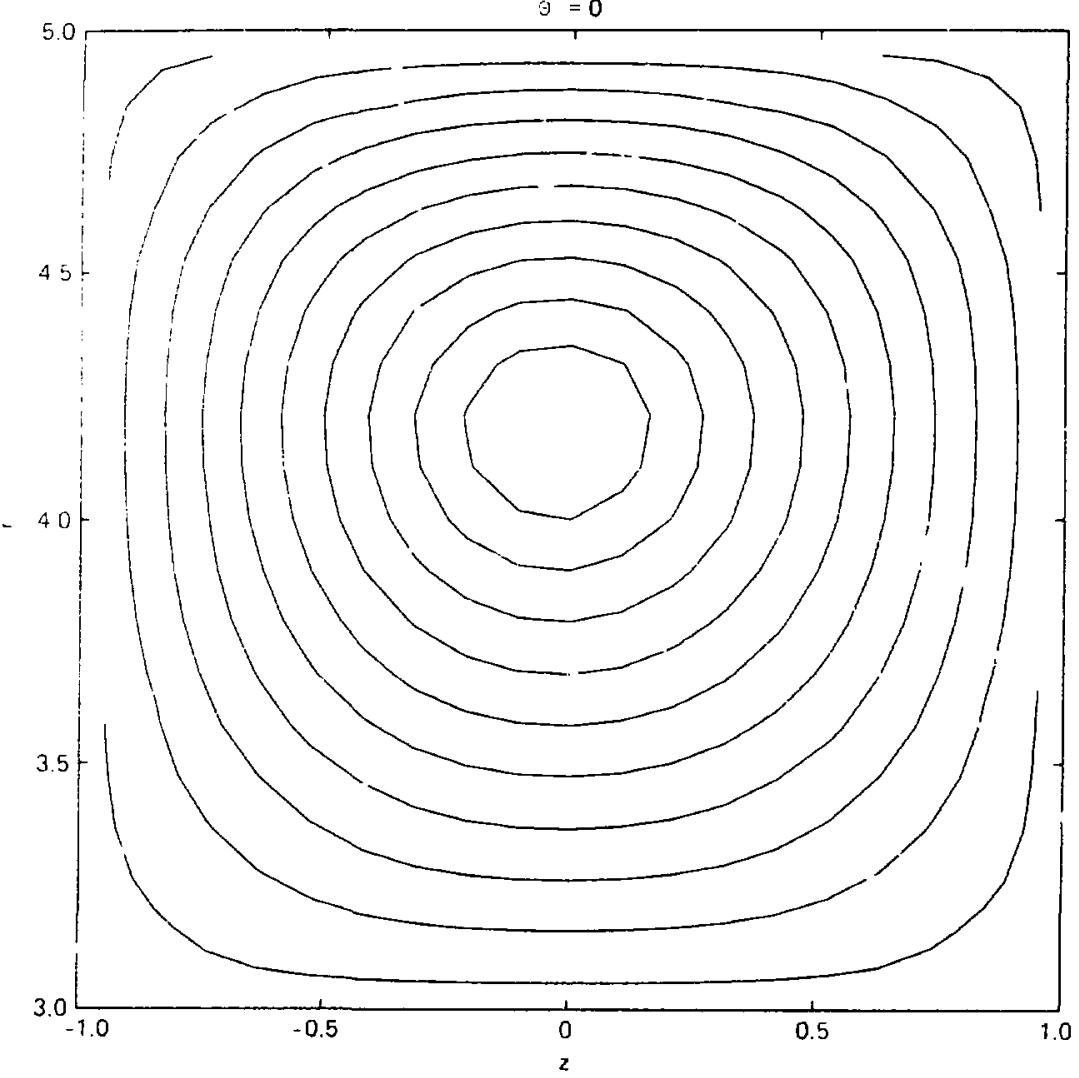

Pressure isoplot

Figure V.2

The equilibrium pressure contours for an asnect rario 4 torus are shown as found numerically by the equilibrium solver in the linear cole. Note that the pressure peak is uisplaced toward the cuter wall. Contours of constant magnetic flux are identical in form to these pressure contours. 


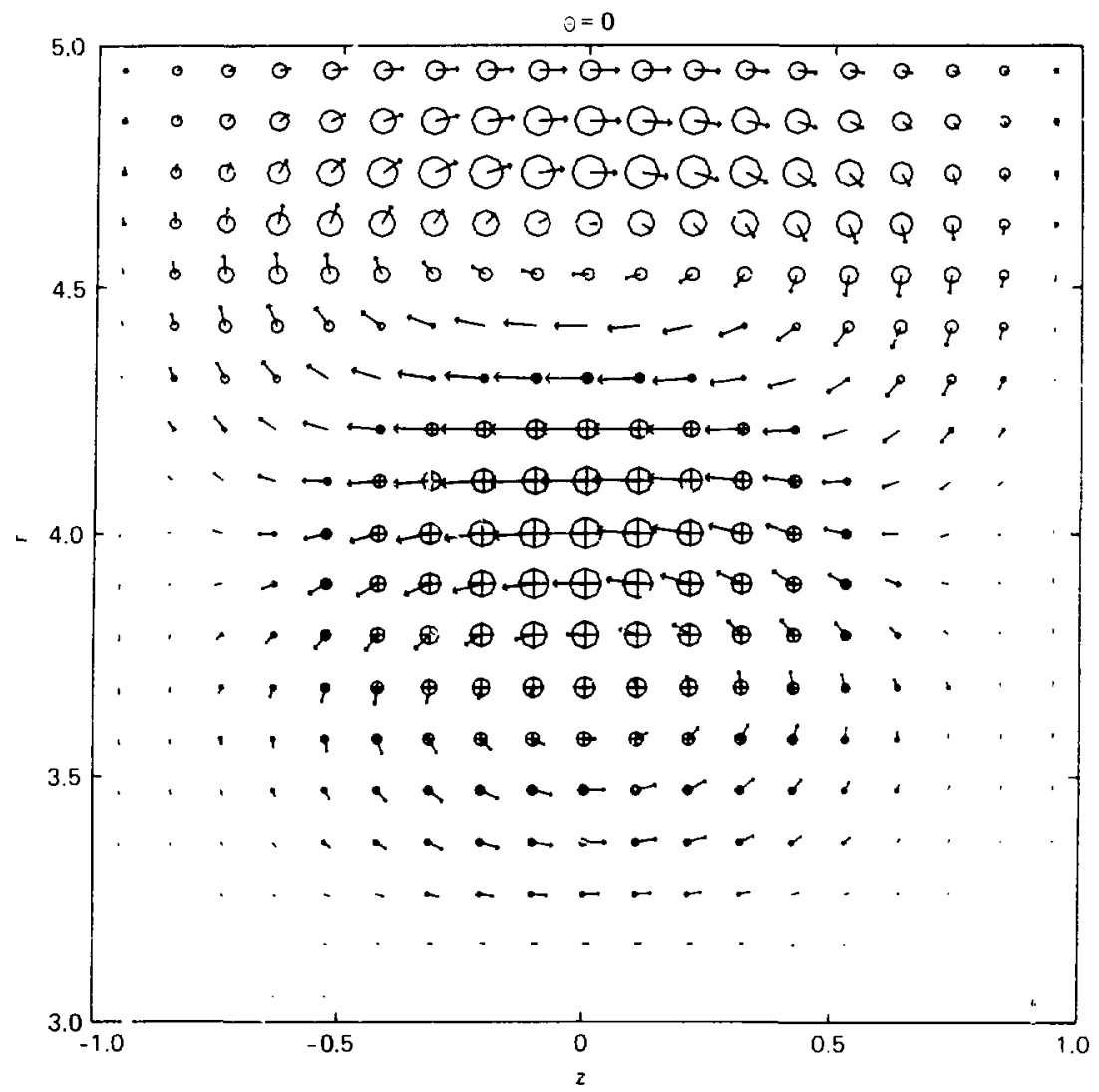

Maximu, $n$ velocity in plane (arrows) $=1.41774478 \mathrm{E}-02$

Maximum per pendicular velocity (circles) $=9.68574569 \mathrm{E}-03$

Ficire r.ja

The velocity field generated by the linear code and projected on the $:=0$ plane. 
$\theta=\pi / 4$

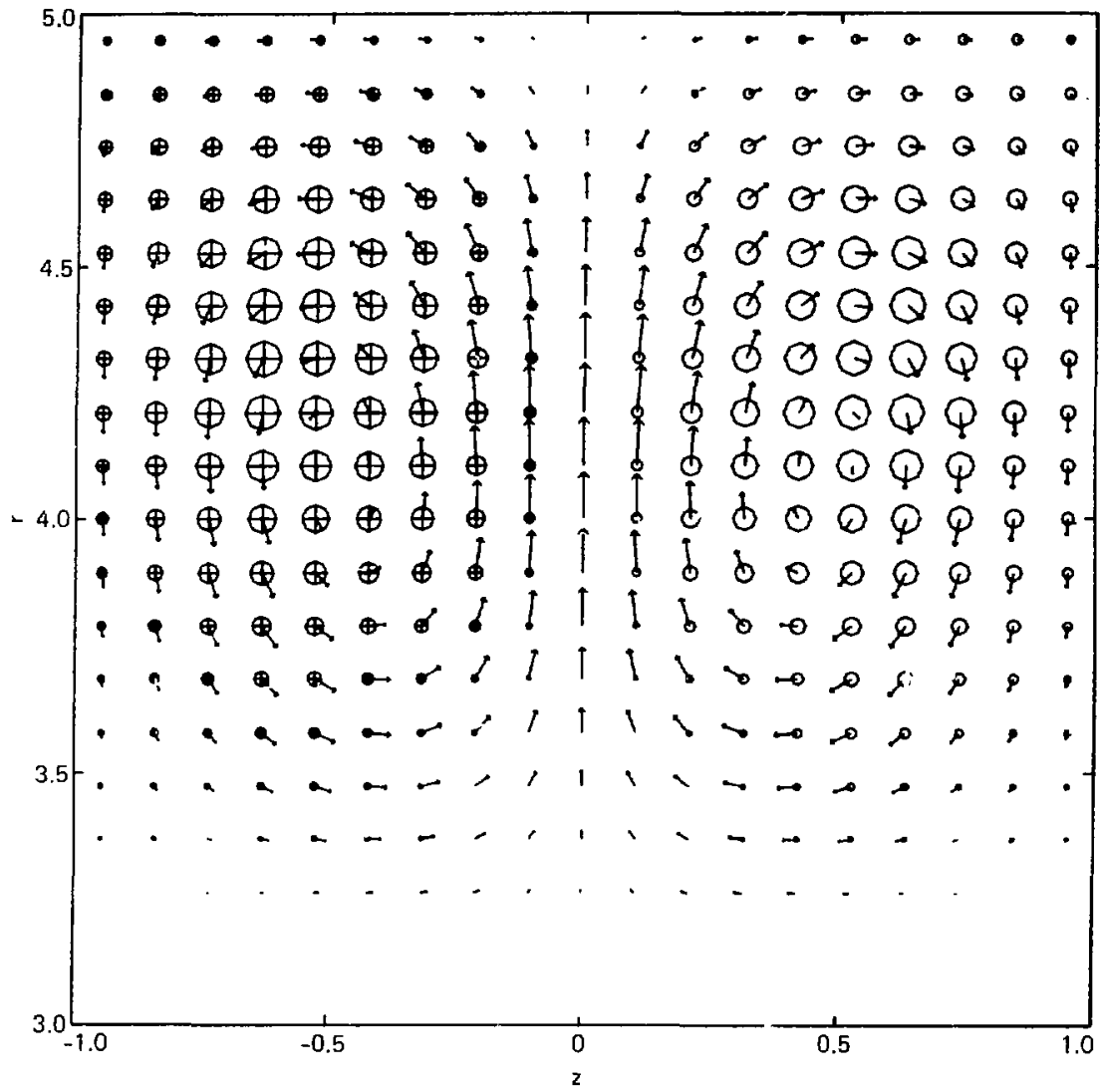

Maximum veiocity in plane (arrows) $=1.29026849 \mathrm{E}-02$

Maximum perpendicular velocity (circles) $=7.36848347 \mathrm{E}-03$

Figure V.3b

The velocity field generated by the linear code and projected on the $\Theta=t / 4$ plane. 


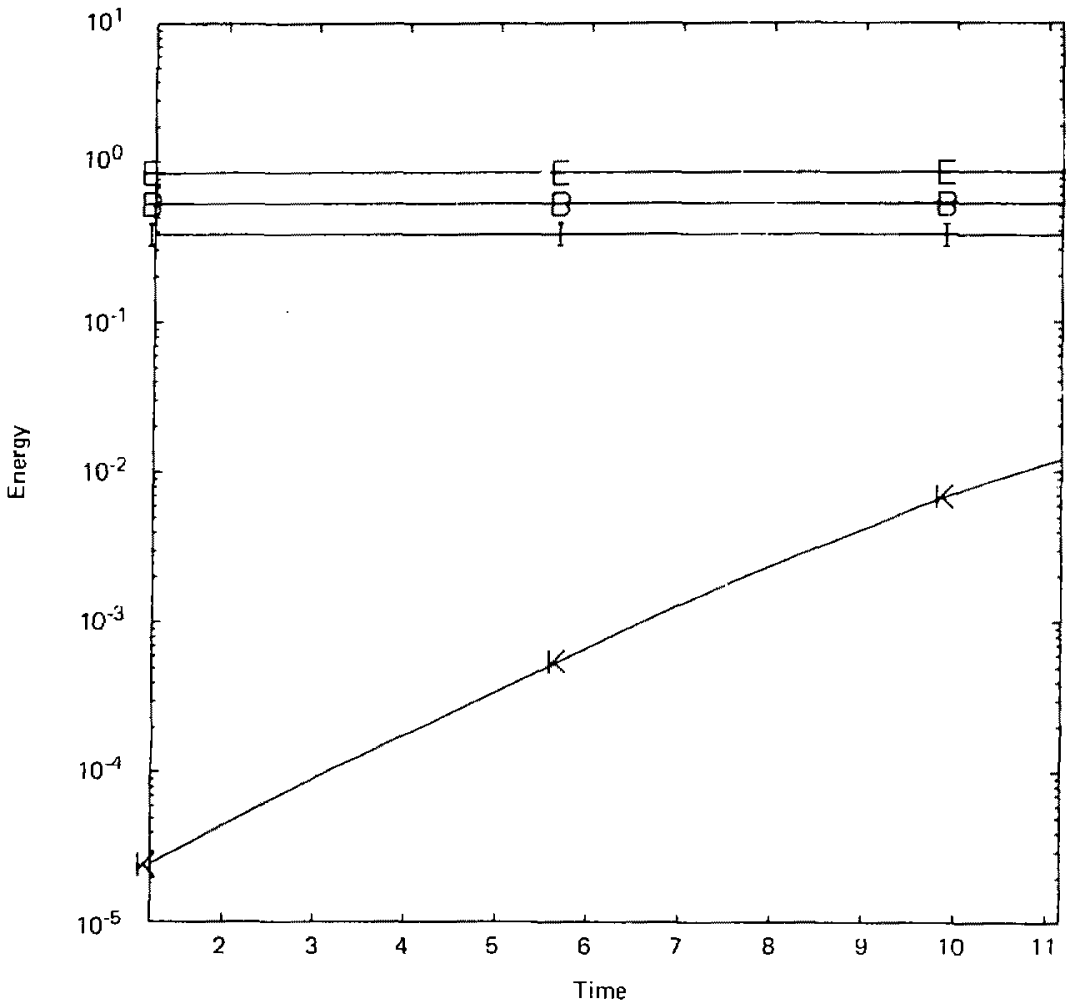

Figure V.4

The energy is plotted versus time for the $1 / 4$ ideal kink mode simulation. 


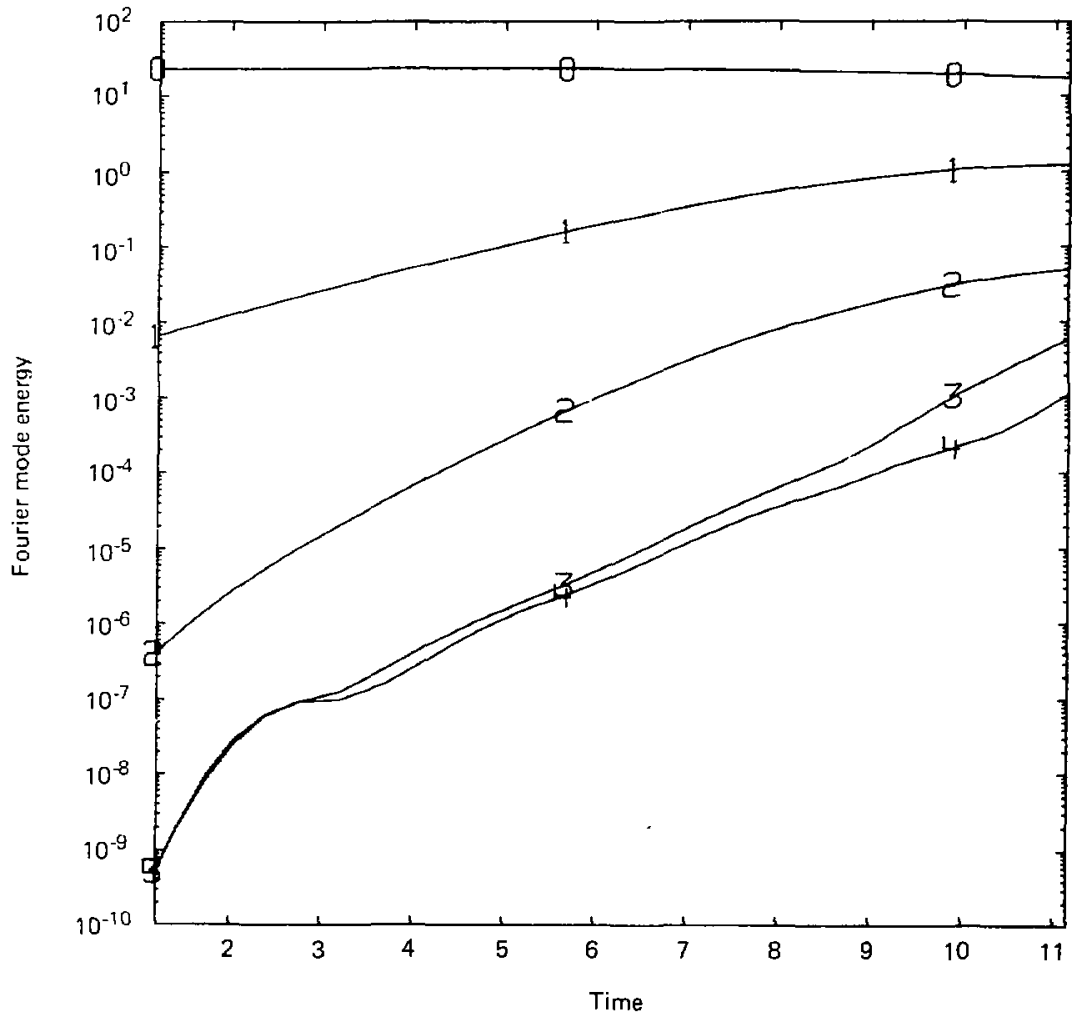

Figure V.5 The energy in the $\mathrm{n}=4$ Fourier mode versus time is given for the $1 / 4$ ideal kink simulation. The mode displays an immediate exponential growth. 


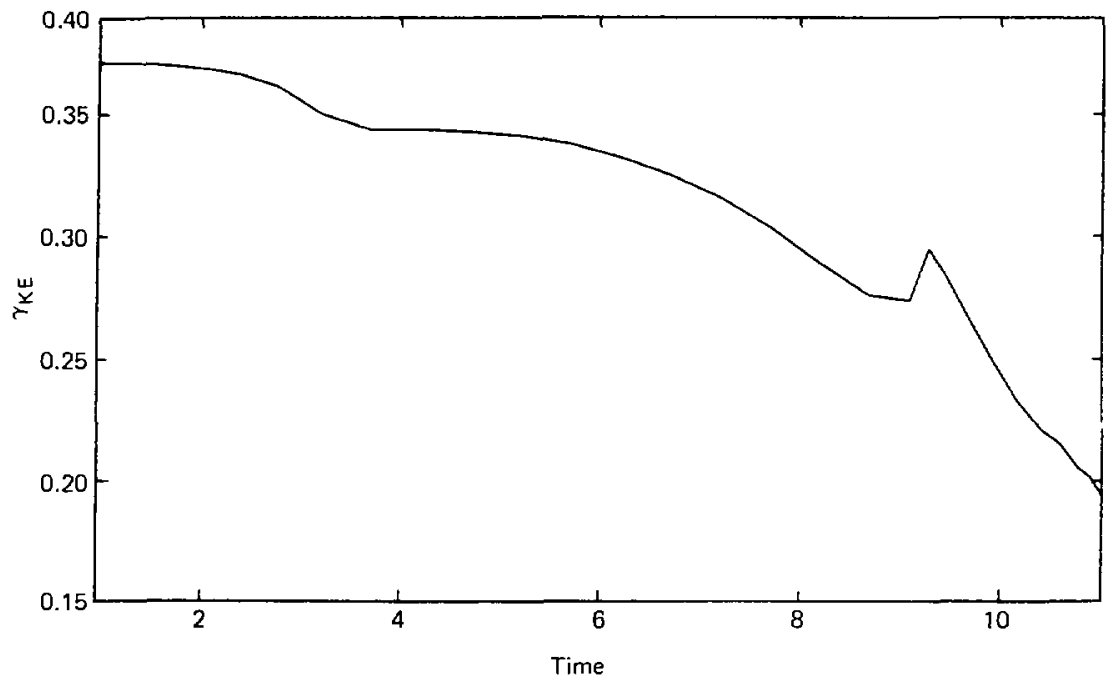

Figure V.6a The growth ate based on the kinetic energy (Figure V.4). 


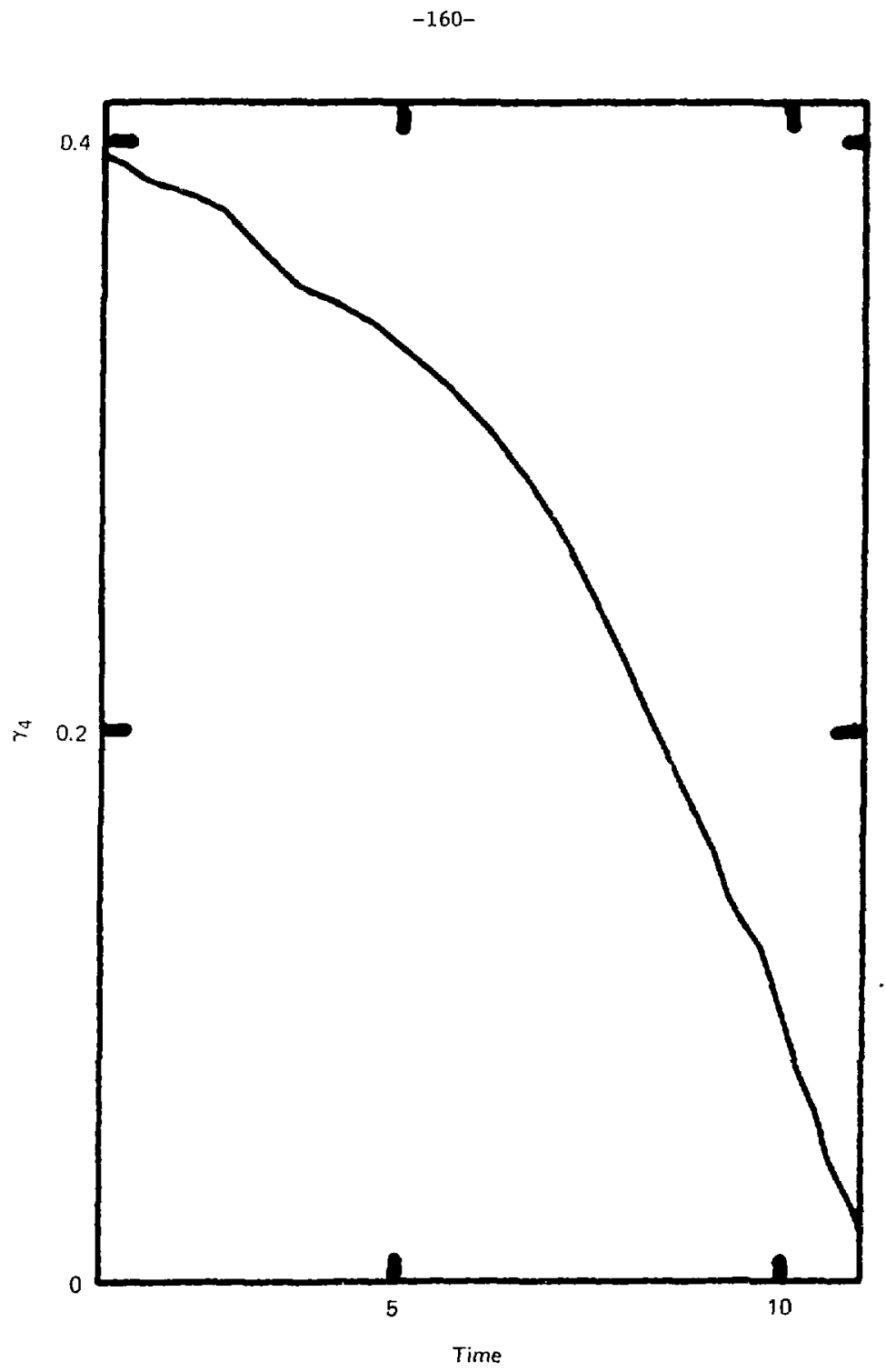

Figure $Y .6 \mathrm{~b}$

The growth rate based on the energy in the $n=4$ Fourier mode (Figure V.5). 


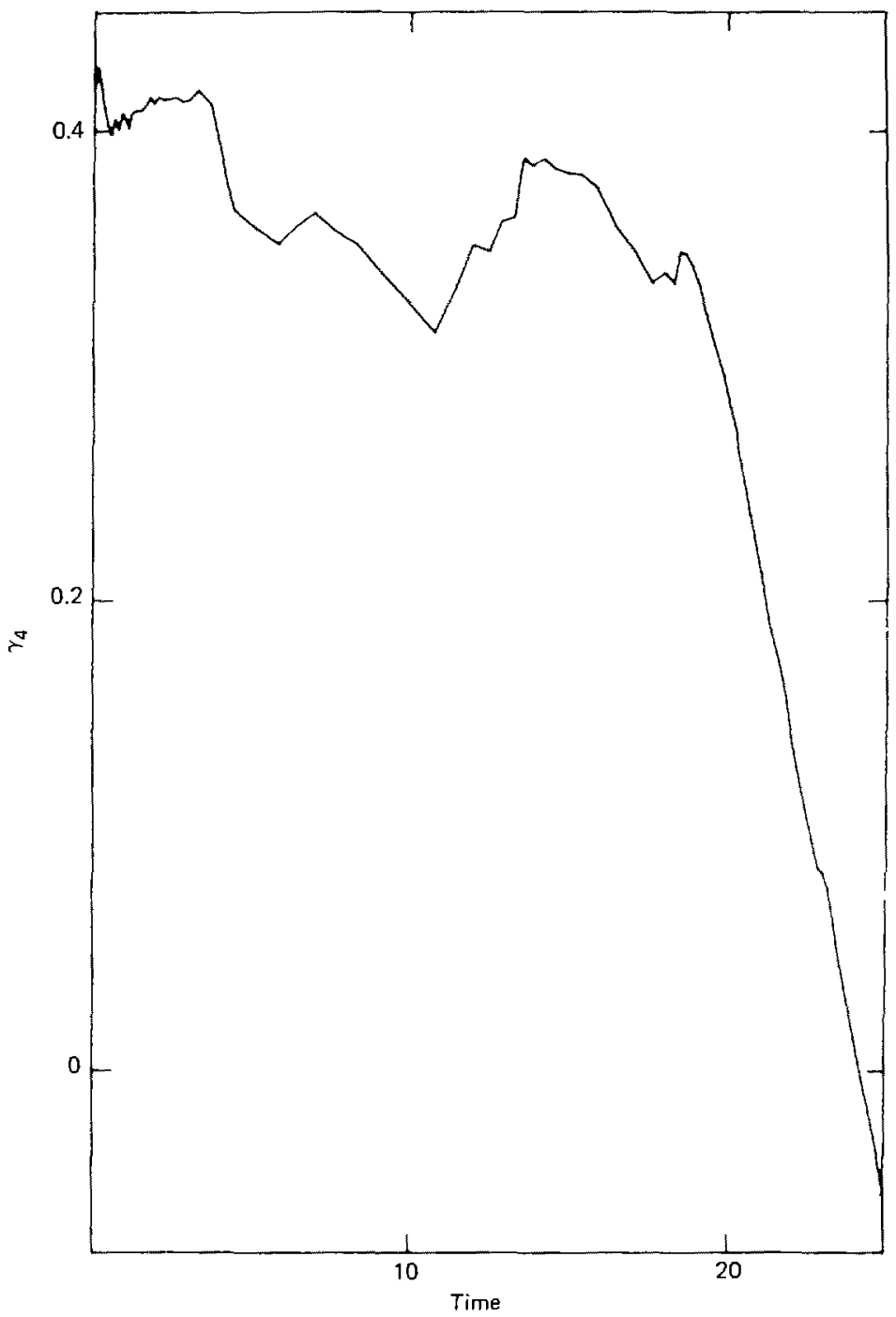

Figure V.7a The growth rate versus time for the $1 / 4$ mode in a $s=1000$ plasma based on the energy in the $n=4$ fourier mode. 


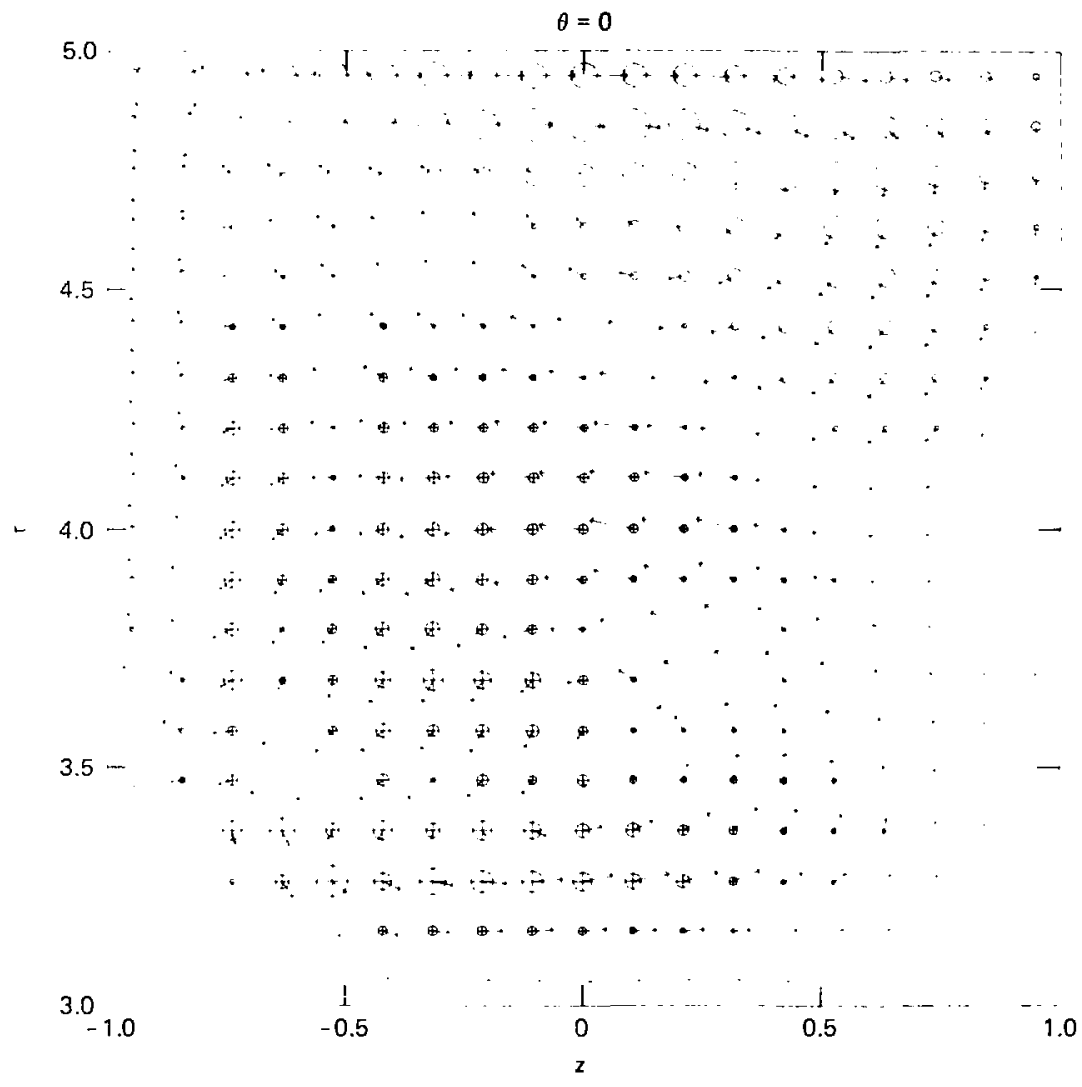

Maximum velocity in plane (arrows) $=3.50269972 \mathrm{E}-01$

Maximum perpendicular velocity (circles) $=4.57162242 \mathrm{E} \cdot 01$

Figure V.7b The velocity field on the $\theta=0$ plane at $t=25$ for the $1 / 4$ mode in a $s=1000$ plasma. 


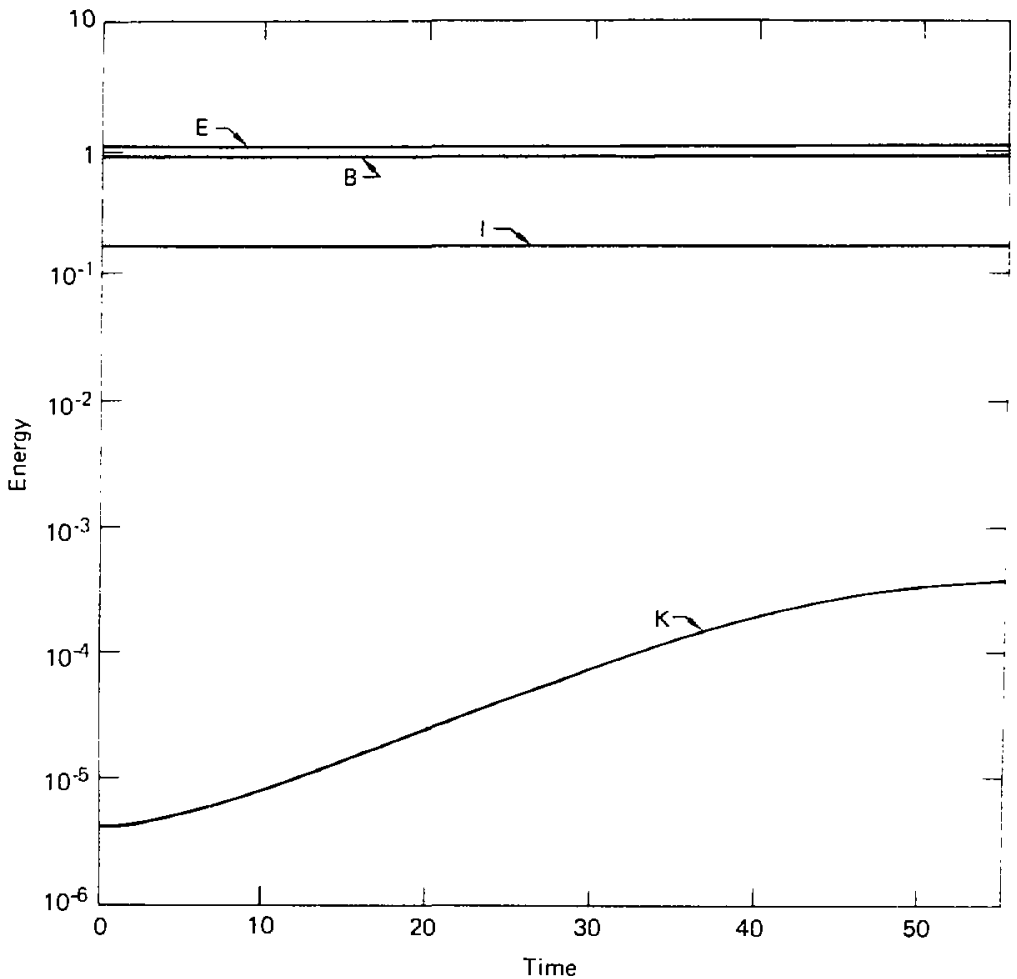

Ficure V.in The mergy nlntted versus time for the $1 / 2$ ideal kink sirulation. 


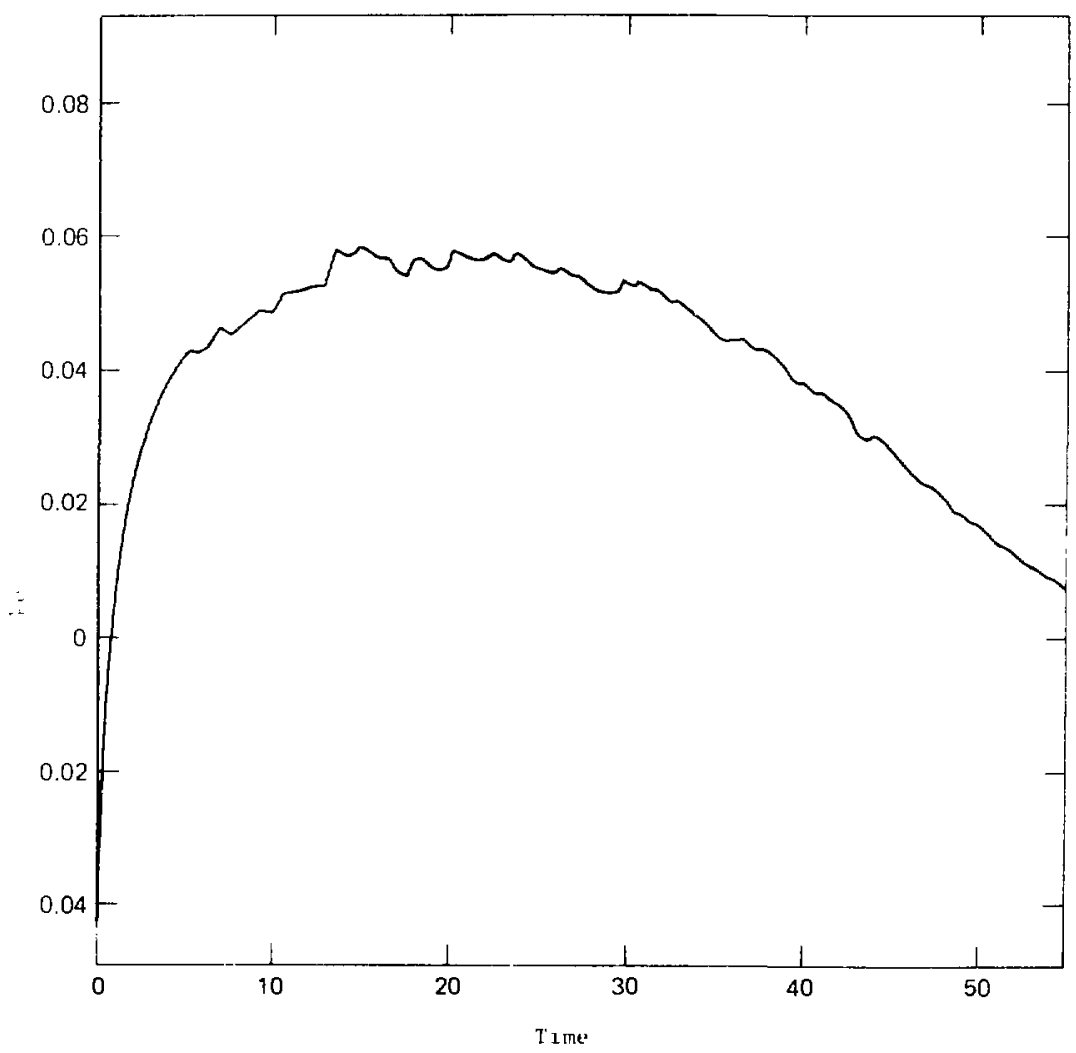

Figure $V, \underline{b}$ the growth rate of the kinetic energy plotted versus time for the $1 / 2$ ideal kink simulation. 
$n=0$

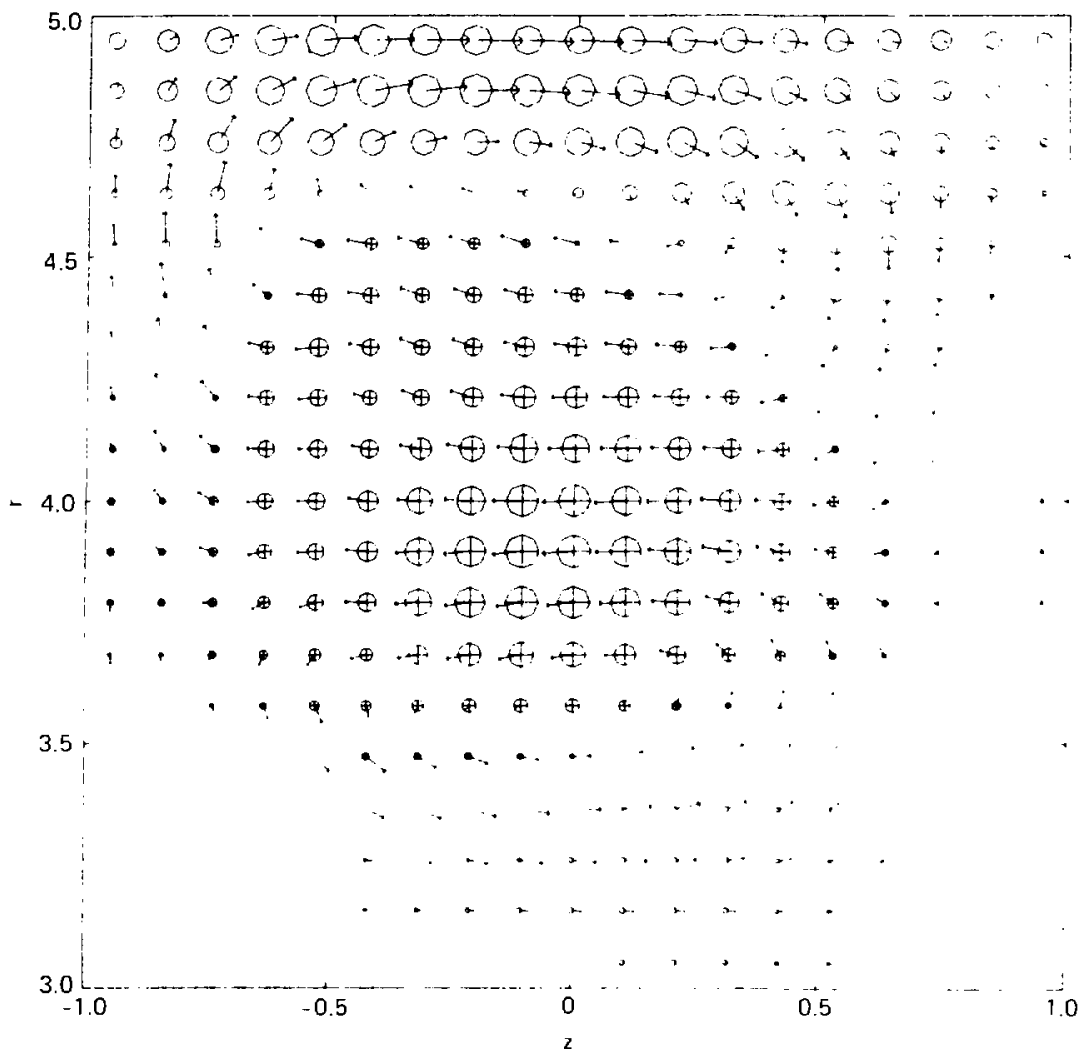

Maximum velocity in plane (arrows) $=2.96016052 \mathrm{E} \cdot 02$

Maximum perpendicular velocity (circles) $=2.52006645 \mathrm{E} .02$

Figure V.9a The velocity field on the : $=0$ plane at saturation are shown for the $1 / 2$ ideal kink simulation at $t=72$ Alfuén transit times. 


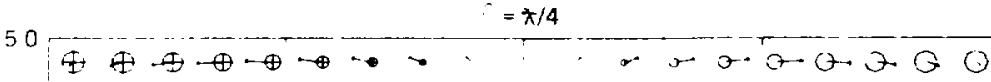

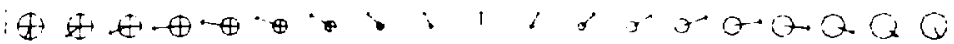
I

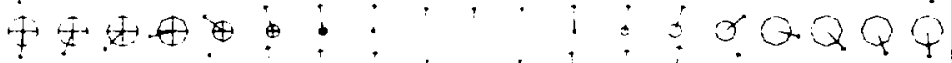

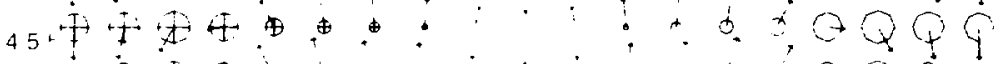

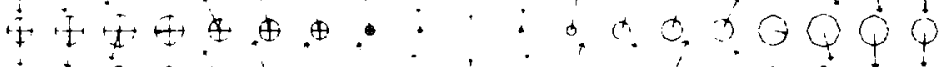

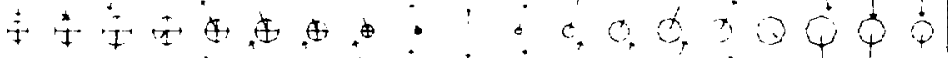

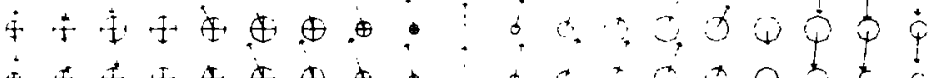

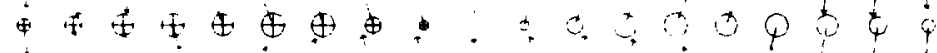

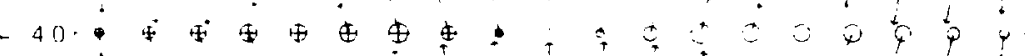
3.5 .

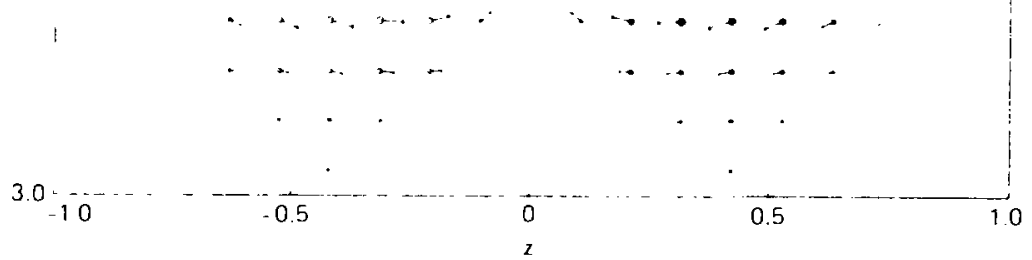

Maximum velocity in plane (arrows) $=1.75713354 \mathrm{E} 02$

Maximum perpendicular velocity (cırcles) $=1.85697924 \mathrm{E} .02$

Figure v. 9h the velocity fiela wn the $=+14$ plane in the $1 / 2$ ideal kink simulation at $t=7 \pm$ Alfven transit times. 


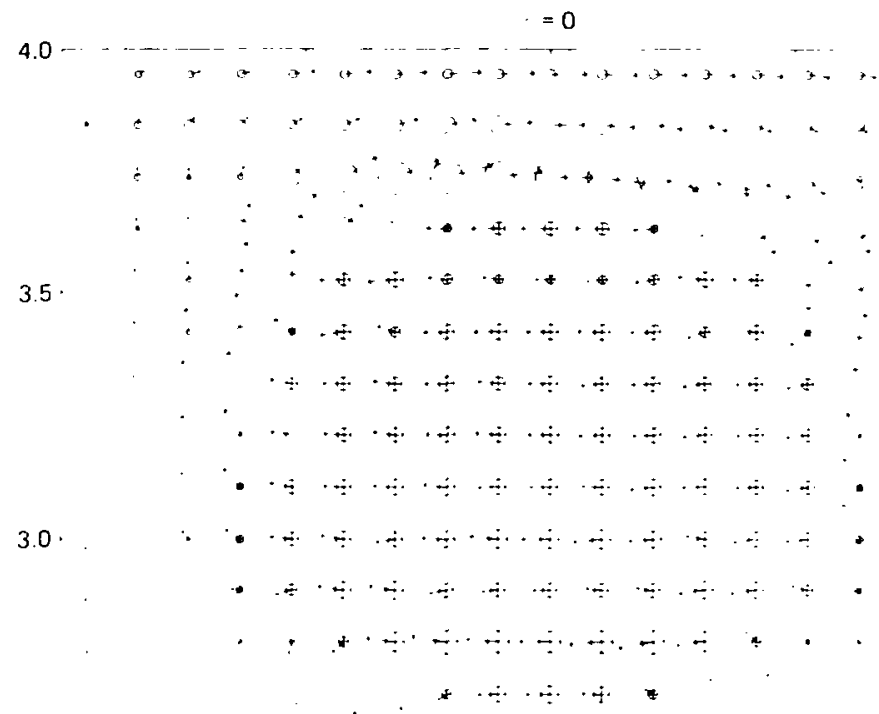

2.5

2.0

$-1.0$

05

D

05

10

Maximum velocity in plane tarrows) $=7.88063238 \mathrm{E} 05$

Maximum per endicular velocity in"cles) $=1.47352917 \mathrm{E} 04$

Figure 1.1 !

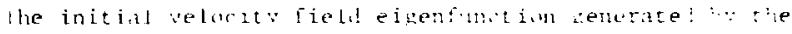
linear cute as projected an the plane. 
$=0$

4.0

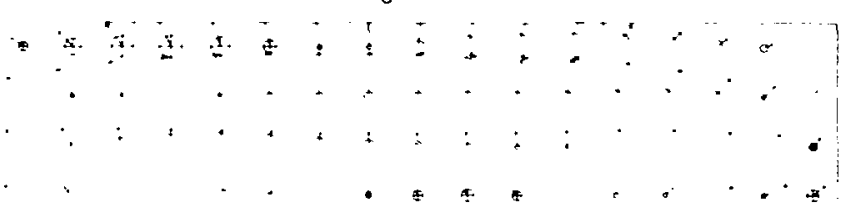

35

- 30

.4

$30 \cdot \therefore$

$\therefore \ldots$

; .

25
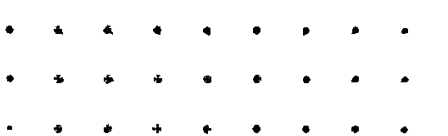


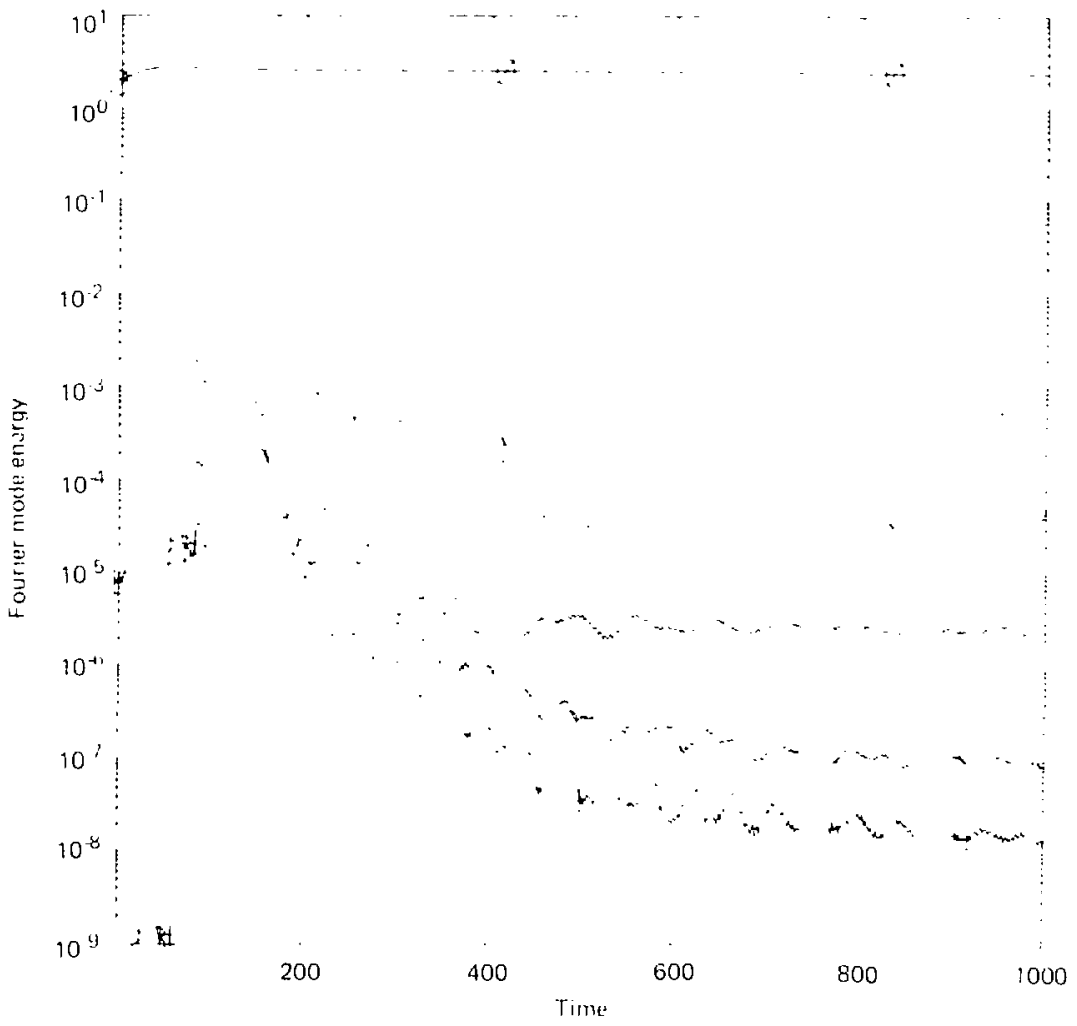

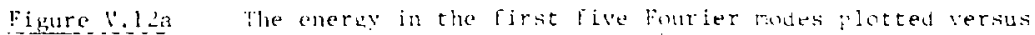

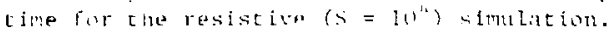



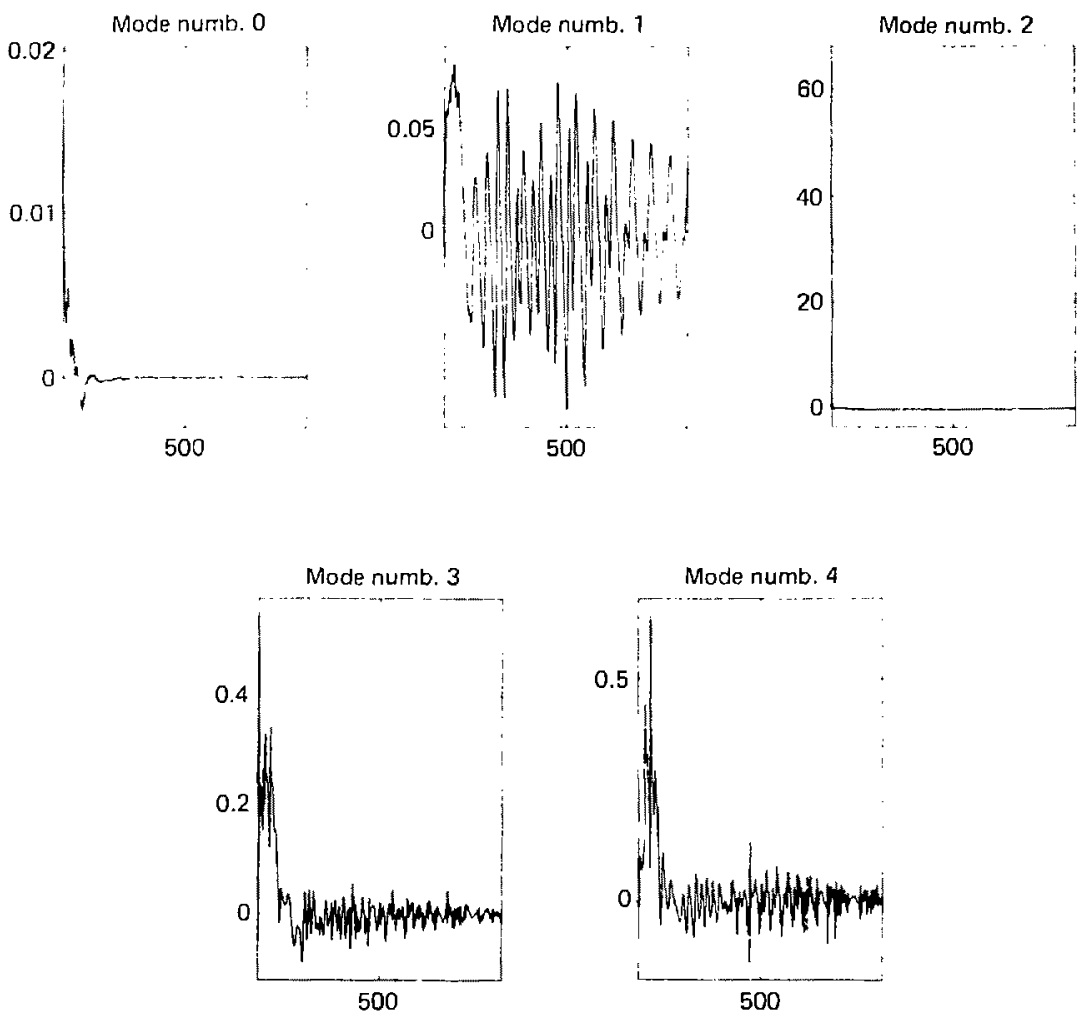

Figure V.12b lhe growth rates $\gamma_{\mathrm{N}}$ for the Fourier modes depicted In Fig. V. 12a. 


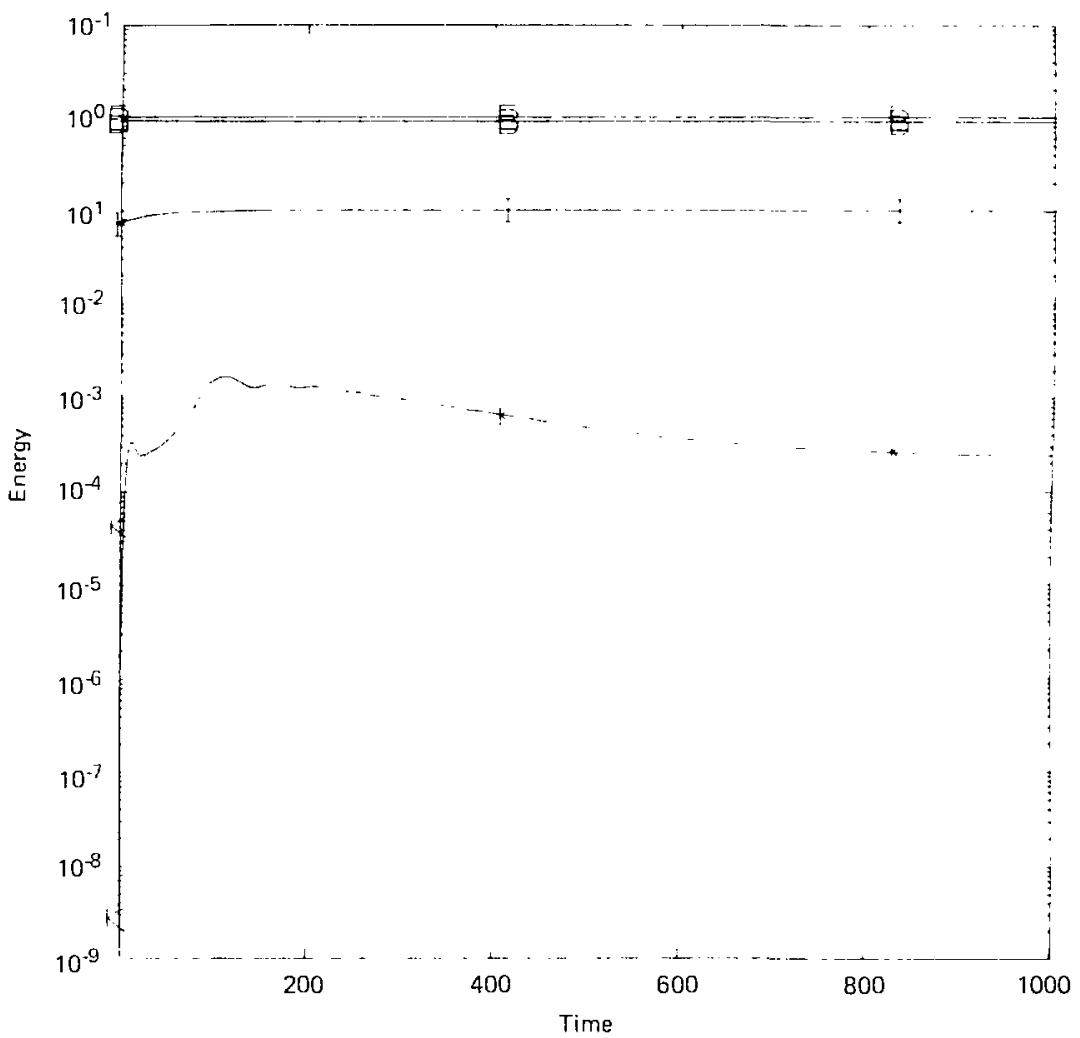

Figure V.13a The kinetic energy plotted versus time for the resistive ( $\mathrm{S}=10^{4}$ ) simulation. 


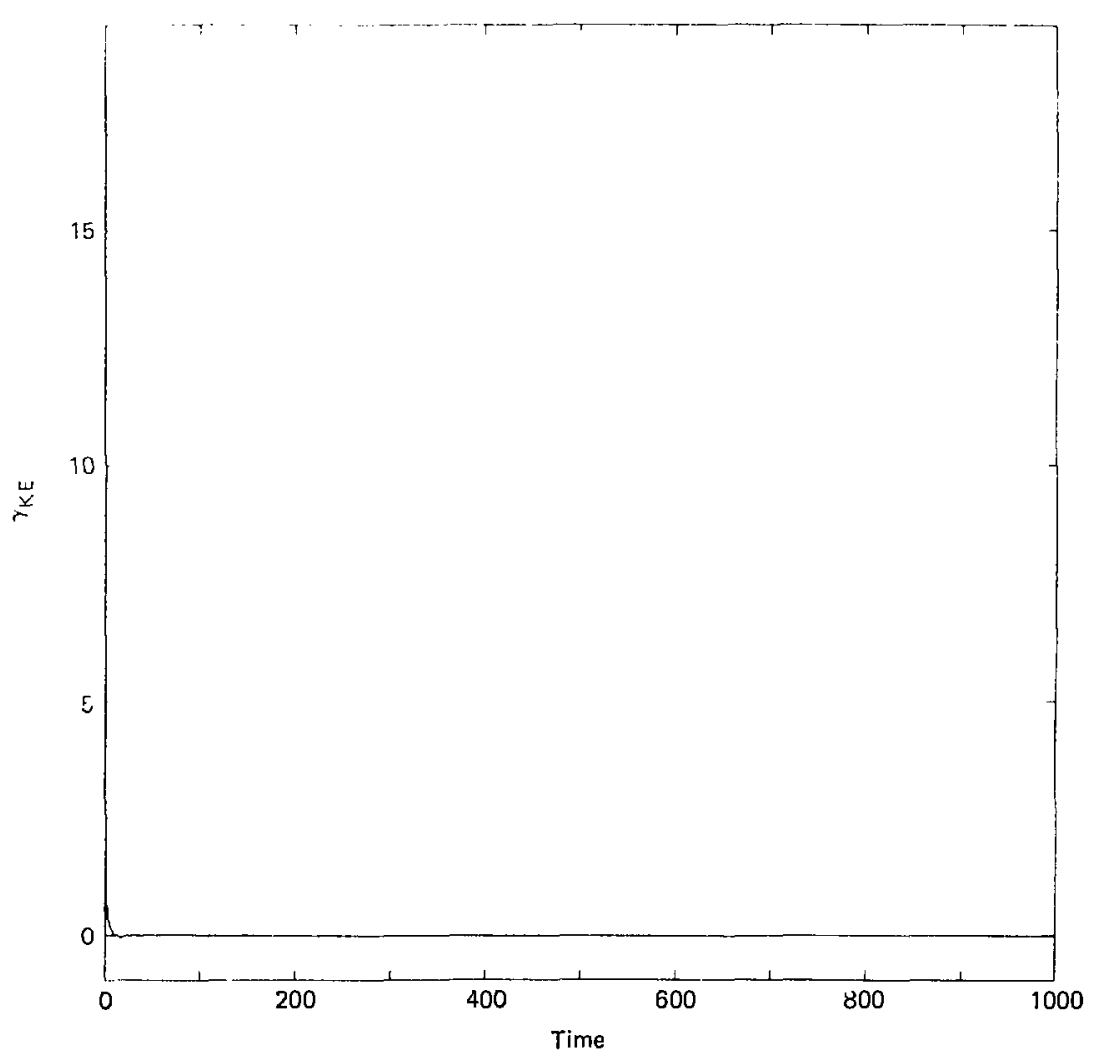

Figure $\because .13 \mathrm{~b}$ Ihe growth rate $\gamma_{\mathrm{KL}}$ calculated from the kinetic energy in Fig. V.l2a. 

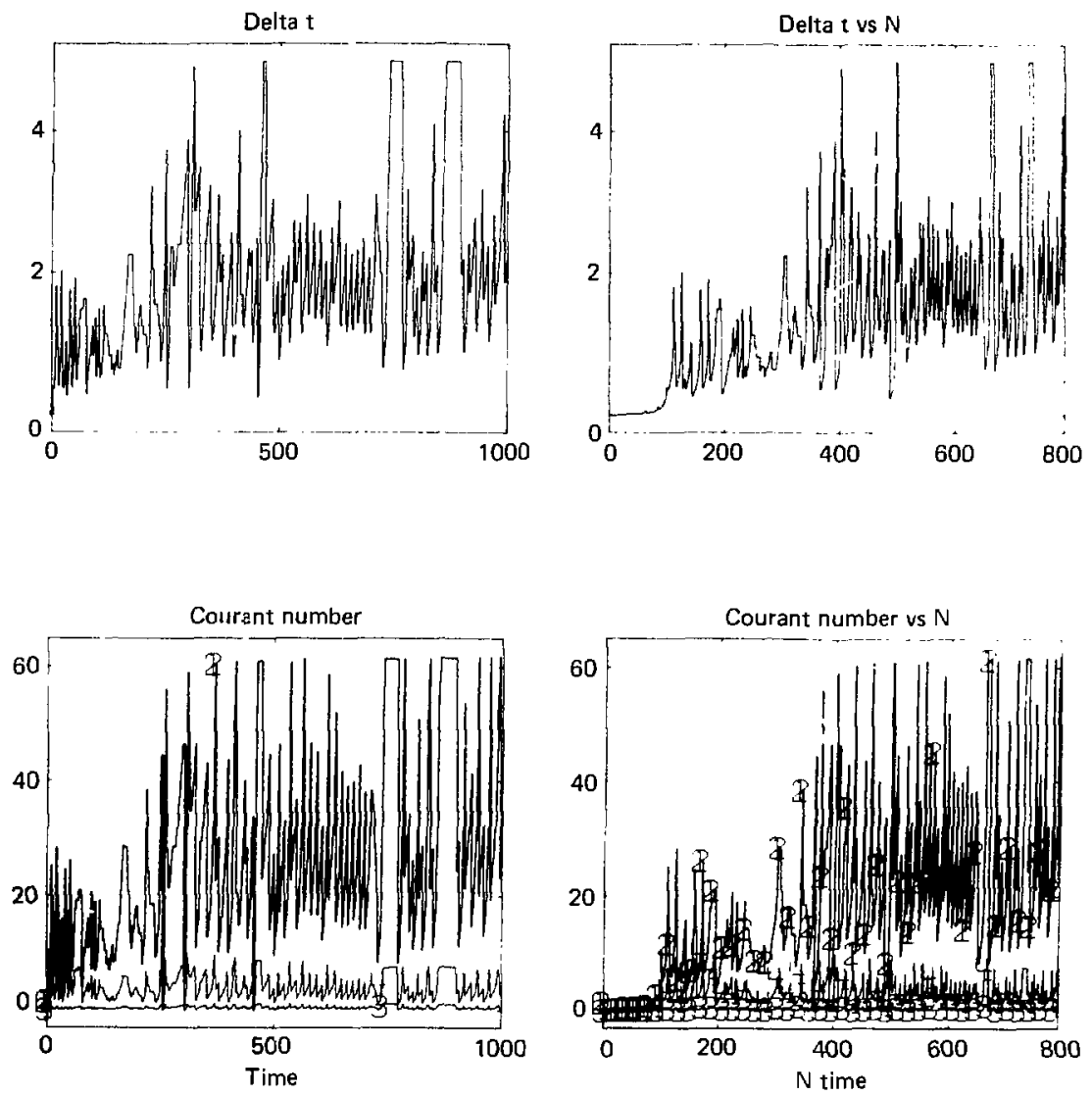

Figure V.14

The timestep size used plotted versus a) time; and

b) timestep number. Also, the Courant number to which these $\Delta t^{\prime} s$ correspond plotted versus c) time, and d) timestep number. 


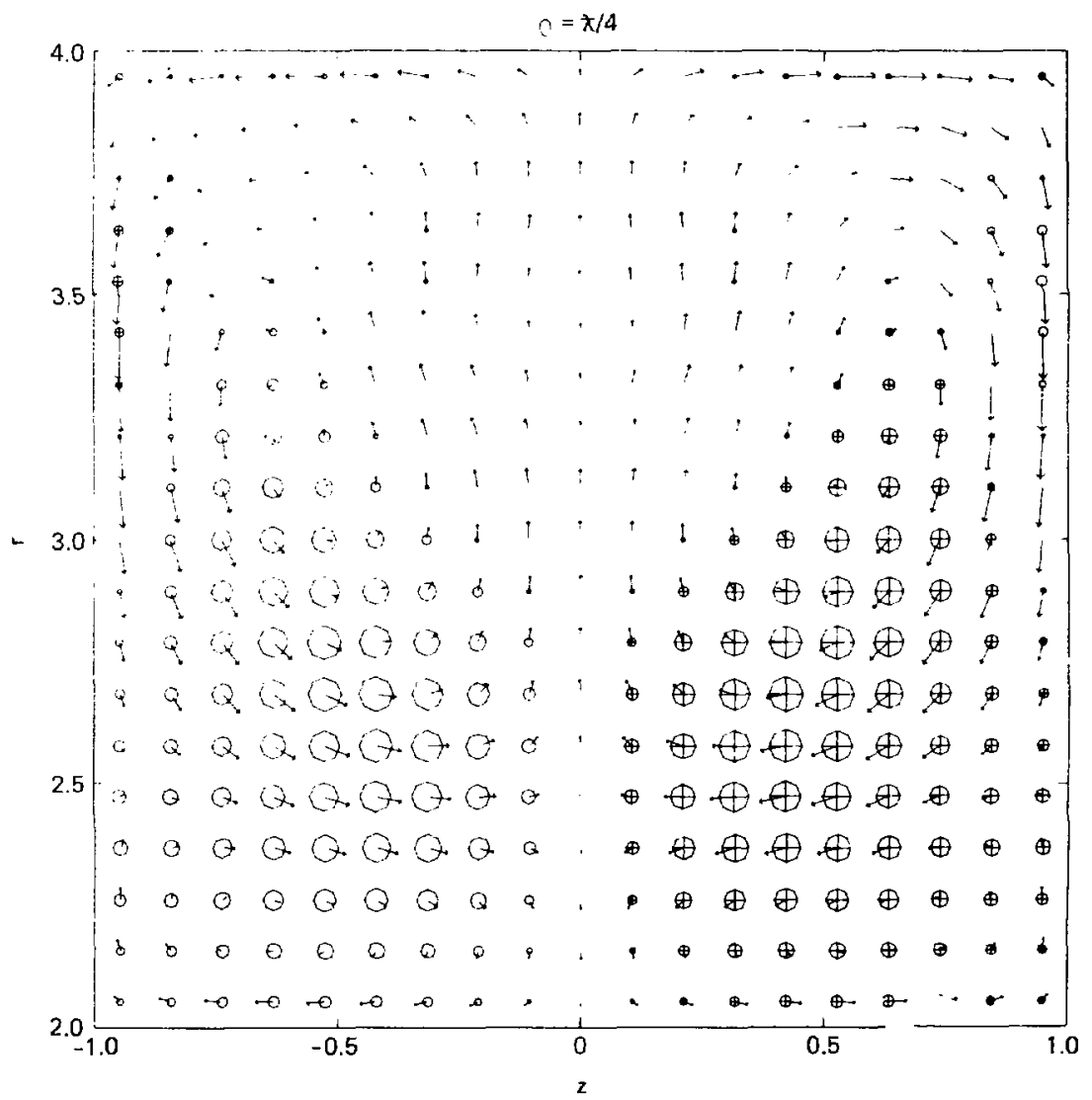

Maximum velocity in plane (arrows) $=1.76190346 \mathrm{E} \cdot r$ :

Maximum perpendicular velocity (circles) $=4503367^{-}$

Figure V.1b

The velocity field on the $1=\lambda /$ plane at $t=58$ Alfuen transit times. The twin counterrote ${ }^{-}$ng vorticies characterlstic of the $m=1$ mode are once again visiole. 


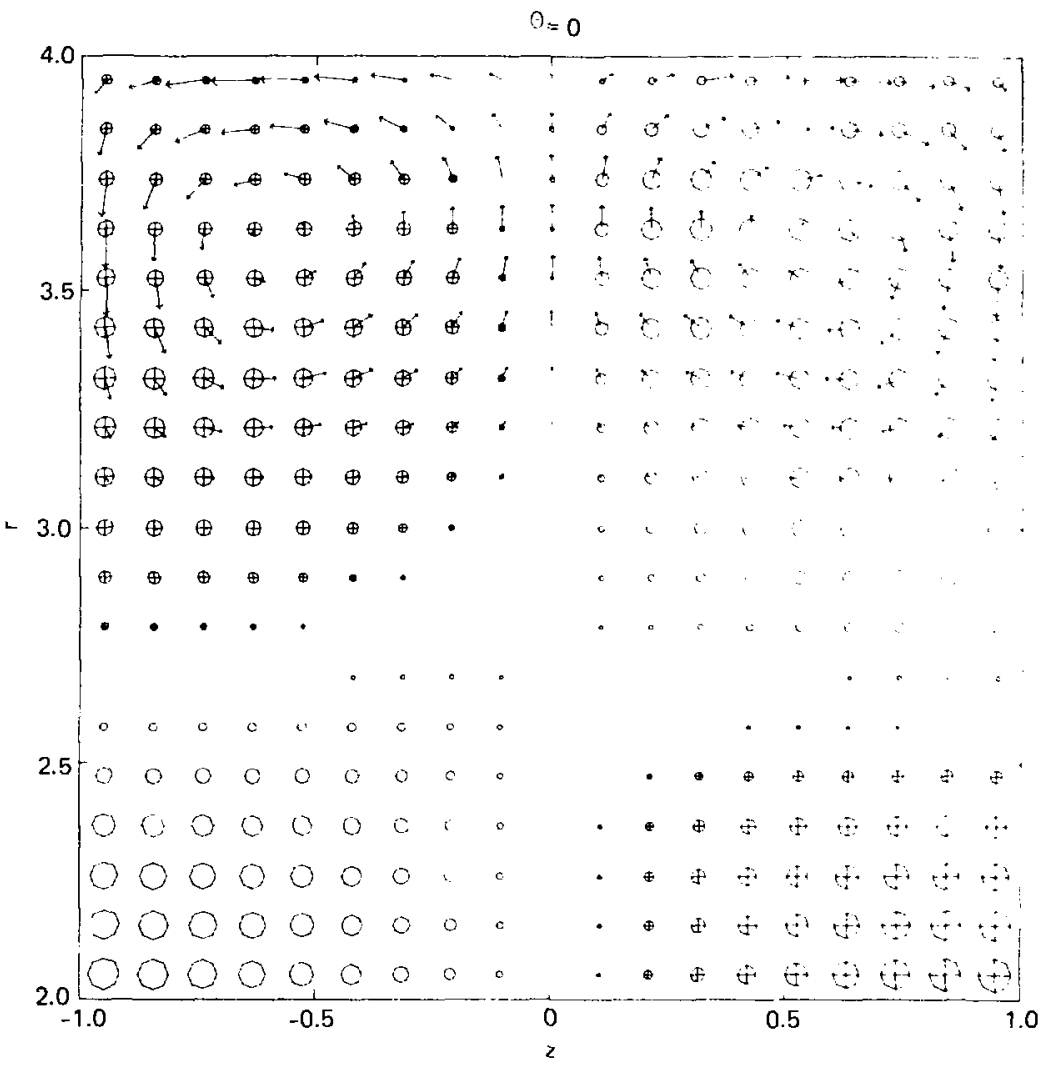

Maximum velocity in plane (arrows) $=4.31998799 \mathrm{E}-02$

Maximum perpendicular velocity (circles) $=1.64773827 \mathrm{E} \cdot 02$ 
$0=\lambda / 4$

4.0

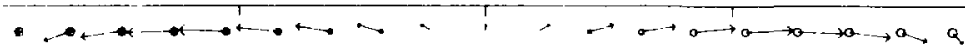

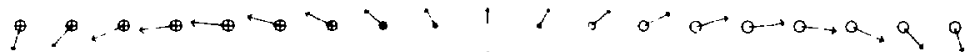

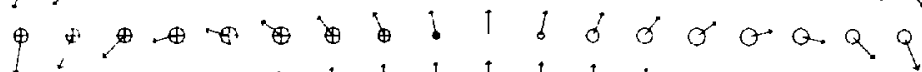

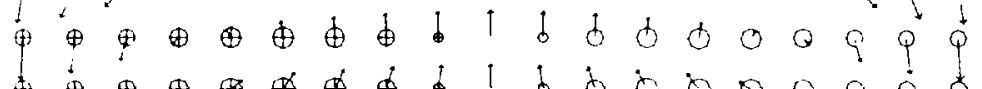

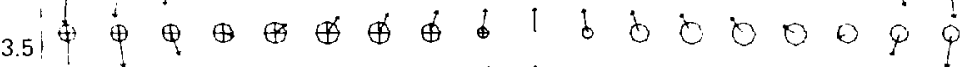

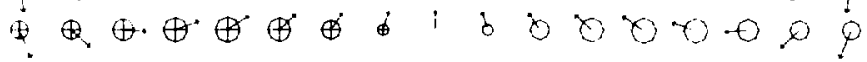

(i) $\$$ स.

.

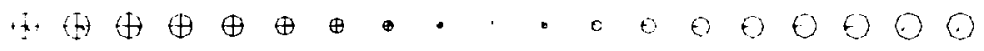

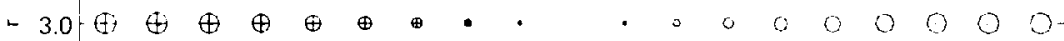

$\oplus \oplus \oplus \oplus \oplus \oplus \cdot$

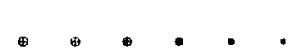

$2.5\left[\begin{array}{lllllll}0 & 0 & 0 & 0 & 0 & 0 & 0 \\ 0 & 0 & 0 & 3 & 0 & 0 & 0\end{array}\right.$

$0000000 \% \oplus \oplus \oplus \oplus \oplus \oplus \oplus \oplus$

$0000000 \circ \oplus \oplus \oplus \oplus \oplus \oplus \oplus \oplus$

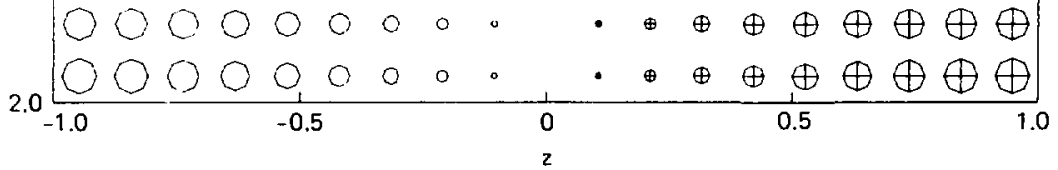

Maximum velacity in plane (arrows) $=4.16385436 \mathrm{E} \cdot 02$

Maximum perpendicular velocity (circles) $=1.59099000 \mathrm{E}-02$

Figure V.16b The veloctty field at $t=1000$ Alfven transits on the $\theta=\lambda / 4$ plane. 
$\theta=0$

4.0

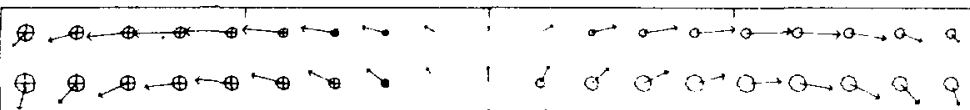

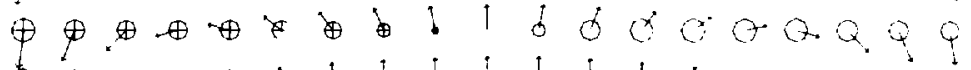

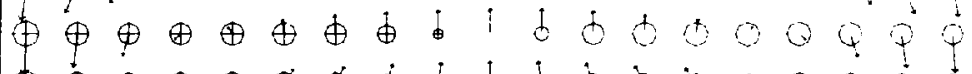

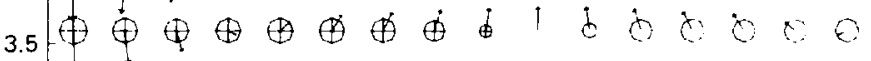

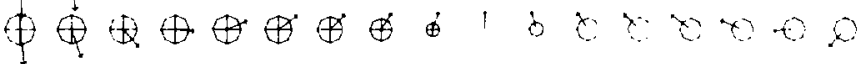

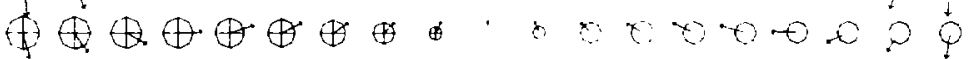

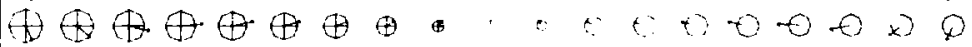

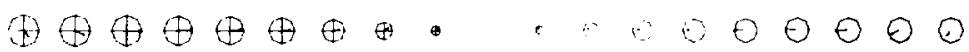
$-3.0 H \Theta \oplus \oplus \oplus \oplus \oplus \oplus, \cdots \cdots 00000$

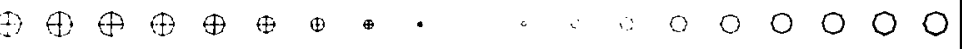
(千;

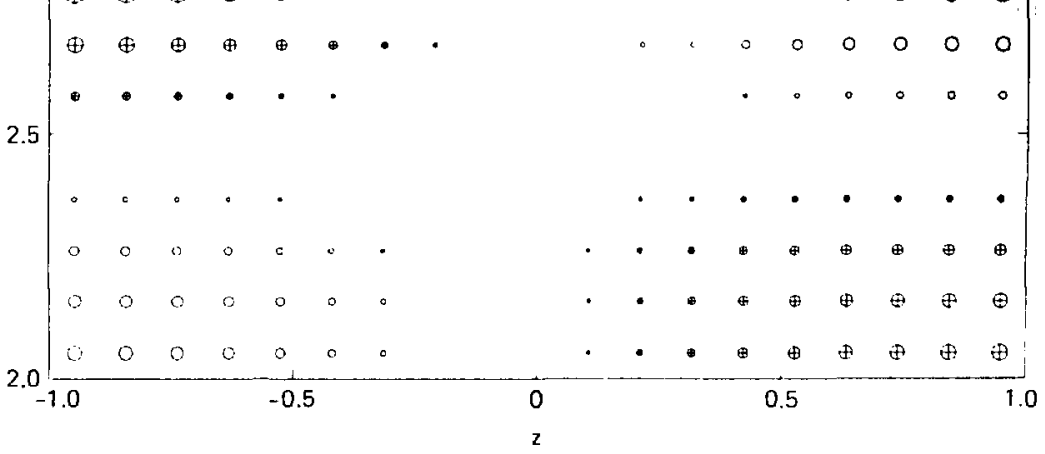

Maximum velocity in plane (arrows) $=4.37184582 \mathrm{E}$. ?

Maximum perpendiculai velocity (circles) $=1.328853 \quad$ ‥02

Figure V.17a The velocity field at $t=1500$ Alfvén transits on the $0=0$ plane. 
$4.0 \quad \theta=\pi / 4$

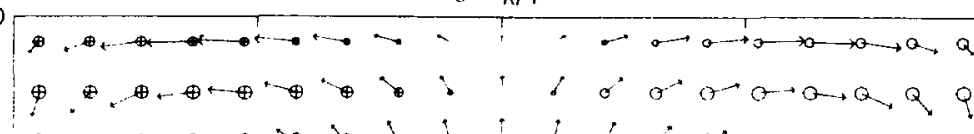

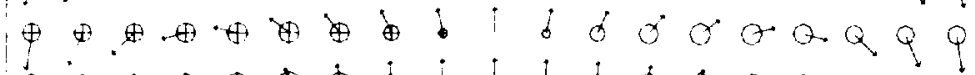

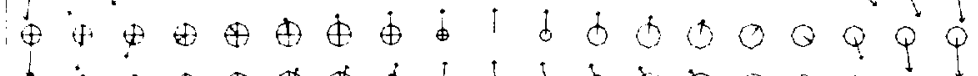
3.5. स. fi

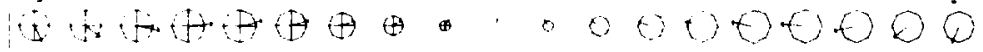

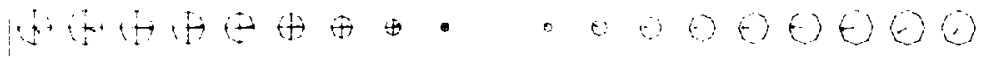

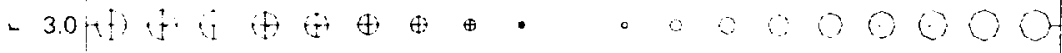

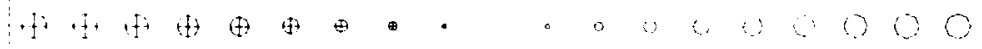

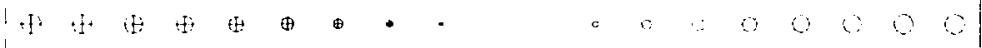

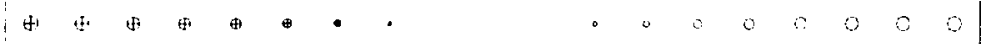
2.5

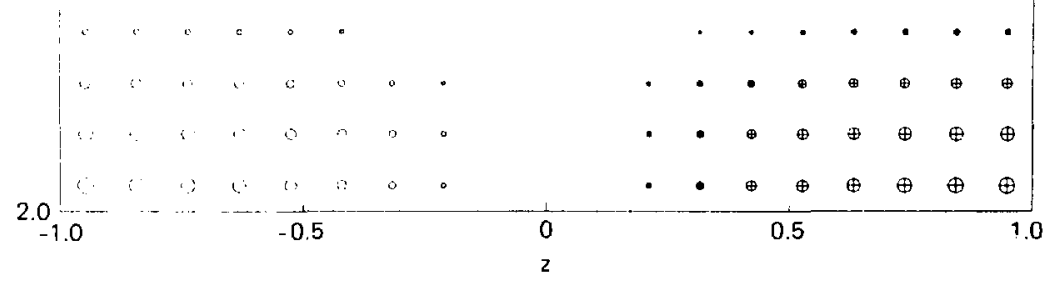

Maximum velocity in plane (arrows) $=4.20433193 \mathrm{E} \cdot 02$

Maximum perpendicular velocity (circles) $=1.32444421 \mathrm{E}-02$

Figure V.17b The velocity fleld at $t=15 i^{\circ}$ Alfvén $t$ :anstes on the $0=t / 4$ plane. 
CHAPTER VI

SPONTANEOUS REVERSED FIELD PINCH

\section{VI.1 Introduction}

The spontaneous production of a reversed toroidal field in pinches in which the toroidal flux is conserved has been observed in the ZETA and HBTX experiments. ${ }^{72}$ Taylor ${ }^{73}$ has shown that this is a relaxation to a minimum energy state of a moderately resistive plasma subject to the constraint that

$$
K=\int_{V} \underline{A} \cdot \underline{B} d \tau
$$

be a constant ( $\underline{A}$ is the vector potential, $\underline{B}$ is the magnetic flux densitr, and $V$ is the total plasma volume). The final plasma state then satisfies the rondition

$$
\nabla \times \underline{B}=\mu \underline{B}
$$

where $\mu$ is a constant throughout the plasma. This final stace is terme "force-free" due to the vanishing of the Lorentz force term $(\underline{x} \underline{x}=0)$. Stable final states can exist for plasmas with pinch ratios $x \leq A^{-1}$ (the aspect ratio) and $x \geqslant 1$. The pinch ratio $y$ is defined as

$$
x \equiv \frac{2 I}{r_{0} B_{0}}
$$

where $I$ is the current in the plasma; $B_{0}$ is the initial tcroidal magnetic 
field; and $r_{0}$ is the minor radius of the torus. In a Cartesian geometry, the pinch ratio may be defined as

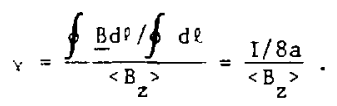

The spontaneous reversal of the torotdal field is demonstrated by three simulations performed in Cartesiar coordinates. In the first, the 'valution of a high current equilibrium is traced. The plasma undergoes a very quickly growing $m=1, \pi=1 \mathrm{kink}$ that leads to field reversal on the boundary. This is in agreement with the result obtained by Pritchett. 74,75 In the second and third cases, low currext density plasmas are subjected to an external electric field tha drives the plasma into the $x ; 1$ regine. In chese cases, a strong field reversal occurs. The reversal then decays on the resistive timescale. The temporal variation of the current, kiretic energy and field reversal are in good qualitative agreement "itl the work of sykes and Wesson, ${ }^{45}$ and Gowers, et al. ${ }^{46}$ One important difference in our physical model as opposed to that of sykes and Wesson is that we obtain the field reversal using a scalar resistivity while they use a tensor model.

\section{VI.2 Initial Conditions}

The equilibcium specified in Eq. IV.2.2 is used for all three simulations in this chapter. The high i: itial $x$ simulation (Case 1) is characterized by a $q=.15, x_{0}=2.53$, and $t=1$. It is perturbed by the velocity vortices described by Eq. IV.3.1,2 with $c=10^{-4}$. The initiel plasma state is shown in Fig. VI.1.

The first low inftial $x$ simulation (Case 2) is characterized by $\mathrm{q}_{0}=.8, \mathrm{x}_{0}=.98$ and $t=.5$. The second simulation of low $\lambda$ (Case 3 ) 
has $\mathrm{q}_{0}=1.1, x_{0}=.72$ and $t=1$. The perturbations for these cases are ger zed by the linear code and are added to the equilibrium configuration using Eq. IV. 3.3 with $C^{\prime}=.01$.

\section{3 Boundary Conditions and Zoning}

The adiabatic, perfectly conducting wall boundary cenditions (set Chapter: IIT and IV) are once again used, as are the scale factors giver ir Eqs. IV. 4.2 and IV.4.3.

\section{'T. 3.1 Case 1}

Since we are running at high currents, the singular laver $(\underline{k} \cdot \underline{B}=0)$ is close to the wall. In order to keep the number of mest. points to a minimum and yet to resolve any large functional var iationi

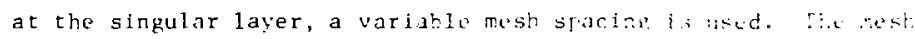
spacings are chosen by usirg a geometric ratio, $R$, to connect the pone siz:s by

$$
\frac{x_{i+1}-x_{i}}{x_{i}-x_{i-1}}=R \text {. }
$$

The ratio is chosen so that the zones near the wall are approximately ten times smaller than the central zones. This is also a check on the nonuniform zoning capabilitips in IMP.

\section{3.2 Case 2 and Case 3}

In order to impose an external electric field, a time dependent boundary condition is required. The conservation of magnetic flux 
equation (II.2.12.a) is written as

$$
\frac{B}{i t}+x E=0
$$

wite $\mathrm{re}$ in a normalized electric field. For the poloidal field this hecomes

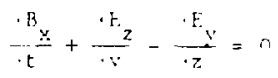

$a n+1$

$$
\frac{\mathrm{B}}{\mathrm{l}} \dot{\mathrm{l}}+\frac{\cdot \mathrm{x}}{\cdot z}-\frac{1}{\cdot \mathrm{x}}=0
$$

The normal value for tho tangential telectic field at che houndar: a atru since the ball is assumed to be a perfect conductor. in nom lot che tangential eleciric field at the biall ha.

$$
\underline{E}=F_{z}(t) i \text {. }
$$

l'sing the spatial finite differences approximations given in chapter III, we now write (VI, 3.3) as

$$
\frac{B_{x}}{3 t}+\frac{\bar{E}_{z}(y+)-\ddot{E}_{z}(y-)}{2^{L}}=0
$$

and 


$$
\frac{B_{y}}{i t}-\frac{\bar{E}_{z}(x+)-\bar{E}_{z}(x-)}{1^{2}}=0 \text {. }
$$

Depending on which boundary we are at, the appropriate $\bar{E}_{z}$ is given the valie of $E_{z}(t)$. The $t$ ime dependent electric field in he $z$ direction is specified by

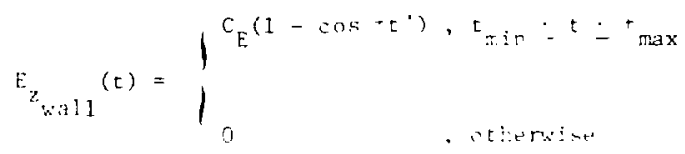

wher.

$$
\begin{aligned}
& -\frac{1-}{t_{\operatorname{mix}}-t_{\min }}, \\
& t^{\prime} t-t_{\min },
\end{aligned}
$$

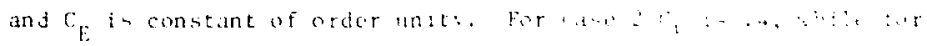

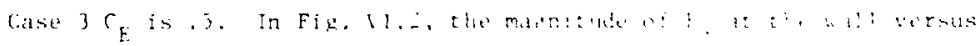
cime is shown in wits of c $F$

\section{VI.4 Artifizial Viscosity}

The electric field shown in Fig. l'I. I cames an increasie in tw" current flowing in the plasma, thus pinching the plasm. Tho pinctine causes a compression of the plasma that leads to numerid difficulties. These cif iculties are resolved by the int umion of a ban xeumann $5 n$ artificial viscosi-y in the momentum and entrov equations. In the nomen- 
clature of Chapter III, a viscous flux is added to the momentum equation,

$$
\frac{\partial \rho \underline{v}}{\partial t}+\nabla \cdot(\underline{\underline{v}} \underline{\underline{v}}+\underline{q})+\underline{B} \times(\nabla \times \underline{B})+\frac{1}{2} \nabla P=0 .
$$

and a viscous or shock heating fonce is added to the energy equatior

$$
\frac{\partial P}{\partial t}+\gamma \nabla \cdot \underline{P} \underline{v}-(\gamma-1) \underline{v} \cdot \nabla P+\frac{2 \eta}{S} j^{2}+(\underline{g} \cdot \nabla) \cdot \underline{v}=0
$$

where

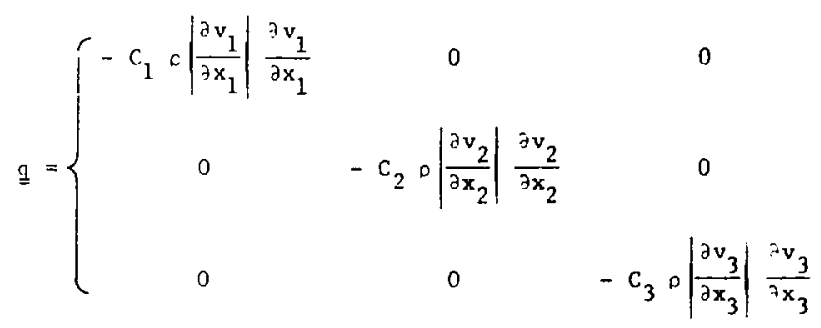

and

$$
c_{i}= \begin{cases}c_{7}, \frac{\partial v_{i}}{\partial x_{i}}<0 ; i=1,2,3 \\ 0, \text { olherwise }\end{cases}
$$

Thus, the artificial viscosity only effects compression and does not effect rarifaction. Typical values of $\mathrm{C}_{\mathrm{q}}$ are .5 .

\section{5 Results}

VI.5.1 Case 1: High Initial \%

In this case, as in all cases presented in this chap:er, the plasma is allowed to react to the effects of resistive diffusion and 
ohmic heating ( $\mathrm{S}=250$ in this case) by satting the external electric field and the pseudo cooling terms in the magnetic field and pressure equations to zero. This causes an immediate flow of flasma toward the walls. The kinetic energy exhibits rapid growth in the first few tenths of an Alfven transit due to these effects (Fig vI.3). Once this flow is established, the kinetic energy remains relatively constant until an $m=1, n=1$ mode begins to dominate the problem. The mode grows exponentially from $t=2$ to $t=8.5$. At $t=8.5$, the $m=1, n=$ 1 mode saturates whlle an $n=$; mode continues to grow until it saturates at $t=10$. This can be observed in the plots of the energy in the Fourier modes given in Fig. VI.4. The late time reversal of the toroidal magnetic field is illustratej in Fig. VT. 5 which shows the $\mathrm{B}_{z}$ field on a number of lines acrnss tlie mesh. The original value for $B_{2}$ was 1 everywhere, while these late time plots show $B_{2}$ difning to values of -2 in some positions.

\section{VI.5.2 Case 2: Externally Applied Electric Field, $\mathrm{q}_{0}=.8$}

The plasma iritially displays the flows caused hy resistive diffusion and heating ( $S=50$ in Cases 2 and 3 ). These effects are first balanced and then overcome by the effects of the impressed electric field at the boundaries. The velocity field during the pinch phase is given in Fig. VI.6, while isoplots ó the initially uniform density are given in Fig. VI. $i$ wich display circular contours that are caused by the magnetic pinch. Velocity field plots durin? the kinking phase of the simulation are shown in Fig. VI.8, while isoplots of the density are given in Fig. VI.9. The flow due to the kink is shown to 
be tightly spirally around the center of the plpe. The pinch ratio rises from an initial value of .98 to a peak of 2.88 (see Fig. VI. 10). This drops rapidly to values of 2.3 , followed by a smaller drop to 2.19. Following these rapid drops, $y$ then slowly falls as the current decays due to the effects of resistivity.

Time history plots of energy and Fourter mode energy (Fig. VI.1I) show growth in kinetic energy due to the extemal electric field from $t=0.5$ to $t=2$. The growth of $k$ inetic energy then ceases while the plasma continues to heat. By $t=4$ transits, the m $=1, n=1$ and the $m=1, n=2$ modes have grown to a level that causes the kinet lc energy to again exhibit growth. At this point, the current contüned in the plasma has peaked and shows a rapid drop from $t=6$ to $t=7.5$. At the At the same time, the average value of $B_{z}$ at the wall, $\left\langle B_{z}\right\rangle$, drops to a value of -.67 , a strong field reversal (see Fig. VI.12). "B then begins a rapid rise back to -.2 at which point the kinetic energy begins growth due to an $n=4$ mode. This stabilizes the rapid decay of the reversed field at $t=10$. For the remalnder of the simulation the field reversal decs, on the resistive diffusion timescale until, at $t=40$, it again passes through zero.

These results are in good qualitative agreement with the simulation of Sykes and Wesson ${ }^{45}$ (SW) espectally since our simulatica did not contr.in as complete a physiral model. Sw included the effects of a tensor resistivity and "radiation" cooling. The tensor resitivity model used by $5 W$ assumed that $n_{1}=2 n_{\|}$. This had the effect of peaing 
the current along the axis of the pinch. We, on the other hand, were able to obtain field reversal without this peaking of the current. The "radiation" cooling terms in SW were a simple model which scaled with pressure and inversely with radius. They had the effect of balancing ohmic heating and dissipating the high temperatures generated during the pinch, allowing the central pressure to drop to its prepinch level.

VI.5.3 Case 3: Externally Applied Electric Field, $q_{0}=1.1$

The initial plasma configuration used here has $x=.72$. The current is driven to a peak $x=3$ at $t=6$ (see Fjg. VI.13). The current then resistively decays until $t=11$. At this $t$ ime, there is a sharper $d r c p$ as the $m=1, n=1$ mode causes a slight field reversal. This is followed at $t=16$ by a sharper drop as the $1 / 1$ mode is again destabilized by interactions with high $n$ modes. Maximum field reversal is now observed (Fig. VI.14) as $\left.\angle B_{z}\right\rangle_{w}=-.18$. This lesser reversal compared to Case 2 is due to the smaller amount of fluid (plasma) contained in the device (the ratio of $P / B_{z}^{2}$ is lower now) initially.

The lag time in $\left\langle B_{z}\right\rangle$ observed here corresponds to that seen $i$. the Gowers report in as much as $\left\langle\mathrm{B}_{z}>\right.$ is: 1) brought quickly to a level near zero; 2) remains here for a number of transit times: and 3) then displays a rapid reversal. followed by decay back to the non-reversed regime.

\section{VI.6 Summary}

We have displayed a number of simulations which exhibit a spontaneous field reversal of a plasma with a large $x$. In the first case, agreement 
with Pritchett ${ }^{74,75}$ is obtalned given a high $x$ equilibrium. In the second and third simulations, qualitative agreement with the work of sykes and Wesson $^{45}$ and of Gowers 46 is obtained while using our more idealized model (scalar resistivity and no radiation cooling).

All these runs were performed at timesteps that exceeded the courant limits by average factors of 5 and peak factors of 40 . 

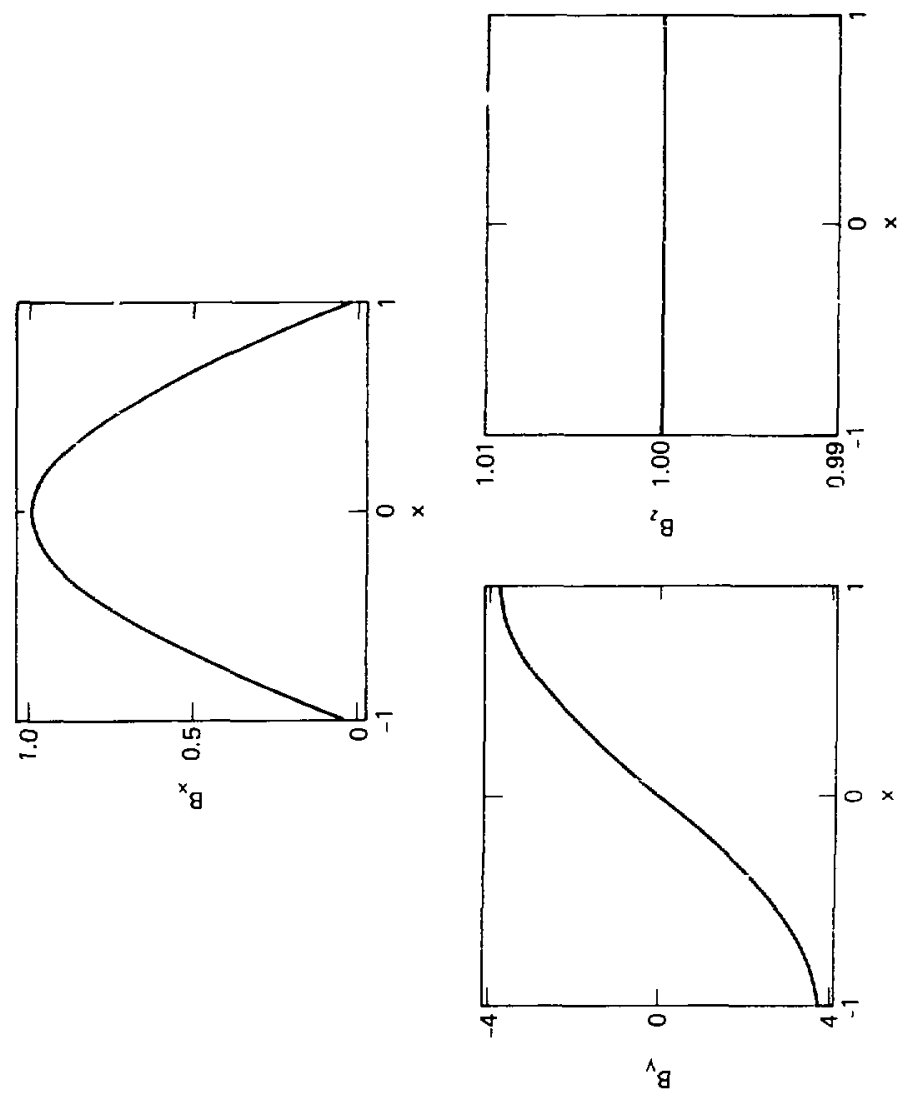

Figure V1.1a

The initial plasma state as viewed across a line $-1 \leq x \leq 1, y=1, z=0$. 
$-190-$

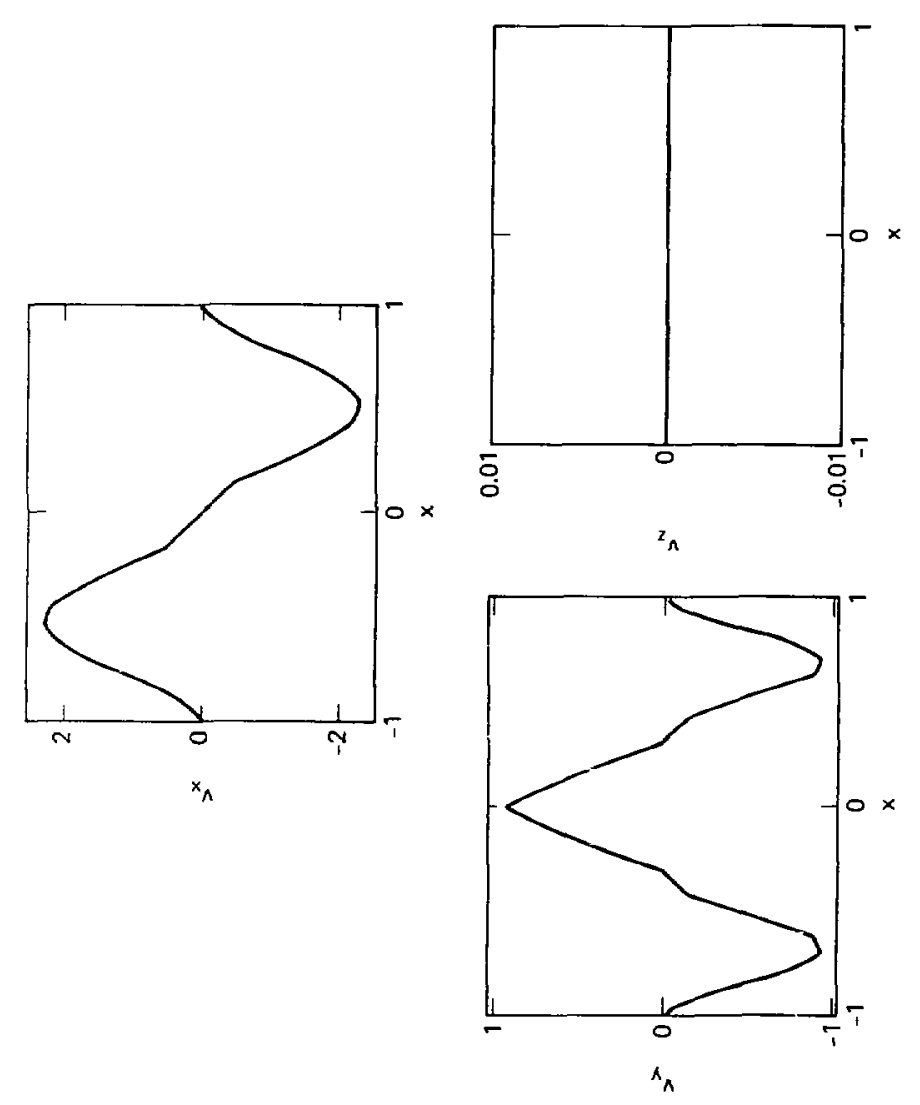

Figure VI.lb The initial plasma state as viewed across a line $-1 \leq x \leq 1, y=1, z=0$. These velocities are the initial perturbation. 
$-191-$
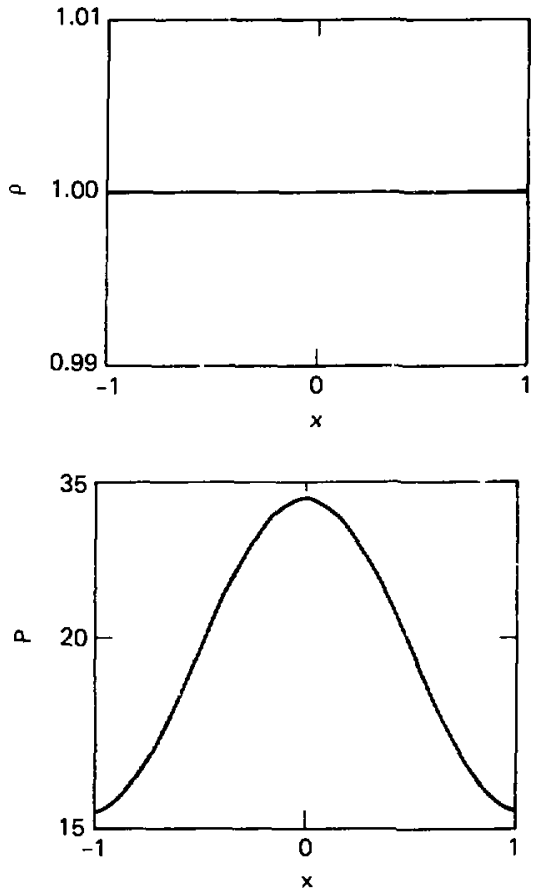

Figure VI.lc The initial plasma state as viewed across a line $-1 \leq x \leq 1, y=1, z=0$. 
$-192-$

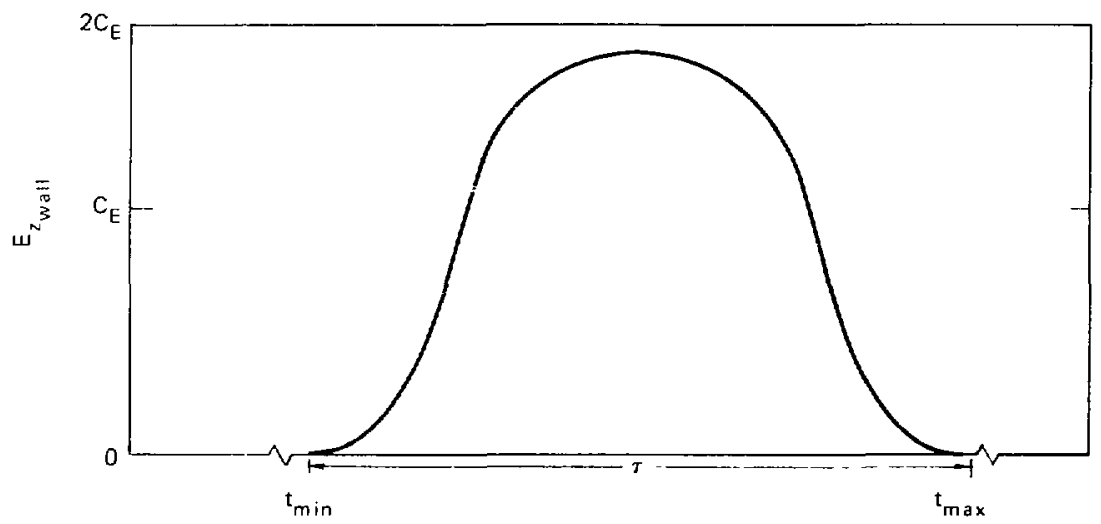

figure VI.2 The time dependant electric field $\mathrm{E}_{2}(t)$ that was used to pinch the plasma $\left(E_{z}(t)=C_{E} 1-\cos \frac{2 \pi\left(t-t_{\text {min }}\right)}{\tau}\right.$ $r_{\min } \leq t \leq t_{\max } ; E_{z}(t)=U$, otherwise). 


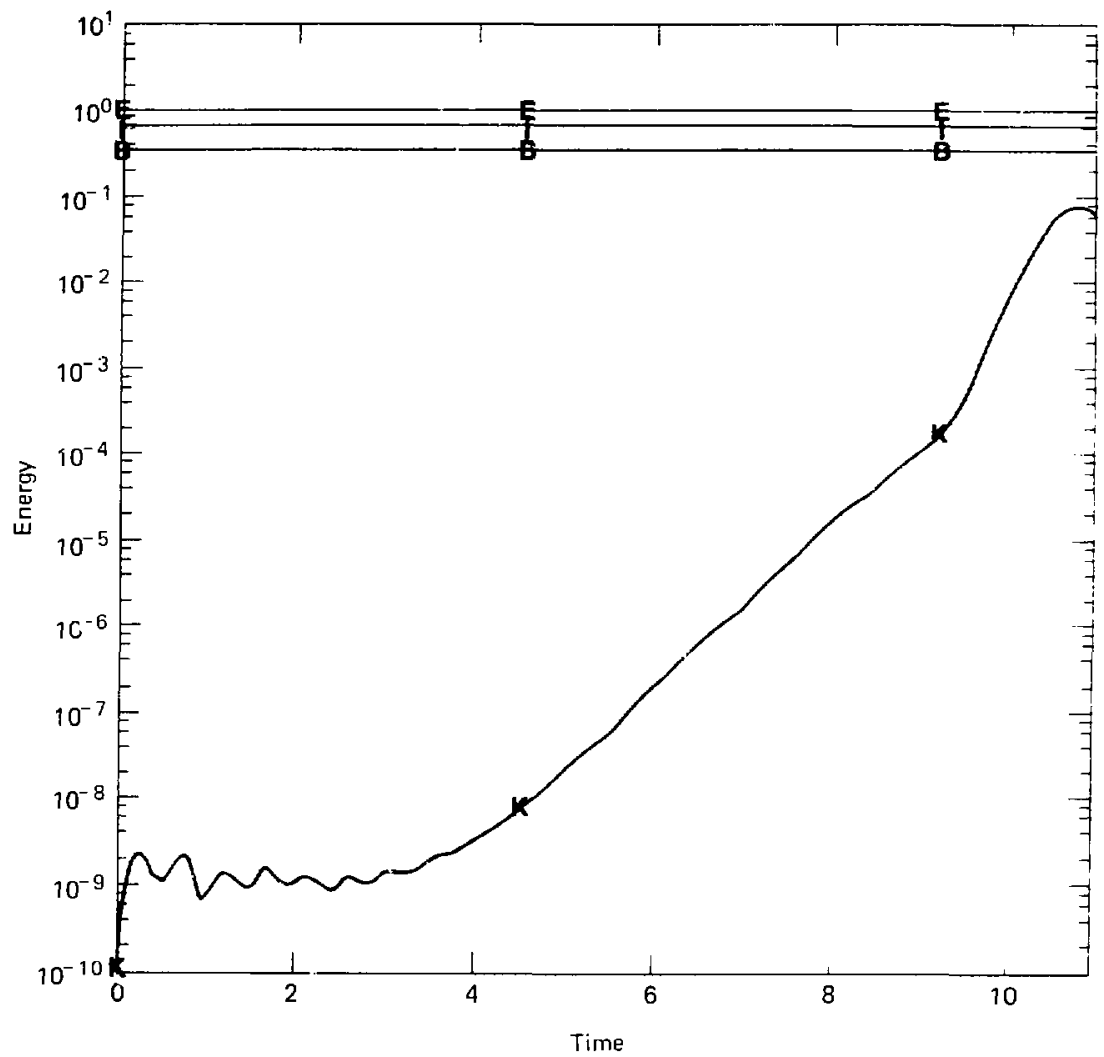

Figure VI.3 The energy versus time plots for Case 1 . 


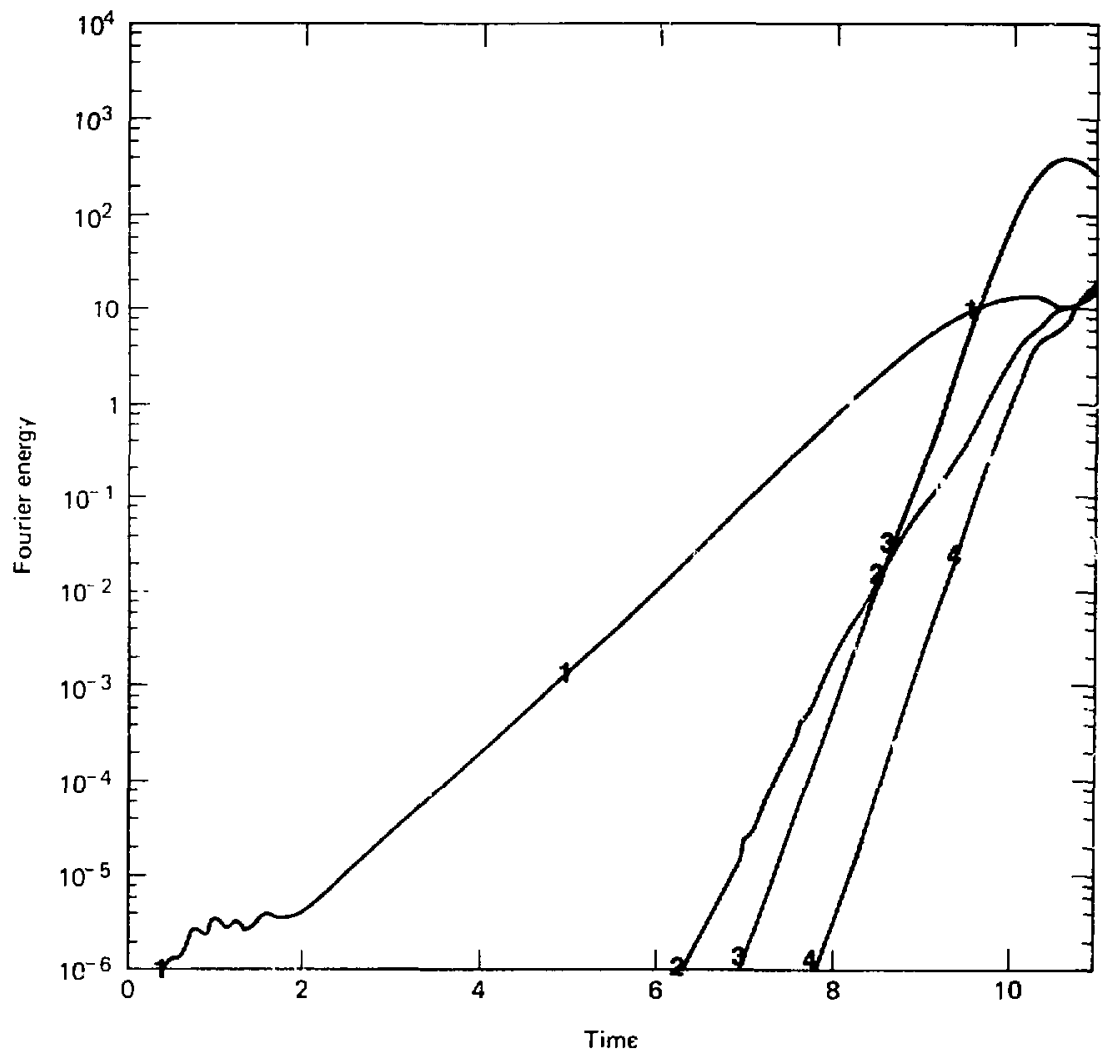

Figure VI.4 The Fourier mode energy plots versus time for Case 1. 

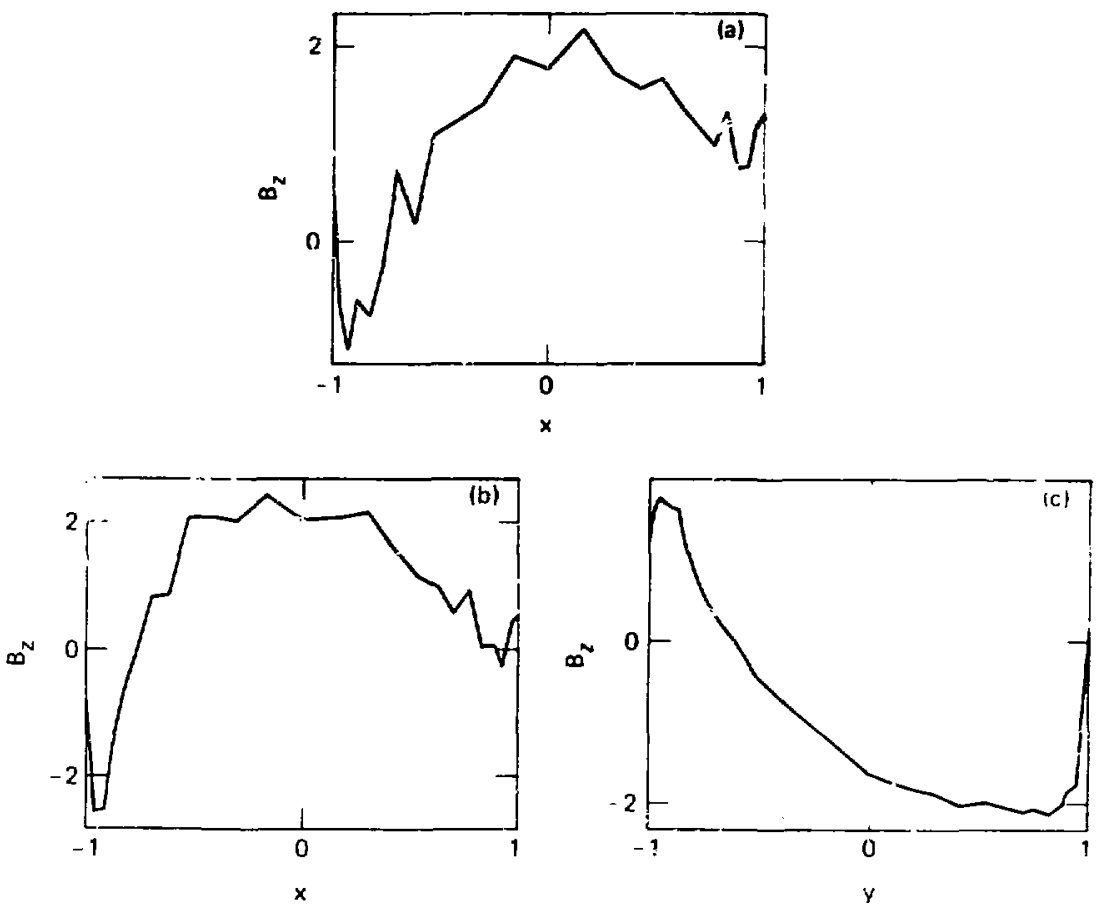

Figure VI.5 Plots of $B_{z}$ versus distance across the grid in Lin

$=0$ plane for Case 1 . Shown are:

e) $-1 \leq x \leq 1, y=-.76 ; \quad$ ) $-1 \leq x \leq 1, z=n ; \neg \pi 1$

c) $x=-.98,-1 \leq y \leq 1$. 


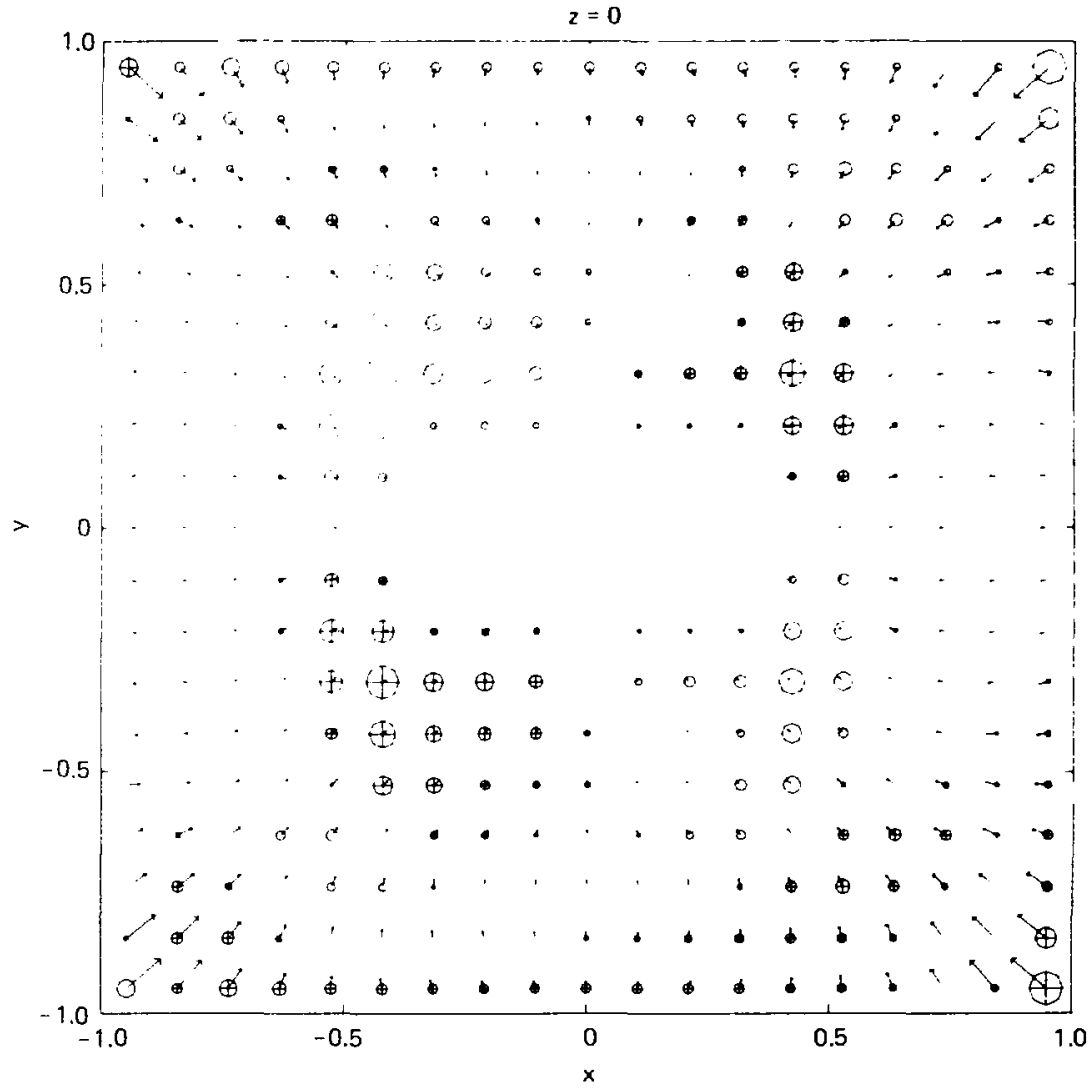

Maximum velocity in plane (arrows) $=1.05049533 \mathrm{E}+00$

Maximum perpendicular valocity (circles) $=2.66125935 \mathrm{E} \cdot 02$

Figure VI.6 The velocity field during the pinch phase of Case 2 and 3. The plasma is being compressed toward the cer:er of the $g^{\top} d$. Only the magnetude of the velocity varys between tile two cases, not its character. 


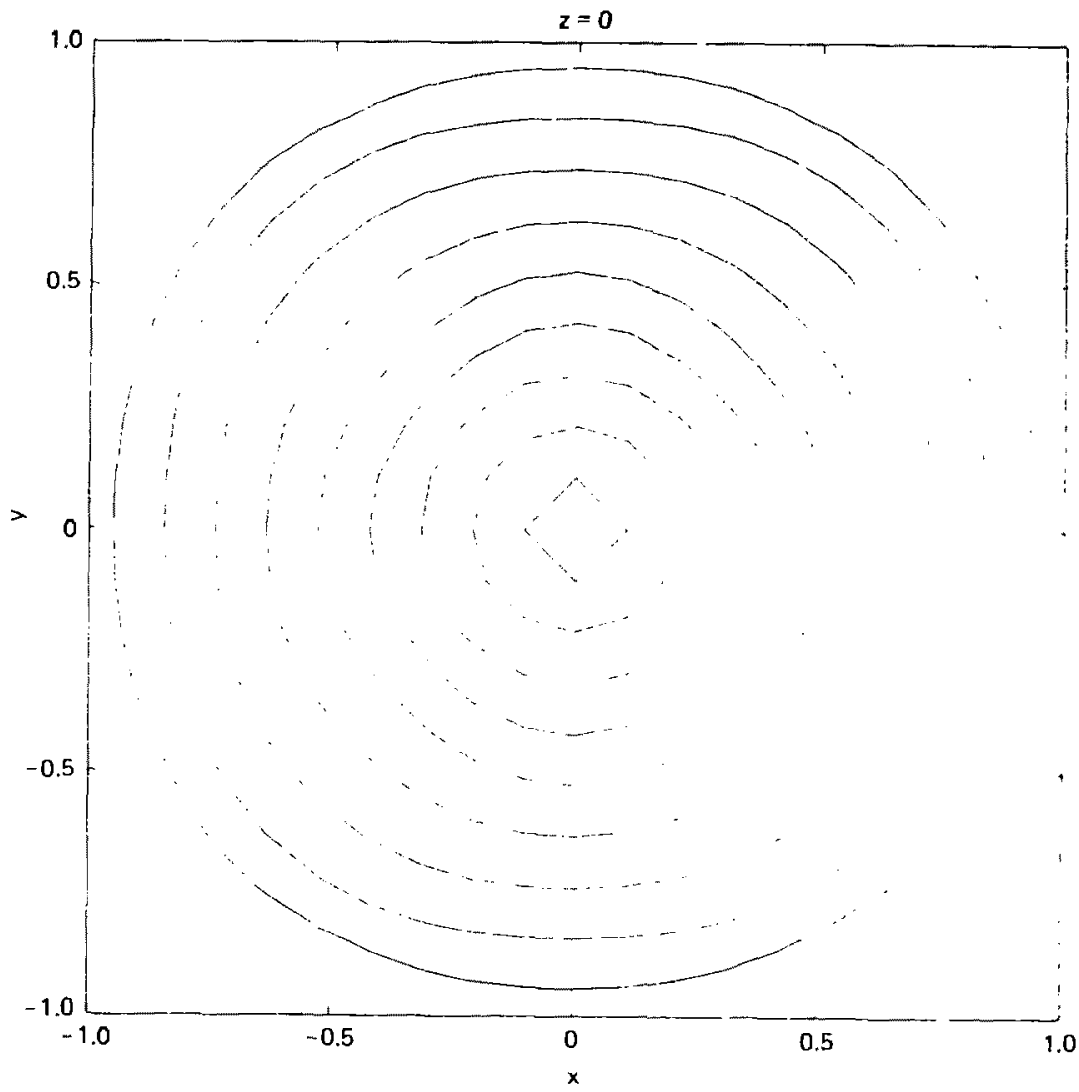

Figure V1.7

1soplots of the density for Cases 2 and 3 during the pinch phase of the simulation. Thile the actual values of contours may vary between the two cases, the circular topology is unchanged. 


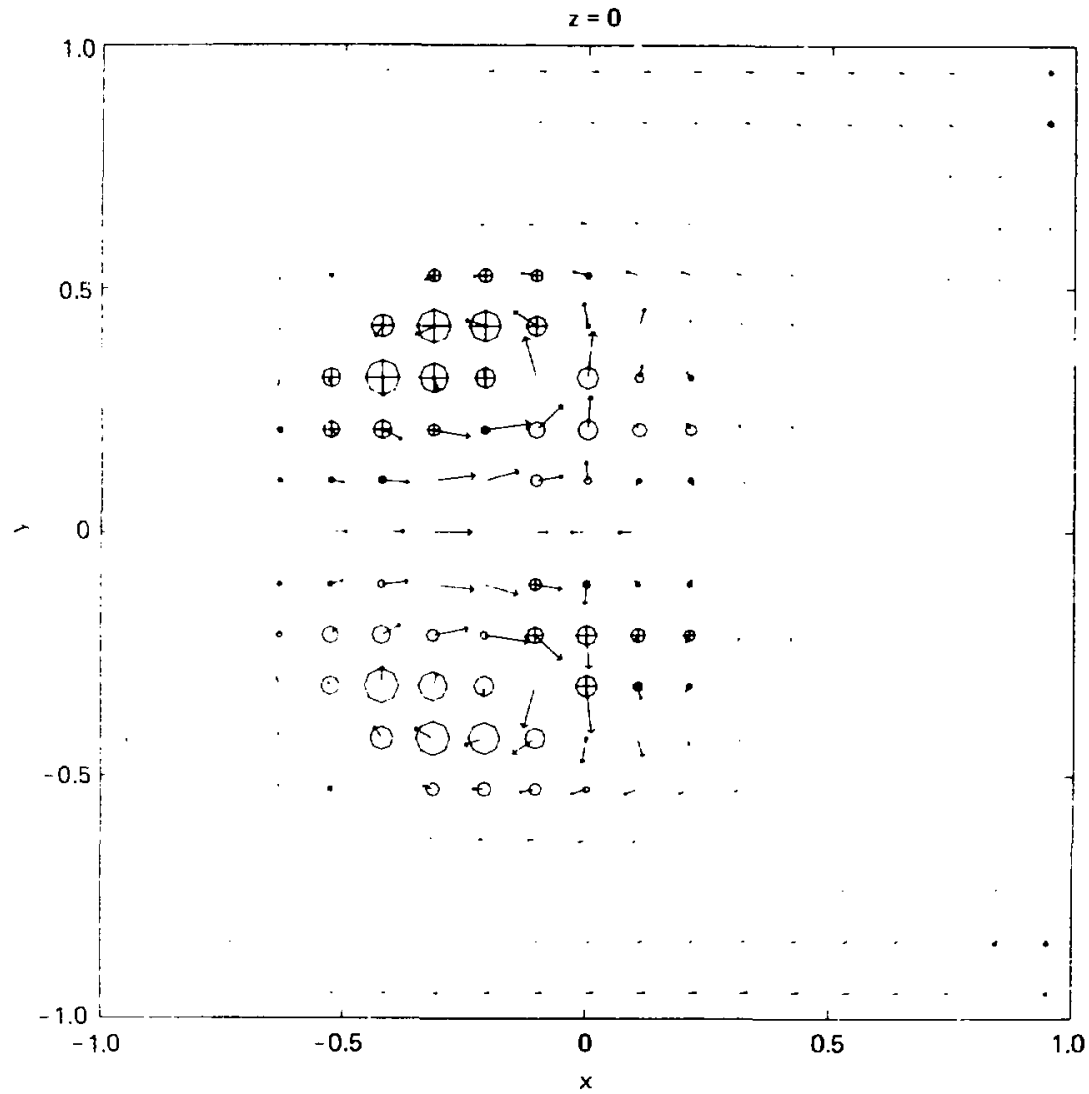

Maximum velocity in plane (arrows) $=7.58314778 \mathrm{E}-01$

Maximum perpendicular velocity (circles) $=2.66125935 \mathrm{E}-02$

Figire VJ.8 The velccity field on the $z=0$ plane at $t=9.4$ Alfven transits into the simulation. Hote that the characteristic $\mathrm{m}=1$ counterrotating vortices have now reappeared but are tightly wound near the center of the mesh. 


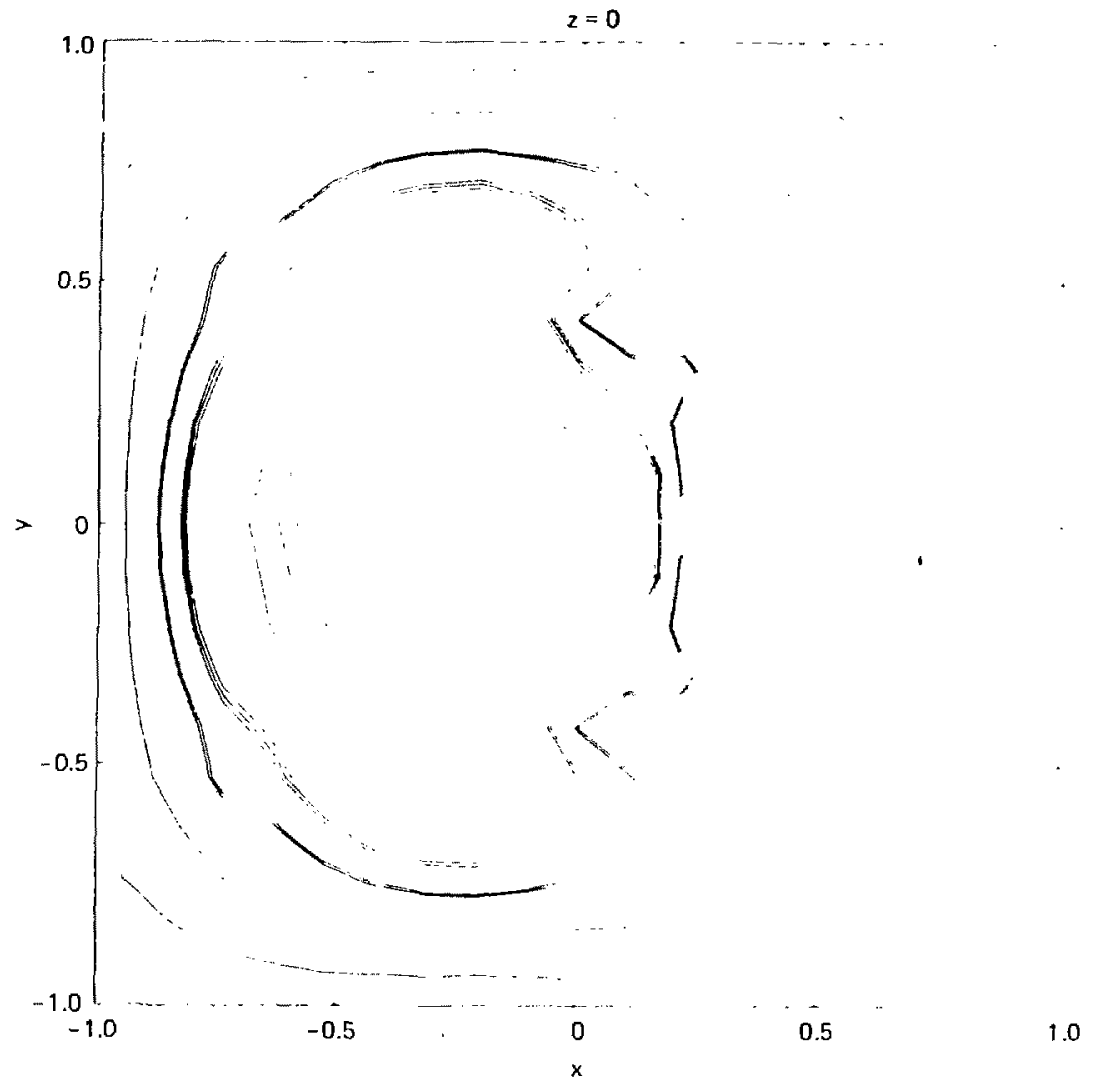

Figure VI.9 Isoplots of density at $t=9.4$ for Case 2. Fote that the
plasma has now been displaced by the effects of the kink. 
$-200-$

22 .

20 .

18

$\stackrel{5}{0}^{\circ} 16$.

14.

12

10

$8+$

0

5

10

15

25

30

35

Time

Figure VI.10 Plot of current (or $\chi$ ) versus time for Case 2. 


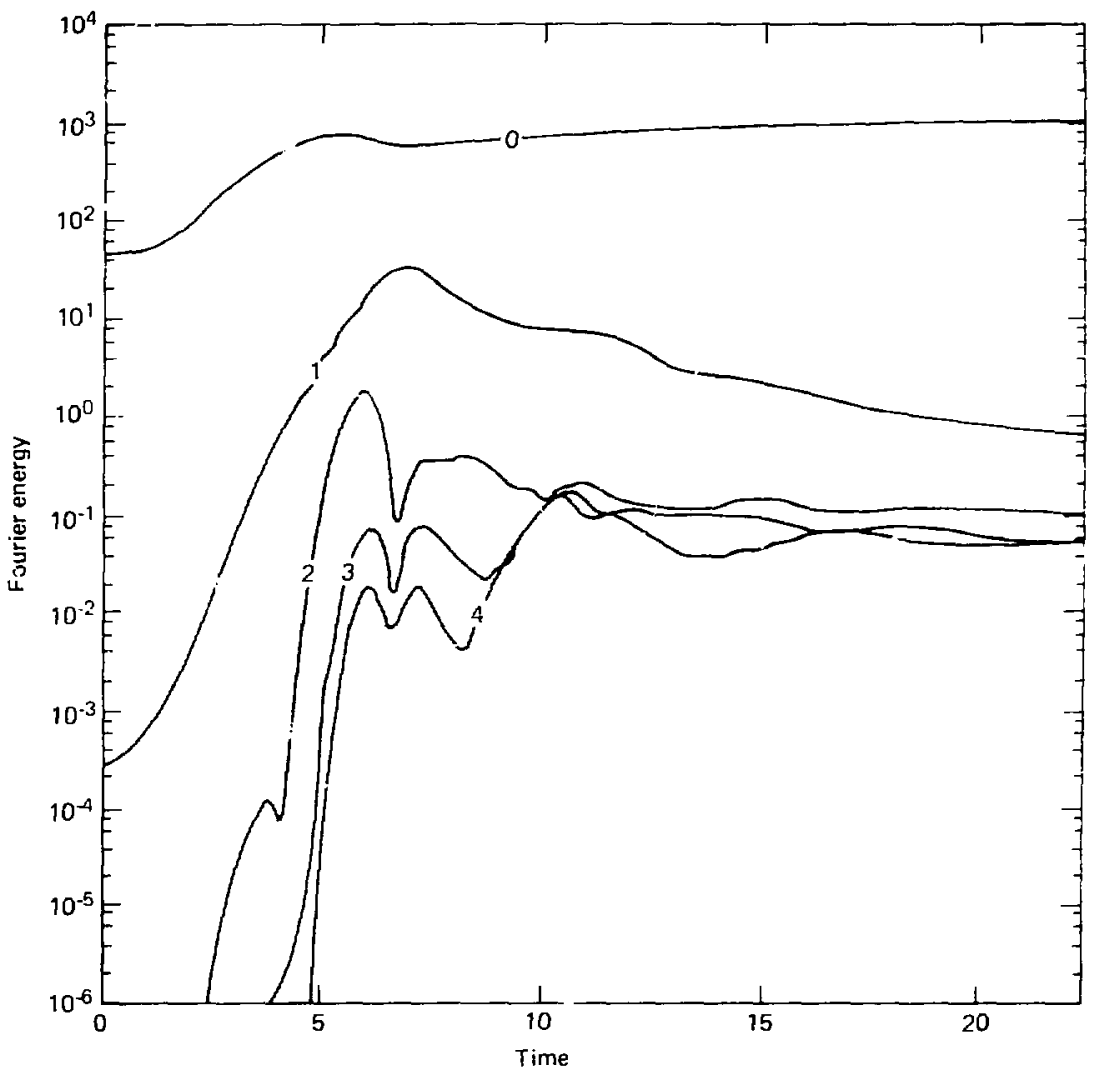

Figure VI.1la Plot of Fourier mode energies versus time for Case 2. 


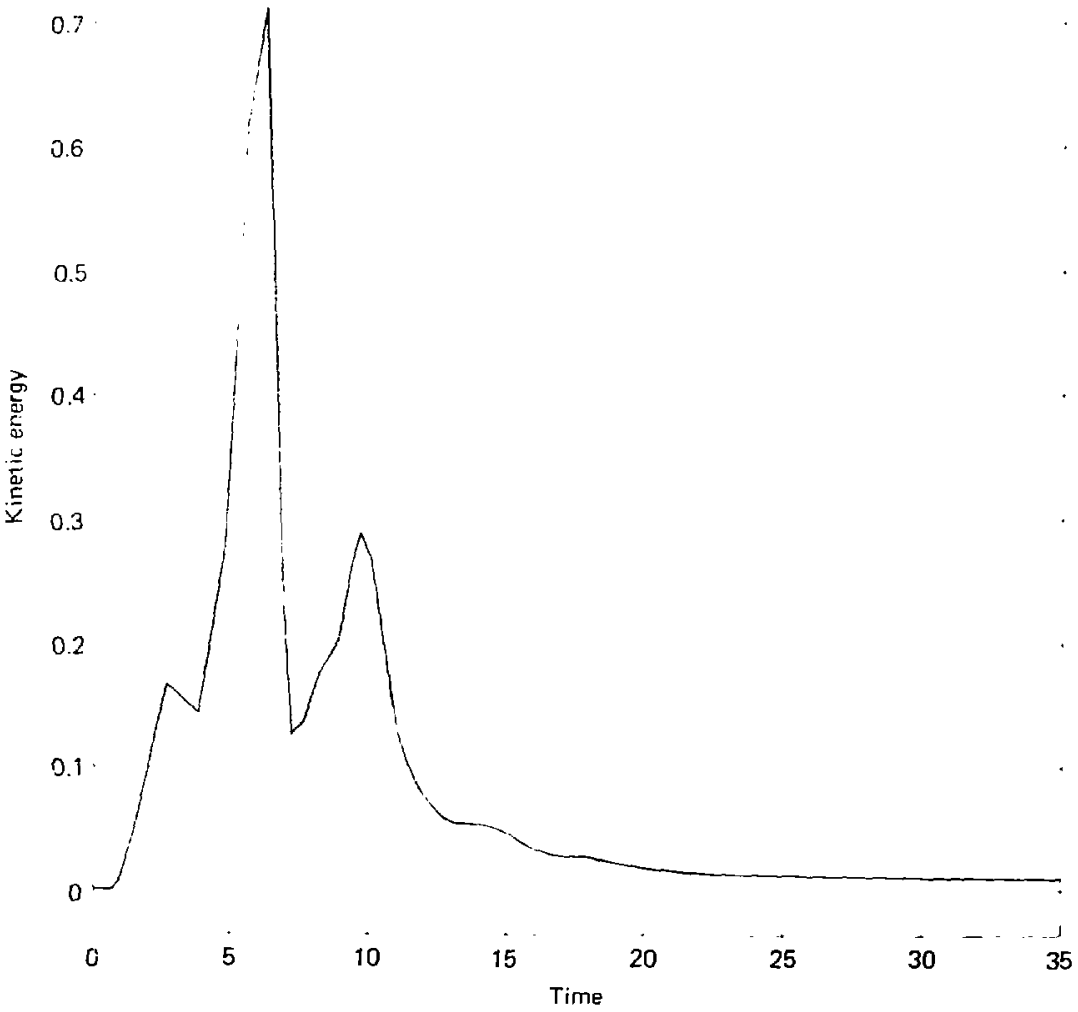

Figure VI.11b Plot of kinetic energy versus time for Case 2. This is plotted on a linear scale to better compare with the results of Sykes and "lesson. 45 
$1.0 \mathrm{r}$

0.8

0.6 .

0.4 .

$\infty^{N^{3}} 0.2 \cdot$

0 .

-0.2 .

-0.4 .

-0.6 .

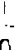

Figure VI.12 Plot of $<\mathrm{B}_{z} \mathrm{w}_{\text {wall }}$ versus time for Case 2 . Note the rapid drop in $\left\langle\mathrm{B}_{z}\right\rangle_{\text {wall }}$ at $\mathrm{t}=6$. Ihts corresponds to the jump in kinetic energy showing in Figure VI.1Ib. $<\mathrm{B}_{\mathrm{Z}}{ }^{2}$ wall then rizes rapidly until another instability (See VI.1lb) causes a flattening of the slope. 


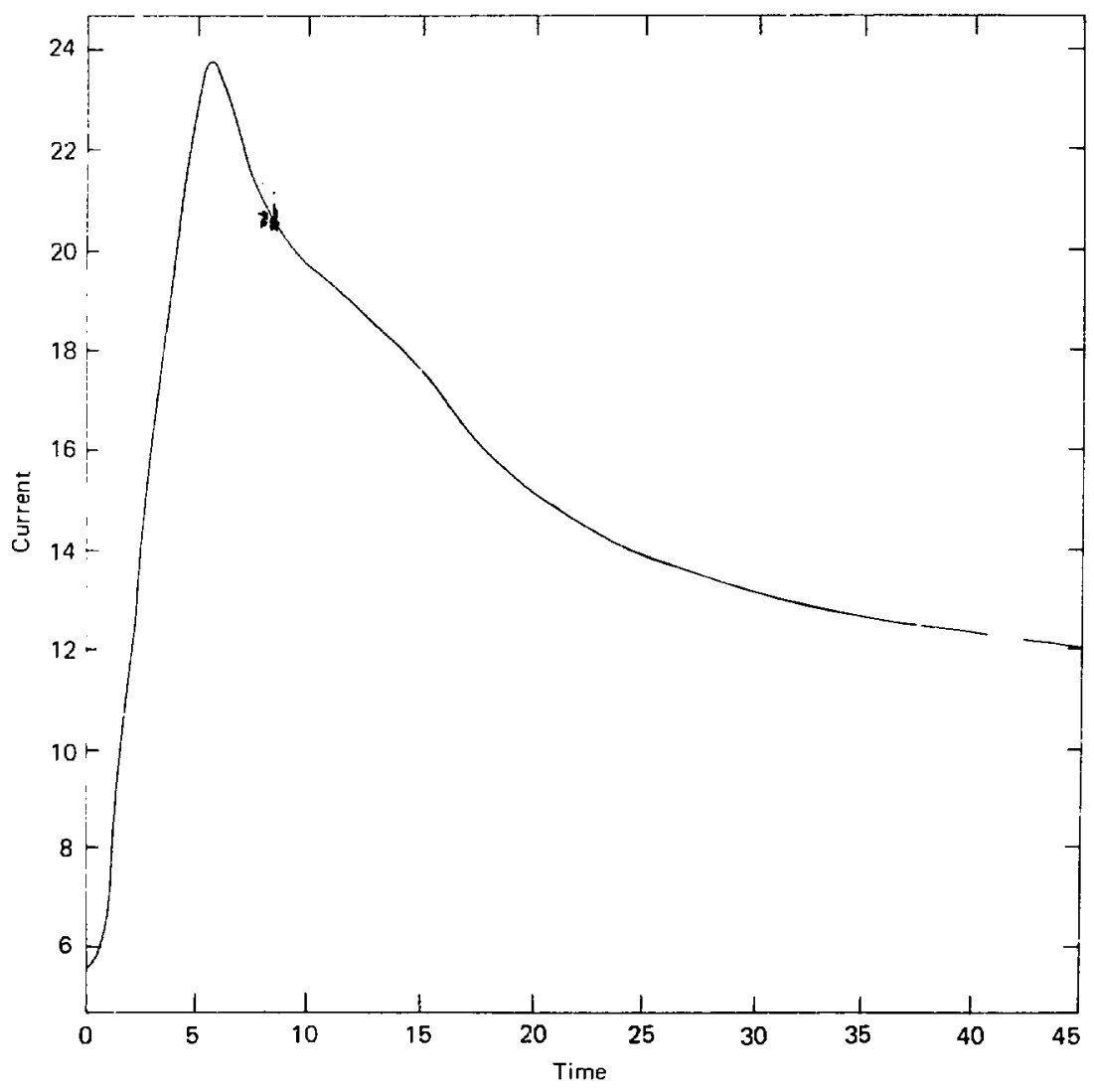

Figure Vi.13a Plot of current (or $x$ ) versus time for Case 3. The current dips tirst at $t=11$ due to a $1 / 1$ mode. This is followed by a sharper drop at $t=16$. 


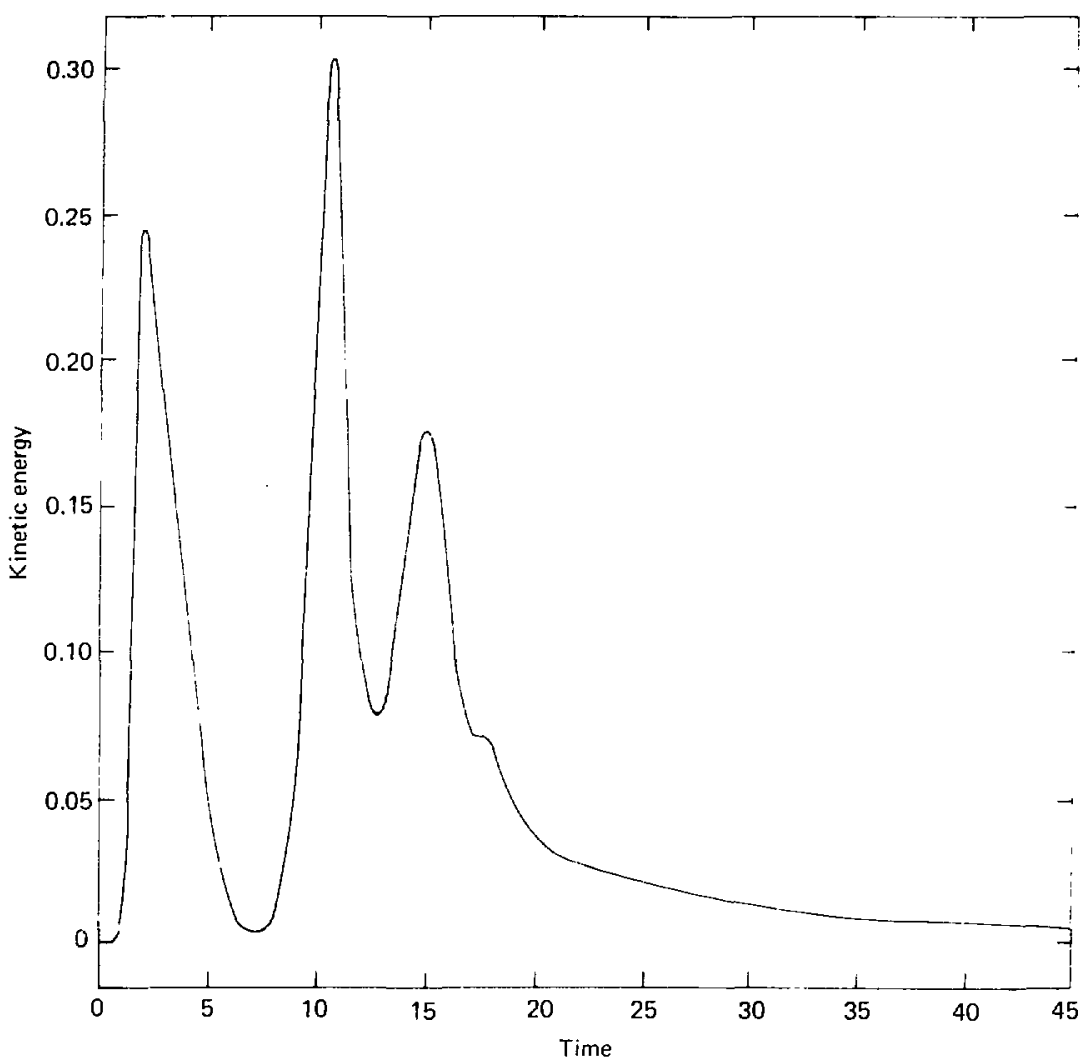

Figure VI.13b lhe kinetic energy for Case 3 plocted versus time. Yote the peaks at $t=11$ and 16 . 


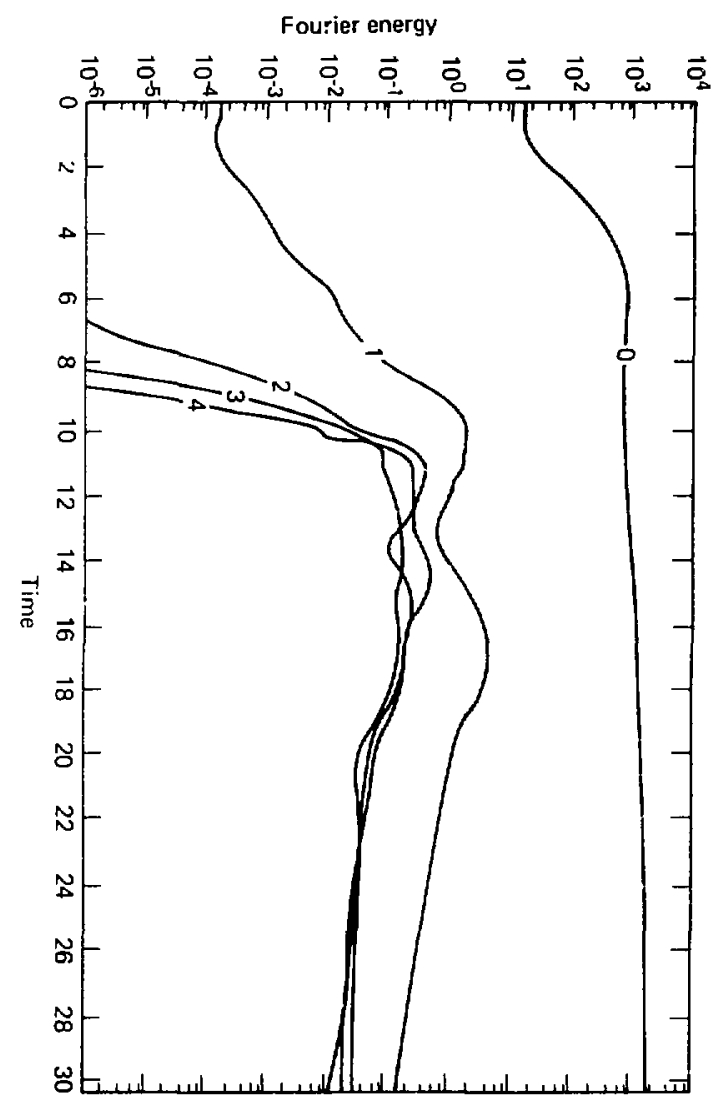

Figure VI.13c The Fourier mode energy versus time for Case 3. 


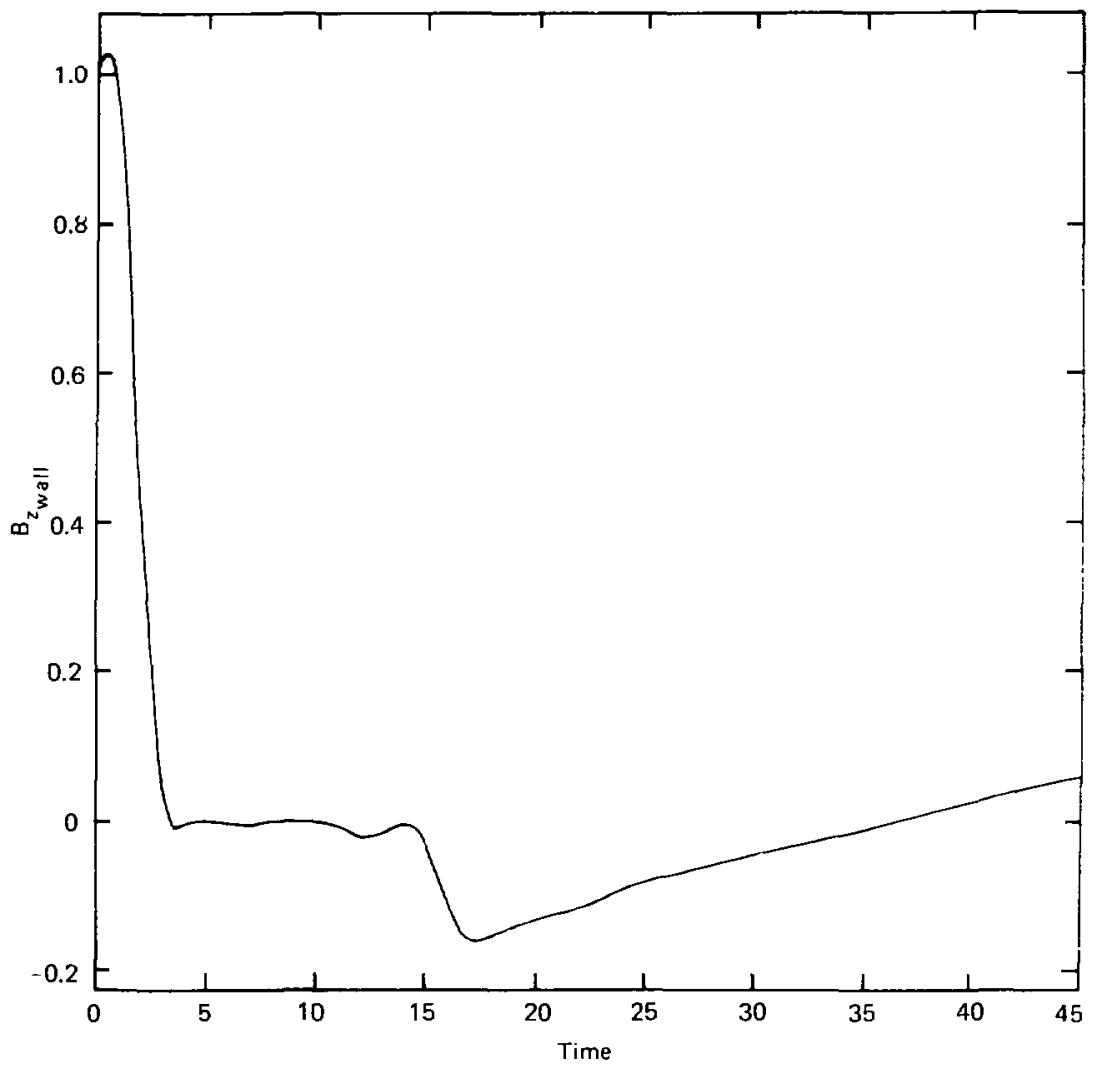

Figure VI.14 Plots of $\angle \mathrm{B}_{\mathrm{z}}$ 'wall versus time. Once again the field reversal corresponds with the peaks in velocity. 


\section{CHAPIER VII}

SUMLARY AND CONCLUSIONS

\section{L S Summary}

The development of the computer program IMP, which craces the evolution of a plasma immersed within a magnetic field, has been detailed. In particular, the physical model used is the set of elght single fluid resistive Mrid equations. These consist of a set of eight coupled, nonlinear partial differei: ial equatio's that consistently describe the interaction of an iorfzed gas with the magnetic fielu. No assumptions have been made to remove the fast tfmescales (e.g., low 5 or Tokamak ordering of the equations $2 b, 50-52$ ). It:e equat ions have been expressed in orthogonal curvilinear coordinates to allow for generality in the problems to wilich the code may be addressed. The equations have been solved rumericaily on a variably spaced Eulerian finlte difference mesh. The equations are advanced temporally by an adaption of the Douglas-Cunn ${ }^{5}$ algorithm for allernating direction implicit differencing. Ihis has avoided the limitations in timestep size that are imposed on explicit techniques. The full set of eight equations has been advanced simultaneously, thus aroiding synchronization errors that can occur when the equations are split for solution.

While the continuity and magnetic flux equations have been treated in full conservation form, the momentum and energy equations have been solved in nonconservation form. This formulation has given enhanced numeric stability by eliminating errors that are introduced by the assumption that $\nabla \cdot B$ is zero on the finite difterence mesh. ${ }^{49}$ This has also allowed the sinulaticii of low B plasmas as the pressure (by the assumption of an ideal gas equation of state), rather than the total energy density, is an independent vartable. 
The boundary conditions that have been per...'ted are quite general in torm (Dirichlet, Neumann, and periodic are alluwed). The conservat lon buundary condition based on the physical properties of the wall ${ }^{54}$ has proven tc provide excellent results.

The techniques tor alscretizing the various terms in the ItW equations have been described. The Newton-Kaphson iteration procerure, which also provides a seconci order iccurate linearifation of the nonlinear algebraic equations that result from the discretization of the partial differential equations, has beer. tully desczibed.

IMP was applied to a number of problems in varying geometries of interest to magnetic fusion researchers. Ideal and resist Ive kink instabilities were treated in Cartesian coordinates. After specifying an inftial analytic equllivrium, an arbitrary--thougn carefuliy choset-- velocity ifelt perturbation led to the emergance of the $M / 2$ ldeal kink instability. Tnis agreed both in form and in growth rate with previous work. ${ }^{y, 5}$ sext, linear eigentunction, generated by the 1 inear $30 \mathrm{HD}$ code of Bateman, 9 was injected it.to the equilibrium plasma. It began immediate exponential growth contirming that an eigenfunction of the linear code was indee 1 extremely : lose to an eigenfunction of the nonlinear code. L'he addition of resistivity was shown to enhance the ideal growth rates without changing appreciabıy the form of the eigenfunctions. A simulation which extended for 90 Alfven transits displarzd a new plasma state that was characterized by an extrempsy ilowly growing $3 / 1$ mode. A final simulation was performed which allowed the finite resistivity to affect the initial equilibrium. While the velocity and density showed marked response to the magnetic diftusion and ohmic heating, the $1 / 1$ growth rate was unchanged. 
Next, a cylindrical coordinate system was used to examine the effects of curvature on the kink growth rates. An initlal numeric equilibrium that corresponded to the first Cartesian simulation was perturbed by elgenfunctions generated by the 1 inear Ioroidal code of Hicks and Wooten. 71 once again, immediate exponential growth of the k1nk mode was observed, and growth rates were found to be within $1 \%$ of the linear values. A resistive stmulation of a $1 / 1$ mode in an aspect ratio 3 torus was allowed to progress for more than 1300 Alfven transit times. This was a very slowly growirg modr - nd we did not attempt to resolve its growth rate. Rather, we followed its evolution until a new axisymmetric equilibrium that possessed a 1/0 flow field near the outer radius appeared. Timesteps were attained that corresponded to Courant numbers in excess of 60 .

Finally, the spontaneous reversal of the magnetic field about a high pinch ratio plasma was demonstrated. This proved to be the most difficult simulation for a number of rea, ins. In the case of an initial high pinch ratio plasma, the singular layer is quite close to the boundary. In order to minimize the number of mesh points while maintaining reasonable resolution near tne wall, a variable mesh spacing was used $\left(\Delta x_{\text {wall }} / \Delta x_{\text {center }} \approx 10\right)$. The plasma displayed a late time reversal of $B_{z}$ brought about by a $1 / 1$ resistive kink. This is in agreement with the results of Pritchett. 74,75

The most challenging problems that were simulated were the initially low jinch ratio plasmas that were subjected to time varying external electric fields. In these cases, the plasma was pinched or compressed by the magnetic Eields. The compression led to numeric difficulties that had to be resolved 
by the inclusion of a Von Newmann ${ }^{56}$ artificial viscosity in the momentum and energy equations. Once the time dependant electric field and the artificial viscostty were included, sood qualitative agreement with Sykes and Wesson, ${ }^{45}$ and Gowers, et. al ${ }^{46}$ was obtained.

VII. $\quad$ Conclusions

For the internal kink modelled in a Cartesian coordinate system, it has been shown that IMP can accurately determine the linear growth rates and can trace the nonlinear evolution of the plasma through the saturation of the $1 / I$ mode. With the inclusion of resistivity in the model, the presence of a new plasma state that contains only a slowly growing $3 / 1$ mode is detected. The variation of magnetic Reynolds number S simulations showed that Increased resistivity enhanced the growth rate of the $1 / 1$ mode. This was in agreement with the theory of Coppi, et. a1. 7

The simulations performed in a torus in cylindrical geometry have demonstrated the versatilfy of the computational model in IMP. The lowered growth lates and final velocities attained are due to the stabilizing effects of the curvature which causes the plasma column into closer proximity with the outer wall. IMp was able to trace the nonlinear evolution of the $1 / 2$ mode in only 280 cycles and on a much coarser mesh than was used in the linear code. Finally, the existance of a final axisymmetric state wich finite-though non-growing--flow fields shows the necessity for having fully nonlinear models which can trace a plasma's state through long periods of time.

The reversed field pinch (RFP) calculations have led to independent confirmation of the simulations of sykes and Wesson, ${ }^{45}$ and Gowers, et. a1. ${ }^{46}$ The main difference in our models is that IMP uses a scalar resistivity which does not cause as much peaking of the current as does the tensor model of 
sykes and wesson. However, the conclusion that the nonlinear $1 / 1$ resistive kink, (oupled with higher $n$ kink modes $(1 / 2$ and $1 / 4)$, is the driving mechanism that leads to the spontaneous field reversal is born out in our calculation.

The use of variable timestep size controls and Newton-Raphson iterations Lo a convergence criterion have proven to be extremely valuable to the success w tnis ode. Once again, the inclusion of an artificial viscosicy has also becn slown to be required when any compression is occuring. Finally, the zencral mudel in IMP has been shown to be a useful cool--although one whose use must be carefully considered--in the study of magnetized plasmas.

VII.3 Fukure Hork

Much work lies ahead in the developemnt of realistic three-dimensional codes. In our model, only the lirst non-ldeal transport term, resistivity has been included. The next important term to include is thermal conductivity. Following this, is model to describe the energy losses due to radiation must be included. ${ }^{45,69}$ Also, one must consider the actual transport of radiation throughout the plasma if the plasma becomes optically thick. 76

$\Lambda$ treatment of concentration points of coordinate systems inust be included to allow the simulation of circular devices. Also, a better understanding of the cifects of mixed derivatives on the time centering and statility of numeric techniques must be achieved.

While much is still to be done to :mprove the physics model cantained in IMP, the implementation of the algorithm must also be considered. 
The next generation of IMP, CRIMP (CRAY IMP), will be designed to run on the CRAY-1 computer. While the current IMP can execute on the CRAY, it is grossly inefficient for this single level memory, vector processor.

In this section we will be repeatedly referring to vectors and vectorizability. Therefore, we must have a working definition of a vector and what is veciorizable. The term vector on a computer refers to an array of $\mathrm{n}$ elements separated in core by some constant increment $\mathrm{m}$. A vectorizable sequence of code is one in which the same operations are performed on all the elements of a vector. An example of this 1s

$$
\begin{aligned}
\text { DO } 10 L & =1,1000 \\
A(I) & =B(I) * C(I)+D(I+1) * C(I+1)
\end{aligned}
$$

10 CONTINUE

Here A, B, C, and D are vectors, and we are performing the same operations on all 1000 elements. The following loop is not vectorizable:

DO $20 I=2,1000$

$$
A(I)=A(1-1)+B(I) * C(1)
$$

20 CONTINUE

The reason that this loop is not vectorizable is because the computation of $A(I)$ requires the value of $A(I-1)$. This is only known after the previous computation. The vector processor works by starting a string of up to 64 numbers (on the $\mathrm{CRAY}$ ) through a vector funtional unit. The first answer comes out after the same number of clock cycles as it would from a scalar unit with the same function. After the first answer comes out, each succeeding answer 
is returned at the rate of 1 per clock period. In the case of loop 20, the input strean of numbers for $A$ is unknown.

As was explained in Chapter III, the basic nonlfnear algortthm solved is as follows. First we bring a cow of our mesh into SCM/SSM. * Next we repeatedly use a block tridiagonal solver to advance as many Newton-Raphson iterations along the row as are required to achieve a convergence crfterion. We then store l.he results back in LCM** and repeat the procedure on the next row. First (i) all, on the CRAY we do not need to move the information around in memory. Next, the solution of the block-tridiogonal matrix requires the inverston of smaller \& hy \& submatrices at each grid point. This is accomplished via Ll decomposition. The LU decomposition is inherently non-vectorizable since we must solve i recursion relation. The way to convert this to a vectorizable problem is to iterate $n$ rows of our mesh simuitaneous $1 \mathrm{y}$, where $\mathrm{n}$ is a number in the range 1 to 64 . This vectorizes the block-tridiagonal solver and also vectorizes almost all calculations required to generate the elements of the matrix.

One other change that could lead to a great savings in time is to change our basic iteration technique. The Newton-Raphson iteration technique converges at an $\varepsilon^{2}$ rate, ${ }^{61}$ but it forces us to invert the blocktridiagonal system. The Cord method uses the value of tine Jacobian at an older iteration to approximate the correct Jacobian and on 1 y requires a matrix multiplication to give us our next set of iterates. We can, therefcre,

* Small Core or Semiconductor Memory

** Large Core Memóry 
use the full tridiagonal solver to get a Jacobian, then use this Jacoblan for a number of iterations, and, perhaps, even for a number of timesteps, until the iterations fall to converge. We then update the Jacobian via the full block-tridiagonal solver. This new technique could lead to speed-ups of an nrder of magnitude or more by the elimination of most of the Jaronian inversion. 


\section{ACKNOWLEDGHENTS}

blit: work ranla never have been completed without the undying love,

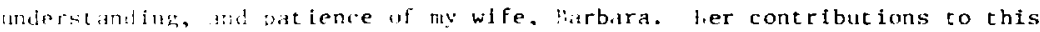
work anmer be werstatrd.

: wi to tank professur John killeen for origlnally suggesting thls

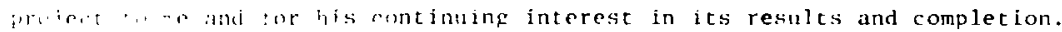

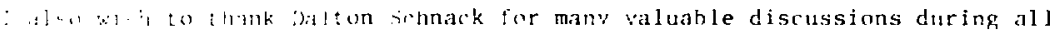

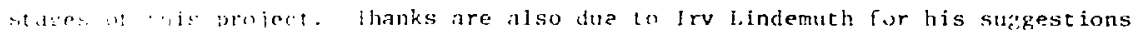

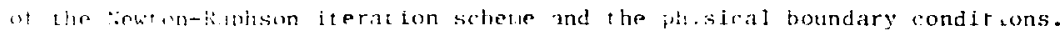

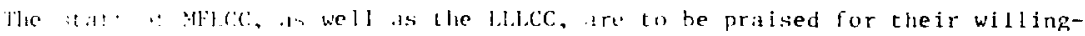

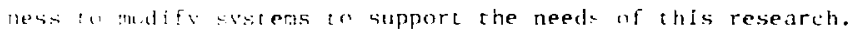

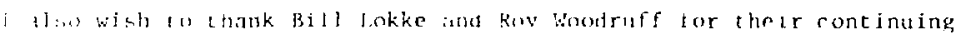
Guport. . Susin Finn, Wu labored to convert mo seribbles into this manbserint, mu to Arns Aartuli, who laid the proundwork for the preparation af Lise molitude of tigures contained in this thesis, I uffer mu neartelt thanks. To the many others tho aided in the suressful rompletion of this pro: at. lhank you all 
REFERENCES

1. 1). J. Rose and M. C. Clark, Pasmis and Controlled Fision, MIT Press, Cambridge, Mass. $(19(9)$ ).

2. J. D. Lawsun, Proc. koy. Suc. (London) B?0, : (1957).

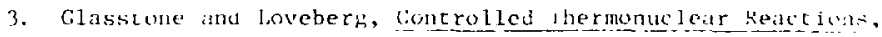
van Nostrand Reinhold lo. New York (1960).

4. I) h. Peaceman and II. H. Kathford, Jr, Stat Joumal 3, $\therefore(14$,$) .$

5. J. Douglas and J. Gumn, Sumer. Yath. 6 Pj $228-45,(12641$.

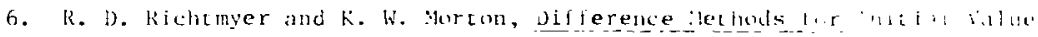
problems, second Fation, Intersience, New gurk (196, .

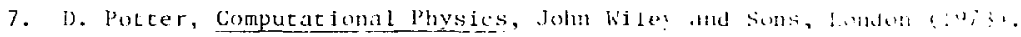

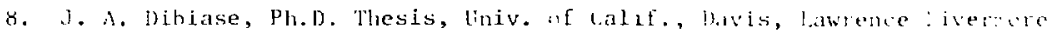
Laboracory keport UCRL-S15y] (1974).

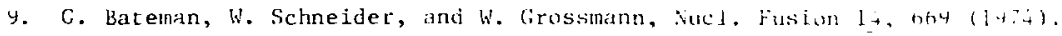

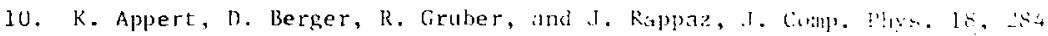
$(1975)$.

11. J. 1. Johnson, M. S. Chance, J. H. Greene, R. C. Grima, ․ kerner, in Proc. 7tn lurop. Con [., Lausame (1975). 
1.2. U. Berger, R. Gruber, and $r$. Tryon, Computer Phys. Comm. 11,313 (I976).

13. R. C. Cirimm, J. M. Creene, and J. L. Johnson in Methods in ComputaLiond l'hysics, Vol. 16, J. killeen (Ed.), Arademic Press, New York $(1976)$.

14. A. M. Todi1, Y. S. Chance, J. M. Creene, P. C. Crimm, J. L. Johnsor, and Manickith, Mliys. Rev. Letters 38, 826 (1977).

15. 1. H. Bernitein, I., A. Frieman, M. D. Kruskal, and R. M. Kulsrud, Proc. loy. Soc. (london) 1244,17 (1958).

16. D. 1). Schnack and J. Killeen, Nucl. Fusion 19, (1979), in press.

17. 1). D. Scnuack and I. Killeen, in Theoretical and Computational plasma l'hysiss, lalı, Vienna, 337 (1978).

13. A. A. Vedenov, in Reviews of Plasna Physics, Vol. 1, M. A. Leontovich (Ed.), Consultants Bureau, New York (1965).

19. K. V. Roberts, F. Hertweck, and 5. J. Roberts, Thetatron: A TwoDimensional Magnetrohydrodynamic Computer Program, Culham Laboratory Report CLM-R-29 (1962).

20. K. V. Roberts and N. O. Weiss, Math. Comp. 20, 272 (1966).

21. N. 0. Weiss, Proc. Roy. Soc. A293 (London), 232 (1966).

22. D. Duchs, Phys, Fluids 11 , 2010 (1968). 
23. J. R. Freeman and F. O. Lane, APS Topical Conf. Numerical Simulation ot Plasma, Los Alamos, Los Alamos Scientific Laboratory Report Li-3990, Paper Ci (1968).

24. N. J. Peacock, P. D. Wilcock, R. J. Speer, and P. D. Morgan, Third IAEA Conf. Plasma Phys. and Controlled Nucl. Fusion Res., Novosibirsk, Paper $\mathrm{CN}-24 / \mathrm{G}-4(1968)$.

25. K. V. Roberts and D. E. Potter, in Methods in Computational l'hysics, Vol. 9, B. Alder, S. Fernback and M. Rotenberg (Eds.), Academic Press, New York (1.970).

26. A. Lapidus, J. Comp. Phy's. 2 , 154-1/7 (196/).

27. A. Sykes and J. A. Wesson, Nuc1. Fusion 14, 645 (1974).

28. R. White, D. Monticello, M. N. Rosenbluth, H. Strauss, and B. B. Kadamtsev, Plasma Physics and Controlled Nucl. Fusior, Tokyo, IALA-CN-33/A13-3 (1973).

29. R. White, U. Montice110, M. N. Rosenbluth, and B. Waddel1, Phys. Flu1ds $20,800(197 /)$.

30. R. L. Morse, in Methods in Computatzonal Physics, Vol. 9, B. Alder, S. Fernbach, and M. Rotenberg (Eds.), Acedemic Press, New York (1970).

31. F. Hertweck and W. Schneider, Proc. Second. Europ. Conf. on Controlled Fusion and Plasma Phys., Stockholm (1967). 
3L. D. V. Anderson, J. Comp Phys. 17, 246 (1975;)

33. S. Jardin, J. Comp. Phys. 29, 101 (1978).

34. I. Lindemuth and J. Killeen, J. Comp. Phys. 13, 18: (1973).

33. I. Lindemuth and T. Jarboe, in Proc. of the Annual controlled Fusion Theory Yeeting, Paper 18, San Diego, CA (197/).

36. H. C. Lui and C. K. Chu, Phys. Fluids $18,12 / 7$ (1975).

37. H. C. Lui and C. K. Chu, Phys. Fluids 19, 19/4 (1976).

38. D. Schnach and J. Killeen, J, Comp. Phys. 35, 110 (1980).

39. J. Killeen, Information Processing 71, North-Holland Publishing Co., $1191(19 / 2)$.

40. J. Brackbil1, in Methods in Computational Physics, Vol. 16, J. Killeen (Ed.), Academic Press, New York (19/6).

41. J. Wooten, H. R. Hicks, G. Bateman, and R. A. Doxy, Oak Ridge National Laboratory Report OKNL-TM-4784 (1974).

42. F. L. Cochran and P. C. Liewar, in Proc. of the Eightn Conf. on Numer. Simulation of Plasmas, Paper PB-3, lawrence Livermore Laboratory Report CONF-180614 (19/8).

43. P. L. Prltchert, C. C. Wu, and J. M. Dawson, Phys. Fluids 21, 1543 (1978).

44. A. Sykes and J. A. Wesson, Phys. Rev. Letters 37, $140(19 / 6)$. 
45. A Sykes and J. A. Wesson, 8th Europ. Conf. on Controlled Fusion and Plasma Physics, Prague (1977).

46. S. von Goeler, W. Stodiek, and N. Santhoff, Phys. Rev. Letters, 33, $1201(1974)$.

47. J. P. Christiansen and K. V. Roberts, Nucl. Fusion 18 2, 181 (1978).

48. C. W. Hirt, A. A. Amsden, and J. L. Cook, J. Comp. Phys. 14 (1974).

49. J. U. Brackbill and D. C. Bames, J. Comp. Phys. 35, 421, (1980).

50. H. R. Strauss, Phys. Fluids 19, 134 (1976).

51. S. C. Jardin and J. L. Johnson, in Proc. of the Eighth Conf. on Numerical Simulation of Plasma, Paper OB-2, Monterey, CA, Labrence Livermore Laboratory Report CONF-780614 (1978).

52. H. R. Hicks, B. Carreras, J. A. Holmes, D. K. Lee, S. J. Lunch, anri B. V. Waddell, in Proc. of the Eighth Conf. on Numerical simulation Plasma, Paper PA-7, Monterey, CA, Lawrence Livermore Laborator: Report CONF-780614 (1978).

53. A. C. Hem and M. L. Griss, REDUCE 2, USERS MANUAL, Ind Edition, UCP-19 (1975).

54. I. R. Lindemuth, J. Comp. Phys. 18, 119 (1975).

55. H, R. Hicks, private communication.

56. J. Von Neumann and R. D. Richtmayer, J. App. Phys. 21, 232 (1950). 
57. At. P. Furth, J. Killeen, and M. N. Rosenbluth, Phys. Fluids 6,459 (1963).

58. J. Brackbill, ;n Annual Controlled rusion Therry Conf., Paper B-29, (iat 1 inburg, Tenn. (19/8).

5y. J. Brackbill and D. C. Barnes, to be pubiished in J. Comp. Ph; s.

hi). P. Y. Morse and H. Feshbach, Methods of theoretical Physics, Vol. 1, 1. Lf, Mr (iraw-Hill, New York (1951).

b1. H. R. Pennington, Introductory Computer Methods and Numerical Analysis, Macmillin, New York (1965).

6.) A. C. Hindmarsh, Banded Linear Systems with Pivoting, Lawrence livermore Laboratory Report, UCLD-30060, Rev. 1 (1976).

63. I. R. l.indemuth, Ph. P Thesis, Univ, of Calif., Davis, Lawrence Livermore Laboratory Report UCRL-51103 (1971).

64. J. Killeen and K. D. Marx, in Methods in Computational Physics, Vol. Y, B. Alder, 5. Fernbach, and M. Rotenberg (Eds.), Academic Press, New York $(19 / 0)$.

65. L. Spitzer, Jr., in Physics of Fully Ionized Gases Second Edition, Interscience, New York (1962).

66. W. R. Briley and H. McDorald, J. Comp. Phys. 24, 37? (1977).

67. A. C. Hindmarsh, L. J. Sloane, K. Fong, and G. H. Rodrigue, DEC/SOL: Solution of Dense Systems of Linear Algebraic Equations, Lawrence Livermore Laboratory Report UCID-3013/ (1976). 
68. P. J. Roach, Computational Fluld Dynamics, Hermosa Publishers, Albuquerque, NM (1972).

69. I. R. Lindemuth, The ANIMAL Code, Lawrence Livermore Laboratory Report UCRL-52492 (1979).

70. B. Coppi, R. Galvão, R. Pellat, M. Rosenbluth, and P. Rutherford, Fizika Plast.ly, 6 , 961 (1976).

?1. H. R. "icks and J. W. Wooten, Oak Ridge National Laboratory Report ORNL/CSD/TM-3 (1976).

72. E. 1. Butt, C. W. Cowers, A. Mohri, A. A. Newton, D. C. Robinson, A. J. L. Verbage, M. R. C. Watis, Li Yın-An, H. A. B. Bodin, in Proc. of Seventh European Conf. on Controlled Fusion and Plasma Phys., Vol. 1, 39 (1978).

73. J. B. Taylor, in Pulsed High Beta Plasmas, D. E. Evans (Ed.), Pergamor Piess, oxford (1976).

74. P. L. Pritchert, C. C. Wu and J. M. Dawson, in Proc. of Annual Coucrolled Fusion Theory Conf., Paper C16, San Diego, CA (1977).

75. P. L. Pritchert, private communication.

76. Ya. B. Zel'dovich ana Yu P. Raize'. Fhysics of Shock Waves and High Temperature Hydrodynamic Phenomena, Vol. 1, Academic Press, New York, NY (1966). 
APPENIIX $A$

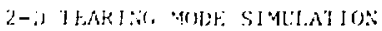

The iwo-ifinensional tearing mode instabllity in a sheet pinch wia;

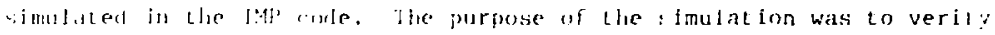

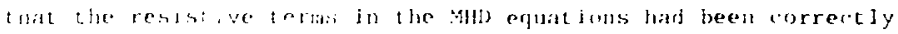
iaburpurated inte thre ende.

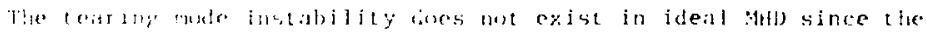

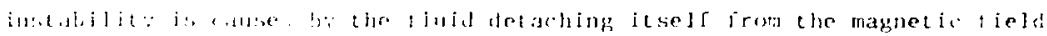

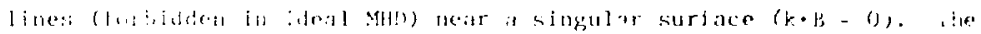
conditions an wert whenever the ficlu is sheared or reversed. The fludi

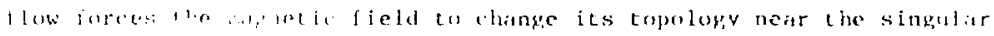
surdace. The : seli fibes tear and reconnect about the singular surface

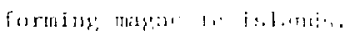

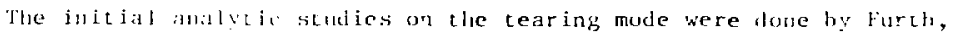
killeen and Robenblath. 7 they gave a growth rate proportional to the manctic kevmolds number $\mathrm{S}^{2 / 5}$ (see chapter II). The gruwth rate was slow compared to ideal MHi timest ales but faster than the resistive diffusion timescale, $a^{-3 / 5}-2 / 5$. These reites were also obtained and extended computationally into the nonlinear regime by schuack.

For the test of IMP, a copy of Schnack's code was cblained and outh codes were started with identical initial conditions. Due to che sharpness of the tearing laver, both codes used identical nonuniform mesh spacing for betcer resolation in this region. This also, then, tested our nonunform zoning c'spability. Figure A.L shows the initial flux contours in both 


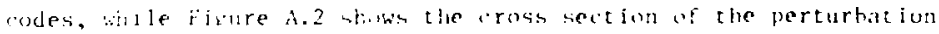

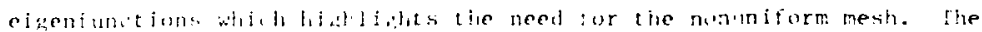

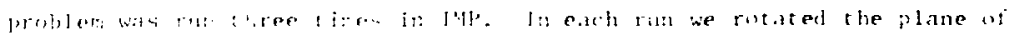

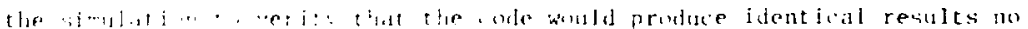

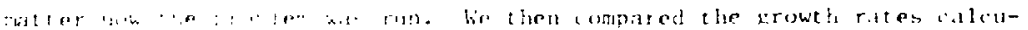

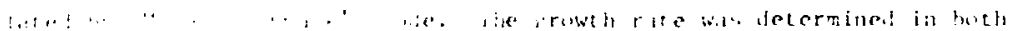

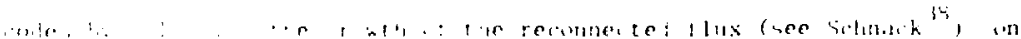

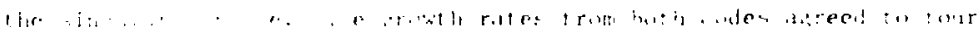

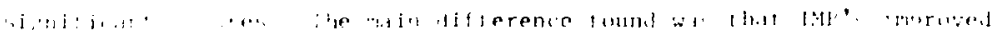

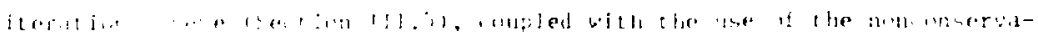

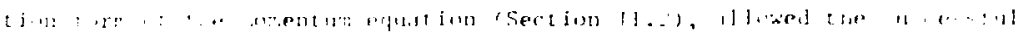

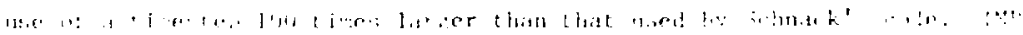

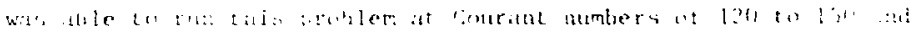

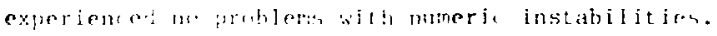

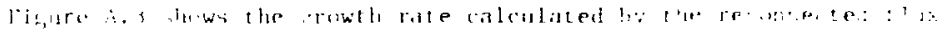

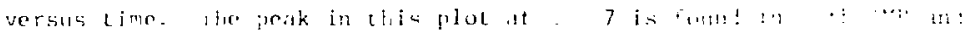

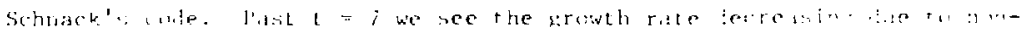

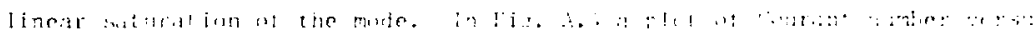

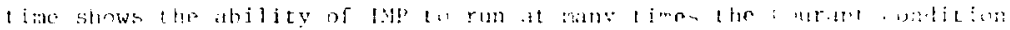

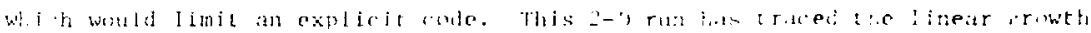
$\because$ the Learing mode and the unset of nonlinear mituration in - t rmatepis-less Lhan 10 minutes of (D) $-76(30)$ time. 


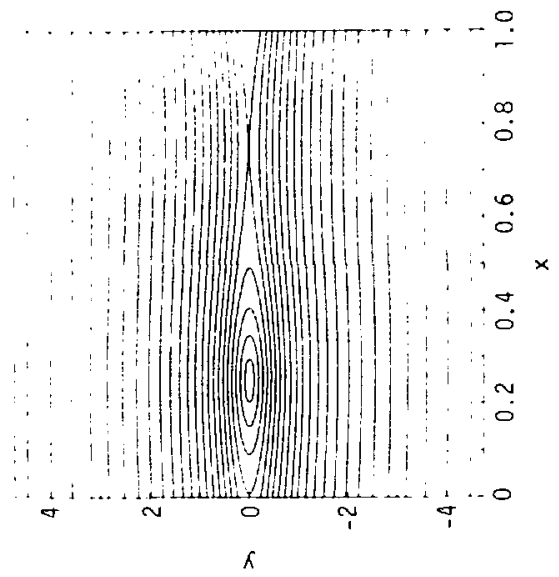

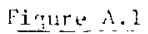

We initial magentic flux contours used in both JilP anc Schnack's corle. 


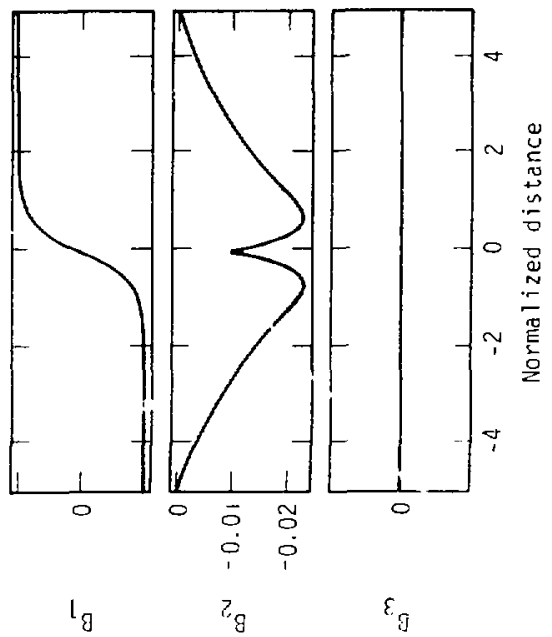

Figure A.2a The perturbation elgenfucntions of magnetic field used in hoth IMP and Schnack' code. Sote the stronily peaked nature of $B_{y}$ as it crosses the origin. 


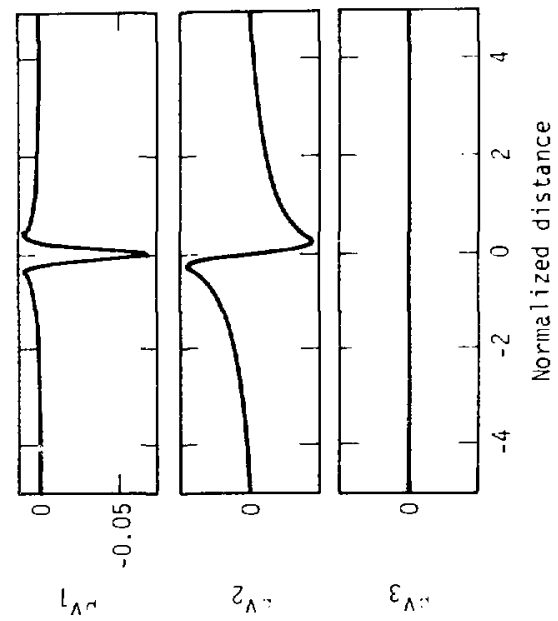

Figure A. used. Once agrain, note the highly localized nature of the perturbations near the axis. 


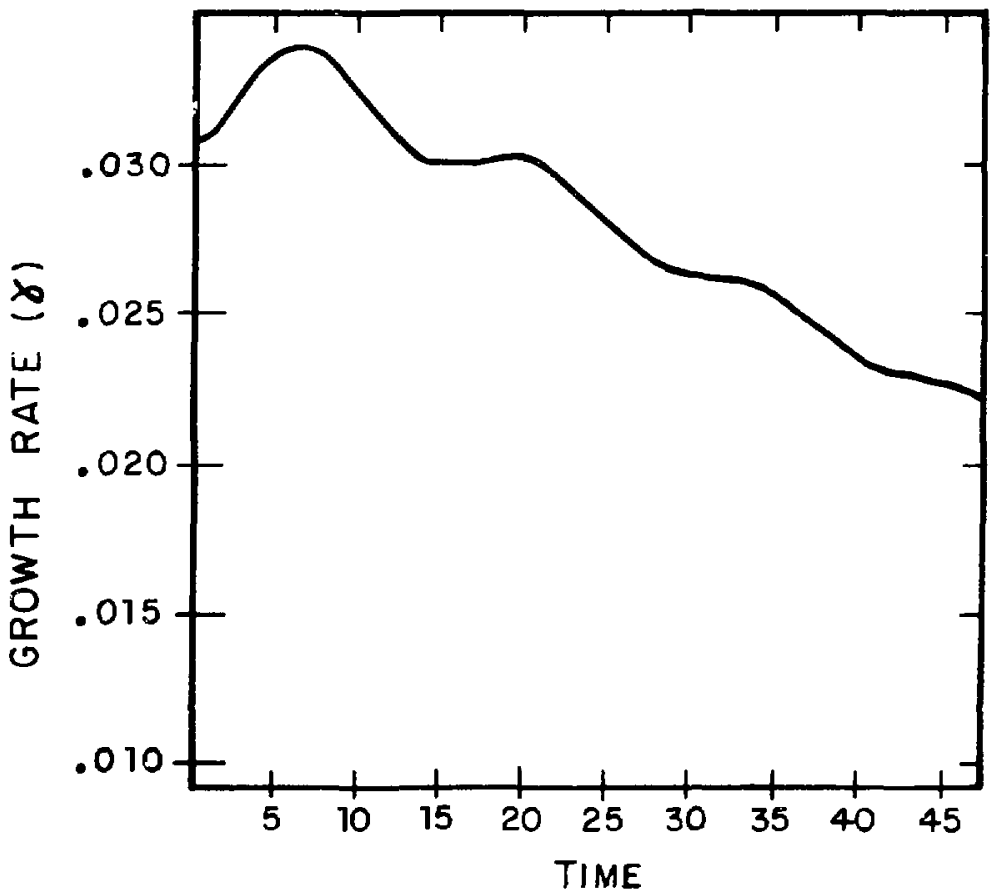

Figure A.3 The growth rate of the tearing mode instability calculated by IMP. The peak at $t-7$ was found in both our model and Schnack's. 


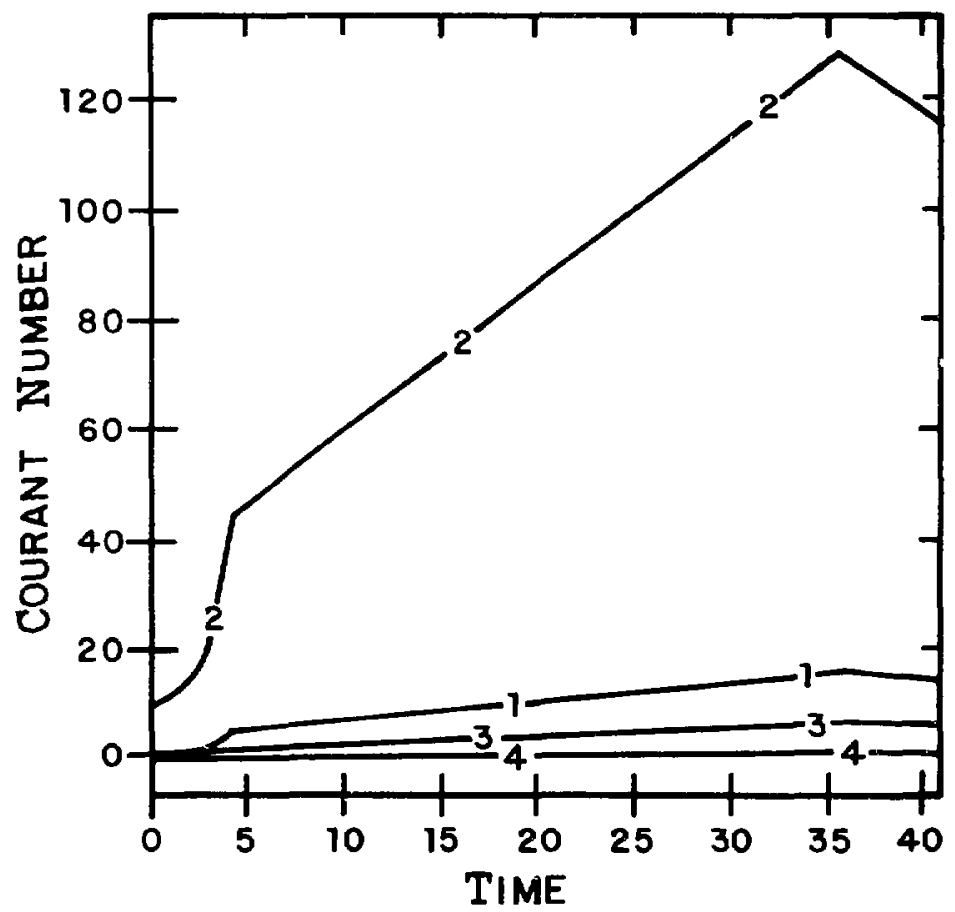

Figure A.4 The Courant numbers are plotted versus time for IMP in the preceeding stmulation. The traces, labeled 1-4, are for the Courant number for 1) $x$ direction hydro, 2) $y$ direction hydro, 3) z direction hydro, and 4) resistive diffusion. 


\section{APPENDIX B}

\section{The REDUCE Generator Code}

The source code used to generate the Jacobian motrix elements via the REOUCE algebraic code system is I isted below.

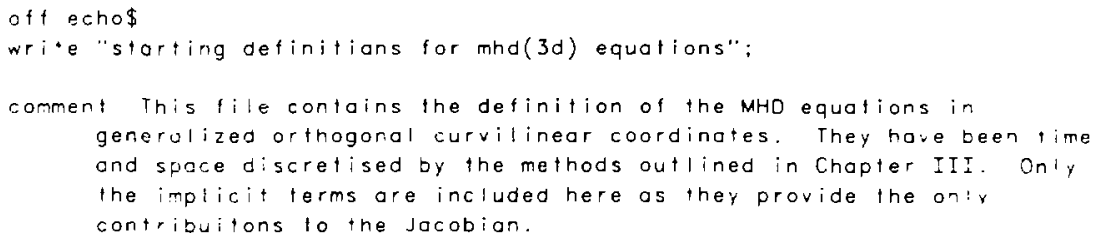




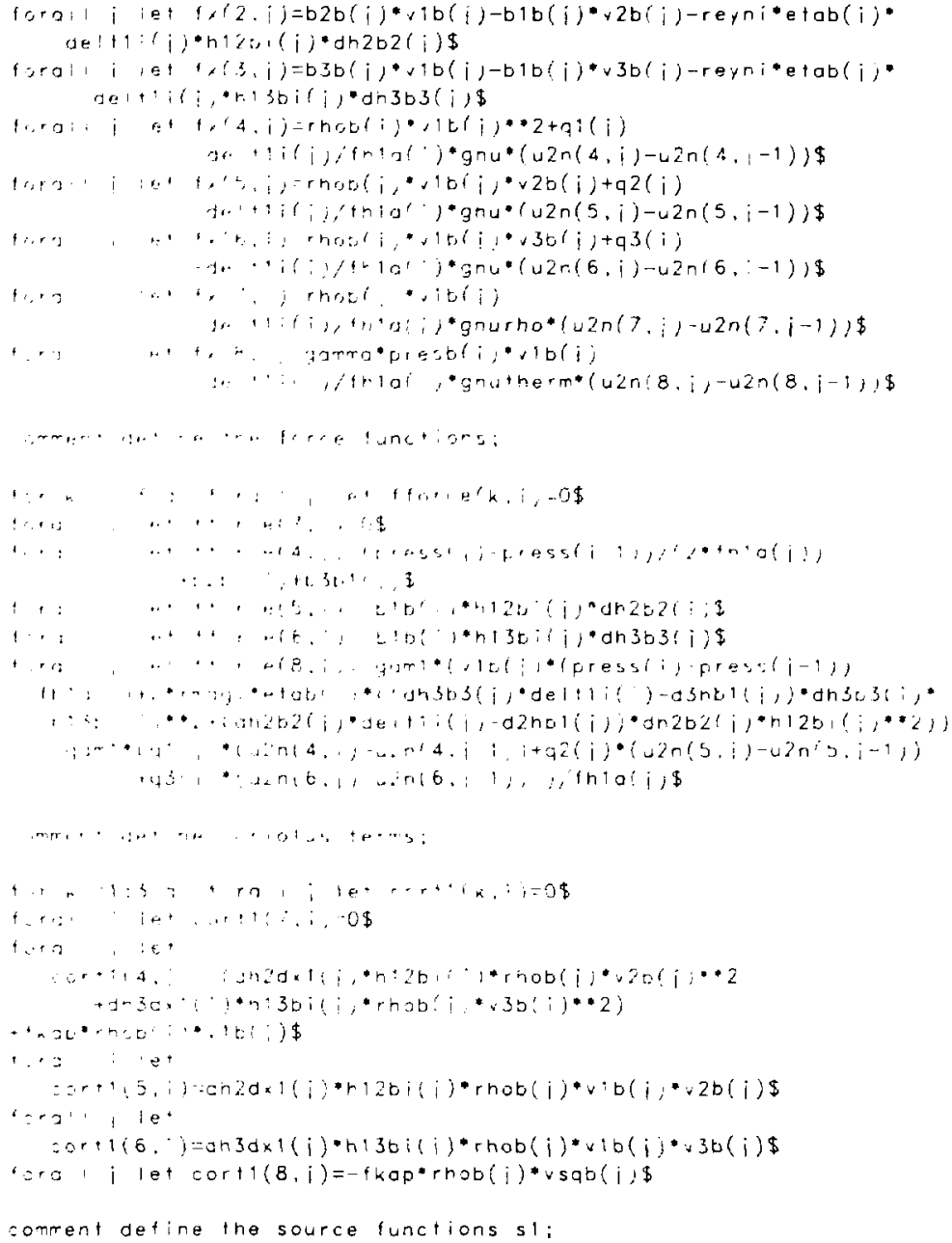


comment define the bar functions to the progrom var-swu;

\begin{tabular}{|c|c|c|c|}
\hline foral & $i$ & $1 \mathrm{et}$ & press $(i)=42 n$ \\
\hline foral: & i & $1 \in \dagger$ & $\mathrm{b} 1 \mathrm{o}(i)=\mathrm{sw}(1, i) \$$ \\
\hline forali & i & $1 e t$ & b2b $(j)=5 w \cup(2, j) \$$ \\
\hline foral & i & $\operatorname{let}$ & b3t $(i j=5 w 4 i 3 . j) \$$ \\
\hline forg: & $i$ & $1 \notin \dagger$ & $\cdot \mid b(i)=\operatorname{sw} u(4, i) \$$ \\
\hline for & i & $1 E 4$ & $2 b(i)=\operatorname{swu}(5 . i) \$$ \\
\hline $\mathrm{fora}$ & i & Iet & $.3 t, j=\sin u(6, i) \$$ \\
\hline foral & i & let & rhot $(\mathrm{i})=\operatorname{sww}(7 . \mathrm{j}) \$$ \\
\hline forol & & $i \omega^{+}$ & $\operatorname{cosec} i)=\operatorname{swu}(8 . j) \$$ \\
\hline
\end{tabular}

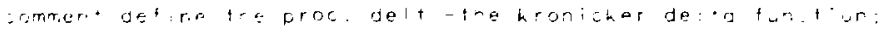

integer arecoluen telti,kit

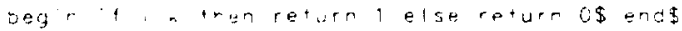

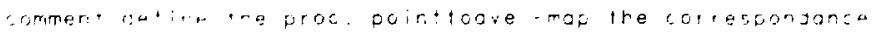

ot r r we r...m. to ar averoge point\$

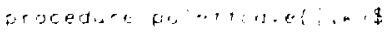

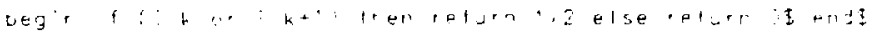

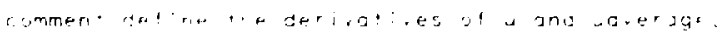

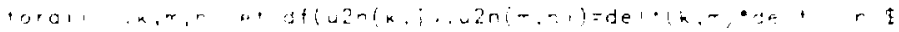

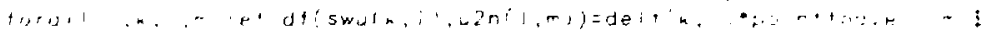

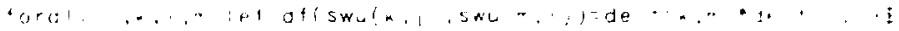

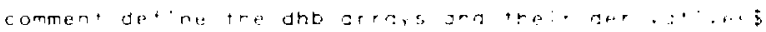

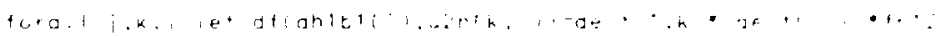

$-\mathrm{de}+1,-\cdots,+1,1, \cdots, \$$

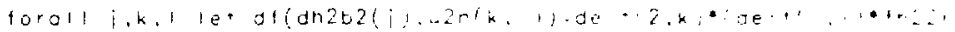

- de $1+i,-i, i-12, \quad 1,1, \$$

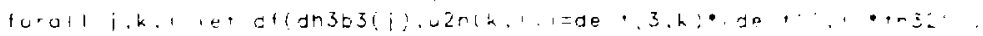

a

comment defire the bs ond. sq oervatives\$

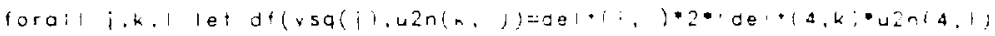
$+d e l f(5, k) \cdot u 2 n(5, i)+d e !+(6, k) \cdot u \geq n(6,1)) \$$

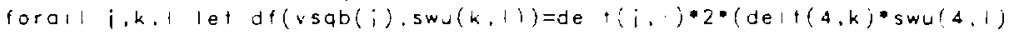
+de $(1(5, k) \bullet$ swu $(5,1)+d e 1+(6, k) *$ swuit, 1$)) \$$

forall $i, k,:$ let $d f(v 5 g b(i), u 2 n(k, 1))=0^{\circ} n+10 a v e(i, 1)$

-df(vsqb(i), swu(k,i))\$ 
comment define the bsq operator;

foral i,k.t let $d f(\operatorname{bsq}(i), u 2 n(k, 1))=d e l+(i, 1) * 2 *(d e i t(1, k) * u 2 n(1,1)$ + del $+(2, k) \cdot u 2 n(2,1)+d e 11(3, k) \cdot u 2 n(3,1)) \$$

comment define the b2b1, etc. derivotives

where $b 2 b 1=b 2 b(i) *(f h 22(i) \cdot u 2 n(2, i)-f h 22(i-1) * u 2 n(i-1)) * h 12 i(i)$ :

foral, i.k.l let dt(b2bi(i),u2n(k, l))=h12bi(i)*(dh2b2(i)*

$d \uparrow(b 2 b(i), u 2 n(k, i))+b 2 b(i) \cdot d f(a h 2 b 2(i ;, u 2 n(k, i))) \$$

fora $i, k, 1$ let $d f(b 3 b 1(i), J 2 n(k, 1))=h 13 b i i j) \cdot(\operatorname{dn} 3 b 3(i) *$

$d+(b 3 b(i j . .2 n(k .1))+b 3 b(i) \cdot d f(\operatorname{dn} 3 b 3(i), u 2 n(k, 1))) \$$

commenl detire the derivatives of eto and etab\$

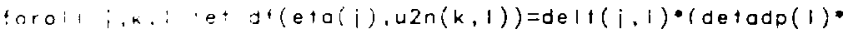

dfipressl i, $u 2 n(k, 1))+d e t+(k, 7) *$ detadr(lj) $\$$

loral $i, k, 1$ le df(etab(i), swu(k, l))=delt $(i, 1) *(\operatorname{detado}(i) *$

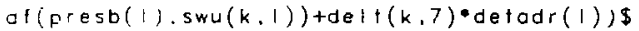

forai i,k.l let df(etab(i),u2n(k, l))=pointtoove(i, 1$)$

- $d f(e+a b(i), s w u(k, j)) \$$

commen' define the ortifical viscasity operators $q 9, q 2, q 3$ :

iora! i let $\left.q^{\prime}(i)=c z\right) * \operatorname{rob}(i j *(u 2 n(4, i)-u 2 n(4, i-1)) \cdots 2 \$$

foral i let $q 2(i)=c z 2 * \operatorname{rhot}(i) *(u 2 n(5, i)-u 2 n(5, i-1)) * 2 \$$

foral i tet a3(i)=cz3*rhob(i)*(u2n(6,i)-u2n(6,i-1))*2\$

comment define the derivotives of the pseudo-constant operators:

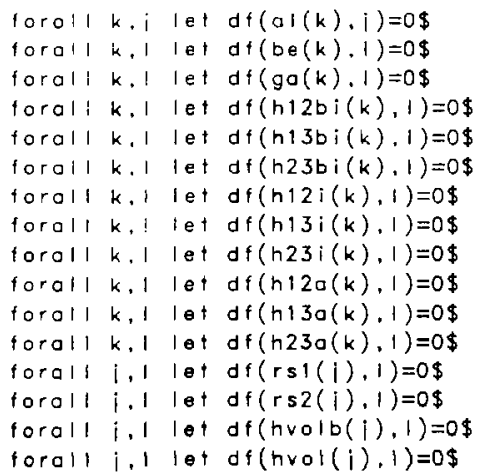




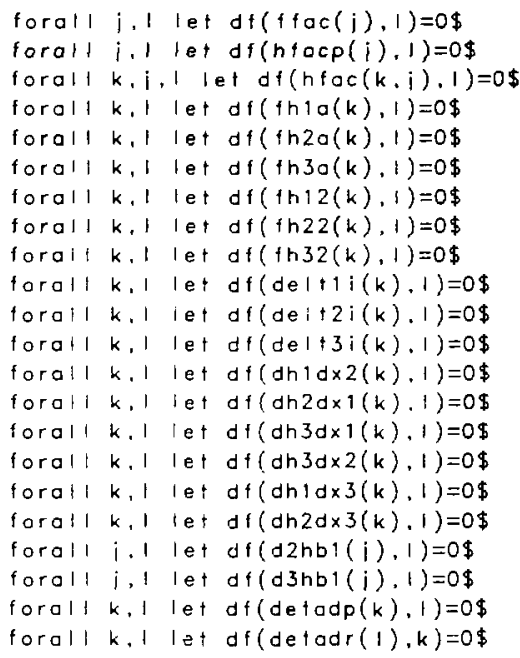

write "end definition of mhd ecations"

on rat, nat; order al, reyni, etab. swu:

orray symi(8,8). asymi $(8,8)$. symip $(8,8), \operatorname{asymip}(8,8)$;

comment procedure to write to disk:

procedure wrts();

begin

for $k:=1: 8$ do for $1:=1: 8$ do write symi $(1, k):=s y m i(i, k)$;

for $k:=1: 8$ do for $1:=1: 8$ do write symip(l,k):=symip(i,k):

for $k:=1: 8$ do for $1:=1: 8$ do write asymi(l,k):=asymi( , k ):

for $k:=1: 8$ do for $1:=1: 8$ do write asymipí, $k):=a s \forall m i p(1, k):$

end:

operator symm, asymm:

write "begin forming the symmetric \& antisym coefficients":

comment first we get the force and coriolus terms:

for al $m$ let $\operatorname{symm}(m)=f$ focp*force $(m, i+1)+d+b y 2 * c o r+1(m, i+1) / 2$;

comment then the flux terms;

for all $m$ let asymm $(m)=a I(m) * f \times(m, i+1)$; 


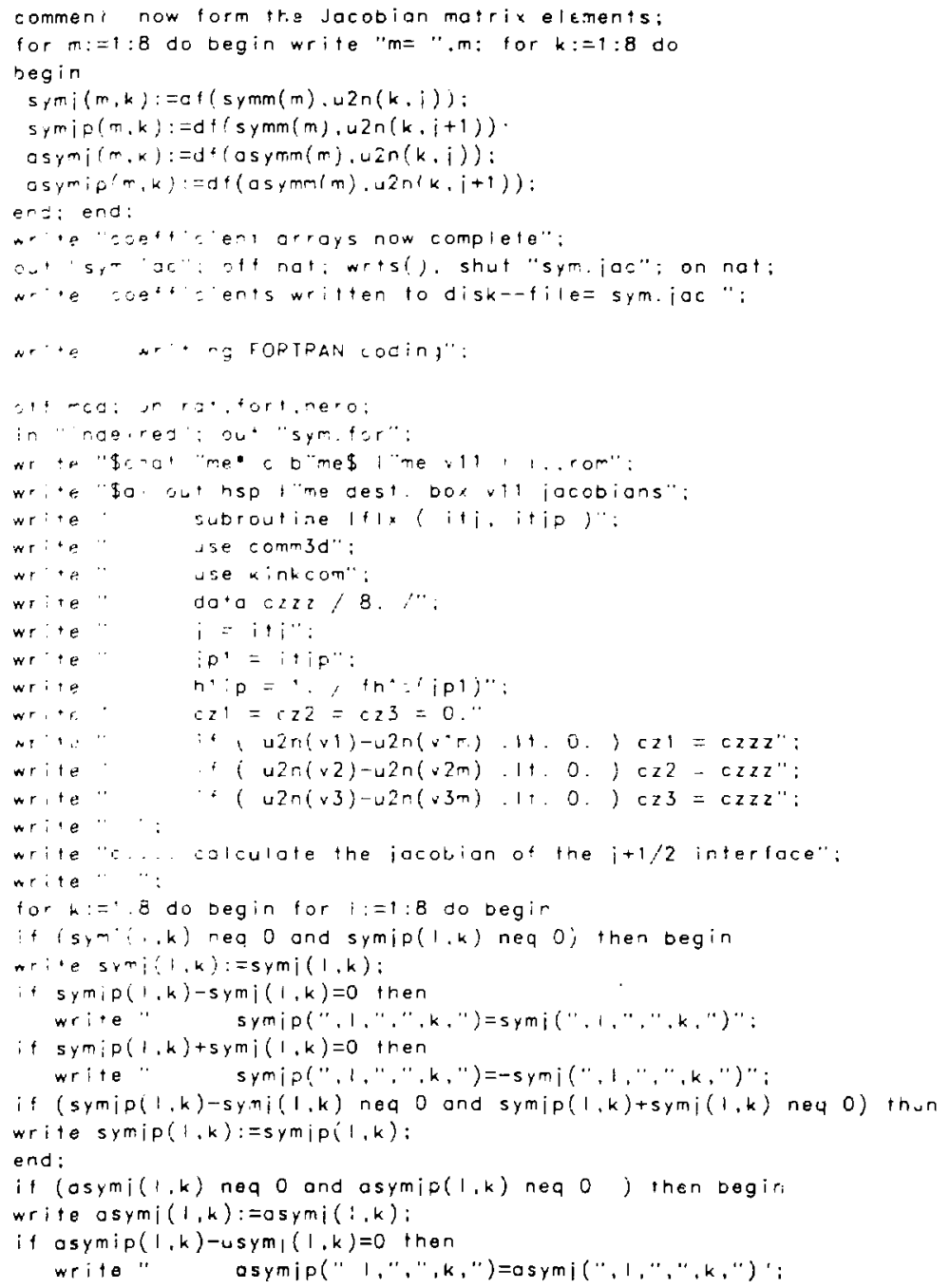




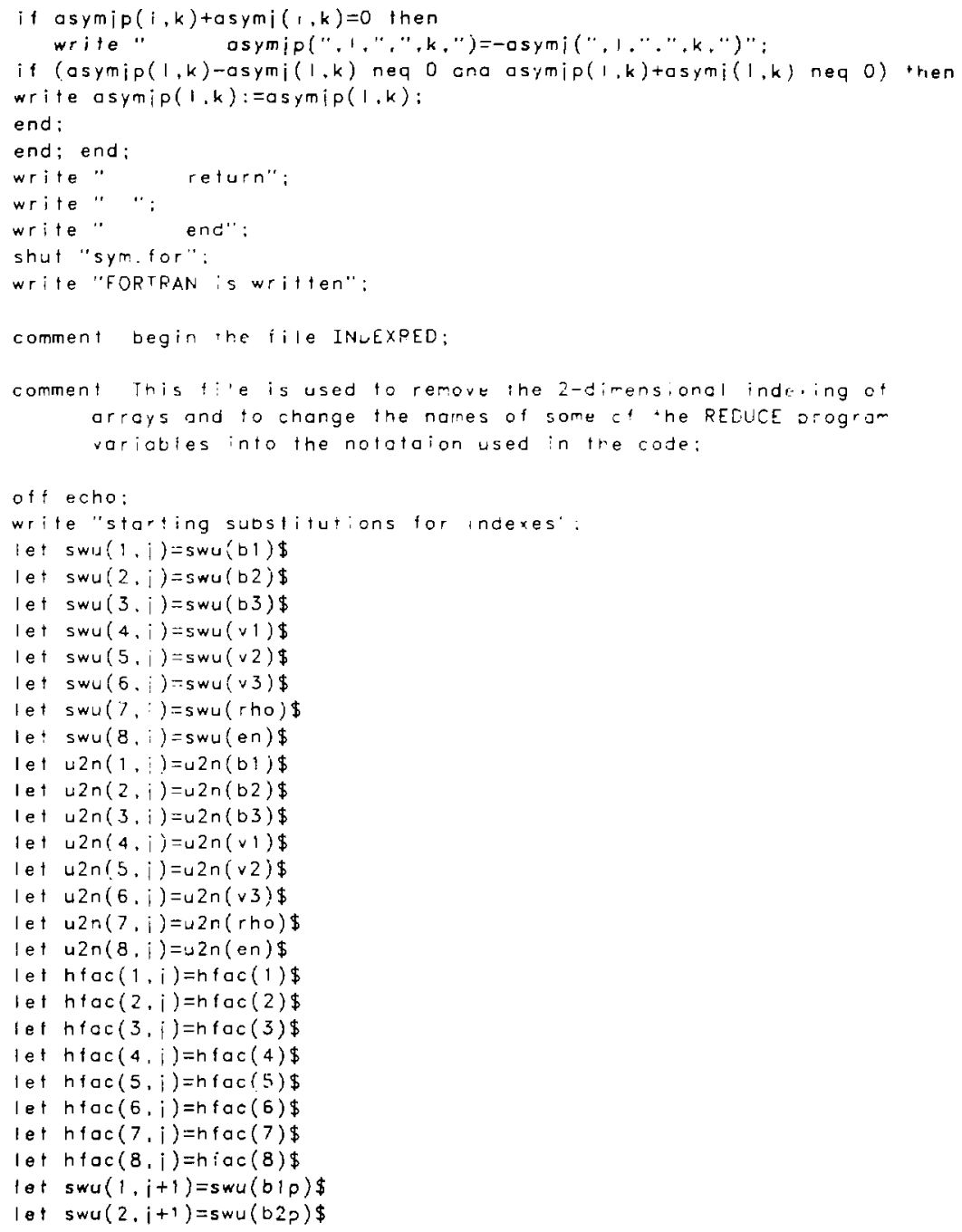




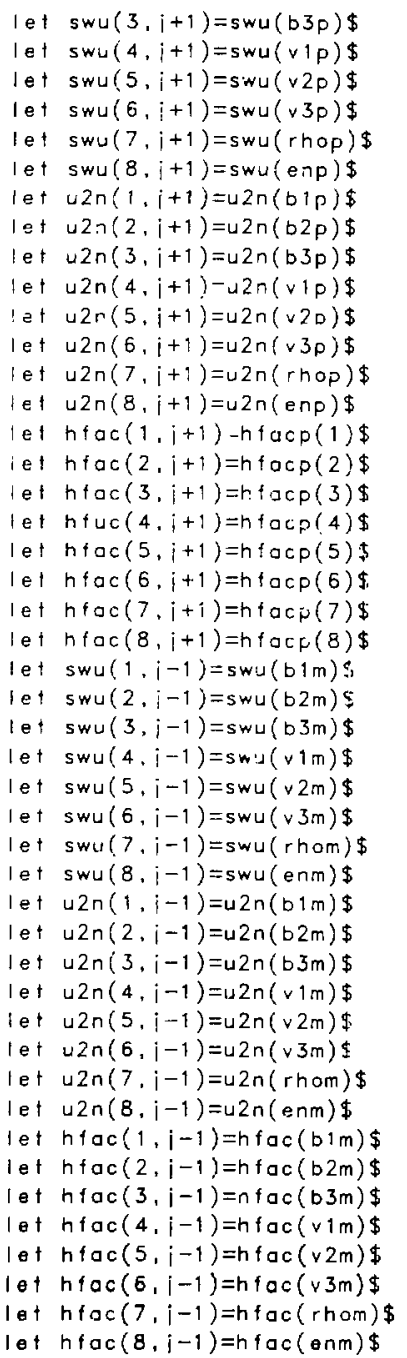




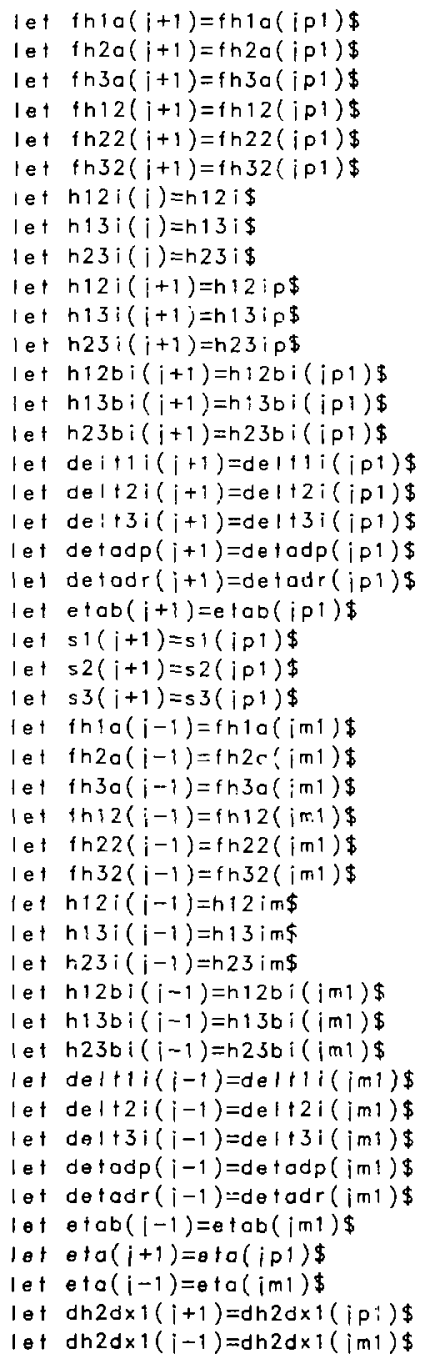




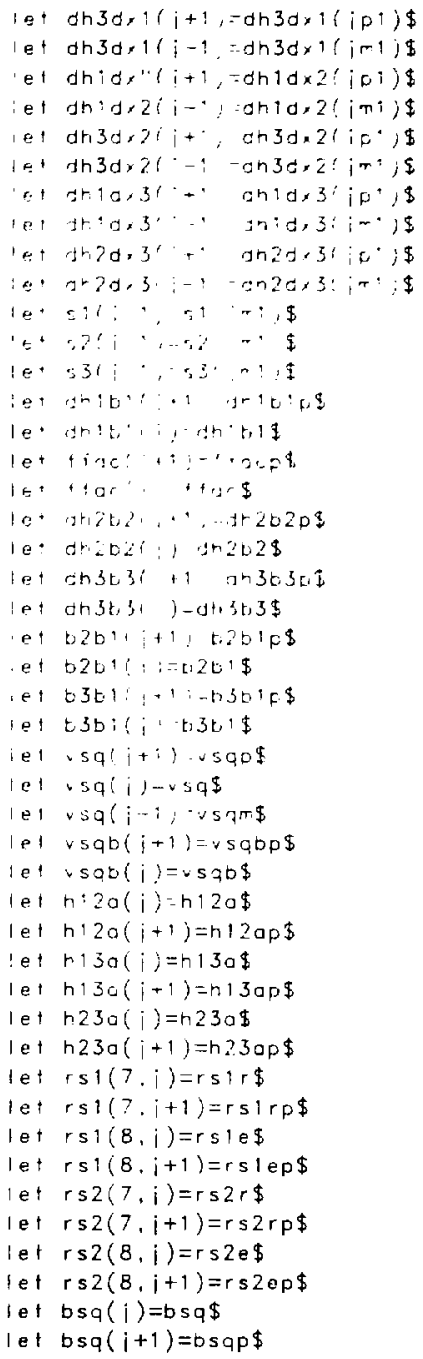




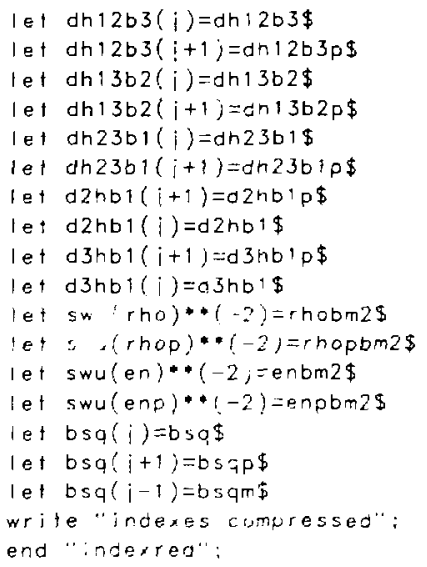

